\title{
Methods for Analysis of Cancer Chemopreventive Agents in Human Serum
}

Jeanice Brown Thomas and Katherine E. Sharpless, Editors
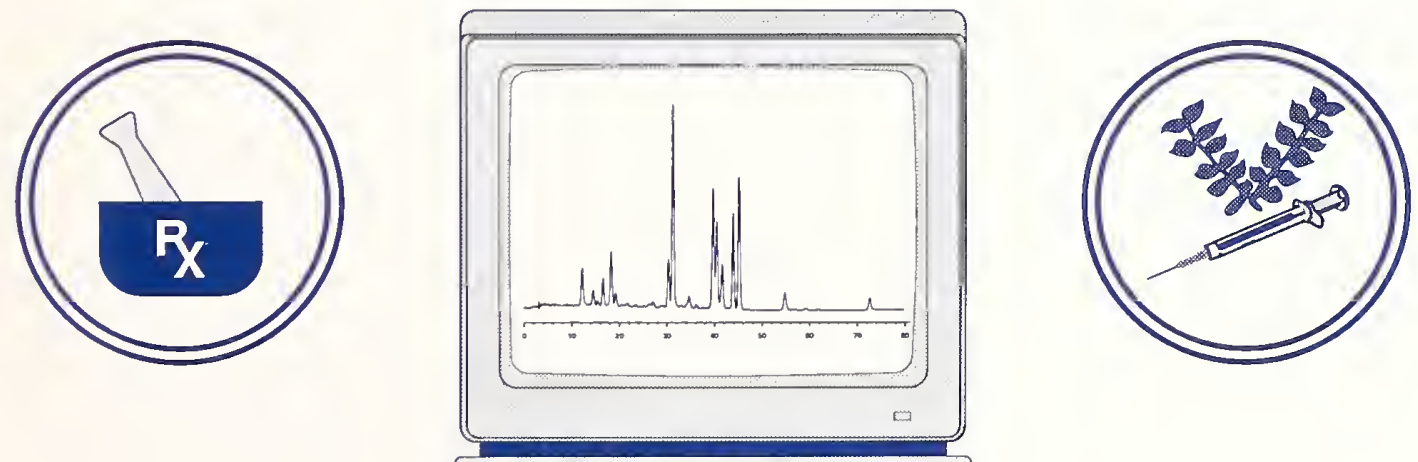

QC
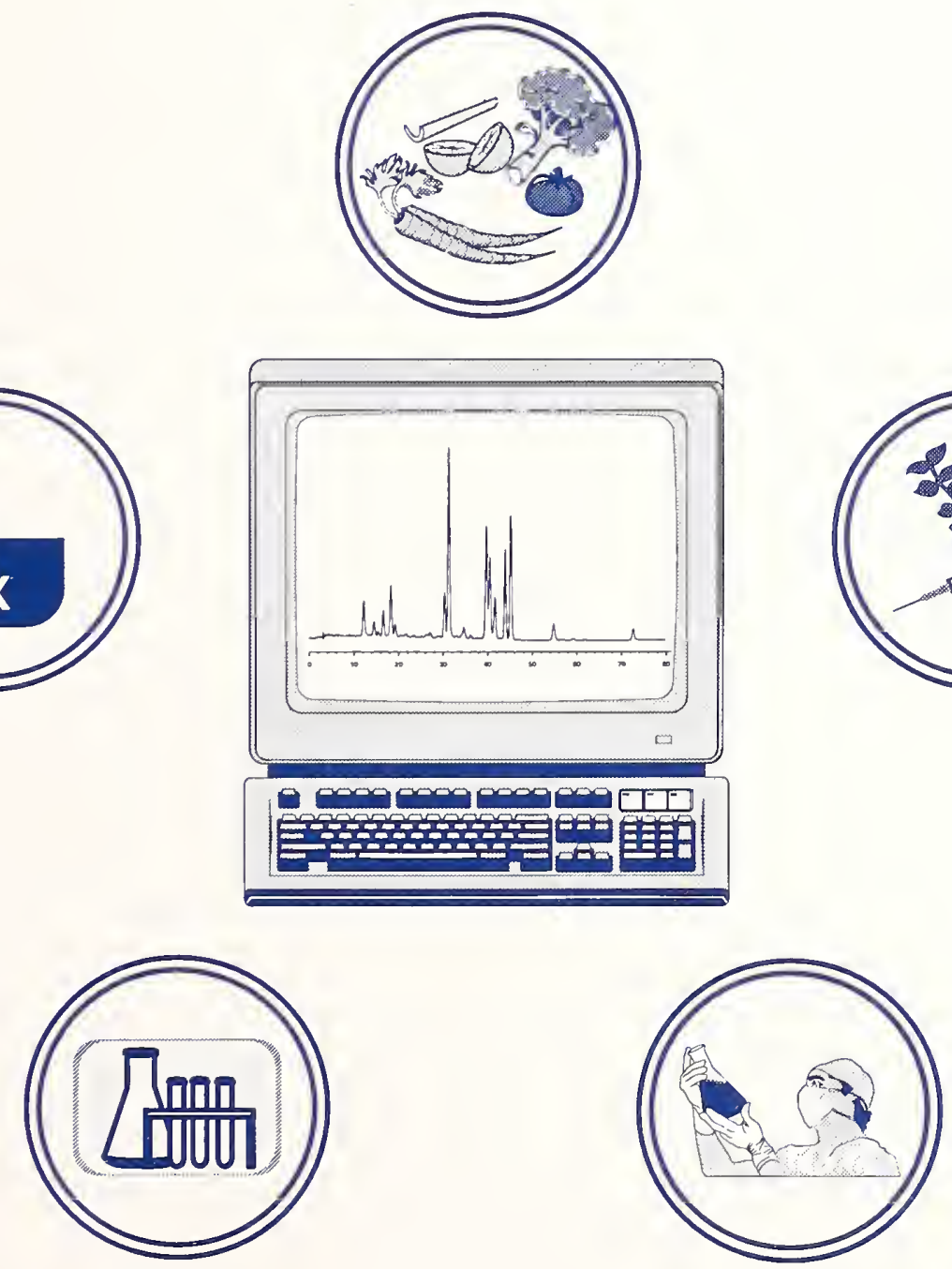

in the development of technology ... needed to improve product quality, to modernize manufacturing processes, to ensure product reliability ... and to facilitate rapid commercialization . . . of products based on new scientific discoveries."

NIST, originally founded as the National Bureau of Standards in 1901, works to strengthen U.S. industry's competitiveness; advance science and engineering; and improve public health, safety, and the environment. One of the agency's basic functions is to develop, maintain, and retain custody of the national standards of measurement, and provide the means and methods for comparing standards used in science, engineering, manufacturing, commerce, industry, and education with the standards adopted or recognized by the Federal Government.

As an agency of the U.S. Commerce Department's Technology Administration, NIST conducts basic and applied research in the physical sciences and engineering, and develops measurement techniques, test methods, standards, and related services. The Institute does generic and precompetitive work on new and advanced technologies. NIST's research facilities are located at Gaithersburg, MD 20899, and at Boulder, CO 80303. Major technical operating units and their principal activities are listed below. For more information contact the Public Inquiries Desk, 301-975-3058.

Office of the Director

- Advanced Technology Program

- Quality Programs

- International and Academic Affairs

Technology Services

- Manufacturing Extension Partnership

- Standards Services

- Technology Commercialization

- Measurement Services

- Technology Evaluation and Assessment

- Information Services

\section{Materials Science and Engineering} Laboratory

- Intelligent Processing of Materials

- Ceramics

- Materials Reliability

- Polymers

- Metallurgy

- Reactor Radiation

\section{Chemical Science and Technology}

\section{Laboratory}

- Biotechnology

- Chemical Kinetics and Thermodynamics

- Analytical Chemical Research

- Process Measurements ${ }^{2}$

- Surface and Microanalysis Science

- Thermophysics ${ }^{2}$

\section{Physics Laboratory}

- Electron and Optical Physics

- Atomic Physics

- Molecular Physics

- Radiometric Physics

- Quantum Metrology

- Ionizing Radiation

- Time and Frequency ${ }^{1}$

- Quantum Physics ${ }^{1}$
Manufacturing Engineering Laboratory

- Precision Engineering

- Automated Production Technology

- Intelligent Systems

- Manufacturing Systems Integration

- Fabrication Technology

Electronics and Electrical Engineering Laboratory

- Microelectronics

- Law Enforcement Standards

- Electricity

- Semiconductor Electronics

- Electromagnetic Fields ${ }^{1}$

- Electromagnetic Technology

- Optoelectronics ${ }^{1}$

Building and Fire Research Laboratory

- Structures

- Building Materials

- Building Environment

- Fire Safety

- Fire Science

\section{Computer Systems Laboratory}

- Office of Enterprise Integration

- Information Systems Engineering

- Systems and Software Technology

- Computer Security

- Systems and Network Architecture

- Advanced Systems

Computing and Applied Mathematics Laboratory

- Applied and Computational Mathematics ${ }^{2}$

- Statistical Engineering ${ }^{2}$

- Scientific Computing Environments ${ }^{2}$

- Computer Services

- Computer Systems and Communications ${ }^{2}$

- Information Systems

${ }^{1}$ At Boulder, CO 80303.

${ }^{2}$ Some elements at Boulder, CO 80303. 


\section{NIST Special Publication 874}

\section{Methods for Analysis of Cancer Chemopreventive Agents in Human Serum}

Editors

Jeanice Brown Thomas

and Katherine E. Sharpless

Chemical Science and Technology Laboratory

National Institute of Standards and Technology

Gaithersburg, MD 20899-0001

Research supported in part by:

Division of Cancer Prevention and Control

National Institutes of Health

National Cancer Institute

Bethesda, MD 20892

February 1995

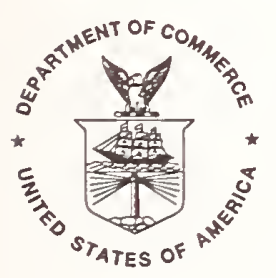

U.S. Department of Commerce

Ronald H. Brown, Secretary

Technology Administration

Mary L. Good, Under Secretary for Technology

National Institute of Standards and Technology

Arati Prabhakar, Director
Project Officer:

Dr. James A. Crowell

Chemoprevention Branch

Division of Cancer Prevention and Control

National Cancer Institute

Bethesda, MD 20892 
National Institute of Standards and Technology

Special Publication 874

Natl. Inst. Stand. Technol.

Spec. Publ. 874

128 pages (Feb. 1995)

CODEN: NSPUE2
U.S. Government Printing Office Washington: 1995
For sale by the Superintendent of Documents

U.S. Government Printing Office Washington, DC 20402-9325 


\section{ACKNOWLEDGMENTS}

We thank Ms. Donna Sirk for assistance in the preparation of this manual.

The following authors contributed papers:

Jeanice Brown Thomas, National Institute of Standards and Technology Richard G. Christensen, National Institute of Standards and Technology Neal E. Craft, Craft Technologies, Inc.

Thomas P. Davis, University of Arizona Health Sciences Center William A. MacCrehan, National Institute of Standards and Technology Winfred Malone, National Cancer Institute Sam A. Margolis, National Institute of Standards and Technology Willie E. May, National Institute of Standards and Technology Robert C. Paule, National Institute of Standards and Technology Herbert F. Pierson, Preventive Nutrition Consultants, Inc.

Roland Rieger, University of Ulm

Lane C. Sander, National Institute of Standards and Technology

Emil Schönberger, Hebrew University

Katherine E. Sharpless, National Institute of Standards and Technology Joseph H. Soares, Jr., University of Maryland Stephen A. Wise, National Institute of Standards and Technology Regina Ziegler, National Cancer Institute 



\section{INTRODUCTION}

The Micronutrients Measurement Quality Assurance (QA) Program was organized in 1984 by the National Cancer Institute (NCI) and the National Institute of Standards and Technology (NIST, formerly the National Bureau of Standards) to support measurement technology for selected fat- and water-soluble vitamins, carotenoids, zinc, and selenium in serum and plasma. This program was initiated as part of investigations supported by the NCI Division of Cancer Prevention and Control to study the possible role of these analytes as cancer chemoprevention agents.

Two of the main components of the program have been (1) methods development and validation, and (2) interlaboratory quality assurance through use of proficiency testing. Serum-based samples with assigned values for the target analytes and performance-evaluation standards are distributed by NIST to the laboratories for analysis. NIST staff provide the laboratories with technical feedback concerning their performance and suggestions for methods development and refinement. The results from the proficiency testing are used to establish a laboratory performance database for use by NCI to evaluate the quality of the analytical data from which epidemiological conclusions are to be made.

This report has been compiled as a result of NIST efforts to provide consultation in methods development and analytical-measurement troubleshooting to laboratories that measure vitamins $\mathrm{A}$ and $\mathrm{E}$, carotenoids, ascorbic acid, 4-hydroxyphenylretinamide, oltipraz, vitamin $\mathrm{K}_{1}$, and glycyrrhetinic acid in serum and plasma. A summary of factors that are essential to the method performance for each procedure is provided as a quick reference. The intended use of this summary is to help the practitioner focus on the steps needed to implement each procedure. The guidance offered within these pages should prove suitable for use in clinical laboratories and for NIST reference methods. 


\section{DISCLAIMER}

Certain commercial equipment, instruments, or materials are identified in this manual to specify adequately the experimental procedure. Such identification does not imply recommendation or endorsement by the National Cancer Institute or the National Institute of Standards and Technology, nor does it imply that the materials or equipment identified are necessarily the best available for the purpose. 


\section{TABLE OF CONTENTS}

Introduction $\ldots \ldots \ldots \ldots \ldots \ldots \ldots \ldots \ldots \ldots \ldots \ldots \ldots \ldots$ ii

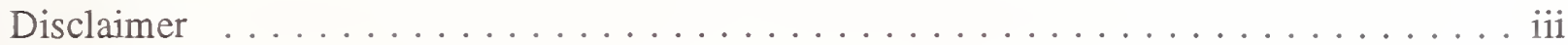

The purpose of this manual is to summarize several years of methods development and refinement for the measurement of selected fat- and water-soluble vitamins, carotenoids, oltipraz, and glycyrrhetinic acid in serum and plasma as part of the NISTNCI Micronutrients Measurement Quality Assurance Program. The manual is divided into six sections, each containing a series of publications pertaining to the measurement of the specified analyte(s). A summary of factors that are important to the method performance for each procedure is provided. This summary is included to help the analyst focus on the steps needed to implement each procedure.

Sections

1. Measurement of Fat-Soluble Vitamins and Carotenoids in Serum . . . . . . . . . 1-1

- Evaluation of Reversed-Phase Liquid Chromatographic Columns for Recovery and Selectivity of Selected Carotenoids . . . . . . . . . . . 1-3 K.S. Epler, L.C. Sander, R.G. Ziegler, S.A. Wise, and N.E. Craft

- Liquid Chromatographic Method for the Determination of Carotenoids, Retinoids, and Tocopherols in Human Serum

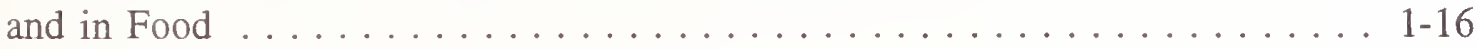
K.S. Epler, R.G. Ziegler, and N.E. Craft

- Determination of Retinol, $\alpha$-Tocopherol, and $\beta$-Carotene in Serum by Liquid Chromatography with Absorbance and Electrochemical Detection . . . . . . . . . . . . . . . . . . . . 1-28 W.A. MacCrehan and E. Schönberger

- Separation and Relative Distribution of All-trans- $\beta$-Carotene

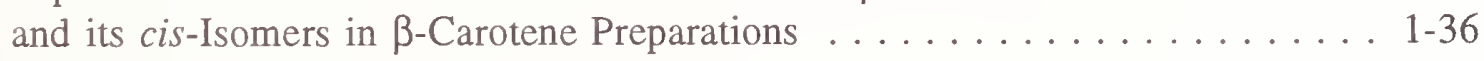
N.E. Craft, L.C. Sander, and H.F. Pierson

- Relative Solubility, Stability, and Absorptivity of Lutein and $\beta$-Carotene in Organic Solvents . . . . . . . . . . . . 1-49 N.E. Craft and J.H. Soares, Jr.

- Optimization of an Isocratic High-Performance Liquid Chromatographic Separation of Carotenoids . . . . . . . . . . . . 1-53 N.E. Craft, S.A. Wise, and J.H. Soares, Jr.

- Development of Engineered Stationary Phases for the

Separation of Carotenoid Isomers . . . . . . . . . . . . . . . . 1-59

L.C. Sander, K.E. Sharpless, N.E. Craft, and S.A. Wise

2. Determination of Glycyrrhetinic Acid ................... 2-1

- Determination of Glycyrrhetinic Acid in Human Plasma by High-Performance Liquid Chromatography . . . . . . . . . . . . . . 2-3

J. Brown Thomas, R.G. Christensen, R. Rieger, W. Malone, and W.E. May 
3. Measurement of Oltipraz in Serum . . . . . . . . . . . . . . . . . . . 3-1

- Determination of Oltipraz in Serum by High-Performance

Liquid Chromatography with Optical Absorbance and

Mass Spectrometric Detection ......................... 3-3

R.G. Christensen and W. Malone

4. Ascorbic and Dehydroascorbic Acids Analyses ... . . . . . . . . . . . . 4-1

- Ascorbic and Dehydroascorbic Acids Measured in Plasma Preserved

with Dithiothreitol or Metaphosphoric Acid . . . . . . . . . . . . . 4-3

S.A. Margolis, R.C. Paule, and R.G. Ziegler

- Stabilization of Ascorbic Acid in Human Plasma, and Its

Liquid-Chromatographic Measurement . . . . . . . . . . . . . . . . . . 4 4-9

S.A. Margolis and T.P. Davis

5. Determination of Vitamin $\mathrm{K}_{1}$ in Serum ............... 5-1

- Determination of Vitamin $\mathrm{K}_{1}$ in Serum ................. 5-3

W.A. MacCrehan and E. Schönberger

6. Measurement of (4-Hydroxyphenyl)retinamide in Serum . . . . . . . . . . . . 6-1

- NIST Measurements of (4-Hydroxyphenyl)retinamide in Serum . . . . . . . . 6-2

W.A. MacCrehan 


\section{LIST OF FIGURES}

Page

\section{Section 1: Measurement of Fat-Soluble Vitamins and Carotenoids in Serum}

Structures of Carotenoids Used in Test Mixture $\ldots \ldots \ldots \ldots \ldots \ldots \ldots \ldots$ 1-7

Separation of Test Mixture on a Monomeric $C_{18}$ Phase $\ldots \ldots \ldots \ldots \ldots$

Separation of Test Mixture on a Polymeric $C_{18}$ Phase . . . . . . . . . . . 1-9

Separation of Test Mixture on an Intermediate $C_{18}$ Phase $\ldots \ldots \ldots \ldots \ldots$

Structures of Retinol, trans- $\beta$-Apo-10'-Carotenal Oxime and the Carotenoids

Measured ....................................... 1-18

Structures of Retinyl Palmitate, Tocol, and $\alpha-, \gamma$, and $\delta$-Tocopherol . . . . . . . . 1-18

Chromatogram from the Reversed-Phase LC Separation of Retinol, trans- $\beta$-Apo-10'-Carotenal Oxime, Lutein, Zeaxanthin, $\beta$-Cryptoxanthin, trans-Lycopene, $\alpha$-Carotene, trans- $\beta$-Carotene, and Retinyl Palmitate in Human Serum Using Absorbance Detection. Chromatogram of Tocol, $\delta$-Tocopherol, $\gamma$-Tocopherol, and $\alpha$-Tocopherol Using Fluorescence Detection $\ldots . . . .1-21$

Chromatogram from the Reversed-Phase LC Separation of Retinol, Carotenoids, and Tocopherols in a Mixture of Apricots, Carrots, Creamed Corn, Creamed Peas, Green Beans, Orange Juice, Peaches, Spinach, Sweet Potatoes, Tomato Paste, and Winter Squash.

Fast-Isocratic Separation of the Micronutrients in a Serum Extract . . . . . . . . . . 1-31

Slow-Gradient-Elution Separation of Micronutrients in a Serum Extract . . . . . . . . 1-31

Hydrodynamic Voltammograms for All-trans-Retinol, Tocol, $\alpha$-Tocopherol, and All-trans- $\beta$-Carotene at a Glassy Carbon Electrode . . . . . . . . . . . . 1-32

Comparison of Absorbance and Electrochemical Detection of Carotenes . . . . . . 1-33

Comparison of the Absorbance with the Electrochemical Detection Results for the Determination of All-trans-Retinol, $\alpha$-Tocopherol, and $\beta$-Carotene . . . . . . . . . 1-34

Comparison of the Separation of an Algal $\beta$-Carotene Extract Using Reversed-Phase $\mathrm{C}_{18}$ LC Columns . . . . . . . . . . . . . . . . . . . . . . . . . 1-42 
List of Figures, Continued

Comparison of the Difference in the Separation Selectivity Using Methanol or Acetonitrile as the Mobile Phase . . . . . . . . . . . . . . . . . . . . 1-43

Representative Chromatograms of $\beta$-Carotene Preparations Separated Using a Wide-Pore, Polymeric $\mathrm{C}_{18}$ Column . . . . . . . . . . . . . . . . . . . . 1-44

Overlay of the Absorption Spectra of the Major $\beta$-Carotene Isomers Present

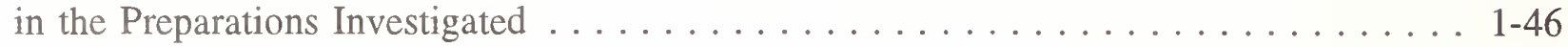

Percent Initial Absorbance at $\lambda_{\max }$ of Lutein and $\beta$-Carotene Monitored in 18 Organic Solvents Over a Period of 10 Days at Ambient Temperature . . . . . . . . . 1-52

The Effect of Nine Solvent Modifiers on the Separation of Seven Carotenoids . . . . . 1-55

The Effect of Three Concentrations of THF on the Separation of Seven

Carotenoids

The Effect of Five Column Temperatures on the Separation of Seven Carotenoids . . . 1-56

The Separation of Serum and Mixed Diet Carotenoids Using the Optimized

HPLC Method . . . . . . . . . . . . . . . . . . . . . . . . . . 1-56

Structures of Polar and Nonpolar Carotenoids . . . . . . . . . . . . . . . . 1-61

Column Selectivity Comparison for Various $\mathrm{C}_{18}$ Phases Prepared Using

Monomeric and Polymeric Syntheses . . . . . . . . . . . . . . . . . . 1-62

Effect of Endcapping for Polymeric $C_{18}$ Phases for Polar and Nonpolar Solutes ... . 1-63

Space Filling Models for $\beta$-Carotene and All-trans Hydrocarbon Chains . . . . . . . 1-64

Separations of Carotenoid Standards on Commercial and Monomeric $\mathrm{C}_{18}$

Columns and the Engineered $C_{30}$ "Carotenoid Column" .............. 1-65

Separation of Carotenoids from an Extract of SRM 2383, Vitamins and Carotenoids in Food . . . . . . . . . . . . . . . . . . . . . . . . . 1-66

Separation of a $\beta$-Carotene Preparation Containing a High Percentage of cis-Isomers on Commercial $\mathrm{C}_{18}$ Columns and "Carotenoid Column" . . . . . . . . . . . 1-66 
List of Figures, Continued

\section{Section 2: Determination of Glycyrrhetinic Acid}

Structures of $18 \beta$-Glycyrrhetinic Acid and the Acetate Ester of Glycyrrhetinic Acid. .

HPLC from the Analysis of a Standard Solution of $1 \mu \mathrm{g} / \mathrm{mL}$ GRA and

$3.4 \mu \mathrm{g} / \mathrm{mL}$ GRA Acetate Using Methanol-Water-Acetic Acid

HPLC from the Analysis of Plasma Extract Using Solid-Phase Extraction:

$0.38 \mu \mathrm{g} / \mathrm{mL}$ GRA and $2.9 \mu \mathrm{g} / \mathrm{mL}$ GRA Acetate . . . . . . . . . . . . . . 2-7

\section{Section 3: Measurement of Oltipraz in Serum}

Schematic of the On-line Extraction System . . . . . . . . . . . . . 3-5

Chromatograms of Serum Extracts . . . . . . . . . . . . . . . . . . 3-6

Single-Ion Monitoring of the $\mathrm{M}^{-}$Ion of Oltipraz from the Injection of a

Serum Extract Containing About 1 ng of Oltipraz . . . . . . . . . . . . . . . 3-7

Section 4: Ascorbic and Dehydroascorbic Acids Analyses

Chromatograms of Lyophilized and MPA-Preserved Human Plasma and AA-Supplemented Plasma With and Without DTT Treatment . . . . . . . . . . . . 4-5

Separation of Ascorbic Acid in Human Plasma on an HPX87H Column . . . . . . . 4-11

Separation of Ascorbic Acid on an Octadecylsilane Column . . . . . . . . . . . . 4 4-11

Concentration - Response Curves for Standard Solutions of Ascorbic Acid as a Function of Storage Time . . . . . . . . . . . . . . . . . . . . . 4-12

Effect of DTT and MPA on the Conversion of DHAA to Ascorbic Acid in Human Plasma . . . . . . . . . . . . . . . . . . . . . . . . . . . 4 4-13 
List of Figures, Continued

\section{Section 5: Determination of Vitamin $K_{1}$ in Serum}

The Catalytic Reduction of Quinone .................... 5-3

Block Diagram of Vitamin $\mathrm{K}_{1}$ Catalytic Reduction/LC-Fluorescence System . . . . . . 5-7

Chromatogram of Fractionated Serum Extract $\ldots \ldots \ldots \ldots \ldots \ldots \ldots$

Section 6: Measurement of (4-Hydroxyphenyl)retinamide in Serum

Structure of Retinamides ... . . . . . . . . . . . . . . . . . . . 6-2 
LIST OF TABLES

\section{Section 1: Measurement of Fat-Soluble Vitamins and Carotenoids in Serum}

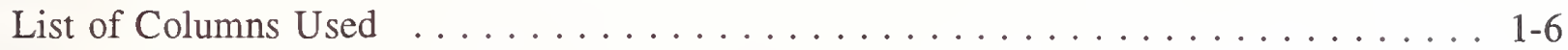

Resolution and Selectivity for Zeaxanthin/Lutein and $\beta$-Carotene/Lycopene on Selected $\mathrm{C}_{18}$ Columns Using Methanol and THF-Methanol Compared to the TBN/BaP $\alpha$ Value on Those Columns . . . . . . . . . . . . . . . . . . . . . . 1-9

Average Recovery of Carotenoids on All Columns Tested Using Different Mobile Phases . . . . . . . . . . . . . . . . . . . . . . . . . . . . . . 1-11

Columns Used in This Study Grouped According to Percent Recoveries . . . . . . . . 1-12

Average Percent Recovery for Polymeric, Intermediate, and Monomeric

Columns Tested . . . . . . . . . . . . . . . . . . . . . . . . . . . 1-12

Comparison of $k^{\prime}$ and Recovery for Columns from Different Lots . . . . . . . . . 1-13

Comparison of $k^{\prime}$ and Recoveries on Columns with Different Frit Materials . . . . . . 1-14

Absorptivities and Maximum Wavelengths Used for Calibration . . . . . . . . 1-18

Results of the Analysis of SRM 968a, Fat-Soluble Vitamins in Human Serum . . . . 1-23

Mean Standard Deviation and Relative Standard Deviation for Two Quality

Control Serum Samples Measured Fifteen Times Over a Five-Week Period . . . . . . . 1-24

Recovery of a Carotenoid Mixture on Selected Columns Before and After

Treatment with $0.05 \mathrm{M}$ Ammonium Acetate in Methanol With and Without the

Addition of $0.05 \%$ TEA to an Acetonitrile-Based Mobile Phase . . . . . . . . . 1-25

Linear Regression Calibration Curves for the Micronutrients by Each Detector . . . . . 1-33

Variation of Absorbance Signal for $\beta$-Carotene with Mobile Phase

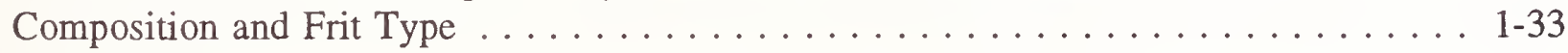

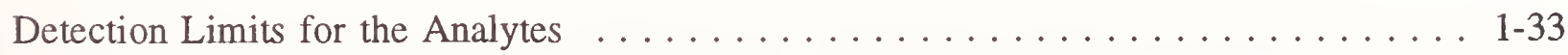

Determination of Micronutrients in a Human Serum Pool . . . . . . . . . . . . . . 1-34

Source and Description of $\beta$-Carotene Products Investigated $\ldots \ldots \ldots \ldots \ldots \ldots$ 1-38 
List of Tables, Continued

LC Column Characteristics . . . . . . . . . . . . . . . . . . . . . . . . 1-39

Spectrophotometric Concentration of $\beta$-Carotene Products Relative to the Gravimetric Concentrations . . . . . . . . . . . . . . . . . . . . . . . . . . 1-41

LC Determination of the Relative Composition of $\beta$-Carotene Isomers in $\beta$-Carotene Preparations . . . . . . . . . . . . . . . . . . . . . 1-45

List of Solvents, Sources, and Lot Numbers . . . . . . . . . . . . . 1-50

Relative Solubility and Absorptivity of Lutein and $\beta$-Carotene in

Organic Solvents . . . . . . . . . . . . . . . . . . . . . . . . 1-50

Relative Lutein and $\beta$-Carotene Degradation in Organic Solvents . . . . . . . . . 1-51

Bonded Phase Properties . . . . . . . . . . . . . . . . . . . . . .

Physical Properties of Alkyl-Modified Silica . . . . . . . . . . . . . . . . . 1-64

Physical Properties of Intermediate Pore Size Silicas . . . . . . . . . . . . . 1-64

\section{Section 2: Determination of Glycyrrhetinic Acid}

HPLC Measurements of $18 \beta$-Glycyrrhetinic Acid in Plasma After Ion-Pairing Solid-Phase Extraction . . . . . . . . . . . . . . . . . . . . 2-8

\section{Section 3: Measurement of Oltipraz in Serum}

Comparison of Oltipraz Concentrations Determined by Three Methods . . . . . . 3-7

\section{Section 4: Ascorbic and Dehydroascorbic Acids Analyses}

Summary of Analysis of Variance of Plasma Samples, Set 1, Supplemented with AA at High- and Low-Normal Concentrations . . . . . . . . . . . . . . . 4-5

Summary of Analyses of AA-Supplemented Plasma Samples, Set 2, Preserved in MPA, $50 \mathrm{~g} / \mathrm{L}$ 
List of Tables, Continued

Stability of Ascorbic and Dehydroascorbic Acids in MPA, $50 \mathrm{~g} / \mathrm{L}$, at $4{ }^{\circ} \mathrm{C} \ldots \ldots$. . 4-6

Stability of Ascorbic and Dehydroascorbic Acids in MPA, $50 \mathrm{~g} / \mathrm{L} \ldots \ldots . \ldots$. . . 6

Stability of Ascorbic Acid in Freeze-Dried and Frozen Plasma . . . . . . . . . . 4-12

Comparison of Round-Robin AA Values and Values Obtained by NBS (mg/L) . . . . 4-12

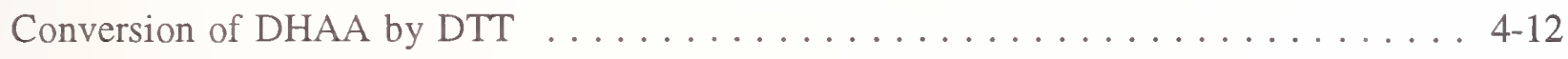

Ascorbic Acid Concentrations (mg/L) in Plasma of 11 Normal Subjects . . . . . . . 4 4-13 



\section{MEASUREMENT OF FAT-SOLUBLE VITAMINS AND CAROTENOIDS IN SERUM}

This section of the reference manual contains seven papers pertaining to the analysis of vitamins $A$ and $E$ and the carotenoids. Most of the essential factors listed below come from the first three papers (pages 1-3 through 1-35), which compare the recovery and selectivity of carotenoids on 65 reversed-phase columns and describe two liquid chromatographic methods employed by NIST for carotenoid and fat-soluble vitamin analyses. The paper beginning with page 1-36 compares $\beta$-carotene preparations available from several suppliers. An evaluation of various properties of lutein (a polar carotenoid) and $\beta$-carotene (a non-polar carotenoid) in different organic solvents is provided beginning with page 1-49. The separation of carotenoids using optimized parameters on a polymeric $C_{18}$ and a $C_{30}$ stationary phase developed at NIST are described in the last two papers (pages 1-53 through 1-66). Listed below are some of the more important factors related to these analyses.

\section{Important Factors}

\section{Calibration and Sample Preparation}

- Use a spectrophotometer and Beer's Law to calculate concentrations of calibration solutions. Correct the concentration for chromatographic purity at the wavelength at which you performed your Beer's Law calculation. We use the wavelength of maximum absorption.

- Because of its stability, we prefer $\beta$-apo-10'-carotenal oxime as an internal standard for the retinoids and carotenoids rather than $\beta$-apo- 8 '- or 10'-carotenal. Tocol is used as an internal standard for the tocopherols.

- To overcome the binding of carotenoids to lipoproteins in serum, the proteins must first be denatured with ethanol. Acetone, acetonitrile, methanol, and perchloric acid yield lower recoveries of the fat-soluble vitamins and carotenoids than ethanol.

- Use a water-immiscible organic solvent to extract the analytes from the serum matrix. Hexane has been most frequently used, with a large ratio of volume of extractant to serum.

- Use an antioxidant in the solvent used to reconstitute serum extracts, e.g., $30 \mu \mathrm{g} / \mathrm{mL} 2,6$ di-t-butyl-4-methylphenol (BHT). 
- In our cooled autosampler $\left(15{ }^{\circ} \mathrm{C}\right)$, significant losses of hydrocarbon carotenoids in serum extracts are observed in about 6 hours when extracts are reconstituted in ethanol. This is presumably a result of coprecipitation with lipids in the extract. The hydrocarbons (and lipids) may remain in solution longer when extracts are reconstituted in 50:50 ethyl acetate:ethanol.

- Since carotenoid concentrations are frequently near the detection limit, make sure that the serum extract is completely dissolved in the reconstituting solvent. Ultrasonic agitation is helpful.

\section{Chromatography}

- Use a $\mathrm{C}_{18}$ column to separate the fat-soluble vitamins and carotenoids in serum. Polymerically bonded $\mathrm{C}_{18}$ phases (and some intermediate phases) generally provide better selectivity for the separation of carotenoids than monomerically bonded $\mathrm{C}_{18}$ phases. The $\mathrm{C}_{30}$ column provides better separation of some carotenoid isomers than the $\mathrm{C}_{18}$ columns.

- Monomeric columns, on average, provide better recovery than polymeric columns.

- Methanol-based solvent systems provide higher carotenoid recoveries than acetonitrilebased systems.

- If you have to use an acetonitrile-based system, add ammonium acetate and triethylamine to improve carotenoid recoveries.

- To avoid the possible loss of carotenoids, eliminate stainless steel frits.

- Residual acids and metal activity on column packing materials cause carotenoid losses.

- Use Standard Reference Material 968b, Fat-Soluble Vitamins and Cholesterol in Human Serum, to validate your HPLC method.

For further information, please contact Katherine Sharpless at (301)975-3121 or Jeanice Brown Thomas at (301)975-3120. 


\title{
Evaluation of reversed-phase liquid chromatographic columns for recovery and selectivity of selected carotenoids
}

\author{
Katherine S. Epler and Lane C. Sander \\ Organic Analytical Research Division, Chemical Science and Technology Laboratory, National Institute of Standards and Technology, \\ Gaithersburg, MD 20899 (USA)
}

Regina G. Ziegler

Environmental Epidemiology Branch, Epidemiology and Biostatistics Program, Division of Cancer Etiology, National Cancer Institute, Bethesda, MD 20892 (USA)

\section{Stephen A. Wise and Neal E. Craft*}

Organic Analytical Research Division, Chemical Science and Technology Laboratory, National Institute of Standards and Technology, Gaithersburg, MD 20899 (USA)

(First received September 17th, 1991; revised manuscript received November 28th, 1991)

\begin{abstract}
Sixty commercially available and five experimental liquid chromatography columns were evaluated for the separation and recovery of seven carotenoid compounds. Methanol- and acetonitrile-based solvents (either straight or modified with ethyl acetate or tetrahydrofuran) were compared to determine which solvent systems and which columns provided better selectivity and recovery. Methanol-based solvents typically provided higher recoveries than did acetonitrile-based solvents. Polymeric $\mathrm{C}_{18}$ phases generally provided better selectivity for the difficult separation of lutein and zeaxanthin than did monomeric $\mathrm{C}_{18}$ phases.
\end{abstract}

\section{INTRODUCTION}

Epidemiologists have observed a lower incidence of lung cancer in people who have an above-average intake of fruits and vegetables [1,2]. Studies also suggest that the intake of fruits and vegetables may reduce the risk of cancers of the mouth, pharynx, larynx, esophagus, stomach, colon, rectum, bladder, and cervix [2]. Because low serum levels of $\beta$-carotene are associated with the subsequent development of lung cancer, $\beta$-carotene may be the protective factor present in the fruits and vegetables [2]. However, the lowered incidence of cancer may be due to other carotenoids that are co-ingested with the $\beta$-carotene, and serum levels of these other carotenoids have not been adequately studied. Liquid chromatography (LC) has been used to separate and measure $\beta$-carotene in serum [3] with concurrent measurement of $\alpha$-carotene [4-10] and sometimes lycopene [11-18]. In addtion to numerous cis/trans geometric isomers, human blood serum contains at least six structurally distinct carotenoids. To determine which carotenoid compound(s) may provide anti-cancer effects, it is important to separate and measure the major carotenoids in serum and in food. Consequently, a number of workers have reported the measurement of carotenoids other than lycopene, $\alpha$-, and $\beta$-carotene in serum 
[19-31], in skin [32], in human milk [33] and in foods [34 48].

The majority of carotenoid separations reported in the literature involve the use of reversed-phase LC, generally on a $\mathrm{C}_{18}$ stationary phase, although a few normal-phase LC separations have been reported $[21,24,35]$. Most workers using reversed-phase LC have used one of the following solvent systems: an acetonitrile-based eluent, an acetonitrile-based eluent to which ammonium acetate has been added, or a methanol-based eluent.

Acetonitrile-based eluents are used most frequently. In 1979, Zakaria et al. [49] separated lycopene, $\alpha$-carotene, and $\beta$-carotene in tomatoes on a $\mathrm{C}_{18}$ column using a mixture of chloroform and acetonitrile. In 1983, Nelis and De Leenheer [19] reported LC separations on $\mathrm{C}_{18}$ columns using acetonitrile or acetontrile and $8 \%$ methanol with various organic modifiers added [tetrahydrofuran (THF), diisopropyl ether, chloroform, dichloromethane, ethyl acetate]. Most workers have adapted one of these mixtures so that it provides acceptable results on their particular LC column $[4,5,8,9,11-13,16$ $18,20,22,25,26,30,31,35,38,40,46,48]$. Khachik and co-worker $[37,41,42,47]$ typically add hexane to a mixture of methanol, acetonitrile, and methylene chloride. Other workers have used acetonitrile modified with THF and water [36], with water and 2 -propanol $[44,45]$, or in a $1: 1$ mixture with ethanol [27].

The second basic method was described by Peng [6], and employs a mixture of acetonitrile, THF, methanol, and ammonium acetate. This method has been adapted by Nierenberg et al. [3,14,29], Kalman et al. [10], and Culling-Berglund et al. [32].

Methanol-based separations cited include the úse of straight methanol [15] or methanol that has been modified with THF or chloroform [35], with water and THF [7], with water and butanol [23], and with hexane [28].

Carotenoid separations reported in the literature employ a wide variety of $\mathrm{C}_{18}$ and other reversedphase columns from different manufacturers. Several workers have compared separations on a small number of commercially available columns: Nelis and De Leenheer [19] have described the effects of five organic modifiers on retention and selectivity of carotenoids on two different $\mathrm{C}_{18}$ columns. Bushway [35] has compared the selectivity of eight columns (two normal-phase and six reversed-phase) using several different solvent systems. Lauren and McNaughton [50] have compared ten reversedphase columns with respect to alfalfa carotenoids' elution order, retention time, peak height and shape, etc. using acetonitrile and ethyl acetate with or without $0.1 \%$ n-decanol.

Recovery of carotenoids from the LC column is an important factor in carotenoid analysis. Frequently, serum carotenoid concentrations are near the detection limit so maximum sensitivity is necessary for accurate carotenoid measurements. This can only be achieved if carotenoid recovery is essentially $100 \%$. Similarly, since epidemiological studies continue for extended periods, reproducibility is extremely important to the outcome of studies. Therefore only LC columns offering consistent and maximum recovery can yield the most meaningful results. Finally, there is a need to correlate carotenoid analysis by LC to older spectrophotometric measurements. If LC columns do not provide complete and consistent recovery of carotenoids, then LC measurements cannot be correlated with spectrophotometric measurements of carotenoids. Only one limited study has been reported that compared serum carotenoids measured by LC to total carotenoid concentration determined statically in a spectrophotometer [20]. We have compared sixty-five reversed-phase LC columns (primarily $\mathrm{C}_{18}$-modified silica) for selectivity and recovery of a mixture of seven carotenoids, six of which are present in the serum of most American populations. This is the first study to report absolute recovery of carotenoids from LC columns.

\section{EXPERIMENTAL ${ }^{a}$}

\section{Test mixture}

An ethanol solution containing approximately 2 to $10 \mathrm{mg} / \mathrm{l}$ each of lutein (Kemin Industries, Des Moines, IA, USA); zeaxanthin, $\beta$-cryptoxanthin (Atomergic Chemetals, Farmingdale, NY, USA); echinenone (Hoffmann-La Roche, Nutley, NJ,

\footnotetext{
a Certain commercial equipment, instruments, or materials are identified in this report to specify adequately the experimental procedure. Such identification does not imply recommendation or endorsement by the National Institute of Standards and Technology, nor does it imply that the materials or equipment identified are necessarily the best available for the purpose.
} 
USA); lycopene (extracted from tomato paste); and $x$ - and $\beta$-carotene (Sigma, St. Louis, MO, USA) was prepared, and $2-\mathrm{ml}$ aliquots of this solution were stored in amber glass vials at $-20{ }^{\circ} \mathrm{C}$. The respective concentrations of the compounds in the mixture were sufficiently different to provide some indication of identity based on chromatographic peak area. Prior to injection, $100 \mu \mathrm{l}$ THF were added to a vial of the carotenoid mixture, and the mixture was placed in an ultrasonic bath for $10 \mathrm{~min}$ to ensure dissolution. This solution was then transferred to a vial and placed in an autosampler where it was held at a constant temperature of $15^{\circ} \mathrm{C}$; the autosampler injected a $20-\mu$ l aliquot of the solution onto the column being tested.

\section{Chromatographic conditions}

A liquid chromatograph pumped the mobile phase at a rate of $1.5 \mathrm{ml} / \mathrm{min}$. Solvents were premixed so as not to depend on reproducible mixing by the pump; the solvents were sparged with helium. The column being tested was held at $27^{\circ} \mathrm{C}$ by the LC column oven.

\section{Columns}

A list of the columns tested, serial number, manufacturer, and classification of the bonded $\mathrm{C}_{18}$ stationary phase (polymeric, monomeric, or intermediate [51]) is provided in Table I. Reversed-phase columns that were potentially useful for the separation of carotenoids were donated by LC column manufacturers. Columns prepared from different production lots from the same manufacturer were also requested in order to assess reproducibility. Several columns that were currently in use in our laboratory were tested as well. Most of the columns tested were $\mathrm{C}_{18}, 250 \times 4.6 \mathrm{~mm}$ I.D., with $5-\mu \mathrm{m}$ particles. All columns were made of stainless steel. All columns were alkyl-silane modified silica, except for the Biotage Unisphere Polybutadiene, which was modified alumina. The polymeric/monomeric/ intermediate classification given in Table I refers to the type of stationary phase modification procedure, and is described in detail in the Results and Discussion section, as is the separation factor $\left(\alpha_{\text {TBN-BaP }}\right.$ value $)$ to which it is related.

Eluents based in methanol and acetonitrile were used in this study. Butylated hydroxytoluene-stabilized THF and ethyl acetate modifiers were added to the mobile phase for those columns that failed to elute all seven compounds with a capacity factor $\left(k^{\prime}\right) \leqslant 11$. Both THF $[3,6,7,10,11,14,22,29,32,35$, $36,43]$ and ethyl acetate $[19,50]$ have been used for carotenoid separations and represent two different solvent selectivity groups, III and VI, respectively. (Chlorinated solvents such as methylene chloride and chloroform were not tested because of a previous report of carotenoid losses caused by the hydrochloric acid that may be a trace contaminant in these solvents [52].) Prior to performing a separation, each column was flushed with THF at $1.5 \mathrm{ml} /$ min for 4 min to remove any compounds remaining on the column from a previous run. The column was then equilibrated at $1.5 \mathrm{ml} / \mathrm{min}$ for $15 \mathrm{~min}$ with a given eluent. In cases where $100 \%$ methanol or acetonitrile eluted the compounds from the column with a $k^{\prime}<7$, no attempt was made to increase retention by the addition of water because of the minimal solubility of carotenoids in water and the opportunity for on-column precipitation [19,23].

\section{Recovery determination}

The photodiode array detector (Model 990, Waters, Milford, MA, USA) used to monitor the LC effluent acquired absorbance spectra from 350 to $500 \mathrm{~nm}$ with $20 \mathrm{scans} / \mathrm{s}$ at 2 -s intervals. Data were acquired for $25 \mathrm{~min}$ at 0.5 a.u.f.s. Spectral data were used for peak identification when necessary. Visible absorbance at $450 \mathrm{~nm}$ was recorded by the data system for the quantitative comparison discussed below.

For the recovery study, the column was replaced with a 195-cm length of PTFE tubing (0.8-mm I.D.) to provide "peak" dispersion similar to the LC column. Using ethanol as the mobile phase, a 20- $\mu$ l injection of the test mixture was considered to have a peak area that represented $100 \%$ recovery. Five such replicate measurements were made at the beginning and at the end of each day's work. With a column in place, the individual areas of the integrated peaks were totalled, and the total area was normalized to the average area of the 10 replicate injections when no column was used. Because the test mixture contained small quantities ( 1 to $7 \%$ ) of impurities that certain columns may have been capable of resolving, all peaks eluting from a column were measured, not just the seven main peaks.

The linearity of the detector response was verified 


\section{TABLE I}

\section{LIST OF COLUMNS USED}

Columns are $\mathrm{C}_{18}$ except where noted.

\begin{tabular}{|c|c|c|c|c|c|}
\hline \multicolumn{2}{|c|}{ Supplier } & \multirow{2}{*}{$\begin{array}{l}\text { Column name } \\
\text { Ultrasphere ODS }\end{array}$} & \multirow{2}{*}{$\begin{array}{l}\text { Serial No. } \\
\text { 9UE1898 }\end{array}$} & \multirow{2}{*}{$\frac{x_{\mathrm{TBN} / \mathrm{BaP}}^{a}}{1.92}$} & \multirow{2}{*}{$\frac{\text { Classification }^{b}}{\text { Monomeric }}$} \\
\hline 1 & Beckman & & & & \\
\hline 2 & Beckman & Ultrasphere ODS & $8 \mathrm{UE} 2757$ & 2.01 & Monomeric \\
\hline 3 & Beckman & Ultrasphere ODS DABS & 7UE2306 & 2.00 & Monomeric \\
\hline 4 & Analytichem & Sepralyte $\mathrm{C} 18$ & $072851-18$ & 1.82 & Monomeric \\
\hline 5 & Separations Group & Vydac 218TP & $890130-16$ & 0.83 & Polymeric \\
\hline 6 & Separations Group & Vydac $201 \mathrm{TP}$ & $890130-23$ & 0.78 & Polymeric \\
\hline 7 & Separations Group & Vydac 201TP & $890131-26$ & 0.80 & Polymeric \\
\hline 8 & Biotage & Unisphere-PBD ${ }^{c}$ & 2167 & 3.06 & Monomeric \\
\hline 9 & ES Industries & Gamma Bond C18 & $19589-57-17992$ & 1.86 & Monomeric \\
\hline 10 & ES Industries & Chromegabond $\mathrm{C} 22\left(\mathrm{C}_{22}\right)$ & $19589-4-58-17994$ & 1.83 & Monomeric \\
\hline 11 & ES Industries & Chromegabond $\mathrm{MCl} 8$ & $19589-4-57-17991$ & 1.73 & Monomeric \\
\hline 12 & Supelco & LC- 18 & $18744 \mathrm{~F}$ & 2.03 & Monomeric \\
\hline 13 & Supelco & LC-18 & $18745 \mathrm{~F}$ & 2.02 & Monomeric \\
\hline 14 & Supelco & LC318 & 110224 & 2.05 & Monomeric \\
\hline 15 & Supelco & LC318 & 110232 & 2.04 & Monomeric \\
\hline 16 & Supelco & LC-PAH & 81695 & 0.70 & Polymeric \\
\hline 17 & Analytichem & Sepralyte C18 & $071912-14$ & 1.92 & Monomeric \\
\hline 18 & Alltech & Adsorbosphere C18 & 08039GA & 2.01 & Monomeric \\
\hline 19 & EM Science & LiChrospher 100 RP-18 & 86554563 & 1.45 & Intermediate \\
\hline 20 & Serva & Octadecyl & 03118 & 1.84 & Monomeric \\
\hline 21 & Serva & Triacontyl $\left(\mathrm{C}_{30}\right)$ & $-^{d}$ & & \\
\hline 22 & $\mathrm{~J} \& \mathrm{cW}$ & Accusphere ODS & 9050825 & 2.07 & Monomeric \\
\hline 23 & $\mathrm{~J} \& \mathrm{~W}$ & Accusphere ODS & 9072526 & 1.96 & Monomeric \\
\hline 24 & MacMod & Zorbax RX C8 $\left(\mathrm{C}_{8}\right)$ & AU2642 & 2.33 & Monomeric \\
\hline 25 & Perkin Elmer & Pecosphere $\mathrm{C} 18$ & 1119 & 2.00 & Monomeric \\
\hline 26 & YMC & A 303 & 42511 & 1.97 & Monomeric \\
\hline 27 & YMC & AMP303 & 4259 & 2.01 & Monomeric \\
\hline 28 & YMC & AP303 & 4250 & 2.06 & Monomeric \\
\hline 29 & J.T. Baker & exper WP C18 $8^{e}$ & $1294-43$ & 0.57 & Polymeric \\
\hline 30 & J.T. Baker & exper WP C18 ${ }^{e}$ & $1294-26$ & 0.93 & Polymeric \\
\hline 31 & J.T. Baker & exper WP C18 & $1294-29$ & 0.73 & Polymeric \\
\hline 32 & J.T. Baker & exper WP C18 & $1239-64 \mathrm{C}$ & 1.42 & Intermediate \\
\hline 33 & J.T. Baker & exper WP C18 & $1239-64 \mathrm{D}$ & 0.22 & Polymeric \\
\hline 34 & ES industries & Chromegabond $\mathrm{PFP}^{f}$ & $19589-4-58-17993$ & 0.88 & Polymeric \\
\hline 35 & ES Industries & Chromegabond BF-C18 & $21389-4-60-18079$ & 1.04 & Intermediate \\
\hline 36 & J.T. Baker ${ }^{\theta}$ & Bakerbond $\mathrm{Cl} 8$ & A29113-01 & 1.25 & Intermediate \\
\hline 37 & J.T. Baker ${ }^{\theta}$ & Bakerbond WP C18 & B36097.25 & 0.51 & Polymeric \\
\hline 38 & J.T. Baker ${ }^{\theta}$ & Bakerbond WP C18 & none & 0.54 & Polymeric \\
\hline 39 & J.T. Baker ${ }^{g}$ & Bakerbond WP C18 & B33125.16 & 1.09 & Intermediate \\
\hline 40 & Keystone Sci. & ODS Hypersil & 03708 & 1.95 & Monomeric \\
\hline 41 & Shiseido & Capsell Pak C18 & SG120 & 1.99 & Monomeric \\
\hline 42 & Nacalai Tesque & Cosmosil 5C18-P Waters & $391-03$ & 2.04 & Monomeric \\
\hline 43 & Nacalai Tesque & Cosmosil 5C18 Waters & $390-47$ & 1.85 & Monomeric \\
\hline 44 & Waters & Nova-Pak C18 & Т93242 & 1.97 & Monomeric \\
\hline 45 & YMC & ASP $303-5$ & 42541 & 2.05 & Monomeric \\
\hline 46 & Hewlett-Packard & ODS Hypersil & $79926 \mathrm{OD}-584$ & 1.98 & Monomeric \\
\hline 47 & Hewlett-Packard & LiChrospher 100 RP-18 & 79925 OD -584 & 1.50 & Intermediate \\
\hline 48 & Brownlee & Spheri-5 ODS & 102454 & 1.26 & Intermediate \\
\hline 49 & Bio-Rad & Hi-Pore RP318 & 890215-11 No. 82 & 0.59 & Polymeric \\
\hline 50 & Macherey-Nagel & Nucleosil 5 PAH & $90702 \mathrm{~B}$ & 0.36 & Polymeric \\
\hline 51 & Rainin $^{\theta}$ & Microsorb C18 & 10681 & 1.78 & Monomeric \\
\hline 52 & Phase Separations & Spherisorb S5 PAH & $23 / 12300-1046$ & 0.82 & Polymeric \\
\hline 53 & Phase Separations & Spherisorb S5 ODS & $29 / 69 \quad 19-1372$ & 1.68 & Intermediate \\
\hline
\end{tabular}


TABLE I. (continued)

\begin{tabular}{|c|c|c|c|c|c|}
\hline \multicolumn{2}{|c|}{ Supplier } & \multirow{2}{*}{$\begin{array}{l}\text { Column name } \\
\text { Spherisorb S5 ODS }\end{array}$} & \multirow{2}{*}{$\begin{array}{l}\text { Serial No. } \\
\text { 30/35 06-10-F1 }\end{array}$} & \multirow{2}{*}{$\frac{\alpha_{\text {TBN/BaP }}{ }^{a}}{1.50}$} & \multirow{2}{*}{$\frac{\text { Classification }^{\circ}}{\text { Intermediate }}$} \\
\hline 54 & Phase Separations & & & & \\
\hline 55 & Carlo Erba & Erbasil 5 C18/L & 517250502 & 1.76 & Monomeric \\
\hline 56 & Carlo Erba & Erbasil $5 \mathrm{C} 18 / \mathrm{M}$ & 517250503 & 1.28 & Intermediate \\
\hline 57 & Carlo Erba ${ }^{h}$ & Erbasil $5 \mathrm{C} 18 / \mathrm{H}$ & 517250504 & 0.91 & Polymeric \\
\hline 58 & Brownlee & Spheri-5 RP-18 & 109083 & 1.92 & Monomeric \\
\hline 59 & MacMod & Zorbax RX C18 & 880967.902 & 1.50 & Intermediate \\
\hline 60 & Phenomenex & Ultracarb 5 ODS 20 & $\mathrm{PP} / 4953 \mathrm{C}$ & 1.95 & Monomeric \\
\hline 61 & Phenomenex & Ultracarb 5 ODS30 & $\mathrm{PP} / 4954 \mathrm{C}$ & 2.01 & Monomeric \\
\hline 62 & J.T. Baker & Bakerbond WP C18 & $1314-13$ & 0.89 & Polymeric \\
\hline 63 & J.T. Baker & Bakerbond WP C18 & DS097 & 0.67 & Polymeric \\
\hline 64 & Brownlee & Spheri-5 ODS & 102402 & 1.42 & Intermediate \\
\hline 65 & $\mathrm{MacMod}^{g}$ & Zorbax ODS & F36560 & 1.80 & Monomeric \\
\hline
\end{tabular}

- Relative retention of 1,2:3,4:5,6:7,8-tetrabenzonaphthalene (TBN) to benzo[a]pyrene (BaP); see Results and Discussion section or ref. 51.

- As determined by evaluation of SRM 869 (see refs. 51 and 53).

c $\mathrm{PBD}=$ polybutadiene.

- Returned to supplier before value could be determined.

- Experimental columns with varying surface coverage.

$s$ PFP $=$ pentafluorophenol.

- Used in laboratory prior to evaluation in this study.

" Not tested due to excessive backpressure (>200 bar).

by injecting varying amounts of the mixture of seven carotenoids. The detector response was linear from 50 to $300 \%$ of the amount injected for evaluation of the columns.

\section{RESULTS AND DISCUSSION}

Sixty-five reversed-phase LC columns (Table I) were evaluated to determine selectivity and recovery of selected carotenoids. Lutein, zeaxanthin, $\beta$-cryptoxanthin, echinenone, lycopene, and $\alpha$ - and $\beta$-carotene were selected for use in the test mixture, and are shown in Fig. 2. Excluding echinenone, which is present in the human populations that consume echinoderms (sea urchins, starfish), these compounds account for more than $90 \%$ of the carotenoids present in American serum [20]. Echinenone was included because it has intermediate polarity and has been used as an internal standard for carotenoid measurements [20].

\section{Column classification}

A system has been developed for the classification of $\mathrm{C}_{18}$ stationary phases based on their separation of a mixture of polycyclic aromatic hydrocarbons (PAHs) [51]. The application of this clas- sification scheme has proven useful for selecting columns for separating PAH isomers and steroids, two classes of compounds with rigid molecular

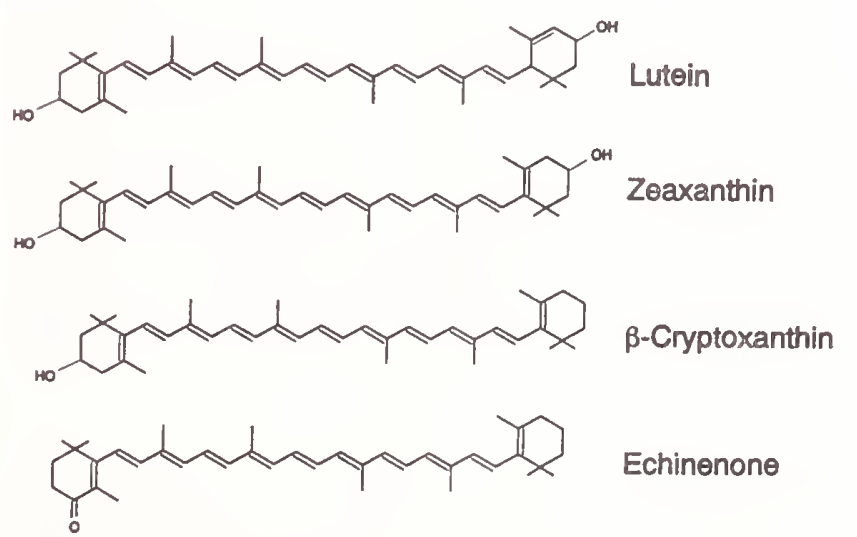

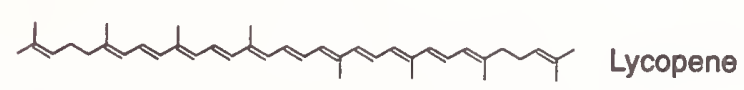

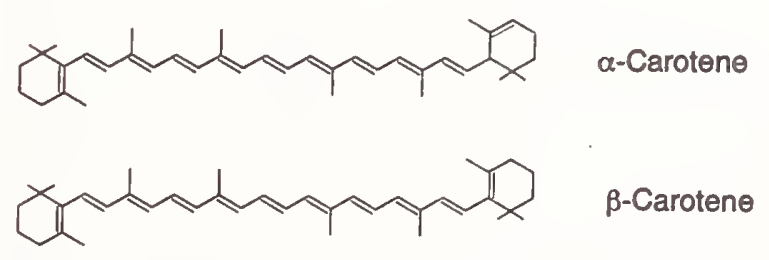

Fig. 1. Structures of carotenoids used in test mixture. 
structures [51]. Because carotenoid isomers also have a rigid structure, it was thought that the classification scheme might provide insight into retention mechanisms for carotenoids, as well as provide assistance in column selection.

The $\mathrm{C}_{18}$ columns in this study were classified into three stationary phase types using Standard Reference Material (SRM) 869, Column Selectivity Test Mixture for Liquid Chromatography [51,53]. The SRM is a mixture of three PAHs: benzo[a]pyrene (BaP), 1,2:3,4:5,6:7,8-tetrabenzonaphthalene (TBN), and phenanthro [3,4-c]phenanthrene. Columns were classified by calculating the relative retention of TBN to BaP. The resulting $\alpha_{\mathrm{TBN}-\mathrm{BaP}}$ values were grouped as follows: those columns with an $\alpha_{\mathrm{TBN}-\mathrm{BaP}}$ less than 1 are classified as polymeric, those with an $\alpha_{\mathrm{TBN}-\mathrm{BaP}}$ between 1 and 1.7 are classified as intermediate, and those with an $\alpha_{\mathrm{rBN}-\mathrm{BaP}}$ greater than 1.7 are classified as monomeric. The $\alpha_{\text {TBN-Bap }}$ values for each column are provided in Table I. These values may not be directly comparable for the five columns whose stationary phases were not $C_{18}$. Nonetheless, 35 of the columns tested were classified as monomeric, 17 were polymeric and 12 were intermediate at $27^{\circ} \mathrm{C}$.

This classification scheme is strongly related to the procedures used in the bonded-phase synthesis, i.e., monomeric phases prepared by the reaction of silica with monofunctional silanes (usually in the absence of water) have properties that differ from polymeric phases prepared by reaction of silica with trifunctional silanes in the presence of water. (The third group is an arbitrary classification for which phase chemistry is less certain; this group has properties that are intermediate to the monomeric and polymeric classes.)

\section{Selectivity}

Optimum carotenoid selectivity of each column was difficult to assess, since a mobile phase was not tailored for each. Our design was limited to an isocratic, nonaqueous binary mobile phase separation, with the capacity factor, $k^{\prime}$, manipulated through the addition of THF or ethyl acetate such that $7 \leqslant$ $k^{\prime} \leqslant 11$. Under the defined conditions, lutein and zeaxanthin were the most difficult carotenoids to resolve due to their structural similarity and early elution.

No monomeric $C_{18}$ column evaluated in this study was able to resolve lutein and zeaxanthin using methanol or methanol-based solvents. Using acetonitrile-based solvents, monomeric $\mathrm{C}_{18}$ columns were sometimes able to separate this pair partially. The polymeric $C_{18}$ columns were usually able to separate lutein and zeaxanthin. Typical chromatograms for columns classified as monomeric, polymeric, and intermediate are shown in Figs. 2, 3 and 4, respectively; chromatographic conditions are described in those figures. In Table II, resolution $(R)$ and $\alpha$ values for zeaxanthin/lutein and $\beta$-carotene/lycopene are shown for a representative onethird of the columns. The actual frequency and inability of a class of columns to separate lutein and zeaxanthin is represented, i.e., if one in every three monomeric columns failed to separate lutein and zeaxanthin, then one of every three numbers shown for the monomeric columns in Table II is for a column that failed to separate this pair. Using monomeric and most intermediate columns, the elution of lycopene was followed by that of $\alpha$ - and $\beta$-caro-

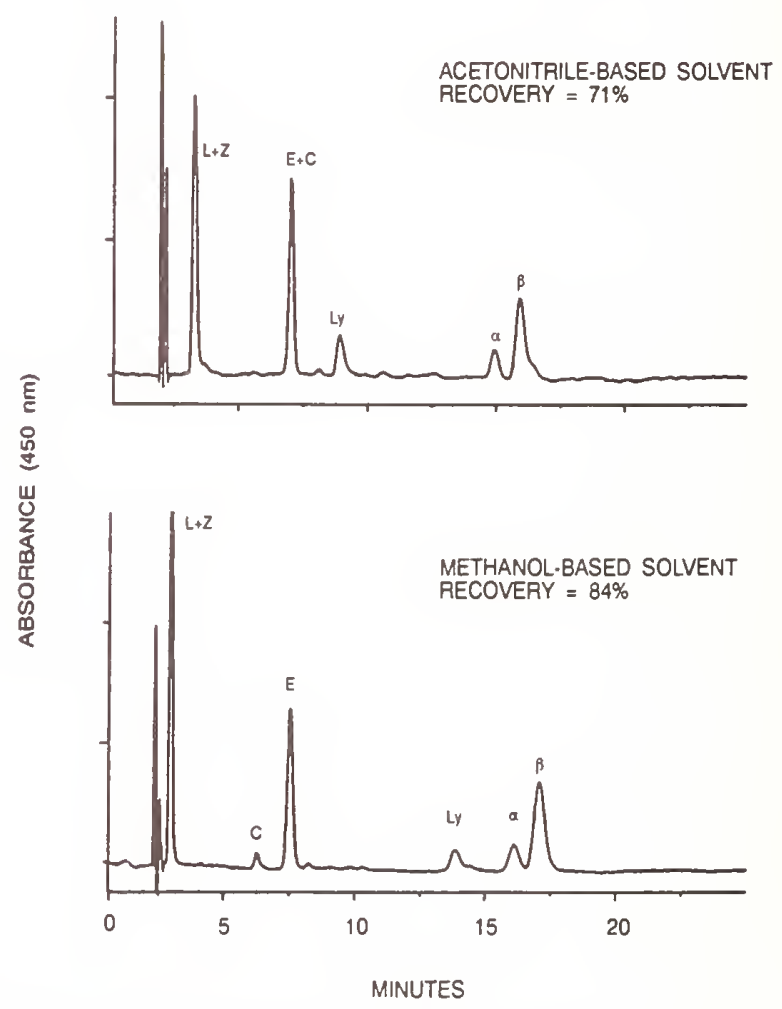

Fig. 2. Separation of test mixture on a monomeric $C_{18}$ phase. Eluent is THF-methanol (10:90) or THF-acetonitrile (15:85) at a flow-rate of $1.5 \mathrm{ml} / \mathrm{min}$. Legend: $\mathrm{L}=$ lutein, $\mathrm{Z}=$ zeaxanthin, $\mathrm{C}=\beta$-cryptoxanthin, $\mathrm{E}=$ echinenone, $\mathrm{Ly}=$ lycopene, $\alpha=$ $\alpha$-carotene, $\beta=\beta$-carotene. 


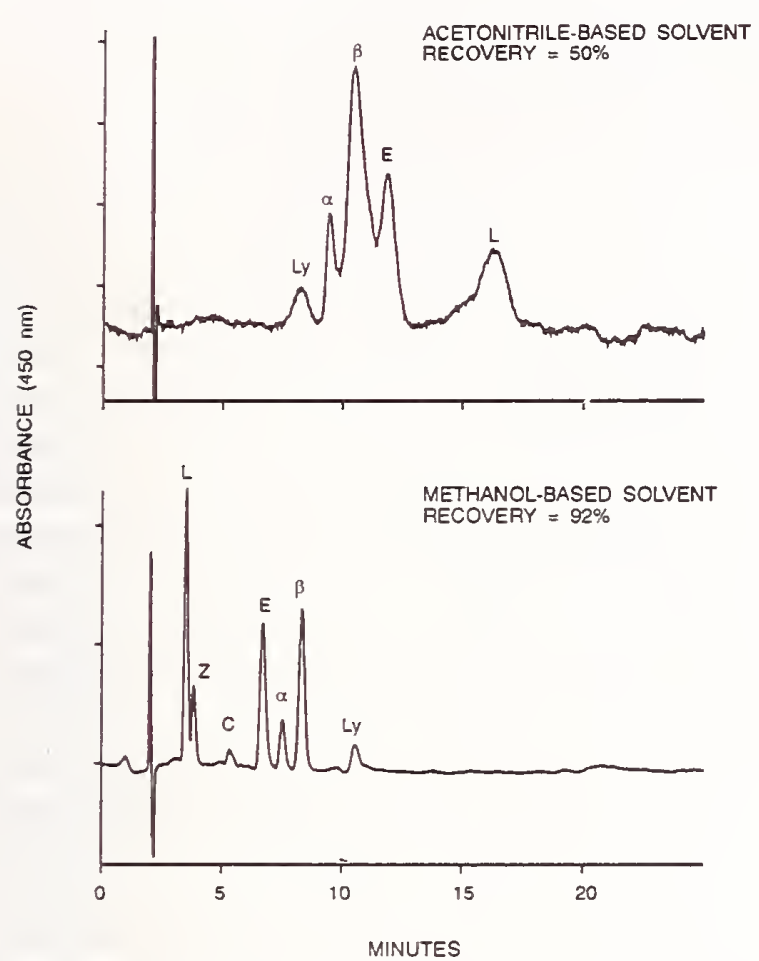

Fig. 3. Separation of test mixture on a polymeric $C_{18}$ phase. Eluent is $100 \%$ methanol or $100 \%$ acetonitrile at a flow-rate of $1.5 \mathrm{ml} / \mathrm{min}$. For legend, see Fig. 2.

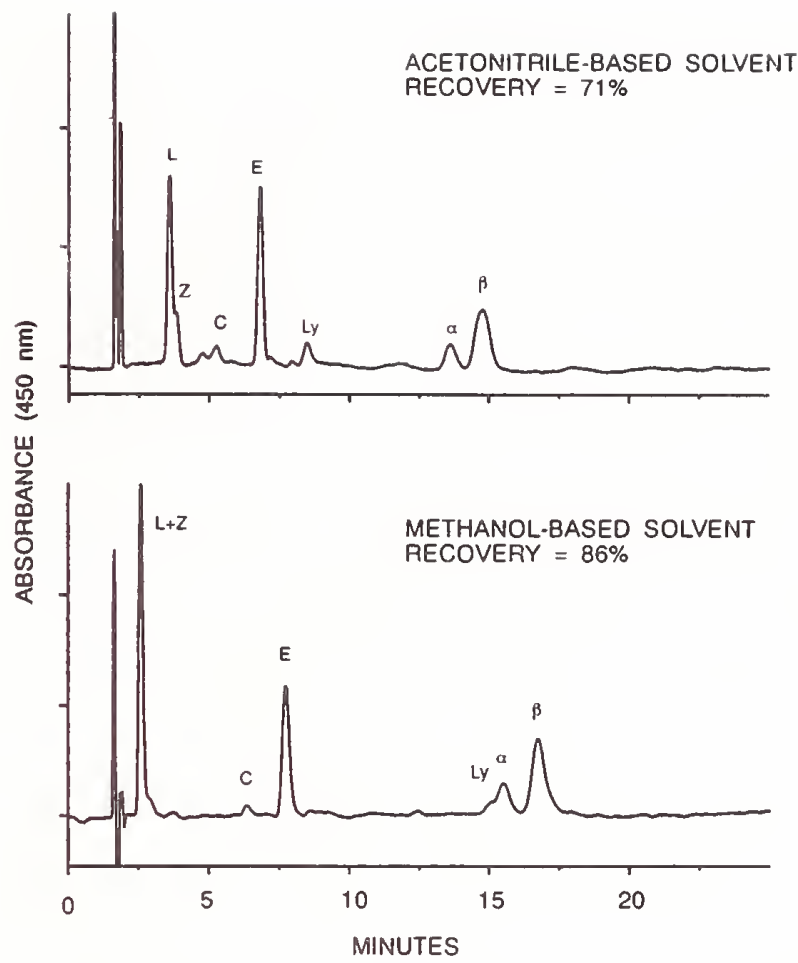

Fig. 4. Separation of test mixture on an intermediate $C_{18}$ phase. Eluent is THF-methanol (7.5:92.5) or THF-acetonitrile $(12.5: 87.5)$ at a flow-rate of $1.5 \mathrm{ml} / \mathrm{min}$. For legend, see Fig. 2.

\section{TABLE $1 \mathrm{I}$}

RESOLUTION $(R)$ AND SELECTIVITY ( $\alpha$ ) FOR ZEAXANTHIN/LUTEIN (Z/L) AND $\beta$-CAROTENE/LYCOPENE (B/Ly) ON SELECTED $C_{18}$ COLUMNS USING METHANOL AND THF-METHANOL COMPARED TO THE TBN/BaP $\propto$ VALUE ON THOSE COLUMNS

\begin{tabular}{|c|c|c|c|c|c|c|}
\hline Column ${ }^{a}$ & Character & $x_{Z / L}$ & $R_{\mathrm{Z} / \mathrm{L}}$ & $\alpha_{B / L y}$ & $R_{\mathrm{B} / \mathrm{L}, \mathrm{y}}$ & $\alpha_{\text {TBN/BaP }}$ \\
\hline 22 & Monomeric & 1 & 0 & 1.28 & 13 & 2.07 \\
\hline 14 & Monomeric & 1 & 0 & 1.20 & 6.2 & 2.05 \\
\hline 18 & Monomeric & 1 & 0 & 1.28 & 14 & 2.01 \\
\hline 25 & Monomeric & 1 & 0 & 1.18 & 7.8 & 2.00 \\
\hline 41 & Monomeric & 1 & 0 & 1.24 & 7.8 & 1.99 \\
\hline 44 & Monomeric & 1 & 0 & 1.29 & 13 & 1.97 \\
\hline 26 & Monomeric & 1 & 0 & 1.27 & 12 & 1.97 \\
\hline 40 & Monomeric & 1 & 0 & 1.29 & 12 & 1.95 \\
\hline 1 & Monomeric & 1 & 0 & 1.28 & 13 & 1.92 \\
\hline 20 & Monomeric & 1 & 0 & 1.19 & 7.4 & 1.84 \\
\hline 11 & Monomeric & 1 & 0 & 1.36 & 7.9 & 1.73 \\
\hline 53 & Intermediate & 1 & 0 & 1.15 & 10 & 1.68 \\
\hline 59 & Intermediate & 1 & 0 & 1.24 & 13 & 1.50 \\
\hline 19 & Intermediate & 1 & 0 & 2.16 & 19 & 1.45 \\
\hline 35 & Intermediate & 1.20 & 2.9 & 0.91 & 6.0 & 1.04 \\
\hline 30 & Polymeric & 1.18 & 2.9 & 0.78 & 8.0 & 0.93 \\
\hline 5 & Polymeric & 1.21 & 2.9 & 0.83 & 5.8 & 0.83 \\
\hline 6 & Polymeric & 1.23 & 4.0 & 0.74 & 12 & 0.78 \\
\hline 16 & Polymeric & 1.22 & 4.4 & 0.74 & 12 & 0.70 \\
\hline 49 & Polymeric & 1.26 & 3.9 & 0.70 & 16 & 0.59 \\
\hline 37 & Polymeric & 1.32 & 4.6 & 0.56 & 35 & 0.51 \\
\hline
\end{tabular}

\footnotetext{
a See Table 1 for column descriptions.
} 
tene. Using polymeric columns, the elution order often changed to $\alpha$-carotene, $\beta$-carotene, lycopene, thus the $\alpha$-values for the polymeric columns (and one intermediate column) in Table II are less than one. In general, if the column could resolve lutein and zeaxanthin, then lycopene eluted after $\alpha$ - and $\beta$-carotene. Quackenbush and Smallidge [54] and Bushway [35] have also observed that Vydac 201TP and $218 \mathrm{TP}$ columns, which are polymeric, reverse the order of elution of lycopene and $\beta$-carotene compared to other (monomeric) columns that they tested, although they did not recognize the monomeric or polymeric phase synthesis of the columns as the cause. Polymeric phases have also been reported to provide better separation of certain carotenoids and their cis isomers [35,54,55].

To investigate whether the selectivity for selected carotenoids correlates with the selectivities for the PAHs, $\alpha_{\text {TBN/BaP }}$ values for the columns are also shown in Table II. On the polymeric columns, $\alpha_{\mathrm{TBN} / \mathrm{BaP}}$ and $\alpha_{\text {zeaxanthin/lutein }}$ are correlated (correlation coefficient, $r=-0.94$ ), as are $\alpha_{\mathrm{TBN} / \mathrm{BaP}}$ and $\alpha_{\beta-}$ carotene/lycopene $(r=0.86)$. Despite the small sample size ( $n=6$ polymeric columns), there is more than $95 \%$ confidence that a correlation does exist, according to Pearson Product Moment correlation coefficient tables [56]. There is no correlation between the $\alpha_{\text {TBN/BaP }}$ and the $\alpha_{\text {zeaxanthin/lutein }}$ on the monomeric columns because all the $\alpha_{\text {zeaxanthin/lutein }}$ values are 1 , as is the case for most of the intermediate columns' $\alpha_{\text {zeaxanthin/lutein }}$ values. There is also no correlation between the $\alpha_{\mathrm{TBN} / \mathrm{BaP}}$ and the $\alpha_{\beta \text {-carotene/ly- }}$ copene on both the monomeric and intermediate columns. Thus, the $\alpha_{\mathrm{TBN} / \mathrm{BaP}}$ values are useful for predicting whether a column will be able to separate lutein and zeaxanthin inasmuch as it indicates whether the column is monomeric, polymeric, or intermediate, since mainly polymeric columns are capable of resolving this pair. However, it is not useful for predicting whether an intermediate column will be able to resolve lutein and zeaxanthin, nor can it predict how much separation of lycopene and $\beta$-carotene a monomeric or intermediate column is likely to provide.

For most columns it was necessary to add a mobile phase modifier to elute compounds with a $k^{\prime} \leqslant$ 11. The choice of modifier (THF or ethyl acetate) did not significantly affect selectivity for either lutein/zeaxanthin or lycopene/ $\beta$-carotene, the two pairs that were examined. Columns that failed to resolve lutein and zeaxanthin using methanol-based solvents failed using each modifier. On a few columns, the use of one modifier in acetonitrile did provide higher $\alpha$ values than the other, but the difference was not great enough to significantly improve resolution. THF did not consistently provide higher $\alpha$ values than ethyl acetate or vice versa. For the separation of lycopene and $\beta$-carotene, selectivity differed slightly using acetonitrile-based mobile phases on some columns. On one or two columns using methanol-based solvents, the $\alpha$ values for lycopene and $\beta$-carotene may have been more significantly affected by the choice of modifier. Again. THF was not consistently better or worse than ethyl acetate at providing greater resolution.

Pore size of the column can affect the selectivity for carotenoids of sufficiently different size (e.g., lycopene and $\beta$-carotene, zeaxanthin and $\beta$-carotene) but does not affect selectivities for carotenoids of very similar sizes (e.g., $\alpha$-carotene and $\beta$-carotene). In a comparison of four monomeric columns from the same manufacturer (column 45, 26, 27 and 28), the only difference being pore size of the silica substrate (i.e., 60, 120, 200 and $\lesssim 00 \AA$, respectively), both $\alpha_{\beta \text {-carotene/lycopene }}$ and $\alpha_{\beta \text {-carotene/zeaxanthin }}$ decrease with increasing pore size. This also holds true for a comparison of two other sets of wide-pore columns and their narrow-pore counterparts (columns 12 and $13,100 \AA$ vs. columns 14 and 15, $300 \AA$; column $36,120 \AA$ vs. column 37, $300 \AA$ ). In addition, as pore size decreases, more modifier must be added in all three cases to elute all seven compounds with a $k^{\prime} \leqslant 11$. This is as expected since the greater surface area of the base silica of the narrow-pore columns results in a higer carbon load. Although absolute retention of carotenoids increases with decreasing pore size (and increasing carbon loading), $\alpha$ values for similarly sized carotenoids do not change with column pore size. An example of this trend is the $\alpha$ values for $\beta$-carotene $/ \alpha$-carotene, which are the same for the sets of columns described above.

\section{Recovery}

Sample losses on the column are critical to the sensitivity and precision of an $\mathrm{LC}$ method and to quantitative analysis. Carotenoid recoveries ranging from 0 to almost $100 \%$ were observed. Recovery was dependent on the mobile phase, stationary phase type classification, and possibly the column bed support frit material. 
Mobile phase. On almost all the columns tested. the use of methanol or methanol-based solvents provided a higher recovery of the carotenoids than did the use of acetonitrile of acetonitrile-based solvents, as shown by the mean recoveries given in Table III. When using acetonitrile-based mobile phases, the THF modifier resulted in a higher percent recovery on most columns than did the ethyl acetate. This difference in recovery was less noticeable when the methanol-based eluents were used. Addition of a modifier was not necessary for about half the columns when methanol was used and about one-third of the columns when acetonitrile was used. Typically, it took less THF than ethyl acetate to elute the compounds with $k^{\prime} \leqslant 11$, and methanol required less modifier than acetonitrile, which is contrary to expectations based on solvent strength parameters, where acetonitrile is classified as a stronger solvent than methanol [57]. However, this observation is consistent with the findings of Nelis and De Leenheer, who reported that methanol acts as a stronger solvent than acetonitrile for the separation of carotenoids on $C_{18}$ columns [19]. Since recoveries were lower and no improvements in selectivity were observed, ethyl acetate-modified solvents are not discussed further to simplify data analysis. Henceforth each column has just two sets of results: $100 \%$ methanol and $100 \%$ acetonitrile, or THF-methanol and THF-acetonitrile if it was necessary to use a modifier.

\section{TABLE III}

AVERAGE RECOVERY OF CAROTENOIDS ON ALL COLUMNS TESTED USING DIFFERENT MOBILE PHASES

THF or ethyl acetate are added to methanol and acetonitrile as modifiers such that $7 \leqslant k_{\max }^{\prime} \leqslant 11$.

\begin{tabular}{ll}
\hline Mobile phase & Recovery \pm S.D. $(\%)$ \\
\hline $100 \%$ methanol & $84 \pm 8(n=29)^{a}$ \\
Methanol-THF & $86 \pm 11(n=35)$ \\
Methanol-ethyl & $82 \pm 12(n=35)$ \\
acetate & \\
$100 \%$ Acetonitrile & $56 \pm 19(n=21)$ \\
Acetonitrile-THF & $68 \pm 17(n=43)$ \\
Acetonitrile-ethyl & $47 \pm 17(n=43)$ \\
acetate & \\
\hline
\end{tabular}

${ }^{a} n=$ Number of columns.
Acetonitrile-based solvents are typically used for the carotenoid separations reported in the literature [3-12,14,17-20,22,25,26,29-38,40-48]. However, our studies show that recoveries using acetonitrile and acetonitrile-based eluents are generally lower than those obtained using methanol and methanolbased eluents. Nelis and De Leenheer [19] reported that the incorporation of methanol in acetonitrilebased solvents dramatically enhanced selectivity, which they speculated was due to hydrogen bonding. Most of the acetonitrile-based methods reported in the literature do involve the use of some methanol $[3,6,7,9-11,14,17,19,20,22,26,29-35,37,38,40-$ $44.46-48]$. To determine whether the addition of methanol improved recovery as well as selectivity, a column that had provided $0 \%$ recovery when run with $100 \%$ acetonitrile and $77 \%$ recovery with $100 \%$ methanol was tested with a methanol-acetonitrile (10:90) mixture. Recovery with respect to the $100 \%$ acetonitrile run improved only slightly (up from 0 to $2 \%$ ). A run using methanol-acetonitrile $(20: 80)$ as the eluent resulted in $4 \%$ recovery. Thus the addition of methanol did improve recovery marginally. The column tested performed poorly using $100 \%$ acetonitrile; it would be unfair to say that recovery on all columns using acetonitrilebased solvents would also show only a $2 \%$ increase in recovery with the addition of methanol.

The poor performance of certain columns appears to be real and not an artifact. When poorly performing columns were rechecked at a later date, performance had not changed. In addition, recovery was not affected when various sample sizes were injected (from $5 \mu \mathrm{l}$ to $100 \mu \mathrm{l}$ ). Lauren and $\mathrm{McNaughton}[50]$ recommend the addition of $0.1 \%$ $n$-decanol to the mobile phase to improve performance by minimizing adsorption. We did not find $n$ decanol to be effective in improving the performance of columns with poor recoveries using acetonitrile-based solvents. However, we have observed that flushing the column with an ammonium acetate buffer improves recovery on these columns when an acetonitrile-based mobile phase is used. We have also found that the addition of ammonium acetate to the mobile phase improves recoveries.

To determine the reproducibility of recovery results, one column from each of the three classification groups was retested about four months after the initial test. Columns that had required 100\% 
methanol were selected to eliminate any effect caused by slight variations in the mobile phase composition. Columns were also selected that had provided recoveries greater than $80 \%$ in the first test. Relative standard deviations ranged from 2.5 to $5.6 \%$. An insufficient number of runs were made on individual columns to allow statistical comparisons of methanol or methanol-based and acetonitrile or acetonitrile-based recoveries for each column; however, based on the reproducibility studies, $6 \%$ represents a liberal estimate of the relative standard deviation. Using this estimate of variability, columns with a $12 \%$ difference in recovery between methanol- and acetonitrile-based eluents would be significantly different. Columns are grouped according to their recoveries using methanol or THFmethanol and acetonitrile or THF-acetonitrile in Table IV.

Selective recovery. Experiments were performed to determine whether certain carotenoids contributed a greater share to recovery losses than did the other carotenoids. Individual carotenoids were injected into the system, both with and without a col-

\section{TABLE IV}

COLUMNS USED IN THIS STUDY GROUPED ACCORDING TO PERCENT RECOVERIES

\begin{tabular}{|c|c|}
\hline $\begin{array}{l}\text { Recovery } \\
(\%)\end{array}$ & Columns \\
\hline \multicolumn{2}{|c|}{ Using methanol or THF-methanol: } \\
\hline $90-100$ & $\begin{array}{l}35711152123242627282930324142434445 \\
5265\end{array}$ \\
\hline $80-89$ & $\begin{array}{l}12468101213141617181922253135373839 \\
40464748495051535458596061\end{array}$ \\
\hline $70-79$ & 203336556364 \\
\hline $60-69$ & 4262 \\
\hline $50-59$ & 9 \\
\hline $40-49$ & - \\
\hline $30-39$ & 56 \\
\hline \multicolumn{2}{|c|}{ Using acetonitrile or $T H F$-acetonitrile: } \\
\hline $90-100$ & 435051 \\
\hline $80-89$ & 23232737496061 \\
\hline $70-79$ & 148121318212226303241424547525859 \\
\hline $60-69$ & 5102528293336444865 \\
\hline $50-59$ & 911141517202431384046 \\
\hline $40-49$ & 67161935396264 \\
\hline $30-39$ & 63 \\
\hline $20-29$ & - \\
\hline $10-19$ & - \\
\hline $0-9$ & 53545556 \\
\hline
\end{tabular}

umn, as described. Two monomeric-phase and three polymeric-phase columns were tested. All columns had previously required $100 \%$ methanol for elution of the carotenoids in less than 25 min. Four of the columns had required $100 \%$ acetonitrile; one required $\mathrm{THF}$-acetonitrile (5:95). Two trends in recovery were observed. Losses of zeaxanthin and $\beta$-carotene, which each contain two $\beta$-rings (Fig. 1), were greater than losses of lutein and $\alpha$-carotene, which each contain one $\beta$ - and one $\varepsilon$-ring. Within the group of $\beta, \beta$-carotenoids used in the mixture (zeaxanthin, $\beta$-cryptoxanthin, echinenone, and $\beta$-carotene), recovery increased as polarity decreased: less zeaxanthin, a dihydroxy carotenoid, was recovered than $\beta$-cryptoxanthin, a monohydroxy carotenoid. Less $\beta$-cryptoxanthin was recovered than echinenone, a mono-keto carotenoid. And less echinenone was recovered than $\beta$-carotene, a hydrocarbon carotenoid. This trend also holds true for the two $\beta, \varepsilon$-carotenoids used in the mixture. The recovery of the dihydroxy carotenoid, lutein, was less than that of the hydrocarbon carotenoid, $\alpha$-carotene.

Stationary phase. In these carotenoid studies, as a rule, polymeric phases required the least modifier (a mean value of $2 \%$ THF in methanol, $3 \%$ THF in acetonitrile) and monomeric phases required the most (a mean value of $6 \% \mathrm{THF}$ in methanol, $9 \%$ $\mathrm{THF}$ in acetonitrile) to elute all seven compounds from the column within the $k^{\prime}$ range allowed $\left(7 \leqslant k^{\prime} \leqslant 11\right)$. Intermediate phases required an intermediate amount of modifier ( $4 \%$ THF in methanol, $6 \% \mathrm{THF}$ in acetonitrile). Percent recovery across the three groups did not vary consistently. Recovery results for columns divided into the three classification groups are shown in Table V. Thirty percent of the columns gave $>90 \%$ recovery using

\section{TABLE V}

AVERAGE PERCENT RECOVERY FOR POLYMERIC, INTERMEDIATE, AND MONOMERIC COLUMNS TESTED

\begin{tabular}{llll}
\hline Eluent & Polymeric & Intermediate & Monomeric \\
\hline $\mathrm{MeOH}^{a}$ & $83 \%$ & $79 \%$ & $88 \%$ \\
$\mathrm{ACN}^{b}$ & $62 \%$ & $46 \%$ & $67 \%$ \\
\hline
\end{tabular}

${ }^{a} \mathrm{MeOH}=$ methanol and methanol-based eluents.

${ }^{b} \mathrm{ACN}=$ acetonitrile and acetonitrile-based eluents. 
methanol or THF-methanol as the solvent; of these, $70 \%$ were classified as monomeric and the remaining $30 \%$ were classified as polymeric.

In several cases, columns were available that contained the "same" packing material from different lots. Recovery results and $k^{\prime}$ values for selected compounds using THF-methanol for columns from different production lots are presented in Table VI. Mean recoveries for many of the columns appear to be different, but performance of Tukey's Multiple Comparison test [58] on these data shows that there is no difference between the mean recoveries at a $95 \%$ confidence level for almost all the column pairs.

Retention times of carotenoids using columns from different stationary phase lots varied substantially. (On one pair of columns, there was a $15 \%$ difference in $\beta$-carotene's retention time.) This variability can arise from two sources: the base silica from which the stationary phase is prepared and reproducibility of the bonding procedure. Most LC column manufacturers obtain silica from outside suppliers, and differences between column lots is strongly related to differences in the silica-particle size, pore size, surface area. silanol activity. and trace metal contamination. Thus, column lot differences can exist for both monomeric and polymeric phases. The only way to ensure reproducible columns is to purchase columns packed with the same production lot of stationary phase.

Column bed support frits. It has been suggested that losses occurring on column frit materials may be partially responsible for low recoveries of carotenoids $[3,23]$. Data from two sets of columns that were identical except for the presence of different frits are presented in Table VII. Although recoveries were slightly lower for stainless steel frits, no significant differences in recoveries between these sets of columns were detected. Later studies in which frits were placed in series (without a column in the system) showed no significant difference in recovery using stainless steel, titanium, or "biocompatible" frits.

In 1986, Nierenberg and Lester [3] observed dif-

TABLE VI

COMPARISON OF $k^{\prime}$ AND RECOVERY FOR COLUMNS FROM DIFFERENT LOTS

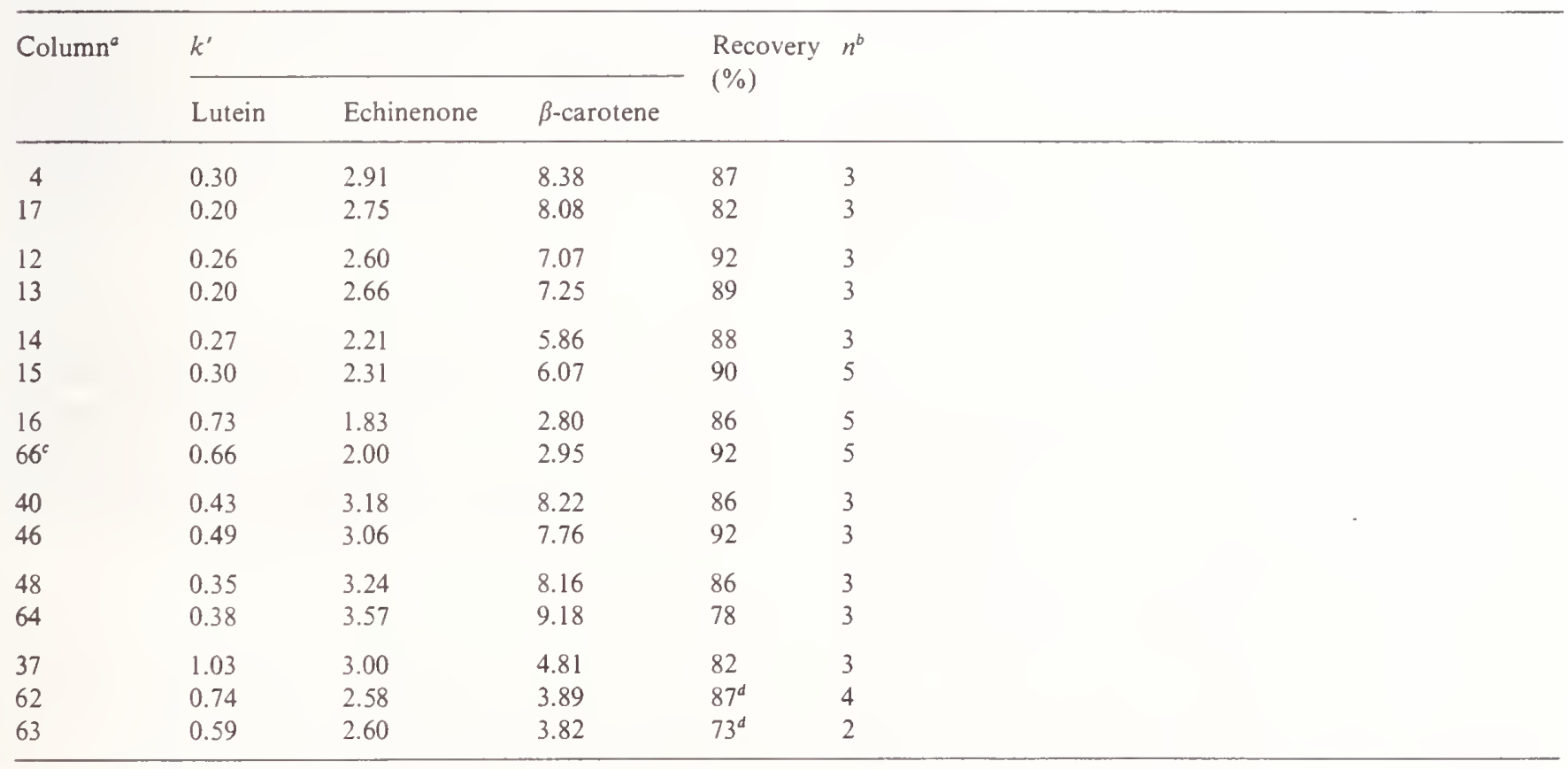

- See Table I for column descriptions.

b $n=$ Number of runs.

c Supelco LC-PAH column tested after the completion of the other analyses.

"Statistical difference in recovery as determined by Tukey's Multiple Comparisons. 
TABLE VII

COMPARISON OF $k^{\prime}$ AND RECOVERIES ON COLUMNS WITH DIFFERENT FRIT MATERIALS

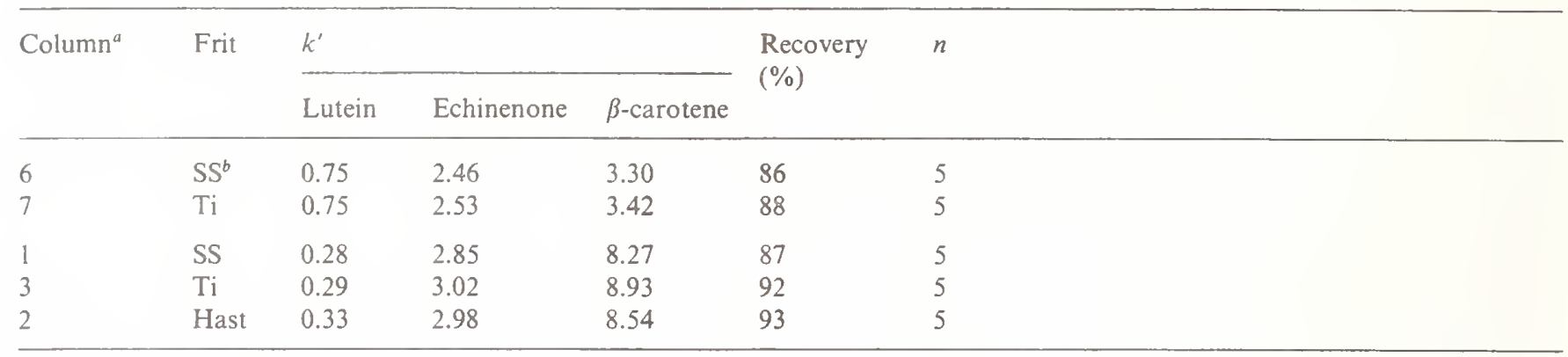

a See Table I for column description.

${ }^{b} \mathrm{SS}=$ Stainless steel: $\mathrm{Ti}=$ titanium; Hast $=$ Hastelloy .

ferences in recovery when they switched to a new column of the same brand from the same manufacturer, but with a different kind of frit, and they attributed these differences to the use of different frits (stainless steel $v s$. Hastelloy). They also observed this phenomenon when two columns were packed with the same lot of packing material, topped with either stainless steel or Hastelloy frits. During our studies, it was observed that a certain previously unused column did not provide the same recovery or retention times as its older, used, counterpart, but that the "new" column gradually attained the retention and recovery characteristics of the "old" column with repeated use. Columns that had been used in our laboratory prior to this study have been marked in Table I, in the event that results for these columns have been affected by previous use in the laboratory.

\section{CONCLUSIONS}

Several conclusions concerning the selectivity and recovery of carotenoids in reversed-phase LC can be drawn from this study: (1) In general, separations using acetonitrile or the frequently employed acetonitrile-based solvents resulted in lower recoveries than separations using methanol or methanol-based solvents. (2) Monomeric $\mathrm{C}_{18}$ columns generally provided high recoveries, but they were unable to resolve lutein and zeaxanthin using methanol or methanol-based eluents, which were the solvents yielding the highest recoveries. (3) Polymeric $\mathrm{C}_{18}$ columns were usually able to resolve lutein and zeaxanthin in methanol-based solvents, but on average had lower recoveries than monomeric $\mathrm{C}_{18}$ columns. (4) Using methanol-based solvents, the retention time of lycopene is greater than that of $\beta$-carotene on most polymeric columns. (5) Columns with the "same" stationary phases from different lots do not necessarily elute compounds with the same retention times. (6) When using a new column, it may be necessary to make several preliminary runs before the separation (i.e., retention times, recovery, selectivity) becomes reproducible and comparable to that expected based on the performance of the corresponding "old" column. (7) The most appropriate system for the separation of carotenoids would probably include the use of a polymeric $\mathrm{C}_{18}$ column (to allow separation of lutein and zeaxanthin) and a methanol-based mobile phase (to obtain a high percent recovery).

\section{ACKNOWLEDGEMENTS}

This work was supported in part by the National Cancer Institute, Division of Cancer Etiology, Agreement No. Y01-CP9-0513. We extend our thanks to the many companies who supplied us with their columns for this study.

\section{REFERENCES}

1 R. Peto, R. Doll, J. D. Buckley, and M. B. Sporn, Nature, (London), 290 (1981) 201.

2 R. G. Ziegler, Am. J. Clin. Nutr., 53 (1991) $251 \mathrm{~S}$.

3 D. W. Nierenberg and D. C. Lester, J. Nutr. Growth Cancer, 3 (1986) 215.

4 W. J. Driskell, J. W. Neese, C. C. Bryant and M. M. Bashor, J. Chromatogr., 231 (1982) 439. 
5 W. J. Driskell, M. M. Bashor and J. W. Neese, Clin. Chem. 29 (1983) 1042.

6 Y. M. Peng, J. Beaudry, D. S. Alberts and T. P. Davis, J. Chromatogr., 273 (1983) 410.

7 C. C. Tangney, J. Liq. Chromatogr., 7 (1984) 2611.

8 W. J. Driskell, A. D. Lackey, J. S. Hewett and M. M. Bashor, Clin. Chem., 31 (1985) 871.

9 K. W. Miller and C. S. Yang, Anal. Biochem., 145 (1985) 21.

10 D. A. Kalman, G. E. Goodman, G. S. Omenn, G. Bellamy and B. Rollins, J. Natl. Cancer Inst., 79 (1987) 975.

11 C. R. Broich, L. E. Gerber and J. W. Erdman, Jr., Lipids, 18 (1983) 253.

12 N. Katrangi, L. A. Kaplan and E. A. Stein, J. Lipid Res., 25 (1984) 400

13 K. W. Miller, N. A. Lorr and C. S. Yang, Anal. Biochem., 138 (1984) 340 .

14 D. W. Nierenberg, J. Chromatogr., 339 (1985) 273.

15 D. B. Milne and J. Botnen, Clin. Chem., 32 (1986) 874.

16 V. J. Gatautis and K. H. Pearson, Clin. Chim. Acta, 166 (1987) 195.

17 Y. Ito, R. Sasaki, M. Minohara, M. Otani and K. Aoki, Clin. Chim. Acta, 169 (1987) 197.

18 G. Wang, M. M. Root, X. Ye, J. Chen and T. C. Campbell, J. Micronutr. Anal., 5 (1989) 3.

19 H. J. C. F. Nelis and A. P. De Leenheer, Anal. Chem., 55 (1983) 270.

20 J. G. Bieri, E. D. Brown and J. C. Smith, Jr., J. Liq Chromatogr., 8 (1985) 473.

21 R. Ohmacht, Gy. Tóth and G. Voigt, Chromatographia, 22 (1986) 189.

22 M. Stacewicz-Sapuntzakis, P. E. Bowen. J. W. Kikendall and M. Burgess, J. Micronutr. Anal., 3 (1987) 27.

23 W. A. MacCrehan and E. Schönberger, Clin. Chem., 33 (1987) 1585.

24 R. Ohmacht, Gy. Tóth and G. Voigt, J. Chromatogr., 395 (1987) 609.

25 L. A. Kaplan, J. A. Miller and E. A. Stein, J. Clin. Lab. Anal., 1 (1987) 147.

26 C. S. Yang and M. J. Lee, J. Nutr. Growth Cancer, 4 (1987) 19.

27 A. L. Sowell, D. L. Huff, E. W. Gunter and W. J. Driskell, J. Chromatngr.. 431 (1988) 424.

28 G. Cavina, B. Gallinella, R. Porra, P. Pecora and C. Suraci, J. Pharm. Biomed. Anal., 6 (1988) 259.

29 L. R. Cantilena and D. W. Nierenberg, J. Micronutr. Anal., 6 (1989) 127

30 A. B. Burua, R. O. Batres, H. C. Furr and J. A. Olson, J. Micronutr. Anal., 5 (1989) 291.
31 B. Olmedilla, F. Granado, E. Rojas-Hidalgo and 1. Blanco, J. Liq. Chromatogr., 13 (1990) 1455.

32 A. J. Culling-Berglund, S. A. Newcomb, M. Gagne, W. S. Morfitt and T. P. Davis, J. Micronutr. Anal., 5 (1989) 139.

33 Y. Kim, C. English, P. Reich, L. E. Gerber and K. L. Simpson, J. Agric. Food Chem., 30 (1990) 1930.

34 J. C. Bauernfeind, J. Agric. Food Chem., 20 (1972) 456.

35 R. J. Bushway, J. Liq. Chromatogr., 8 (1985) 1527.

36 J. L. Bureau and R. J. Bushway, J. Food Sci., 51 (1986) 128.

37 F. Khachik, G. R. Beecher, and N. F. Whittaker, J. Agric. Food Chem., 34 (1986) 603.

38 J. F. Fisher and R. L. Rouseff, J. Agric. Food Chem., 34 (1986) 985

39 C. Fisher and J. A. Kocis, J. Agric. Food Chem., 35 (1987) 55.

40 P. W. Simon and Z. Y. Wolff, J. Agric. Food Chem., 35 (1987) 1017.

41 F. Khachik and G. R. Beecher, J. Chromatogr., 449 (1988) 119

42 F. Khachik and G. R. Beecher, J. Agric. Food Chem., 36 (1988) 929.

43 S. Scalia and G. W. Francis, Chromatographia, 28 (1989) 129.

44 B. Czinkotai, H. G. Daood, P. A. Biacs and F. Hajdu, J. Liq. Chromatogr., 12 (1989) 2707.

45 P. A. Biacs, H. G. Daood, A. Pavisa and F. Hajdu, J. Agric. Food Chem., 37 (1989) 350.

46 M. I. Heinonen, V. Ollilainen, E. K. Linkola, P. T. Varo and P. E. Koivistoinen, J. Agric. Food Chem., 37 (1989) 655.

47 F. Khachik, G. R. Beecher and W. R. Lusby, J. Agric. Food Chem., 37 (1989) 1465.

48 M. I. Heinonen, J. Agric. Food Chem., 38 (1990) 609.

49 M. Zakaria, K. Simpson, P. R. Brown and A. Krstulovic, J. Chromatogr., 176 (1979) 109.

50 D. R. Lauren and D. E. McNaughton, J. Liq. Chromatogr., 9 (1986) 2013.

51 L. C. Sander and S. A. Wise, LC. GC, 5 (1990) 378.

52 G. Britton, Methods Enzymol., 111 (1985) 113.

53 Certificate of Analysis, SRM 869. Column Selectivity Test Mixture for Liquid Chromatography, National Institute of Standards and Technology, Gaithersburg, MD, 1990.

54 F. W. Quackenbush and R. L. Smallidge, J. Assoc. Off. Anal. Chem., 69 (1986) 767.

55 N. E. Craft, L. C. Sander and H. F. Pierson, J. Micronutr. Anal., 8 (1991) 209.

56 N. R. Ullman, Elementary Statistics, Wiley, New York, 1978.

57 L. R. Snyder, Introduction to Modern Liquid Chromatography, Wiley, New York 1979.

$58 \mathrm{H}$. Schessé, The Analysis of Variance, Wiley, Chichester, 1959. 


\title{
Liquid chromatographic method for the determination of carotenoids, retinoids and tocopherols in human serum and in food
}

\author{
Katherine S. Epler \\ Organic Analytucal Research Division. Chemical Science and Technology Lahoratort. Vational Instutute of Sundards and Technolesgl: \\ (SS Department of Commerce. Technology Administration. Gathershurg. WD 20899 ( (SA)
}

\section{Regina G. Ziegler}

Environmental Epidemiolog. Branch. Epidemiolog.' and Biostatistics Program. Division of Cancer Elinlog!. Yational Cancer Institutt' Bethesda. HD 2089? ( (LS.4)

Neal E. Craft

Organic Analytical Research Division. Chemical Science and Technology Laboratory. National Institute of Standards and Technology. L'.S. Department of Commerce. Technolog. Administration. Gaithersburg. MD 20899 (LSA)

(First received April 2nd. 1993: revised manuscript received May 31st. 1993)

\begin{abstract}
A liquid chromatographic (LC) method has been developed for the quantitative measurement of the six major carotenords in human serum (lutein, zeaxanthin. $\beta$-cryptoxanthin. lycopene, $x$-carotene, and $\beta$-carotene) as well as retinol, retinyl palmitate. $x$-tocopherol. $\gamma$-tocopherol, and $\delta$-tocopherol. Several polar carotenoids, $2^{\prime} \cdot 3^{\prime}$-anhydrolutein. $x$-cryptoxanthin. and geometric isomers of licopene and $\beta$-carotene are also separated. Retinoids and carotenoids are monitored using a programmable ultraviolet-visıble detector. uhile tocopherols are monitored usıng a fluorescence detector. The method uses a gradient containıng acetonitrile, methanol. and ethyl acetate. Ammonium acetate is introduced with the methanol to minimize carotenoid losses on the LC column aggravated by the use of acetonitrile and ethyl acetate. The method is also applicable to the analysis of foods.
\end{abstract}

\section{INTRODUCTION}

Epidemiologists have observed a reduced risk of lung cancer in people who have an elevated intake of fruits and vegetables [1]. Epidemiologic studies also suggest that eating fruits and vegetables may reduce the risk of other cancers as well [1]. Because low serum levels of $\beta$-carotene are associated with the subsequent development of

\footnotetext{
* Corresponding author.

* Present address: Southern Testing and Research Laboratories. Wilson, NC 27893, USA.
}

lung cancer, $\beta$-carotene may be the protective factor present in the fruits and vegetables [1]. However, the lowered risk of cancer may be due to other carotenoids that are ingested along with the $\beta$-carotene, and the importance of these other carotenoids has not been studied adequately.

Six carotenoids (lutein, zeaxanthin. $\beta$-cryptoxanthin, lycopene, $x$-carotene, and $\beta$-carotene) reportedly account for more than $90 \%$ of the carotenoids present in the serum of American populations [2]. The goal of this study was to separate and measure the six major carotenoids in serum (as well as some of the geometric isomers

0378-4347;93/\$06.00 C 1993 Elsevier Science Publishers B.V. All rights reserved 
of these carotenolds), retinol. tocopherols. and retinyl palmitate. The reversed-phase liquid chromatographic (LC) method that has been developed allows quantitation of retinol. retinyl palmitate. lutein. zeaxanthin. $\beta$-cryptoxanthin. truns-lycopene. total lycopene. trans-x-carotene. total $\alpha$-carotene. truns- $\beta$-carotene. total $\beta$-carotene. $\because-$ tocopherol. $j$-tocopherol. and $z$-tocopherol. The method also resolves $x$-cryptoxanthin. 2.3 anhydrolutein. and the cis isomers of lycopene. $x$-carotene. and $\beta$-carotene. as well as some unidentified polar carotenoids. Most published methods use reversed-phase LC to separate major carotenoids but do not resolve geometric isomers [2-9]. Some workers do report the separation of geometric isomers. usually those of $\beta$-carotene. when measuring the major carotenoids $[10-12]$. Other workers have concentrated on the separation of just $\beta$-carotene isomers [13.14] or isomers of both $x$ - and $\beta$-carotene $[15.16]$. To measure serum levels of each major carotenoid accurately, it is important that isomers of that carotenoid not interfere with the measurement of the isomer of interest. otherwise that measurement should be identified as a "total" measurement. i.e.. "total $\beta$-carotene" as opposed to "trans- $\beta$-carotene" or the somewhat indefinite " $\beta$-carotene." The ideal method would separate all the carotenoids and geometric isomers of interest in the serum as well as the retinoids and tocopherols. Of course. it is difficult to know whether all the isomers are separated; if a method cannot separate two isomers. it is not possible to tell that there are two isomers present merely by looking at a chromatogram that has resulted from the measurement of UV absorbance at a single wavelength.

In work similar to this study. Hess et al. [17] separated retinol. the tocopherols. $\beta$-cryptoxanthin. lycopene. $x$-carotene. $\beta$-carotene. and some isomers in human serum. and measured them with both UV absorbance and fluorescence: however. they were unable to resolve lutein and zeaxanthin. Using the method described here. we have analyzed standard reference material (SR.M) 968a. fat-soluble vitamins in human serum. for the compounds listed above. Although $x$-cryptoxanthin. 2'. $3^{\prime}$-anhydrolutern. and cis isomers of lycopene. $x$-carotene. and $\beta$-carotene are resolved from the other components using this method. standards were not available to permit quantitation of these compounds. We have also demonstrated the method's applicability to food by analyzing a mixture of foods.

EXPERIME\TAL ${ }^{*}$

\section{Internal standards}

Tocol (Esai. Tokyo. Japan) was used as the internal standard for the tocopherols. $\operatorname{tr}$ an.s- $\beta$ Apo-10'-carotenal oxime. the internal standard for the retinoids and carotenoids. was prepared from trans- $\beta$-apo-10'-carotenal (Hoffman-LaRoche. Basel. Switzerland) [18]. The oxime was purified by passing a concentrated solution through a preparative $V y d a c 201 T P$ column using 50:50 methanol-acetonitrile and collecting the fraction that contained the trans- $\beta-$ apo- $10^{\prime}$. carotenal oxime. The resulting solution was $99.6 \%$ pure.

\section{Rerinol and carotenoids}

Three calibration standards containing known low. intermediate. and high levels ( relative to the physiological levels in serum) of lutein (Kemın Industries. Des Moines. (A. LiSA). zeaxanthin and $\beta$-cryptoxanthin (Atomergic Chemetals. Farmingdale. NY. USA), and retinol. $x-$ and $\beta$-carotene (Sigma. St. Louis. MO. LSA) were prepared in ethanol with $30 \mu \mathrm{g} \mathrm{ml}$ butylated hydroxytoluene (BHT. an antioxidant) added. Structures of retinol. these carotenoids. and trans- $\beta$-apo-10'-carotenal oxime are shown in Fig. 1. Stock solutions from which standards were prepared were filtered through $45-\mu \mathrm{m}$ PTFE syringe filters. Concentrations of these stock solutions were determined spectrophotometrically.

\footnotetext{
- Certain commercial equipment. instruments. or materiats dre identulied in this report to specify adequately the experimental procedure. Such identitication does not imply recommendittion or endorsement by the National Institute of Standard, and Technology. nor does it imply that the materials or eyuipment identified are necessarily the best avalable for the purpose
} 


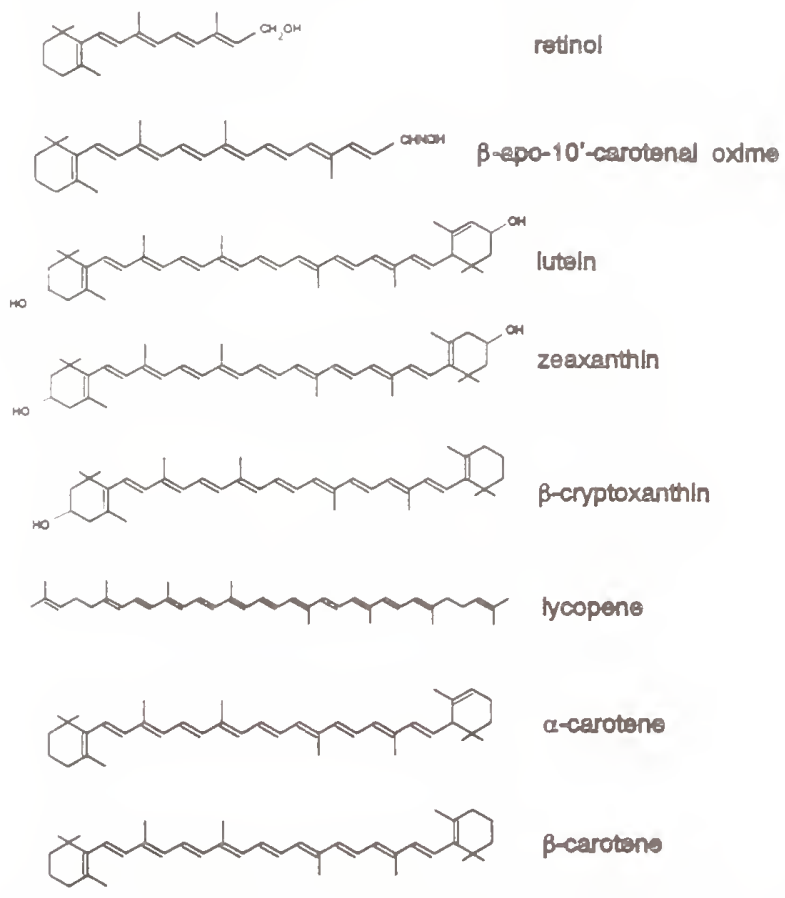

Fig. I. Structures of retinol, trans- $\beta$-apo-10'-carotenal oxime. and the carotenoids measured using this method.

and LC analysis of the stock solutions was performed to determine the purity of each solution. Absorptivities and maximum wavelengths used for calibration are shown in Table I. The calibration curve was prepared immediately following preparation of the standards. A l-ml volume of each calibration solution was combined with

TABLE I

ABSORPTIVITIES AND MAXIMUM WAVELENGTHS USED FOR CALIBRATION

\begin{tabular}{lll}
\hline Compound & Absorptivity $(\mathrm{d} / \mathrm{g} \cdot \mathrm{cm})$ & $i_{\max }(\mathrm{nm})$ \\
\hline Retınol & 1850 & 325 \\
Lutein & 2765 & 445 \\
Zeaxanthin & 2416 & 452 \\
$\beta$-Cryptoxanthin & 2486 & 452 \\
trans-Lycopene & 3450 & 472 \\
trans-x-carotene & 2800 & 444 \\
trans- $\beta$-Carotene & 2592 & 452 \\
Retunyl palmitate & 975 & 325 \\
$\delta$-Tocopherol & 91.2 & 297 \\
$\because$-Tocopherol & 91.4 & 298 \\
$x$-Tocopherol & 75.8 & 292 \\
\hline
\end{tabular}

$200 \mu \mathrm{l}$ of internal standard solution, and the resulting solution was placed in a glass insert in an autosampler vial. The autosampler. which held the samples at $15^{\circ} \mathrm{C}$, injected a $20-\mu$ l aliquot of the solution onto the column.

Due to rapid degradation, three lycopene (Sigma) calibration solutions were prepared separately for immediate use. These solutions were also prepared in ethanol with $30 \mu \mathrm{g}, \mathrm{ml} \mathrm{BHT}$ added. and $1 \mathrm{ml}$ of each solution was mixed with 200 $\mu \mathrm{l}$ of internal standard solution.

\section{Tocopherols and resinil palmitare}

Solutions containing low, intermediate. and high levels of $x-, \delta$-, and $;-$ tocopherol (Eastman Kodak, Rochester, NY, USA) and retinyl palmitate (Sigma) were prepared as described above. Structures of retinyl palmitate. tocol. $x$-tocopherol, $\dot{\delta}$-tocopherol, and $;$-tocopherol are show $n$ in Fig. 2.

\section{Serum extracts}

SRM 968a. fat-soluble vitamins in human serum (Standard Reference Materials Program. National Institute of Standards and Technology, Gaithersburg, MD. USA), was reconstituted with $1.0 \mathrm{ml}$ of HPLC-grade water. Duplicate

$$
\text { Retinyl palmitate }
$$

Fig. 2. Structures of retinyl palmitate, tocol. and $x-, ;-$. and $j$-tocopherol. 
200- $\mu$ l aliquots of the reconstituted serum were placed in glass test tubes. and serum proteins were denatured with an equal volume of ethanol containing the internal standards and BHT. The samples were mixed on a vortex mixer for $15 \mathrm{~s}$. Hexane $(1 \mathrm{ml})$ was added. and the mixtures were placed on a vortex mixer for $45 \mathrm{~s}$. Then the samples were centrifuged ( $30 \mathrm{~s}$. $2000 \mathrm{~g}$ ). and the supernatants were removed. The hexane extraction process was repeated. and the supernatant from this second extraction was combined with that of the first. Extracts were evaporated under a stream of nitrogen and reconstituted in $100 \mu \mathrm{l}$ of ethanol containing $30 \mu \mathrm{gl} \mathrm{BHT}$. The reconstituted extracts were placed in an ultrasonicating bath for $30-60$ s to facilitate dissolution. The extracts were then transterred to glass inserts that were placed in the autosampler vials.

\section{Quality conerol}

Two sets of serum samples containing low and normal levels of retinol. carotenoids. and tocopherols were run fifteen times over a five-week period to ascertain that the method was in control and to determine the reproducibility of the method. A low and a normal sample were run each day. Peak-area measurements were used for all nine analytes: retinyl palmitate was not measured in these samples.

\section{Food extracts}

An extract of a mixture of foods containing apricots. carrots. creamed corn. creamed peas. green beans. orange juice, peaches. spinach. sweet potatoes. tomato paste. and winter squash was analyzed using the method described below to demonstrate the method's applicability to food analysis.

\section{Chromatographic conditions}

The method involved two linear gradients and an isocratic step. Solvent. A was acetonitrile. solvent $\mathrm{B}$ was methanol containing $0.05 . \mathrm{M}$ ammonium acetate. and solvent $C$ was ethyl acetate. Each of the three solvents contained $0.05 \%$ triethylamine (TE.A) for reasons that are discussed below. The first linear gradient began with $98 \% \mathrm{~A}-2 \% \mathrm{~B}$ and went to $75 \% \mathrm{~A}-18 \% \mathrm{~B}-7 \% \mathrm{C}$ in $10 \mathrm{~min}$. The second linear gradient ran from this composition to $68 \% \mathrm{~A}-25{ }^{\circ}$ 。 $\mathrm{B}-7 \% \mathrm{C}$ in five $\mathrm{min}$. This composition was held for $10 \mathrm{~min}$ longer. then the system was returned to initial conditions $\left(98^{\circ} \circ \mathrm{A}-22^{\circ} \circ \mathrm{B}\right)$ over $5 \mathrm{~min}$ and re-equilibrated for $10 \mathrm{~min}$.

The precolumn $110 \mathrm{~mm} \times 4.6 \mathrm{~mm} \mathrm{I.D..} 10 \mu \mathrm{m}$ particle size) containing Vydac 201TP polymerıc wide-pore $C_{1 \text { s }}$ (Separations Group. Hesperia. CA. L'SA) stationary phase and the narrow-pore Bakerbond $C_{18}$ analytical column $1250 \mathrm{~mm} \times$ $4.6 \mathrm{~mm}$ I.D. $5 \mu \mathrm{m}$ particle size. J. T. Baker. Phillipsburg. NJ. (SA) were held at $29=0.5^{\circ} \mathrm{C}$ by a thermostatted water bath. In previous work. column temperature has been shown to influence selectivity for carotenoids and other compounds with molecule shape differences [19]. The stainless-steel frits throughout the chromatographic system were replaced with biocompatuble frits [20]. A programmable L'V-Vis detector (Spectroflow 783. ABI. Foster City. CA. (SA) with a tungsten lamp was used for measurement of the retinoids and the carotenoids. The wavelength was held at $325 \mathrm{~nm}$ to measure retinol and then was changed to $450 \mathrm{~nm}$ at 4.5 min to measure the internal standard and the carotenoids: the wavelength was changed back to $325 \mathrm{~nm}$ at $22 \mathrm{~min}$ to measure retinyl palmitate. A Huorescence spectrometer (LS-4. Perkin-Elmer. Norwalk. CT. USA) was used to measure the tocopherols and tocol using an excitation wavelength of $295 \mathrm{~nm}$ and an emission wavelength of $335 \mathrm{~nm}$. Signals from both detectors were recorded simultaneously by the data system (Maxima 820. Waters. Dirision of Millipore. Milford. MA. LSA).

\section{RESULTS AND DISCLSSION}

\section{Method development}

In a previous paper. we reported on a comparison of carotenoid separations on sixty-five reversed-phase LC columns [20]. Columns were classified as monomeric. polymeric. or intermediate based on their selectivity for a test mixture of polycyclic aromatic hydrocarbons (P.AHs). standard reference material 869 [21]. The selectivity test of Sander and Wise classifies phases as 
monomeric. intermediate, or polymeric depending on the elution order of benzo[a]pyrene ( $\mathrm{BaP})$ and tetrabenzonaphthalene (TBN) $\left(x_{\text {TBN BaP }} \leq 1\right.$ for polymeric $C_{18}: x_{\text {TBN BaP }}>1.7$ for monomeric $C_{18}$ phases) [21]. The selectivity of sixty-five columns was evaluated for a mixture of seven carotenoids. using methanol and acetonitrile or these solvents modified with tetrahydrofuran (THF) or ethyl acetate: recovery of the carotenoids from the columns was measured as well. In that study. several conclusions were drawn: under the conditions used. polymeric and some intermediate columns were able to separate lutein and zeaxanthin. but monomeric columns could not: methanol-based solvents provided higher carotenoid recoveries from the column than acetonitrilebased solvents; and if a modifier was used. THF provided slightly better recovery than ethyl acetate and less modifier was required [20].

\section{Column selection}

The narrow-pore Bakerbond $\mathrm{C}_{18}$ column that was selected for this method is prepared by a polymeric synthesis [22], but was classified as intermediate in the earlier study using the PAH test mixture [21]. A second column. while nominally the same as the column evaluated in the sixtyfive-column comparison study, was classified as

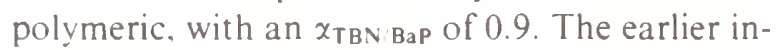
termediate column $\left(x_{\mathrm{TBN} / \mathrm{BaP}}=1.27\right)$ was one of the few intermediate columns able to resolve lutein and zeaxanthin in our column comparison study, but its recovery of the carotenoid mixture used in that study was low $(75 \%$ recovery using $100 \%$ methanol as the mobile phase, $66 \%$ recovery using 5\% THF-95\% acetonitrile). These recoveries. which were unacceptable, did not present a problem when recovery was improved by adding ammonium acetate and TEA to the mobile phase.

\section{Solvent selection}

Results of the column comparison study indicated that carotenoid recovery was better using THF rather than ethyl acetate and also better using methanol rather than acetonitrile. However, to achieve the desired separation with this col- umn, it was necessary to use an acetonitrile-based solvent despite the observations described above. We were unable to obtain an acceptable separation using various combinations of THF. methanol. and acetonitrile, and instead developed a method using ethyl acetate. methanol. and acetonitrile.

Acid contamination in chlorinated solvents has been blamed for carotenoid losses [23]: because hydrolysis of ethyl acetate produces acetic acid. acidity may have caused the losses observed when ethyl acetate was used in the column comparison study. Ammonium acetate. which was necessary as a modifier. is poorly soluble in ethyl acetate and in acetonitrile. The addition of 0.05 $M$ ammonium acetate to the methanol alone provided sulficient buffer capacity (assuming that acidity is the factor critical to recovery) so that recoveries were high even though ethyl acetate and acetonitrile were used. Although initial separations in an earlier iteration of the method were fine after the column was flushed with buffer. carotenoid recovery gradually decreased without the addition of ammonium acetate to the mobile phase itself. When a mixture of lutein. zeaxanthin. $\beta$-cryptoxanthin. lycopene. $\alpha$-carotene. $\beta$-carotene. and trans- $\beta$-apo- $8^{\prime}$-carotenal (an earlier internal standard) was chromatographed using the earlier method without the addition of ammonium acetate. recovery was only $75 \%$. Using the same separation procedure with the addition of ammonium acetate. recovery of that carotenoid mixture was $92 \%$.

Kamber and Pfander [24] found it necessary to add N.N-diisopropylethylamine to silica columns to obtain good carotenoid recovery. N.NDiisopropylethylamine is a nucleophilic base: in the absence of this compound. we examined TEA. TEA added to each of the three solvents (ace tonitrile. methanol containing $0.05 \mathrm{M}$ ammonium acetate. and ethyl acetate) improved recovery. Using the method described in this paper with $0.1 \%$ TEA added to each of the three solvents, carotenoid recovery was $101 \%$. When $0.1 \%$ TEA was added to the solvents. and the methanol did not contain ammonium acetate. recovery was only $89 \%$. TEA behaves as a strong 
modifier. and reduces retention times such that several peaks were no longer resolved when a TEA concentration of $0.1 \%$ was used. Thus. as a compromise. this method uses $0.05 \%$ TEA in each of the solvents and $0.05 .1 /$ ammonium acetate in the methanol: recovery is $94 \%$. and the necessary peaks are resolved.

Chromatograms for the analys of an extract of human serum using this method are shown in
Fig. 3: all analytes were naturally occurring except for retinyl palmitate. which was spiked into the serum. Resolution of lutein and zeaxanthin is superior to most published methods. $\beta$-cryptoxanthin is well resolved from lycopene and its isomers. and the cis trans isomers of $\beta$-carotene are resolved. although the $c$ is isomers are not base. line-resolved. The method's applicability to food separations is demonstrated in Fig. 4. In this fig-

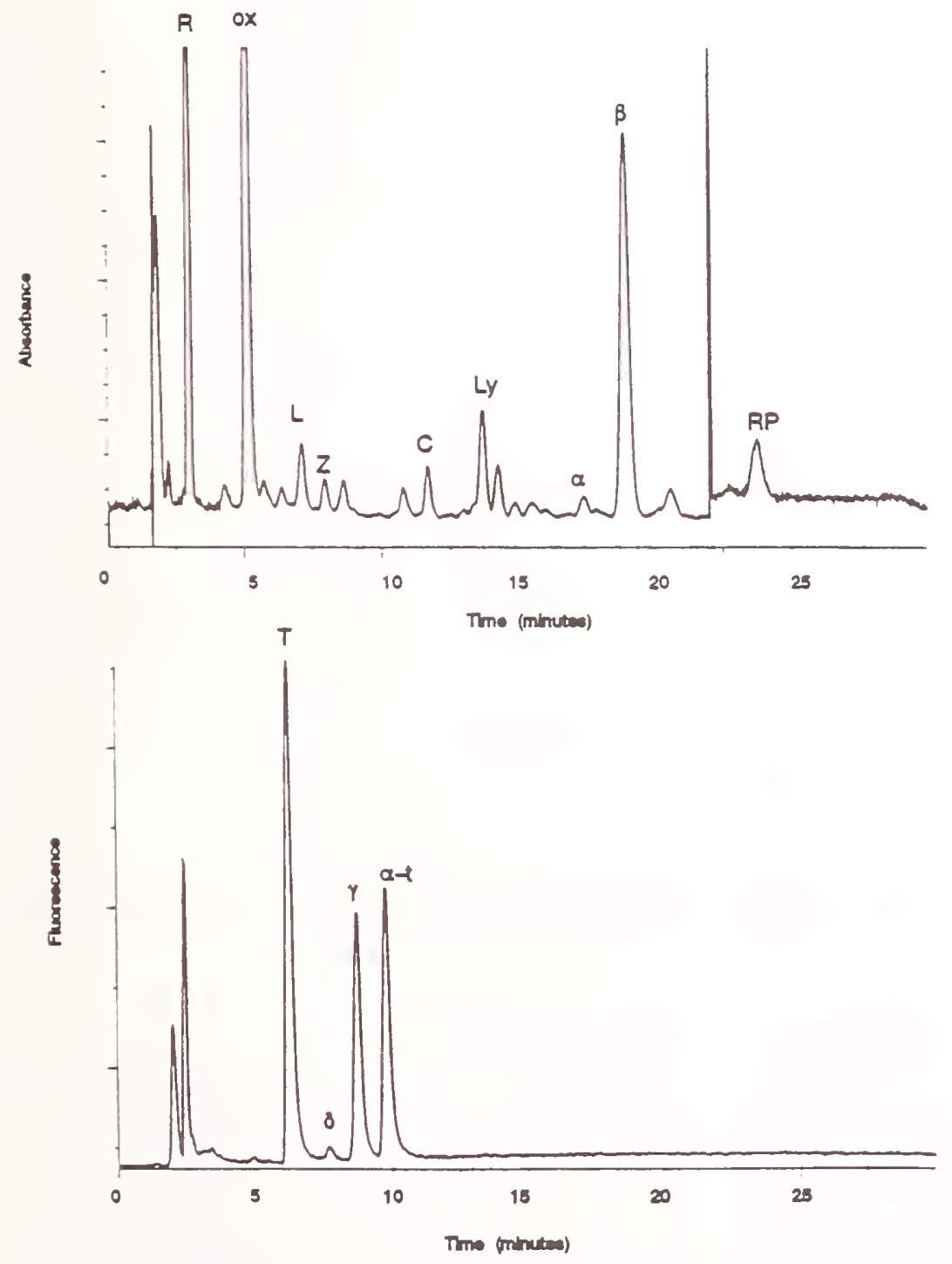

Pg. : Chromatogram from the reversed-phase LC separation of retinol (R. $0.734 \mu \mathrm{g} \mathrm{ml}$ ). truns- $\beta$-apo-10'-carotenal oxime (ox). lutein

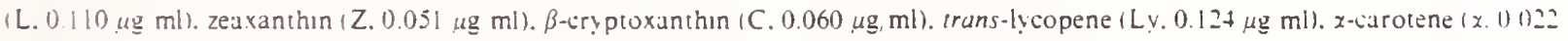
$\mu \mathrm{g} \mathrm{ml}$. (rans- $\beta$-carotene $(\beta, 0.567 \mu \mathrm{g} \mathrm{mi})$, and retınyl palmutate (RP. spiked. $0.297 \mu \mathrm{g} \mathrm{ml}$ ) in human serum usıng absurbance detection. Chromatugram of tocol (T). $j$-tocopherol $(\delta .0 .084 \mu \mathrm{g}, \mathrm{ml})$. $;$-tocopherol $(\%, 1.94 \mu \mathrm{g} \mathrm{ml})$. and $x$-tocopherol $(x-t, 5.69 \mu \mathrm{m}$ l) using fluorescence detection. 

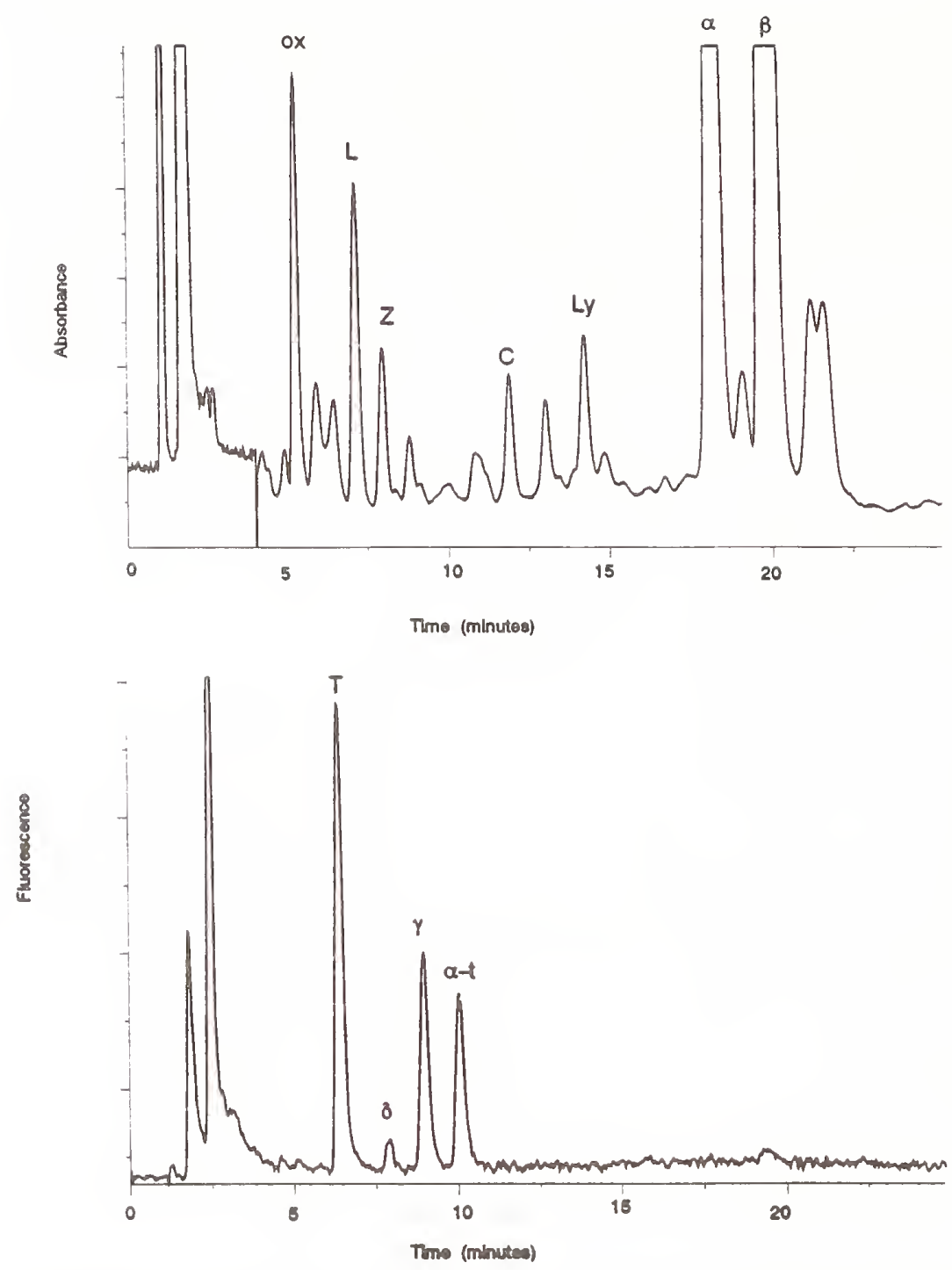

Fig. 4. Chromatogram from the reversed-phase LC separation of retinol. carotenoids, and tocopherols in a mixture of apricuts. carrots. creamed corn, creamed peas, green beans, orange juice. peaches. spinach. sweet potatoes, tomato paste, and winter squash. Labels are the same as those for Fig. 3.

ure the chromatograms from the analysis of an extract of the mixture of foods listed in the Experimental section contain all the major peaks that are present in serum as well as additional unidentified peaks. The resolution of the cis/trans isomers of $\alpha$-and $\beta$-carotene is more apparent in this chromatogram because of the increased levels of these isomers relative to those in the serum. (The cis isomers of $x$ - and $\beta$-carotene follow the trans compounds, which are labelled, in Figs. 3 and 4.)

\section{Internal standard selection}

During the analysis of serum samples other than the SRM, our first choice of internal standard for retinol and the carotenoids. trans- $\beta$ apo- 8 '-carotenal, co-eluted with polar carotenoids that were present in some of those samples. Thus, it was necessary to investigate different internal standards. trans- $\beta$-Apo- 10 '-carotenal eluted with an appropriate retention time, but only $65 \%$ of it was recovered from the column. Using an earlier method that did not employ TEA. the 
TABLE ॥

\section{RESLLTS OF THE ANALYSIS OF SRM 968. FAT-SOLLBLE VITAMINS IN HLIMAN SERUM}

Vean values were determined from the twetve values that resulted from analyss of two extracts from each of six bottles at each lewel Lincertantes (balues in parentheses) are one itandard desation from the mean. Lncertanties on certufied values represent $495 "$ " contidence intersul.

\begin{tabular}{|c|c|c|c|c|c|}
\hline Compound & Results $(\mu \cong \mathrm{m} \mid)$ & $\begin{array}{l}\text { Certutied relerence } \\
\text { values }(\text { th } \mathrm{m} /)\end{array}$ & Cumpound & Results ( $\mu \mathrm{g} \mathrm{ml})$ & $\begin{array}{l}\text { Cerutied reference } \\
\text { 'alues }(\mu \mathrm{g} \mathrm{ml})\end{array}$ \\
\hline \multicolumn{6}{|l|}{ Low lext } \\
\hline Retunul & $199(0.008)$ & $0.184 \pm 0.016$ & Irans-x-Carolene & $(0.1)+3:(0.00) 33$ & \\
\hline Lutein & $0.035(0.00121$ & 0.03 & Total $\beta$-carotene & $0 . x \leq 5(0) 056)$ & $0.4 \times 2=0.082$ \\
\hline Zeaxanthin & $0.013(0.001)$ & 0.02 & 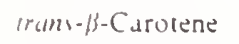 & 1) $=-0(0.0+x)$ & 0.9 \\
\hline$\beta-C r y p t o x a n t h ı n$ & $0.019(0.0021$ & 0.02 & Reuny! pulmilate & $\backslash D$ & \\
\hline Total lycopene & $0.160(0.019)$ & 0.2 & -Tocopherol & $19.11=10.030)$ & \\
\hline Iruns-Lycopene & $0.066(0) 0061$ & 0.07 & $\because$-Tucopherol & $2.66(0.05)$ & 28 \\
\hline Total x-curotene & $0.022(0.003)$ & $0.0 ?$ & $x$-Tucopherol & $10.36(0.25)$ & $10.40=026$ \\
\hline Irams-x-Carotene & $0.01 \geq 10.0011$ & & & & \\
\hline Total B-carotene & $0.231(0.01 \div)$ & 1) $264=0.074$ & High level & & \\
\hline 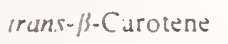 & $0.200(0.013)$ & 0.2 & Reunol & 0) $655(0.0191$ & $0.648=0.1126$ \\
\hline Retınyl palmitate & ND & & Luteın & $0.072(0.005)$ & 0.08 \\
\hline j-Tocopherol & $0.05+10.0111)$ & & Zeaxanthin & $0.027(0.002)$ & 0.03 \\
\hline$\because$ Tocupherol & $0.801(0.059)$ & 0.8 & B-Cryptoxanthın & $0.038(0.002)$ & 005 \\
\hline \multirow[t]{2}{*}{$x$-Tocopherol } & $+.61(0.38)$ & $1+1 \pm 0.22$ & Total lycopene & $0.140(0.022)$ & 0.4 \\
\hline & & & Iruns-Lycopene & $0.151(0.008)$ & 0.2 \\
\hline Medium level & & & Total $x$-carotene & $0.128(0.007)$ & 0.1 \\
\hline Retinol & $0 . \Sigma 16(0.013)$ & $0.495 \pm 0.021$ & Irans- $x$-Carotene & $0.080(0.006)$ & \\
\hline Lutein & $0.092(0.006)$ & 0.09 & Total $\beta$-carotene & $222(0.11)$ & $2.65 \pm 0.22$ \\
\hline Zeaxanthin & $0.037(0.003)$ & 0.04 & trans- $\beta$-Carotene & $1.9 \geq(0.10)$ & 2.4 \\
\hline$\beta$-Cryptoxanthin & $0.057(0.005)$ & 0.06 & Retınyl palmitate & $0.022(0.010)$ & \\
\hline Total lycopene & $0.46 !(0.022)$ & 0.4 & j-Tocopherol & $0.163(0.036)$ & \\
\hline Iruns-Lycopene & $0.179(0.011)$ & 0.2 & $\because$ Tocopherol & $3.70(0.09)$ & 3.9 \\
\hline Total $x$-ciarotene & $0.066(0.004)$ & 0.05 & $x$-Tocopherol & $16.04(0.59)$ & $16.19 \pm 0.36$ \\
\hline
\end{tabular}

$\checkmark N D=$ not detected: analyte not present or present at levels below the limit of detection.

addition of a small amount of formaldehyde $[500$ $\mu \mathrm{l}$ of a $30 \%$ (w, v) formaldehyde solution per $\mathrm{l}$ of solvent] to each of the three solvents improved recovery of trans- $\beta$-apo- $10^{\prime}$-carotenal ( $>90 \%$ recovery). But since recovery of this internal standard might vary over time, we converted the trans- $\beta$-apo- $10^{\prime}$-carotenal to trans- $\beta$-apo- $10^{\prime}$-carotenal oxime. which has approximately the same retention time as its aldehyde, is quite stable. and does not have a recovery problem. Thus, formaldehyde is no longer being added to the solvents. Although we did not observe loss of $\beta$-apo- $8^{\prime}$. carotenal in our earlier studies. it is probable that this aldehyde would be subject to the same losses as B-ilpo-10'-carotenal. For this reason the free carotenals are not recommended for use as internal standards.

\section{Analisis of serum samples}

Results for the analysis of SRM 968a are shown in Table II. Certified values are provided for retinol, total $\beta$-carotene. and $x$-tocopherol in SRM 968a: reference values are provided for lutein. zeaxanthin. $\beta$-cryptoxanthin. Irans-lycopene. total lycopene, $x$-carotene. trans- $\beta$-carotene, and $\because$-tocopherol. Certified values were determined by two NIST analysts using different methods and seven collaborating laboratories using different column-mobile phase combinitions. Reference values were determined by a lim. 
TABLE III

MEAN STANDARD DEVIATION, AND RELATIVE STANDARD DEVIATION FOR TWO QUALITY CON. TROL SERUM SAMPLES MEASURED FIFTEEN TIMES OVER A FIVE-WEEK PERIOD

\begin{tabular}{|c|c|c|c|}
\hline Compound & $\begin{array}{l}\text { Mean } \\
(\mu \mathrm{g} \mathrm{ml}\end{array}$ & $\begin{array}{l}\text { S.D. } \\
(\mu \mathrm{g} \mathrm{ml})\end{array}$ & $\begin{array}{l}\text { R.S.D. } \\
(\%)\end{array}$ \\
\hline \multicolumn{4}{|c|}{ Vormal quality control } \\
\hline Retinol & 0.474 & 0.010 & 2.1 \\
\hline Lutein & 0.117 & 0.005 & 4.0 \\
\hline Zeaxanthın & 0.039 & 0.002 & 4.9 \\
\hline$\beta$-Cryptoxanthin & 0.058 & 0.002 & 3.5 \\
\hline trans-Lycopene & 0.222 & 0.015 & 6.8 \\
\hline trans- $x$-Carotene & 0.037 & 0.001 & 3.5 \\
\hline trans- $\beta$-Carotene & 0.164 & 0.005 & 3.0 \\
\hline$\dot{\delta}$-Tocopherol & 0.076 & 0.012 & 15.2 \\
\hline$i$-Tocopherol & 1.93 & 0.07 & 3.7 \\
\hline$x$-Tocopherol & 8.58 & 0.32 & 3.8 \\
\hline \multicolumn{4}{|l|}{ Low quality control } \\
\hline Retinol & 0.366 & 0.011 & 3.1 \\
\hline Lutein & 0.091 & 0.004 & 4.9 \\
\hline Zeaxanthin & 0.030 & 0.002 & 7.5 \\
\hline$\beta$-Cryptoxanthin & 0.044 & 0.002 & 3.8 \\
\hline Irans-Lycopene & 0.167 & 0.006 & 3.8 \\
\hline trans- $x$-Carotene & 0.028 & 0.001 & 5.3 \\
\hline trans- $\beta$-Carotene & 0.128 & 0.004 & 3.2 \\
\hline$\dot{\delta}$-Tocopherol & 0.059 & 0.008 & 14.0 \\
\hline$\because$-Tocopherol & 1.49 & 0.04 & 2.4 \\
\hline$x$-Tocopherol & 6.64 & 0.21 & 3.2 \\
\hline
\end{tabular}

ited number of analyses and are provided by NIST for information only. (The method described in this paper was not used to assign values to the SRM.) Results show good agreement with reference and certified values for retinol, the tocopherols, and most of the carotenoids. Total $\beta$-carotene results obtained using this method are somewhat low for the medium- and high-level SRM when compared to the certified values, but are comparable to results obtained when the SRM was distributed to 45 laboratories involved in a round robin study. As reported in the SRM Certificate of Analysis, the medium- and highlevel total $\beta$-carotene concentrations determined in the round robin were $0.89 \pm 0.12$ and $2.34 \pm$ $0.34 \mathrm{mg} / 1$, respectively.

Results for the analysis of the two quality con- trol sera are shown in Table III. Means are provided to show the levels at which the given relative standard deviations (R.S.D.s) were attained. Most of the analytes had R.S.D.s in the 3-5\% range. The R.S.D. for $\dot{\delta}$-tocopherol is significantly higher due to the very low concentrations present in the serum (about two orders of magnitude lower than $x$-tocopherol). The R.S.D. for the low-concentration zeaxanthin is also somewhat high, probably because of the relatively low concentration in this sample.

\section{Dissolution of extract}

Dissolution of serum extracts is a critical step in the quantitative analysis of carotenoids in serum. The carotenoid concentrations are frequently near the detection limit. The carotenoids and lipid residue are poorly soluble in polar organic solvents, yet the extract must be completely dissolved in a solvent miscible with the mobile phase and an adequate volume of the solvent injected without creating chromatographic artifacts. Dissolution can be facilitated by using ultrasonic agitation. Ideally the extract should be dissolved in the initial mobile phase or a slightly weaker solvent to focus the sample at the head of the column. We compared the effects on quantitation of dissolution in different solvents. After following the usual hexane extraction procedure. dissolution of the dried extract was compared in ethanol and in several other solvents. When 15:85 acetone-acetonitrile, methanol, a 20:80 methylene chloride-methanol, and 20:80 methylene chloride-acetonitrile were added to the dried extract, precipitates formed, and low recoveries were observed. Ethyl acetate dissolved the extract with no difference in recovery when compared to ethanol. Because of the increased solubility of lipids in ethyl acetate, we are currently reconstituting extracts in 50:50 ethyl acetate-ethanol. with $30 \mu \mathrm{g} / \mathrm{ml} \mathrm{BHT}$. It is important to note that peak broadening may become a problem when more than $20 \mu \mathrm{l}$ are injected into the predominantly acetonitrile mobile phase.

\section{Residual acid and metal activity}

The improved recovery resulting from the use 
of ammonium acetate and TEA is not limited to the analytical column used in the method described here. During the development of this method. we observed that exposure to minute quantities of trifluoroacetic acid caused the column to become unsuitable for carotenoid separations: previous carotenoid separations on the column were acceptable. but no peaks were obtained after the column had been exposed to traces of trifluoroacetic acid. Treatment with $50: 50$ methanol-0.01 . 11 ammonium acetate for $45 \mathrm{~min}$ restored the column to its initial carotenoid-separating state. The column could then be converted back and forth from "bad" to "good" at will by injecting dilute trifluoroacetic acid and washing the column with solvent containing ammonium acetate.

Following this observation. five columns that had provided poor recovery in our previous sixty-five-column evaluation were flushed with 0.05 $M$ ammonium acetate in methanol at $1 \mathrm{ml}$ min for $15 \mathrm{~min}$. then equilibrated for $15 \mathrm{~min}$ with THF-acetonitrile eluent pumped at $1 \mathrm{ml}$ min. After this buffer treatment. recovery improved on all five columns. Some columns showed greater improvement than others. Table IV shows the initial recoveries and recoveries following the buffer treatment. Mean improvement in recovery

TABLE IV

RECOVERY OF A CAROTENOID MIXTURE ON SE. LECTED COLUMNS BEFORE AND AFTER TREAT. MENT WITH 0.05 . 4 AMMONIUM ACETATE IN METHANOL WITH AND WITHOUT THE ADDITION OF $0.05 \%$ TEA TO AN ACETONITRILE-BASED MOBILE PHASE

Column Recovery (\%)

Betore treatment After treatment: Alter treatment: no TEA $\quad 0.05 \%$ TE.A

in mobile phase

\begin{tabular}{rrrr}
\hline 1 & 48 & 74 & 102 \\
2 & 1 & 30 & 99 \\
3 & 5 & 87 & 106 \\
1 & 15 & 69 & 104 \\
5 & 3 & 12 & $>87$ \\
\hline
\end{tabular}

across this group of five columns was $40^{\circ} \circ$. Thus. other recoveries in the earlier study may have improved if the columns had first been Hushed with an ammonium acetate buffer.

After observing that TEA further improved recovery. these same tive columns were again flushed for $15 \mathrm{~min}$ with $0.05 \mathrm{M}$ ammonium acetate in methanol. Columns were equilibrated for $15 \mathrm{~min}$ with $5.95 \mathrm{THF}$-acetonitrile containing $0.05 \%$ TEA. and carotenoid recovery was measured using this same solvent mixture. Results of this study are also included in Table IV. Recorery on all five columns improved dramatically from initial recoveries. Recovery for the fifth column is " $>87^{\circ}, \cdots$ because not all the pealks had eluted during the 25 -min run time using the mobile phase composed of 15:85 THF-acetonitrile containing $0.05 \%$ TEA. Reproducibility of recovery measurements in the column comparison study was $\pm 6 \%$ : recoveries in Table IV that are greater than $100 \%$ are within the limits of the method.

These same five columns that had provided poor recovery in the sixty-five-column study were also tested for trace metal activity. Acetylacetone is used as an indicator of metal activity: the sharper the acetylacetone peak. the less metal ictivity present [25]. Of the five columns tested. the one with the highest initial recovery had the least metal activity. Columns $t$ and 5 . two columns from the same manufacturer. exhibited high metal activity. Columns 2 and 3. also from one manufacturer. exhibited similar metal activity. but less than columns 4 and 5 . Although adding TEA to the mobile phase improved recovery on these five columns. it is not likely that TE.A is functioning as a chelating agent. However. because of its nucleophilic character. TEA mav interact with positively charged metal species that may be present in the silica. The use of nucleophilic bases has been previously reported to improve carotenoid recovery on normal-phase LC columns [24,26]. Liew et al. [27], in a study of the role of metal ions in bleaching clays. found that $\mathrm{Fe}^{3-}$. $\mathrm{Mg}^{2+}$, and $\mathrm{Ca}^{2-}$ play a significant role in the clay's ability to adsorb $\beta$-carotene. They suggested that the $\beta$-carotene is adsorbed directly to the 
ferric ion. in particular, and then undergoes further reaction [27]. At the same time. they believe that the presence of metal ions causes the dissociation of water to form metal hydroxides and protons. which also cause carotenoid degradation [27]. While there is very little water in our system $1<1 \%$ from the ammonium hydroxide and the acetic acid used to prepare the ammonium acetate). the improvement of carotenoid recovery with the addition of ammonium acetate (a buffer) and TEA (a base) in our study supports their conclusions.

\section{CONCLUSIONS}

In this paper. an LC method is described for the measurement of carotenoids. retinol. retinyl palmitate, and the tocopherols in serum and in food. Retinol, retinyl palmitate. and the carotenoids are separated and measured using a programmable UV-Vis detector. The tocopherols are measured simultaneously using a fluorescence detector in series. Apo-carotenals used as internal standards in our initial methods are not acceptable for use in the analysis of all serum samples because of variable recovery and co-elution with serum carotenoids that are more polar than lutein. Because of this. we have examined several other internal standards, and have selected trans$\beta$-apo-10'-carotenal oxime.

Using several different columns, we have also demonstrated that by flushing with a mixture of ammonium acetate and methanol, columns that provide little or no recovery of carotenoids can be improved. TEA added to the solvents also improves carotenoid recovery. The ammonium acetate and the TEA appear to be performing two different functions, since the addition of one without the other did not improve recovery as much as the addition of both in studies with the analytical column used with this method.

\section{ACKNOWLEDGEMENTS}

This work was supported in part by the $\mathrm{Na}$ tional Cancer Institute, Division of Cancer Etiology. Agreement No. Y01-CP9-0513. We thank
Lane Sander (NIST) for valuable insights regarding recovery problems, Garry Handelman (University of California at Davis) for providing carotenoid compounds to test as internal standards. Willy Schüep of Hoffman-LaRoche for providing trans- $\beta$-apo- $10^{\prime}$-carotenal for the synthesis of the trans- $\beta$-apo- $10^{\prime}$-carotenal oxime internal standard, and Shizumasa Kijima of Esai for providing tocol for use as an internal standard.

\section{REFERENCES}

1 R G. Ziegler. .4m. J. Clin. Vurr.. $53(1991) 251 \mathrm{~S}$.

2 J. G. Bierı. E. D. Brown and J. C. Smith. Jr.. J. Liq. Chromalogr.. $8(1985) 473$

3 H. J. C. F. Nelis and A P. De Leenheer. Anul. Chem.. 55 (1983) 270

4 M. Stacewicz-Sapuntzakis. P. E. Bowen. J. W. Kikendall and M. Burgess. J. .Hicronutr. Anal.. 3 (1987) 27.

5 R. Ohmacht. Gy. Toth and G. Voigt. J. Chromutogr.. 395 (1987) 609

6 C. S. Yang and M.-J. Lee. J. Vutr Grow sh Cancer. $+119 \times 7)$ 19.

7 G. Cavina. B Gallinella. R. Porra. P. Pecora and C. Suracl. J. Pharm. Biomed. Anal. 6 (1988) 259

8 A. B. Barua. R. O. Batres. H. C. Furr and J. A. Olson. $J$. .hicronutr. Anul. 5 (1989) 291.

9 L. R. Cantilena and D. W. Nierenberg. J. Micromutr. Anul.. 6 (1989) 127

10 W. A. MacCrehan and E. Schönberger. Clin. Ch'm.. 33 (1987) 1585.

11 A. L. Sowell. D. L. Huff. E. W. Gunter and W. J. Driskell. J. Chromatogr.. 431 (1988) 424

12 B. Olmedilla. F. Granado. E. Rojas-Hidalgo and I. Blanco. J. Liq. Chromatogr.. 13 (1990) 1455

13 W. G. Rushin. G. L. Catignanı and S. J. Schwartz. Clin. Chem.. $36(1990) 1986$.

14 N. E. Craft. L. C. Sander and H. F. Pierson. J. Micromur. Anal.. $8(1990) 209$

15 E. Lesellier. C. Marty. C. Berset and A. Tchapla. J. High Resolut. Chromatogr.. 12 (1989) $\$ 47$.

16 A. Pettersson and L. Jonsson. J. Micromur. Anul.. \& (1990) 23.

17 D. Hess. H. E. Keller. B. Oberlin. R. Bonfanti and W Schüep. Int. J. V'it. Vutr. Res.. 61 (1991) 232

18 G. W. T. Groenendijk. W. J. De Grip and F. J. M Duemen. Biochim. Biophis. Acta. $617(1980)+30$.

19 L. C. Sander and N. E. Craft. Anal. Chem.. 6? (1990) 1545

20 K. S. Epler. L. C. Sander. R. G. Ziegler. S. A. Wise and .V. E. Craft. J. Chromatogr.. 595 (1992) 89.

21 L. C. Sander and S. A. Wise. LC. GC. 5 (1990) 378.

22 M. Henry. J. T. Baker. Phillipsburg. NJ. personal communıcation.

23 G. Brition. Methods En=lmol.. 111 (1985) 113. 
24 . M. Kamber and H. Pfander. J. Chromulogr.. 295 (1984) 295 25 M. Verzele and C. Dewaele. Chromarographia. 18 (1984) 84

26 J. K. Abaychı and J. P. Riley. Anul. Chim. Acra. 107 (1979) 1.
27 K. Y. Liew. S. H. Tan. F. Morsingh and L. E. Khors. J. Am. Oil Chem. Soc.. 59 (1982) 480 . 


\title{
Determination of Retinol, $\alpha$-Tocopherol, and $\beta$-Carotene in Serum by Liquid Chromatography with Absorbance and Electrochemical Detection
}

\author{
William A. MacCrehan ${ }^{1}$ and Emil Schל̈nberger ${ }^{2}$
}

We describe a method for the determination of retinol, $\alpha$ tocopherol, and $\beta$-carotene in serum, using a liquid-chromatographic separation with wavelength-programmed ultraviolet/visible absorbance and amperometric electrochemical detection with a glassy carbon electrode. After protein denaturation and addition of an internal standard, tocol, $250-\mu \mathrm{L}$ samples are twice extracted with hexane. The reversedphase, gradient-elution chromatographic separation provides baseline resolution of: the all-trans isomer of retinol from the cis isomers, $\alpha$-from $\gamma$-tocopherol, and all-trans- $\beta$-carotene from $\alpha$-carotene and from cis- $\beta$-carotene isomers. The linearity of response and the detection limits for the two detectors for the three analytes are measured. A comparison of the values obtained for serum extracts shows good agreement between the absorbance and electrochemical detectors.

Additional Keyphrases: micronutrients - chromatography, reversed-phase - cis- $\beta$-carotene

In a quality-assurance role, the National Bureau of Standards (NBS) is supporting clinical research funded by the National Cancer Institute into the cancer-chemopreventive properties of vitamin A (all-trans-retinol), vitamin E ( $\alpha$ tocopherol), and pro-vitamin A (all-trans- $\beta$-carotene). ${ }^{3}$ The quality-assurance program is for laboratories involved in epidemiological studies, assaying these micronutrients in serum to evaluate the nutritional status of treated and control populations. For NBS analyses, we have developed a method to assay the three analytes by using liquid chromatography with ultraviolet/visible absorbance detection (LCUV) and oxidative electrochemical detection (LCEC), a new approach for the determination of all three micronutrients in serum.

In the past, all three analytes were determined by extraction followed by colorimetry $(1-4)$. That approach is subject to nonspecific interference from other matrix constituents, such as carotenoid and xanthophylls as well as unresolved

\footnotetext{
${ }^{1}$ National Bureau of Standards, Center for Analytical Chemistry, Organic Analytical Research Division, Gaithersburg, MD 20899.

${ }^{2}$ National Physical Laboratory, Danzinger Bldg "A," Hebrew University, Givat Ram, Jerusalem, Israel 91904.

${ }^{3}$ Nonstandard abbreviations: NBS, National Bureau of Standards; LCUV, liquid chromatography with ultraviolet/visible absorbance detection; LCEC, liquid chromatography with electrochemical detection; BHT, 2,6-t-butyl-4-methylphenol.

Received March 2, 1987; accepted June 17, 1987.
}

isomers of the analytes. With the advent of liquid-chromatographic separations, unrecognized interferences present in the earlier techniques became apparent, and those procedures are no longer considered reliable.

Many liquid-chromatographic methods have been proposed for the single $(5-11)$ as well as the simultaneous multiple determination (12-21) of the three micronutrients in serum. Fixed-wavelength ultraviolet/visible absorbance detection can be used to measure the analytes at their expected serum concentrations, but this approach relies heavily on the degree of chromatographic resolution to provide bias-free results. Fluorescence detection $(20,21)$ has been used to enhance both selectivity and sensitivity of assays for retinol and tocopherol. Oxidative electrochemical detection has also been used for the determination of tocopherol $(9,19,22)$, but its use for detecting retinol and carotene has only been suggested (22). The electro-oxidation of retinol $(23,24)$ and $\beta$-carotene $(25)$ has been studied only in nonaqueous solvents. Recently, we have studied the oxidation of retinol at a glassy carbon electrode in methanol/ water solvent such as those used in LC separations (26). The oxidation is a multiple-electron process that forms several products. The approach has been found suitable for the thin-layer amperometric detection of chromatographically separated all-trans as well as the cis isomers of retinol (26).

The objectives of this work were (a) to evaluate the extraction procedure used to recover the analytes from sera, (b) to develop a reversed-phase separation that would provide baseline resolution of the analytes of interest from other serum constituents and from their isomers, and $(c)$ to investigate the utility of oxidative amperometric detection for the simultaneous determination of retinol, $\alpha$-tocopherol, and $\beta$-carotene.

\section{Materials and Methods}

\section{Reagents/Standards}

Standard solutions of the three analytes and the internal standard were prepared by dissolving crystals (all-transretinol, all-trans- $\beta$-carotene) or oils ( $\alpha$-tocopherol, tocol), in absolute ethanol; the concentrations were determined spectrophotometrically, corrected for impurities that absorb at

\footnotetext{
${ }^{4}$ Certain commercial equipment, instruments, or materials are identified in this paper in order to specify adequately the experimental procedures. Such identification does not imply recommendation or endorsement by the National Bureau of Standards, nor does it imply that the materials or equipment identified are necessarily the best available for the purpose.
} 
the wavelength of measurement (see below). The tocol internal standard was added in equal concentrations to standard mixtures and the serum samples before extraction. All-trans-retinol (type $\mathrm{N}$ ), retinyl acetate (type I), retinyl palmitate (type IV), all-trans- $\beta$-carotene (type IV), and alltrans- $\alpha$-carotene (type $V$ ) were obtained from Sigma Chemical Co., St. Louis, MO. dl-Tocol was a gift from Hoffmann-La Roche, Basel, Switzerland. $d$ - $\alpha$-Tocopherol ("99\% minimum" grade), $d$ - $\gamma$-tocopherol, and $d$ - $\alpha$-tocopherol acetate came from Eastman Kodak, Rochester, NY. 2,6-Di-t-butyl-4methylphenol (BHT) was obtained from Calbiochem, La Jolla, CA. Lutein, zeaxanthin, cryptoxanthin, and nonaprene were a gift (G. R. Beecher, USDA, Beltsville, MD). All were stored at $-4{ }^{\circ} \mathrm{C}$ upon receipt and thawed just before preparation of ethanol solutions.

The concentrations of the three analyte standards were determined with a double-beam grating spectrophotometer vs an absolute ethanol reference. The absorptivity values ( $\mathrm{L}$ $\mathrm{cm}$ ) in ethanol that we chose for the calibration are: alltrans-retinol $\epsilon_{324 \mathrm{~nm}}=1850(27), \alpha$-tocopherol $\epsilon_{292 \mathrm{~nm}}=75.5$ (13), and all-trans- $\beta$-carotene $\epsilon_{452} \mathrm{~nm}=2620$ (28). The solutions were diluted such that the absorbance of the solution was always between 0.1 and $0.9 \mathrm{~A}$. Once prepared, the purity of the individual standard solutions was evaluated by the "slow-gradient" LCUV separation described below. If the total impurity at $\lambda_{\max }$ was between $1 \%$ and $5 \%$, as determined by the total area under all chromatographic peaks (other than that for the desired analyte), then the absorbance of the solution was corrected for the impurities; otherwise, the solution was discarded and fresh material was taken for measurement. From the individual solutions, we prepared dilute standards in ethanol, adding BHT (20 $\mathrm{mg} / \mathrm{L}$ ) as a preservative (5). The approximate final concentrations in our working standards for the analyte mixture (in $\mathrm{mg} / \mathrm{L}$ ) were: all-trans-retinol 0.5 , tocol $7, \alpha$-tocopherol 7 , and all-trans- $\beta$-carotene 0.2 . Aliquots of this mixture were stored in glass vials at $-70^{\circ} \mathrm{C}$ and were stable for at least one month. Before use, we warmed the solutions to about $40^{\circ} \mathrm{C}$, agitated them ultrasonically, then cooled them to room temperature.

\section{LC Apparatus}

For the simultaneous determination of the three analytes, we used a two-pump, computer-controlled solvent-delivery system. Each pump had two reciprocating pistons with pressure-feedback compensation. The automated sample injector was used with Teflon-septa-sealed vials. To separate the analytes and their isomers, we used a $0.46 \times 25 \mathrm{~cm}$ Vydac (The Separations Group, Hesperia, CA) octadecylsilyl-modified silica $\left(\mathrm{C}_{18}\right)$ column (201TP) packed with $5-\mu \mathrm{m}$ particles. This column has a polymeric bonded phase with large $(30-\mathrm{nm})$ pores, and can separate all of the retinol isomers in $30 \mathrm{~min}$ (26). For absorbance detection we used a wavelength-programmable detector (Model 783; Kratos Analytical, Ramsey, NJ) with a noise specification of $1 \times 10^{-5}$ $A$ (1-s time constant, $236 \mathrm{~nm}$ ), and a deuterium lamp. The electrochemical detector was a thin-layer amperometric detector cell (Model TL-6A; Bioanalytical Systems, West Lafayette, IN) modified by replacing the original working electrode block with a custom Teflon holder and a $1.2 \mathrm{~mm}$ diameter glassy carbon (Fluorocarbon Co., Anaheim, CA) rod. The filling solution for the silver/silver chloride reference electrode contained methanol/water ( $70 / 30$ by vol before mixing) and $0.1 \mathrm{~mol}$ of $\mathrm{KCl}$ per liter. All potentials are specified with respect to this reference electrode. We used this reference solution to help address the problems in the commercial electrode of the widely disparate internal (3 $\mathrm{mol} / \mathrm{L}$ aqueous $\mathrm{KCl}$ solution) and external (methanol/water mobile phase) electrolytes. A 2-s time constant was used for both detectors.

\section{Separation Conditions}

Distilled-in-glass solvents (B \& J Brand; American Scientific Products, McGaw Park, IL) were used in preparing the mobile phase. The electrolyte we used was a combination of Suprapur-grade ammonium hydroxide and acetic acid (Merck, Darmstadt, F.R.G.), which provided a buffer of $\mathrm{pH}$ 3.5. This electrolyte was included in all mobile-phase mixtures at a final concentration of $0.02 \mathrm{~mol} / \mathrm{L}$. The electrolyte not only was necessary to conduct electrical current for the amperometric detector, but also enhanced the reversedphase separation of the analytes from their isomers. For simultaneous determination of the three analytes, we used the following "slow-gradient" solvent program: initial solvent, water/methanol $n$-butanol (15/75/10, by vol); final solvent, water/methanol $n$-butanol ( $2 / 88 / 10$ by vol). The initial solvent composition was used for $3 \mathrm{~min}$, then was changed linearly to the final solvent composition over a 15-min period, and was held at the final composition for $17 \mathrm{~min}$ before being returned to the initial solvent composition. The flow rate was constant at $1.5 \mathrm{~mL} / \mathrm{min}$. The "fast-isocratic" mobile phase was water/methanol $n$-butanol (2.6/87.4/10 by vol), with an initial flow rate of $1.5 \mathrm{~mL} / \mathrm{min}$; the flow rate was increased to $3.0 \mathrm{~mL} / \mathrm{min}$ after $10 \mathrm{~min}$, held constant for $5 \mathrm{~min}$, then returned to $1.5 \mathrm{~mL} / \mathrm{min}$.

\section{Sample Preparation}

We used three steps to extract the micronutrients from serum for LC analysis: protein denaturation, organic solvent extraction, and solvent exchange. Frozen sera, stored in glass ampules at $-70^{\circ} \mathrm{C}$, were thawed thoroughly to room temperature, and vortex-mixed for $5 \mathrm{~s}$ before sample aliquots were removed. We placed $250 \mu \mathrm{L}$ of serum in a 13 $\mathrm{mL}$ centrifuge tube, added $25 \mu \mathrm{L}$ of an ethanolic solution of the internal standard, tocol, and vortex-mixed briefly. We then denatured the proteins with $250 \mu \mathrm{L}$ of ethanol containing BHT, $15 \mathrm{mg} / \mathrm{L}$. After vortex-mixing the sample for $1 \mathrm{~min}$ with $1.5 \mathrm{~mL}$ of hexane extractant, we centrifuged $(\approx 2000 \times$ $g$ ) it for about $2 \mathrm{~min}$, then removed $1.0 \mathrm{~mL}$ of the upper layer. We added a fresh $0.5 \mathrm{~mL}$ of hexane to the remaining lower layer, vortex-mixed for $1 \mathrm{~min}$, then centrifuged $2 \mathrm{~min}$. We collected $300 \mu \mathrm{L}$ of the second extract and combined it with the first extract. To remove the hexane, we placed the sample in a container with a two-holed polyethylene lid and blew dry nitrogen gas over it, to avoid oxidation by atmospheric oxygen. Finally we dissolved the residue in $250 \mu \mathrm{L}$ of ethanol containing BHT, agitating the sample ultrasonically for $1 \mathrm{~min}$. Occasionally, the redissolved extract was cloudy, and an additional centrifugation was necessary before LC analysis. We placed the redissolved extract in a glass sample vial with an air-tight Teflon-lined septum, and injected $25 \mu \mathrm{L}$ into the chromatograph.

\section{Results and Discussion}

\section{Extraction of the Analytes from Serum}

Although retinol, tocopherol, and carotene are highly insoluble in water, they are soluble in serum because they are transported by lipoproteins. The proteins involved may be $80 \%$ to $90 \%$ lipid enclosed in a hydrophilic protein sheath. 
Specific retinol-binding-proteins are known to selectively transport retinol in vivo. The presence of lipoprotein encapsulation imposes several constraints on the available approaches to the determination of these micronutrients in serum. First, it makes impossible meaningful recovery experiments for these analytes in the serum matrix based on a simple addition of the free analyte dissolved in solution. As noted by Bieri et al. (7), the procedure of addition of analyte solution to the serum prior to extraction fails to adequately simulate the naturally encapsulated analytes because of the slow kinetics of uptake by the lipoproteins. The slow kinetics of the analyte transfer to and from the lipoprotein encapsulation is demonstrated by the failure of hexane to directly extract the analytes without the addition of protein denaturant. In such an experiment we found that tocopherol is only partially extracted, with little recovery of retinol and carotene. Although this standard addition approach has been used to determine extraction efficiencies in many studies $(12,14-18,20-22)$, it may provide unrealistic "recovery" results for these analytes.

To overcome the serum lipoprotein-binding of the analytes for solvent extraction, one must first denature the proteins, then use a water-immiscible organic solvent to extract the unencapsulated analytes. The final aspect of the lipoprotein-binding of the serum micronutrients that must be resolved before complete recovery is possible is the requirement of effective competition of the extraction solvent with the serum lipids for solvation of the analytes. Hexane has been most frequently used for this purpose, with a large ratio of the volume of organic extractant to serum providing suitable competition to overcome lipid-binding. However, even if the distribution ratio of the analytes into the hexane from the precipitated serum were infinite, the recovery would still be $<100 \%$, owing to the volume of hexane (about $20 \%$ in our method) that does not separate into the upper layer, presumably because it is bound in lipid micelles in the aqueous lower layer. The unrecovered volume of extractant graphically demonstrates the importance of the serum lipids in the procedure and points to the advisability of the addition of a suitable internal standard to correct for the volume of extractant not recovered.

We chose to use tocol (29) as internal standard to correct for the variations in extractant volume. Tocol is structurally very similar to $\alpha$-tocopherol but lacks the three methyl groups on the phenolic ring. It is eluted in an uncrowded portion of the chromatogram and responded to both absorbance and electrochemical detectors. We also examined the use of three alternative internal standards: two esters of the vitamins-retinyl acetate $(12,18,20)$ and $\alpha$-tocopherol acetate $(7,12)$-and a $\beta$-carotene analog, nonaprene $(30,31)$. Retinyl acetate was unstable and eluted, with two shoulders, on the LCUV chromatogram. $\alpha$-Tocopherol acetate was not suitable as internal standard for LCEC, because the esterification of the electro-active phenolic hydroxy group of the tocopherol ring increased the applied potential required to $+1100 \mathrm{mV}$, where detector selectivity and sensitivity are poor. Nonaprene, a promising internal standard for carotenoid analysis $(30,31)$, is an analog of $\beta$-carotene having an additional isoprene unit; however, in our method, nonaprene co-eluted with serum components that were retained longer than $\beta$-carotene, making it unsuitable as an internal standard.

We evaluated the effect of different protein denaturation solvents on analyte recovery. Acetone, acetonitrile, methanol, and perchloric acid (100 $\mathrm{mL} / \mathrm{L})$, in equivolume ratio to the serum sample, yielded less recovery of all three analytes than ethanol did.

Because of the loss of extractant volume, we examined the utility of a second extraction of the serum denatured with fresh solvent. We used a pooled serum sample and measured the analytical recovery of the added internal standard, tocol. After a single extraction, recovery was 81.0 (SD 15.2)\% $(\mathrm{n}=$ 9). Combining a second extraction with the first recovered 96.8 (SD 3.0)\% of the added tocol ( $n=72$ ). The recovery of the three analytes was also improved by including the second extraction. Although this additional extraction adds to the complexity of the procedure, it is necessary for assay precision.

We evaluated alkaline hydrolysis of the serum prior to the extraction, as outlined by Thompson et al. (8), for the determination of micronutrients in serum, comparing the results with those obtained in our method. Results were identical, within statistical error, for $\beta$-carotene. For alltrans-retinol, however, the alkaline extration method provided higher results than our method did, because of the additional free retinol recovered by hydrolysis of serum retinol esters. No $\alpha$-tocopherol was recovered from the alkaline hydrolysate because of conversion to the hydrophilic phenolate anion.

We also tested a standardization approach based on the addition of increments of an ethanol solution of the three analytes to bovine serum albumin, followed by extraction $(15,16)$. The rationale for this approach is that this albumin is commercially available, contains none of the three micronutrients, and provides a matrix similar to human serum. Although matrix-matching for standardization often has merit, we found serious bias with this particular method: only about $70 \%$ of the added all-trans-retinol and $\alpha$-tocopherol and about $40 \%$ of $\beta$-carotene were recovered from the bovine serum albumin matrix. Because of the low recovery of the standards in this matrix, the final determined results for the three micronutrients in test human serum were quite high. Thus, this method is not recommended (32).

\section{Separation of the Analytes}

Some methods for the determination of all three micronutrients involve an isocratic separation $(15,16,18)$. However, the advantage of rapid repetitive analyses is offset by the significant compromises that must be made in the selectivity of the chromatographic separation for all-trans-retinol and $\alpha$-tocopherol. Because the reversed-phase retention of these two analytes is much less than for $\beta$-carotene, baseline resolution of the micronutrients from their isomers and other serum components in a short isocratic chromatographic procedure is not possible with current column technology. Two types of analytical compromises are illustrated when a "rapid isocratic" separation is used (Figure 1): (a) peak area integration of the three analytes is made less precise by the presence of partly co-eluting "shoulders," and (b) the accuracy is lowered by exactly co-eluting components, which are revealed only in long-gradient separations. Despite the long elution and recycle time required for gradient separations, we could not tolerate the attendant losses in the precision and accuracy resulting from the use of this fast-isocratic separation.

Thus, we used slow-gradient separation (Figure 2), which provides significantly better resolution of the three micronutrients from their isomers than shown in previously published reversed-phase analyses $(6,7)$. We chose this widepore, polymeric, octadecyl stationary phase in conjunction 

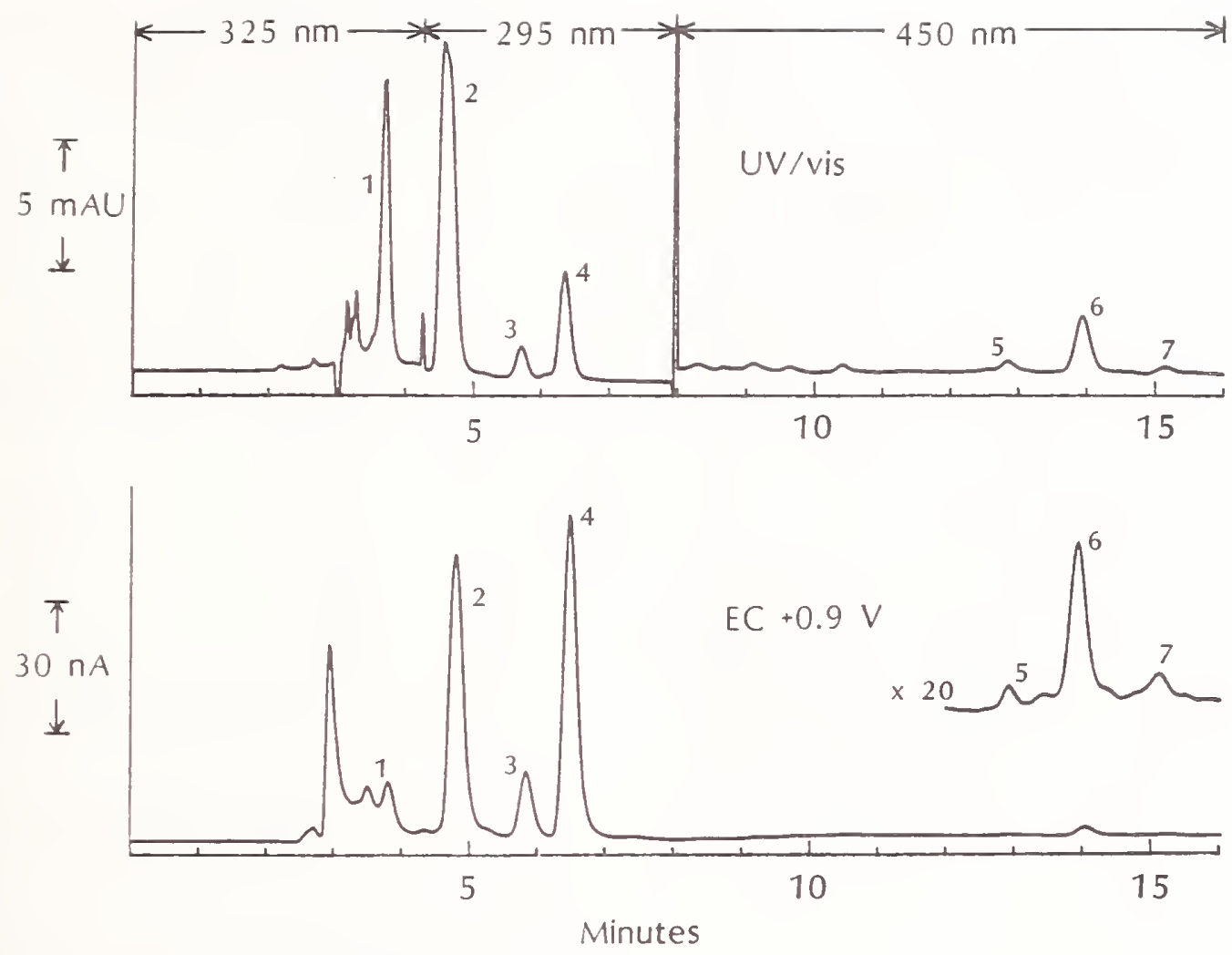

Fig. 1. Fast-isocratic separation of the micronutrients in a serum extract

Peaks: 1, retinol: 2, tocol; 3, rtocopherol; 4, a-tocopherol; 5, ar-carotene; 6, all-trans- $\beta$-carotene; 7, cis- $\beta$-carotene
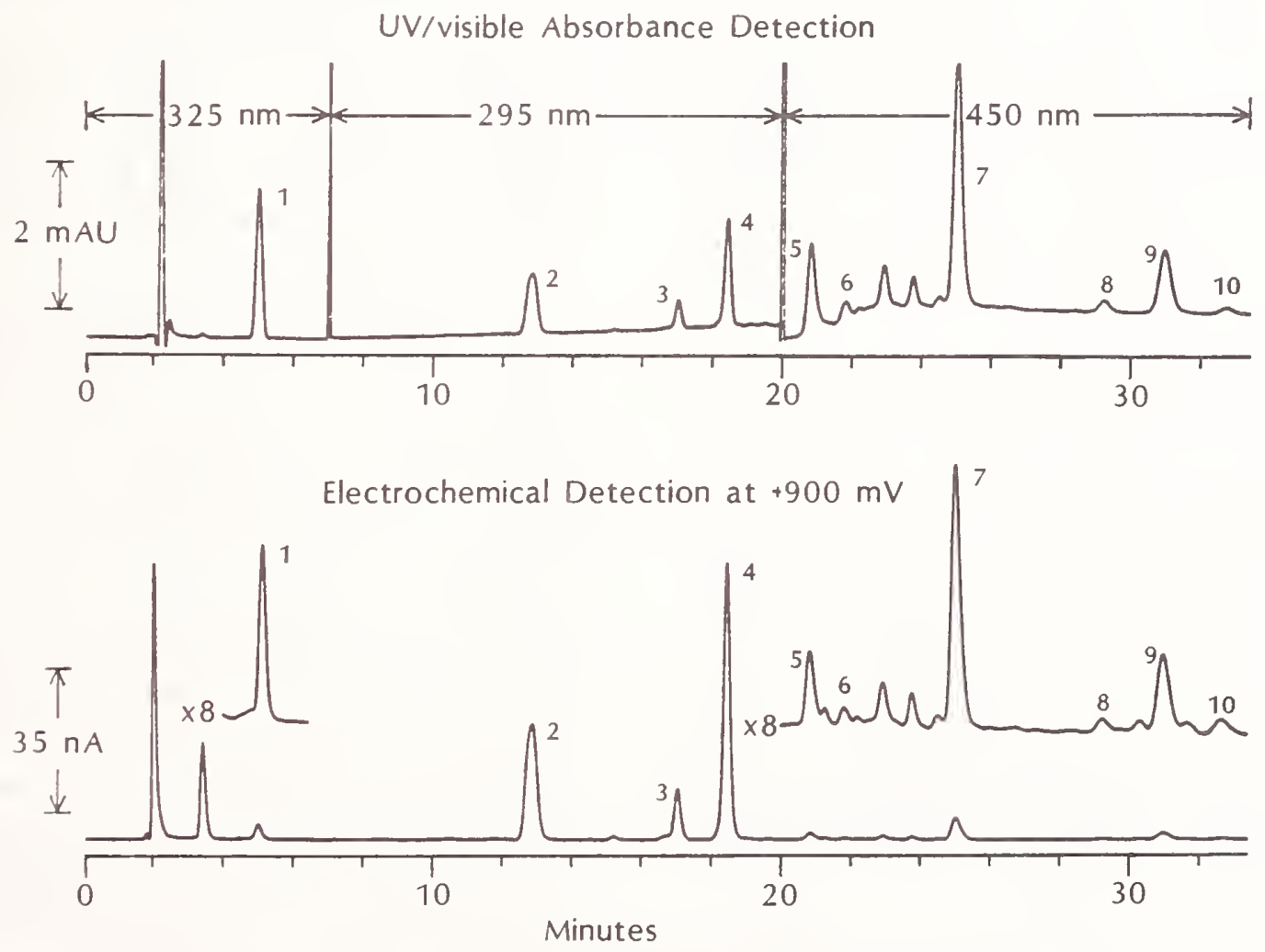

Fig. 2. Slow-gradient-elution separation of micronutrients in a serum extract

Peaks: 1, all-trans-retinol; 2, tocol; 3, rtocopherol; 4, $\alpha$-tocopherol; 5, lutein; 6, zeaxanthin; 7, cryptoxanthin; 8, $\alpha$-carotene; 9, all-trans- $\beta$-carotene; 10, cis- $\beta$-carotene 
with a methanol/water/ $n$-butanol mobile phase, based on our previous experience with separation of all-trans and the mono-cis isomers of retinol (26). We found, as have others ( 6 , 7,33 ), that bonded-phase separations are enhanced by the addition of a third solvent possessing polar and nonpolar ends (such as $n$-butanol). The addition of a supporting electrolyte was also beneficial to the separation of these micronutrients. With this gradient separation, all-transretinol is separated at the baseline from its 13-cis isomer, $\alpha$ tocopherol is separated from $\gamma$-tocopherol, and all-trans- $\beta$ carotene is separated, also at the baseline, from the cis isomers and $\alpha$-carotene. Recently, the $15,15^{\prime}$-cis and 9-cis isomers of $\beta$-carotene have been separated from the all-trans and $\alpha$-isomer in determinations in foods (34).

\section{Detection of the Analytes in Serum}

We evaluated the use of LCEC for the determination of the three micronutrients because of the sensitivity and selectivity offered by the technique. LCEC has previously been used for tocopherol determination $(9,19)$. However, a recent tocopherol method (9) noted an unexpected pronounced $\mathrm{pH}$ effect for the hydrodynamic current-voltage curve of the tocopherol isomers, the signal being zero for $\mathrm{pH}$ values $<4$. We examined the $\mathrm{pH}$ dependence of the $\alpha$ tocopherol electrochemical signal by using rotating disk voltammetry over the $\mathrm{pH}$ range of 1.6 to 10 with a glassy carbon disk. A single oxidation wave $\left(E_{1 / 2}=+0.55 \mathrm{~V}\right)$ was found for all $\mathrm{pH}$ values $<8$. Above $\mathrm{pH} 8$, the wave splits, a second wave appearing at lower potential $\left(E_{1 / 2}=+0.35 \mathrm{~V}\right)$; the sum of the wave heights remained constant. At low $\mathrm{pH}$, the oxidation of the protonated form of the phenolic moiety predominated, whereas the second (easier) oxidation wave appeared at the higher $\mathrm{pH}$ because of the oxidation of the phenolate anion $\left(\mathrm{p} K_{a} \approx 10\right)$. The $\mathrm{pH}$ dependence for the electro-oxidation of phenols has been described (35): at $\mathrm{pH}$ values below $\mathrm{p} K_{a}$, the $\mathrm{OH}$ moiety is oxidized to a radical cation in the first oxidation step; at $\mathrm{pH}$ values near or above $\mathrm{p} K_{a}$, the oxidation potential decreases because the deprotonated phenolate anion may be more easily oxidized to a free radical. No change in the protonation level of tocopherol is expected in the $\mathrm{pH}$ range of 2 to 7 , and thus no change in the electro-oxidation signal would be expected. We have concluded that the electro-oxidation of tocopherol shows no $\mathrm{pH}$ dependence from $\mathrm{pH} 2$ to 7 , and thus we use an ammonium acetate buffer ( $\mathrm{pH} 3.5$ ).

Only one preliminary investigation of the use of LCEC for retinol and $\beta$-carotene has been reported (22). Bulk voltammetric studies of the electro-oxidation of $\beta$-carotene $(23,25)$ and retinol $(23,24)$ in nonaqueous solvents have been made. Recently, we investigated the electro-oxidation reaction and the use of LCEC for all-trans-retinol and its cis isomers (26). The electro-oxidation of retinol is a multi-step process, involving the initial formation of radical cations from the double bonds, followed by reaction with the protic solvents, with no involvement of the alcohol moiety. We found little or no selectivity for the electro-oxidation of any one specific double bond, in the five-bond conjugated system. A charge of 4.7 electrons per retinol molecule occurred rapidly in coulometric experiments. A similar sequence of electro-oxidation reactions might be expected for $\beta$-carotene, except the number of electrons transferred may be twice that for retinol (24).

As a basis for LCEC determination of the micronutrients, we constructed a hydrodynamic voltammogram (Figure 3). The current response, measured as peak area, was normal-

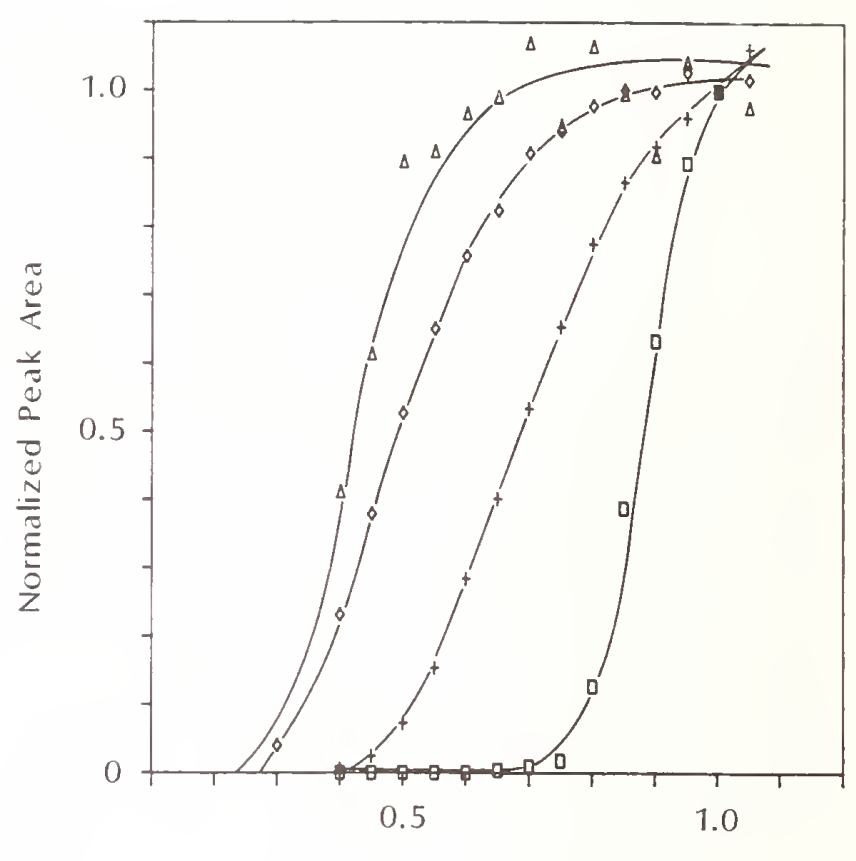

Applied Potential (in V)

Fig. 3. Hydrodynamic voltammograms for all-trans-retinol ( $\square$ ), tocol $(+), \alpha$-tocopherol $(\diamond)$, and all-trans $\beta$-carotene $(\Delta)$ at a glassy carbon electrode

ized by dividing the response at a given potential by the response at $+1000 \mathrm{mV}$. The optimum potential for the simultaneous determination of all three analytes is +900 $\mathrm{mV}$, and is limited by the requirement to detect retinol. For detecting $\alpha$-tocopherol and $\beta$-carotene, an applied potential of only $+0.75 \mathrm{~V}$ would be necessary and would provide additional detector selectivity (see below).

For absorbance detection, we used an instrument that allowed rapid $(<100 \mathrm{~ms})$ wavelength programming to the absorbance maxima for each analyte. The wavelength changes and subsequent auto-zero adjustments were timed to occur at least $3 \mathrm{~min}$ before the elution of each micronutrient (Figure 2), thereby providing the data system an adequate baseline for measurement.

Calibration curves for the three analytes were linear through the physiological concentration range for both the absorbance and electrochemical detectors (Table 1). However, when we used mobile phases containing a large initial proportion of water, the $\beta$-carotene calibration curve displayed some slight curvature in both detectors. Table 2 shows the effect of the initial proportion of water on the $\beta$ carotene signal. The signal was also dependent on the material used in the column inlet frit. We speculate that the mechanism of loss of the $\beta$-carotene signal may involve precipitation of the injected sample onto the oxide surface of the steel frit, with slow redissolution of $\beta$-carotene into mobile phases that contain more water. This speculation is supported by the "tailing" of the $\beta$-carotene peak in solvents in which signal loss occurred.

We compared the detection limits of the absorbance and electrochemical detectors for the three analytes (Table 3), i.e., a signal three times the peak-to-peak baseline noise levels. The detection limits for the electrochemical detector were somewhat superior to absorbance detection for all of the analytes, especially for $\alpha$-tocopherol.

The relative selectivity of the two detectors for the determination of the three analytes in serum may be judged by 
Table 1. Linear Regression Calibration Curves for the Micronutrients by Each Detector

\begin{tabular}{|c|c|c|c|c|c|}
\hline \multirow[b]{2}{*}{ Analyte } & \multirow{2}{*}{$\begin{array}{c}\text { Concn range, } \\
\mathrm{mg} / \mathrm{L}\end{array}$} & \multicolumn{2}{|c|}{ LCUV } & \multicolumn{2}{|c|}{ LCEC } \\
\hline & & SE & Intercept & SE & Intercept \\
\hline $\begin{array}{l}\text { All-trans-retinol } \\
\alpha \text {-Tocopherol } \\
\text { All-trans- } \beta \text {-carotene }\end{array}$ & $\begin{array}{l}0-1.3 \\
0-16 \\
0-1.0\end{array}$ & $\begin{array}{l}0.00841 \\
0.0150 \\
0.0264\end{array}$ & $\begin{array}{l}-0.018 \\
0.0032 \\
-0.10\end{array}$ & $\begin{array}{l}0.0196 \\
0.0109 \\
0.0356\end{array}$ & $\begin{array}{c}-0.011 \\
0.0024 \\
-0.084\end{array}$ \\
\hline$n=14$ each. & & & & & \\
\hline
\end{tabular}

Table 2. Variation of Absorbance Signal for $\beta$-Carotene with Mobile Phase Composition and Frit Type

\begin{tabular}{ccc} 
& \multicolumn{2}{c}{ Absorbance (arb. units) } \\
\cline { 2 - 3 } Initlal proportlon of water & Stainless & \\
In moblle phase, $\mathrm{mL} L$ & 50100 & Teflon frit \\
5 & 45500 & 76000 \\
65 & 39800 & 73400 \\
110 & 34600 & 70100 \\
155 & & 69000 \\
\hline
\end{tabular}

the ability to provide baseline-resolved chromatographic signals (cf. Figures 1 and 2). The selectivity of the absorbance detector was slightly better than the electrochemical detector for all-trans-retinol; however, with the gradientelution solvent program, baseline resolution was possible with both detectors. The electrochemical detector provides superior selectivity for tocol and $\alpha$-tocopherol, as evidenced by small chromatographic shoulders found on the tocol and $\alpha$-tocopherol peaks in some absorbance chromatograms but not observed with the electrochemical detector. We were able to alleviate most of this occasional absorbance detection interference by using cursor-controlled manual integration. The selectivity of the absorbance detector was superior to that of the electrochemical detector for $\beta$-carotene (Figure 4): interfering serum components, including retinol palmitate and possibly other retinol esters, were detected electrochemically (middle trace) but not by absorbance measurements (lowest trace). The interfering retinol esters could be detected by their absorbance at the retinol optimum wavelength, $325 \mathrm{~nm}$ (upper trace). Although the retinol esters interfere in the LCEC at the applied potential of $+900 \mathrm{mV}$, no interference with the $\beta$-carotene signal is observed if a lower detector potential of $+750 \mathrm{mV}$ is used. Perhaps it would be feasible to program a change in potential from +900 to $+750 \mathrm{mV}$ after the elution of retinol and tocol; this would allow the detection of $\alpha$-tocopherol and $\beta$-carotene with maximum signal and better selectivity. Again, we currently rely on cursor-controlled manual integration to

\section{Table 3. Detection Limits for the Analytes}

Concn, $\mu \mathrm{g} / \mathrm{L}$

\begin{tabular}{|c|c|c|}
\hline Analyte & Absorbance & Electrochemlcal \\
\hline All-trans-retinol & $6.0(150)^{a}$ & 4.1 (103) \\
\hline$\alpha$-Tocopherol & $96 \quad(2400)$ & $0.65 \quad(16)$ \\
\hline All-trans- $\beta$-carotene & $(730)$ & (53) \\
\hline
\end{tabular}

- Numbers in parentheses indicate pg of analyte in $25-\mu \mathrm{L}$ injection volume.

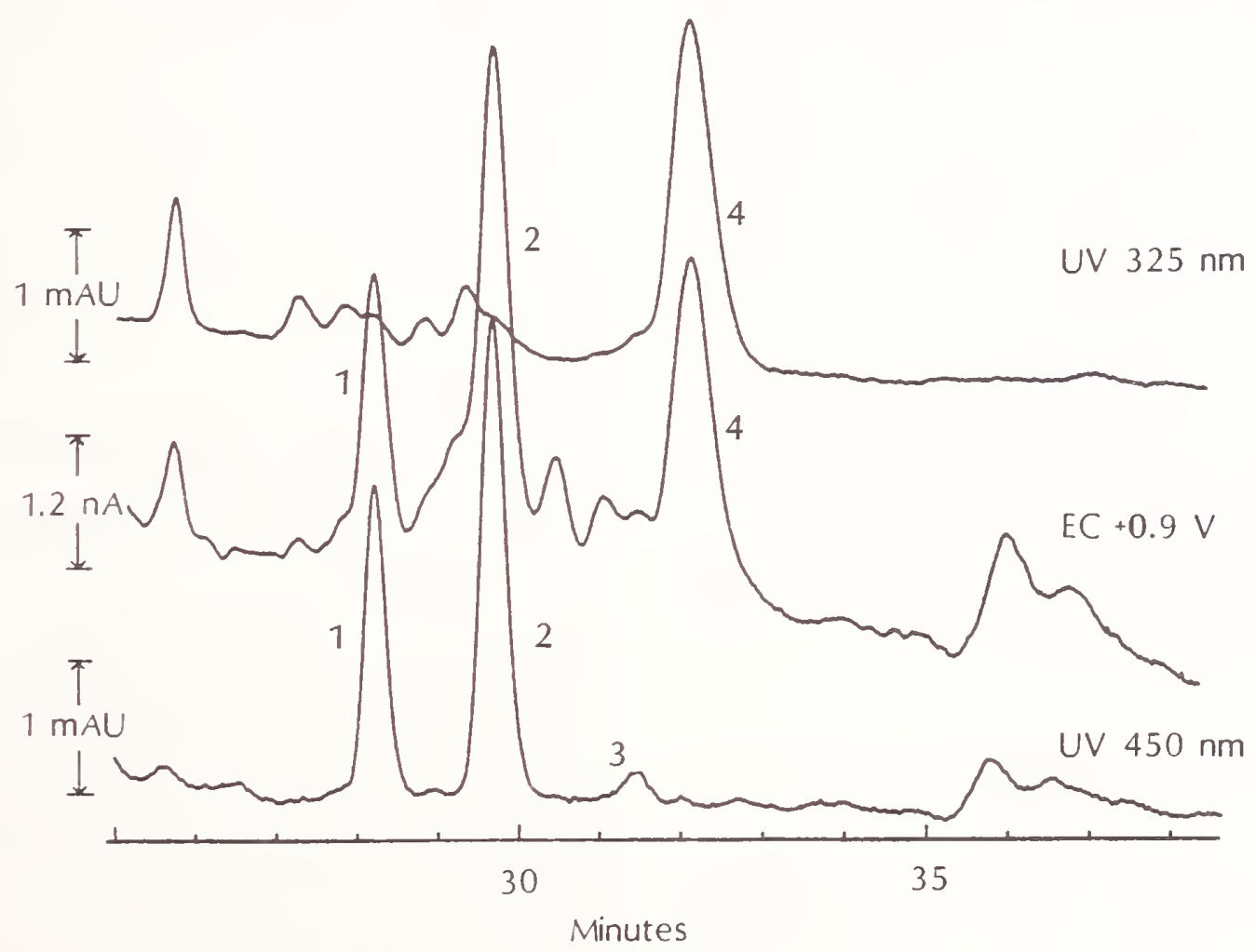

Fig. 4. Comparison of absorbance and electrochemical detection of carotenes

Lowest trace: absortance detection at $450 \mathrm{~nm}$; middle trace: amperometric detection at +900 mV; upper trace: absorbance detection at $325 \mathrm{~nm}$. Peaks: 1 , a-carotene; 2 , all-trans $\beta$-carotene; 3 , cis- $\beta$-carotene; 4 , retinol palmitale 

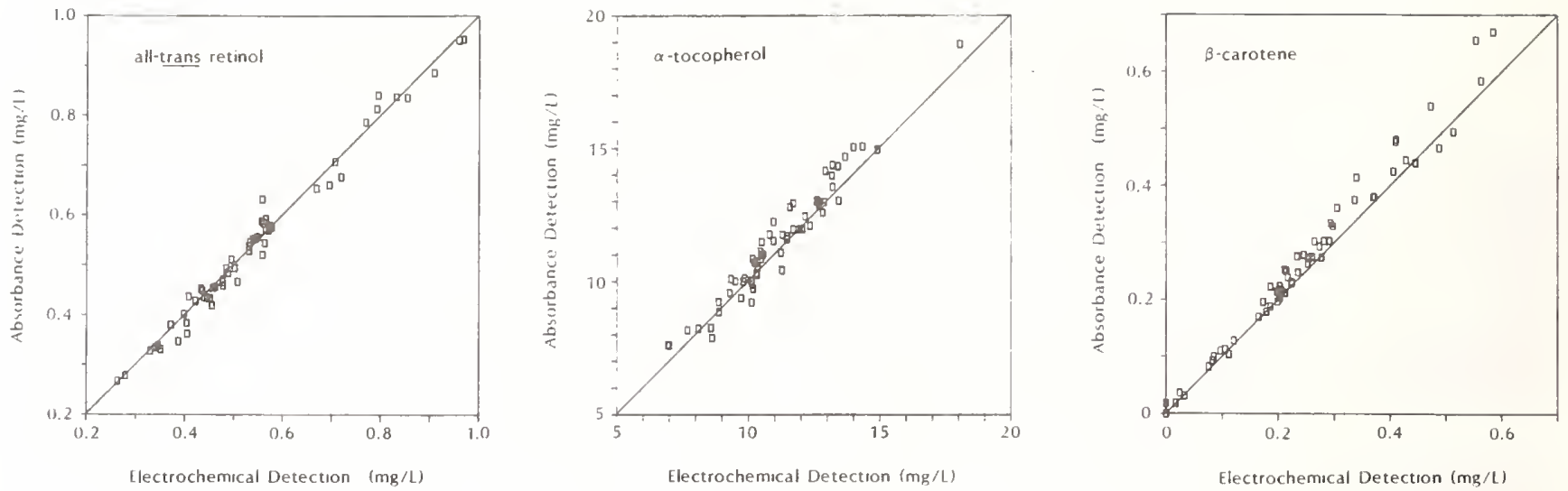

Fig. 5. Companison of the absorbance with the electrochemical detection results for the determination of (leff) all-trans-retinol $(n=72),(m i d d l e) \alpha$-tocopherol $(n=71)$, and (right) $\beta$-carotene $(n=72)$

Regression equation parameters are given in the text

minimize this interference with the $\beta$-carotene signal in the electrochemical detector.

\section{Determination of the Analytes}

We evaluated the method by assaying (all in duplicate) a series of serum samples and one serum pool obtained from 27 Japanese Hiroshima-bomb survivors who have subsequently developed cancer. The procedure was calibrated with a single external standard, at the beginning and at the end of the day, to correct for small amounts of drift in the electrochemical detector response. Correlation plots for the micronutrient values determined with the absorbance and electrochemical detectors are presented in Figure 5. Linear least-squares analysis $(n=72)$ indicates an excellent correlation between the determined values by the two detectors for all-trans-retinol, with a slope of 1.01 and standard error of 0.016 , and for $\alpha$-tocopherol, with a slope of 1.02 and a standard error of 0.025 . However, despite excellent correlation between the detectors, apparently some small bias is indicated for the determination of $\beta$-carotene (slope 1.08, standard error 0.02). Overcompensation in the manual integration of the retinol ester interference with the $\beta$ carotene peak may have produced a negative bias for the electrochemical detector results.

Figure 5 also demonstrates the relative lack of interference in the micronutrient determinations from unknown serum components or ingested drugs. Such interferences are not likely to provide response factors similar to those of the analytes in both detectors and thus would appear in the plots as points far removed from the line of identity.

The results for the analyses of the pooled serum are presented in Table 4. The agreement between the two detectors is good, and the uncertainty (CV) was acceptable $(3 \%$ to $4 \%$ ) for all three analytes.

Table 4. Determination of Micronutrients in a Human Serum Pool

\begin{tabular}{|c|c|c|c|c|c|c|}
\hline & \multicolumn{2}{|c|}{ All-trans-retinol } & \multicolumn{2}{|c|}{ astocopherol } & \multicolumn{2}{|c|}{$\beta$-Carotene } \\
\hline & LCUV & LCEC & LCUV & LCEC & LCuV & LCEC \\
\hline $\bar{X}, \mathrm{mg} / \mathrm{L}$ & 0.563 & 0.554 & 13.5 & 12.9 & 0.216 & 0.198 \\
\hline $\mathrm{CV}, \%$ & 4.1 & 3.0 & 4.7 & 3.2 & 4.1 & 1.4 \\
\hline
\end{tabular}

Although the absorbance and electrochemical detectors measure the three micronutrients by completely separate principles with different attendant interferences, the results for both still depend on the same extraction and separation technique. Absolute validation of the our method would require agreement with the analysis of a serum Standard Reference Material, for which the values of the three micronutrients would have been certified by two totally independent techniques. Current work at NBS is directed toward the development of such a Standard Reference Material.

References

1. Britton G. Greneral carotenoid methods. Methods Enzymol 1985;111:113-49.

2. De Leenheer AP, Lambert WE, De Reuyter MGM. Modern chromatographic analysis of the vitamins. New York: Marcel Dekker, 1984.

3. Matthews-Roth MM, Stampfer MJ. Some factors affecting the determination of carotenoids in serum. Clin Chem 1984;30:459-61.

4. Martinek, RG. Method for the determination of vitamin $\mathrm{E}$ (total tocopherols) in serum. Clin Chem 1964;10:1078-86.

5. Nieremberg DW. Serum and plasma $\beta$-carotene measured with an improved method of high-performance liquid chromatography. $J$ Chromatogr 1985;339:273-84.

6. Driskell WJ, Bashor MM, Neese JW. $\beta$-Carotene determined in serum by liquid chromatography with an internal standard. Clin Chem 1983;29:1042-4.

7. Bieri JG, Brown ED, Smith JCS. Determination of individual carotenoids in human plasma by high performance liquid chromatography. J Liq Chromatogr 1985;8:473-84.

8. Thompson JW, Duval S, Verdier P. Investigation of carotenoids in human blood using high-performance liquid chromatography. $J$ Micronutr Anal 1985;1:81-92.

9. Chou PP, Jaynes KP, Barley LJ. Determination of vitamin $E$ in microsamples of serum by liquid chromatography with electrochemical detection. Clin Chem 1985;31:880-2.

10. Driskell WJ, Lackey AD, Hewett JS, Bashor MM. Stability of vitamin A in frozen sera. Clin Chem 1985;31:871-2.

11. Bankson DD, Russell RM, Sadowski JA. Determination of retinyl esters in serum or plasma by normal-phase liquid chromatography: method and applications. Clin Chem 1986;32:35-40.

12. Driskell WJ, Neese JW, Bryant CC, Bashor MM. Measurement of vitamin $A$ and vitamin $E$ by high-performance liquid chromatography. J Chromatogr 1982;231:439-44.

13. Catignani GL, Bieri JG, Driskell WJ, et al. Proposed Selected Method: simultaneous determination of retinol and $\alpha$-tocopherol in serum or plasma by liquid chromatography. Clin Chem 1983;29:708-12. 
14. Broich CR, Gerber LE, Erdman JW. Determination of lycopene, $\alpha$ - and $\beta$-carotene and retinol esters in human serum by reversephase high-performance liquid chromatography. Lipids $1983 ; 18: 253-8$.

15. Miller $\mathrm{KW}$, Yang CS. An isocratic high-performance liquid chromatography method for the simultaneous analysis of plasma retinol, $\alpha$-tocopherol and various carotenoids. Anal Biochem $1985 ; 145: 21-6$.

16. Miller KW, Lorr NA, Yang CS. Simultaneous determination of plasma retinol, $\alpha$-tocopherol, lycopene, $\alpha$-carotene and $\beta$-carotene by high-performance liquid chromatography. Anal Biochem $1984 ; 138: 340-5$.

17. Nieremberg D, Lester DC. Determination of vitamins A and E in serum and plasma using a simplified clarification procedure and high-performance liquid chromatography. J Chromatogr $1985 ; 345: 275-84$.

18. Milne DB, Botnen J. Retinol, $\alpha$-tocopherol, lycopene and $\alpha$ - and $\beta$-carotene simultaneously determined in plasma by isocratic liquid chromatography. Clin Chem 1986;32:874-6.

19. Ueda T, Igarashi O. Evaluation of the electrochemical detector for the determination of tocopherols in feeds by high-performance liquid chromatography. J Micronutr Anal 1985;1:31-8.

20. Russell MJ, Thomas BS, Wellock E. Simultaneous assay of serum vitamin $\mathrm{A}$ and vitamin $\mathrm{E}$ by high performance liquid chromatography using time-switched UV and fluorimetric detectors. J High Resolut Chromatog Commun Chromatog Commun 1986;9:281-4.

21. Williams ATR. Simultaneous determination of serum vitamin $A$ and $E$ by liquid chromatography with fluorescence detection. $J$ Chromatogr 1985;341:198-201.

22. Ikenoya S, Abe K, Tsuda T, et al. Electrochemical detector for high performance liquid chromatography. II. Determination of tocopherols, ubiquinones and phylloquinone in blood. Chem Pharm Bull 1979;27:1237-44.

23. Park SM. Electrochemical studies of $\beta$-carotene all-trans retinal, and all-trans retinol in tetrahydrofuran. J Electrochem Soc $1978 ; 125: 216-22$.

24. Mairanovskii VG, Vakulova CA, Samokhvalov GI. Polarogra. phy of conjugated systems in dimethylformamide IV. Mechanism of the reduction of retinol and related substances: polarography and electrophoresis at a controlled potential. Elektrochimiya 1967:2331.

25. Mairanovskii VG, Engovatov AA, Ioffe NT, Samokhvalov GI. Electron-donor and electron-acceptor properties of carotenoids. J Electroanal Chem 1975;66:123 -37 .

26. MacCrehan WA, Schönberger E. Reverse-phase HPLC separation and electrochemical detection of retinol and its isomers. J Chromatogr 1987;417:65-78.

27. Brunius E. Assay of vitamin oils. A report on three collaborative experiments. J Assoc Off Anal Chem 1959;42:657-78.

28. Isler $O$, Lindlar $H$, Montavon $M$, et al. Synthesis in der Carotinoidreine. Die technische Syntheses von Carotin. Helv Chim Acta 1956;39:249-59.

29. De Leenheer AP, De Bevere V, De Reuyter MGM, Claeys AE. Simultaneous determination of retinol and $\alpha$-tocopherol in human serum by high-performance liquid chromatography. J Chromatogr 1979;162:408-13.

30. Khachik F, Beecher GR. Synthesis of C-45- $\beta$-carotene, a potentially useful internal standard for quantitation of hydrocarbon carotenoids by HPLC. J Ind Eng Chem 1986;25:671-5.

31. Khachik F, Beecher GR, Whittaker NF. Separation, identification and quantification of the major carotenoid and chlorophyll constituents in extracts of several green vegetables by liquid chromatography. J Agric Food Chem 1986;603-16.

32. Yang CS. Advantages and disadvantages of single and multivitamin analytical methods. Second NBS/NCI micronutrient workshop. Gaithersburg, MD, May 28-9, 1986.

33. Bhat PV, Co HT, Lacroix A. Effect of 2-alkanols on the separation of geometric isomers of retinol in non-aqueous highperformance liquid chromatography. J Chromatogr 1982;260:129 36.

34. Bushway RJ. Determination of $\alpha$ - and $\beta$-carotene in some raw fruits and vegetables by high-performance liquid chromatography. J Agric Food Chem 1986;34:409-12.

35. Adams RN. Electrochemistry at solid electrodes. New York: Marcel Dekker, 1969. 


\title{
Separation and Relative Distribution of All-trans- $\beta$-Carotene and its cis Isomers in $\beta$-Carotene Preparations
}

\author{
Neal E. Craft, ${ }^{a *}$ Lane C. Sander ${ }^{a} \&$ Herbert F. Pierson ${ }^{a, b}$ \\ ${ }^{a}$ Center for Analytical Chemistry, National Institute of Standards and Technology, \\ Gaithersburg, Maryland 20899, USA \\ ${ }^{b}$ Diet and Cancer Branch, National Cancer Institute, Rockville, Maryland 20852, USA
}

(Received 6 September 1989; revised version received 29 August 1990; accepted 25 September 1990)

\begin{abstract}
The purity and relative distribution of $\beta$-carotene isomers in several commercially available preparations of $\beta$-carotene was evaluated using spectrophotometric methods and reversed-phase liquid chromatography (LC). The purity of five lots of $\beta$-carotene evaluated by comparison of spectrophotometric to gravimetric determinations was found to range from $7 \cdot 1$ to $82.9 \%$. Reversed-phase LC was then used to measure the impurities due to other carotenoids and geometric isomers of $\beta$-carotene which also absorb in the $450 \mathrm{~nm}$ region. Five different reversed-phase $C_{18}$ columns were examined for their ability to resolve the geometric isomers of $\beta$-carotene which contribute to the apparent concentration of trans- $\beta$-carotene standard solutions. The columns packed with wide-pore, $C_{18}$ polymerically-modified silica exhibited adequate selectivity to separate the geometric isomers from all-trans- $\beta$ carotene. Impurities separated by LC accounted for 16-75\% of the absorbance of commercial $\beta$-carotene preparations at $450 \mathrm{~nm}$. Based on these observations, all-trans- $\beta$-carotene measurements could potentially be only $1 / 50$ of reported values.
\end{abstract}

Key words: $\beta$-carotene, carotenoids, HPLC, isomers, purity.

*To whom correspondence should be addressed.

209

Journal of Micronutrient Analysis 0266-349X/91/\$03.50 (C) 1991 Elsevier Science Publishers Ltd, England. Printed in Northern Ireland 


\section{INTRODUCTION}

Although a multitude of methods have been reported for carotenoid measurements, only a few address the separation of geometric isomers. The most complete separations of carotenoid isomers have been accomplished by liquid chromatography (LC) using lime $\left(\mathrm{Ca}(\mathrm{OH})_{2}\right)$ and alumina columns (Vecchi et al., 1981; Tsukida et al., 1981; Tsukida \& Saiki, 1982; Chandler \& Schwartz, 1987; 1988). Tsukida and co-workers resolved $17 \beta$-carotene isomers using a column packed in their laboratory with a hand-selected batch of lime (Tsukida \& Saiki, 1982; Tsukida et al., 1981). Unfortunately, commercially prepared $\mathrm{Ca}(\mathrm{OH})_{2}$ columns are not available and extensive batch-to-batch variability of $\mathrm{Ca}(\mathrm{OH})_{2}$ has been reported (Chandler \& Schwartz, 1987). Vecchi et al. (1981) separated 12 isomers of $\beta$-carotene using an alumina column; however, significant modifications of normal LC equipment were required to regulate the mobile phase temperature and water content. Successful quantitative analysis requires that the LC column selectivity must be sufficient to separate the standard of interest from all interferences contributing absorbance at the detection wavelength, including geometric isomers. Our goal was to identify a means of assessing carotene purity for laboratories making routine measurements. Reversed-phase LC is the method most commonly used to measure carotenoids. This technique offers the capability to separate a wide range of compounds and $\mathrm{C}_{18}$ stationary phases are compatible with most solvents. The resolution of carotenoid isomers using reversed-phase columns is less complete than that obtained using lime and alumina columns; however, the columns are commercially available and the equipment requires no special modifications.

The investigation of $c$ is isomers of $\beta$-ca rotene was further motivated by the lack of knowledge about the carotenoid isomer content of natural and commercial $\beta$-carotene products. Since much of the association between cancer prevention and $\beta$-carotene is based on epidemiological and food consumption data, it is essential to determine more accurately which isomers are present naturally in foods and the composition of commercially available $\beta$-carotene products. The development of methods that resolve carotenoid isomers is imperative to understanding which carotenoid(s)/isomer(s) might be beneficial for human cancer prevention. Therefore, the ability of several reversed-phase $\mathrm{C}_{18}$ columns to separate $\beta$-carotene isomers was investigated. Commercial preparations of $\beta$-carotene were then analyzed to determine their relative content of $\beta$-carotene isomers using spectrophotometry and reversed-phase LC with absorbance detection. 
TABLE 1

Source and Description of $\beta$-Carotene Products Investigated ${ }^{a}$

\begin{tabular}{|c|c|c|}
\hline Supplier & Lot & Description \\
\hline Fluka $^{b}$ & 245663582 & Synthetic crystalline \\
\hline Sigma $^{b}$ & $83 \mathrm{~F} 0218$ & Type III crystalline from carrots \\
\hline Sigma & $15 F 9715$ & Type IV crystalline from carrots \\
\hline Sigma $^{b}$ & $112 \mathrm{~F} 0307$ & Type I synthetic crystalline \\
\hline MCB & 2259 & Synthetic crystalline \\
\hline ICN & 14008 & Synthetic crystalline \\
\hline Kodak & E15A & Synthetic crystalline \\
\hline * & & Betatene $4 \%$ algal $\beta$-carotene suspension \\
\hline * & & Betatene $30 \%$ algal $\beta$-carotene suspension \\
\hline * & 1 & Betatene 10000 IU algal $\beta$-carotene capsule \\
\hline * & 2 & Betatene $10000 \mathrm{IU}$ algal $\beta$-carotene capsule \\
\hline * & & Chinese crystalline \\
\hline * & & Vita crystalline \\
\hline * & & Roche $10 \%$ beadlets \\
\hline
\end{tabular}

${ }^{a}$ A detailed investigation of lot-to-lot reproducibility has not been performed. Therefore the lots analyzed may or may not be representative of the manufacturer's products.

${ }^{b} \beta$-Carotene preparations not measured by LC.

* Samples provided by the National Cancer Institute, Division of Cancer

Prevention and Control.

\section{MATERIALS AND METHODS}

The descriptions, lot numbers, and suppliers of the $\beta$-carotene products analyzed are listed in Table 1. All solvents used for the LC analyses were HPLC grade or equivalent and were used without further treatment.

\section{Gravimetric determinations}

Weighed samples (c. $0.35 \mathrm{mg}$ ) from five lots of $\beta$-carotene were each dissolved in $500 \mu 1$ of toluene by ultrasonic agitation and diluted to $50 \mathrm{ml}$ with acetonitrile. The UV/visible spectrum for each sample was recorded immediately using a spectrophotometer (Hewlett-Packard model 8450A). Spectrophotometric concentrations were calculated using published absorptivities for $\beta$-carotene in ethanol (Isler et al., 1956) since absorptivities have not been reported in acetonitrile. Using a Cary Model 219 spectrophotometer (Varian Instruments, Walnut Creek, CA), we found that the absorptivity of $\beta$-carotene in the two solvents varied by less than $1 \%$ and the wavelength maxima in acetonitrile was $1-2 \mathrm{~nm}$ higher than in ethanol. An absorptivity in acetonitrile is not reported here due to 
the presence of impurities in all $\beta$-carotene samples examined. The relative purity of preparations was determined by comparing the spectrophotometric concentration to the gravimetric concentration.

\section{LC sample preparation}

$\beta$-Carotene materials were received as either crystalline material or as an oily suspension. A representative sample of each product was dissolved in absolute ethanol. Ethanol was the solvent of choice since it is both miscible with the LC mobile phase, has not been reported to induce carotene isomerization, and ethanolic $\beta$-carotene solutions have demonstrated stability in our laboratory. The Hoffman-La Roche $\beta$-carotene beadlets are gelatin-coated which necessitated the removal of the gelatin prior to extraction. This was accomplished either by heating the beadlets in ethanol to $50^{\circ} \mathrm{C}$ to dissolve the gelatin, permitting the extraction of $\beta$-carotene into the ethanol, or denaturing the gelatin with $6 \mathrm{M}$ urea followed by hexane extraction. The hexane extract was evaporated using a stream of $\mathrm{N}_{2}$ and redissolved in ethanol.

\section{Equipment*}

The liquid chromatographic system consisted of a ternary single-piston pumping system fitted with a fixed-loop injector. Columns investigated

TABLE 2

LC Column Characteristics ${ }^{a}$

\begin{tabular}{lccccc}
\hline Column & $\begin{array}{c}\text { Particle } \\
\text { size }(\mu \mathrm{m}) \text { \& type }\end{array}$ & $\begin{array}{c}\text { Carbon } \\
\text { load }(\%)\end{array}$ & $\begin{array}{c}\text { Synthesis } \\
\text { type }\end{array}$ & $\begin{array}{c}\text { End- } \\
\text { capped }\end{array}$ & $\begin{array}{c}\text { Pore } \\
\text { size }(\mathcal{A})\end{array}$ \\
\hline Vydac 201TP C 18 & 5 , spherical & 8 & Polymeric & - & 300 \\
Bakerbond & 5, spherical & 10 & Polymeric & + & 300 \\
$\quad$ Widepore $C_{18}$ & 10, irregular & 10 & Monomeric & + & $50-300$ \\
$\mu$ Bondapak $C_{18}$ & 5, spherical & 13 & Monomeric & + & 80 \\
Ultrasphere $C_{18}$ & 5, spherical & 20 & Monomeric & - & 70 \\
Zorbax $\mathrm{C}_{18}$ & & & & & \\
\hline
\end{tabular}

${ }^{a}$ Nominal data provided by the column manufacturer.

${ }^{b}$ Monomeric synthesis binds monochlorosilanes to the base silica gel yielding a monolayer.

Polymeric synthesis binds trichlorosilanes to the base silica gel yielding a polymer layer.

* Certain commercial equipment, instruments, or materials are identified in this paper to specify adequately the experimental procedure. Such identification does not imply recommendation or endorsement by NIST, nor does it imply that the materials or equipment identified are necessarily the best available for the purpose. 
for the separation of $\beta$-carotene isomers included Bakerbond Wide Pore $\mathrm{C}_{18}(250 \times 4.6 \mathrm{~mm}$, J. T. Baker Chemical Co., Phillipsburg, NJ), $\mu$ Bondapak $\mathrm{C}_{18}(300 \times 3.9 \mathrm{~mm}$, Waters Division of Millipore, Milford, MA), Ultrasphere C-18 $(250 \times 4.6 \mathrm{~mm}$, Beckman Instruments, San Ramon, CA), Vydac $201 \mathrm{TP}(250 \times 4.6 \mathrm{~mm}$, The Separations Group, Hesperia, CA), and Zorbax ODS $(250 \times 4.6 \mathrm{~mm}$, MacMod Analytical, Inc., Chadds Ford, PA). Additional physical characteristics of these $C_{18}$ stationary phases are listed in Table 2 . Column selectivity for carotenoid isomer separation was tested using methanol or acetonitrile as the primary mobile phase solvent. Ethyl acetate or water was added as a mobile phase modifier to maintain the capacity factor $\left(k^{\prime}\right)$ for $\beta$-carotene in the range of 7.5-12.5. The Vydac 201TP column was ultimately used for the analysis of the commercial $\beta$-carotene preparations with a mobile phase comprised of methanol/water $(97: 3, \mathrm{v} / \mathrm{v})$ at a flow rate of $1.5 \mathrm{ml} \mathrm{min}{ }^{-1}$. Carotenoids were monitored at $450 \mathrm{~nm}$ (absorbance maximum of trans $\beta$-carotene in the mobile phase for the LC detectors) using both a programmable variable wavelength absorbance detector (Spectroflow 783, Kratos/ABI, Ramsey, NJ) and a UV/visible photodiode array detector (H-P 1040A, Hewlett-Packard, Palo Alto, CA) in series. Data were collected by computer on floppy discs permitting the reprocessing of chromatograms. The relative standard deviation of the trans- $\beta$-carotene peak area in a $5 \mu \mathrm{g} \mathrm{ml}^{-1}$ standard solution was $1.5 \%$ for 10 injections of $20 \mu 1$.

\section{Peak identification}

$\beta$-Carotene isomers were tentatively identified by UV/visible absorbance spectra, Q-ratios and elution order from a $\mathrm{Ca}(\mathrm{OH})_{2}$ column. Q-ratios, absorbance at the wavelength maximum/absorbance at the 'cis' peak, were calculated from spectral data obtained using the photodiode array detector (PAD) and compared to published values (Tsukida \& Saiki, 1982; Quackenbush, 1987). Elution order of the major isomers (9-cis and 13-cis) from $\mathrm{Ca}(\mathrm{OH})_{2}$ was compared to published separations (Tsukida et al., 1981; Chandler \& Schwartz, 1987). A column $(4.6 \times 250 \mathrm{~mm})$ packed with $\mathrm{Ca}(\mathrm{OH})_{2}$ (J. T. Baker, Phillipsburg, NJ) at 12000 psi was eluted with $1 \%$ acetone in pentane. Peaks corresponding to 9-cis and 13cis isomers of thermally isomerized $\beta$-carotene were collected and chromatographed using the reversed-phase system described above.

\section{RESULTS AND DISCUSSION}

The purity of five lots of $\beta$-carotene was evaluated by comparing the concentration calculated using the absorptivity to the gravimetric 
TABLE 3

Spectrophotometric Concentration of $\beta$-Carotene Products Relative to the Gravimetric Concentrations

\begin{tabular}{cc}
\hline Supplier & Percentage $^{a}$ \\
\hline 1 & $7 \cdot 1$ \\
$2 \mathrm{a}$ & 28.4 \\
$2 \mathrm{c}$ & $43 \cdot 1$ \\
3 & $72 \cdot 3$ \\
4 & $82 \cdot 9$
\end{tabular}

a Spectrophotometric concentration/ gravimetric concentration $\times 100$.

concentration. Estimated purities were low (Table 3), ranging from $7 \cdot 1$ to $82.9 \%$, in spite of an over-estimation of purity due to the absorbance contributed by contaminating carotenoids and $\beta$-carotene isomers. Quackenbush and Smallidge (1986) also observed a similar degree of variability in $\beta$-carotene preparations from six different suppliers.

Five commercially available $C_{18}$ columns were investigated for the separation of a mixture of $\beta$-carotene isomers. The physical characteristics of the different stationary phases are summarized in Table 2. Wide-pore polymeric columns were less retentive than monomeric columns. The mobile phase compositions used to yield a $k^{\prime}$ of approximately 9 for trans- $\beta$-carotene were as follows: Vydac 201TP and Bakerbond WP, methanol/water $(98: 2, \mathrm{v} / \mathrm{v}) ; \mu$ Bondapak 100 methanol; Ultrasphere, methanol/ethyl acetate $(90: 10, \mathrm{v} / \mathrm{v}) ;$ Zorbax, methanol/ethyl acetate $(85: 15, \mathrm{v} / \mathrm{v})$. None of the monomeric columns used in this study possessed adequate selectivity to resolve $\operatorname{cis}-\beta$-carotene isomers from trans- $\beta$-carotene. These observations are in agreement with the reports of others (Quackenbush \& Smallidge, 1986; Quackenbush, 1987; Bushway, $1985)$ and are illustrated in Fig. 1. The mixture of $\beta$-carotene isomers was separated by the wide-pore polymeric $\mathrm{C}_{18}$ columns but not by the monomeric $\mathrm{C}_{18}$ columns. Based on the dimensions of the rigid $\beta$ carotene molecule ( $33 \AA$ long), the narrow pore size of these monomeric columns may limit the accessibility of the molecule to the bonded phase inside the pores. MacCrehan \& Schönberger (1987), Ben-Amotz et al. (1988), Quackenbush (1987) and Bushway (1985) have all reported the separation of trans- $\beta$-carotene from its $c$ is isomers by reversed-phase LC. In all cases the LC columns used to achieve these separations contained wide-pore $\mathrm{C}_{18}$ modified silica synthesized with trichlorosilanes (polymeric synthesis) rather than silica modified with monochlorosilanes (monomeric synthesis). The unique selectivity of polymeric $\mathrm{C}_{18}$ columns 


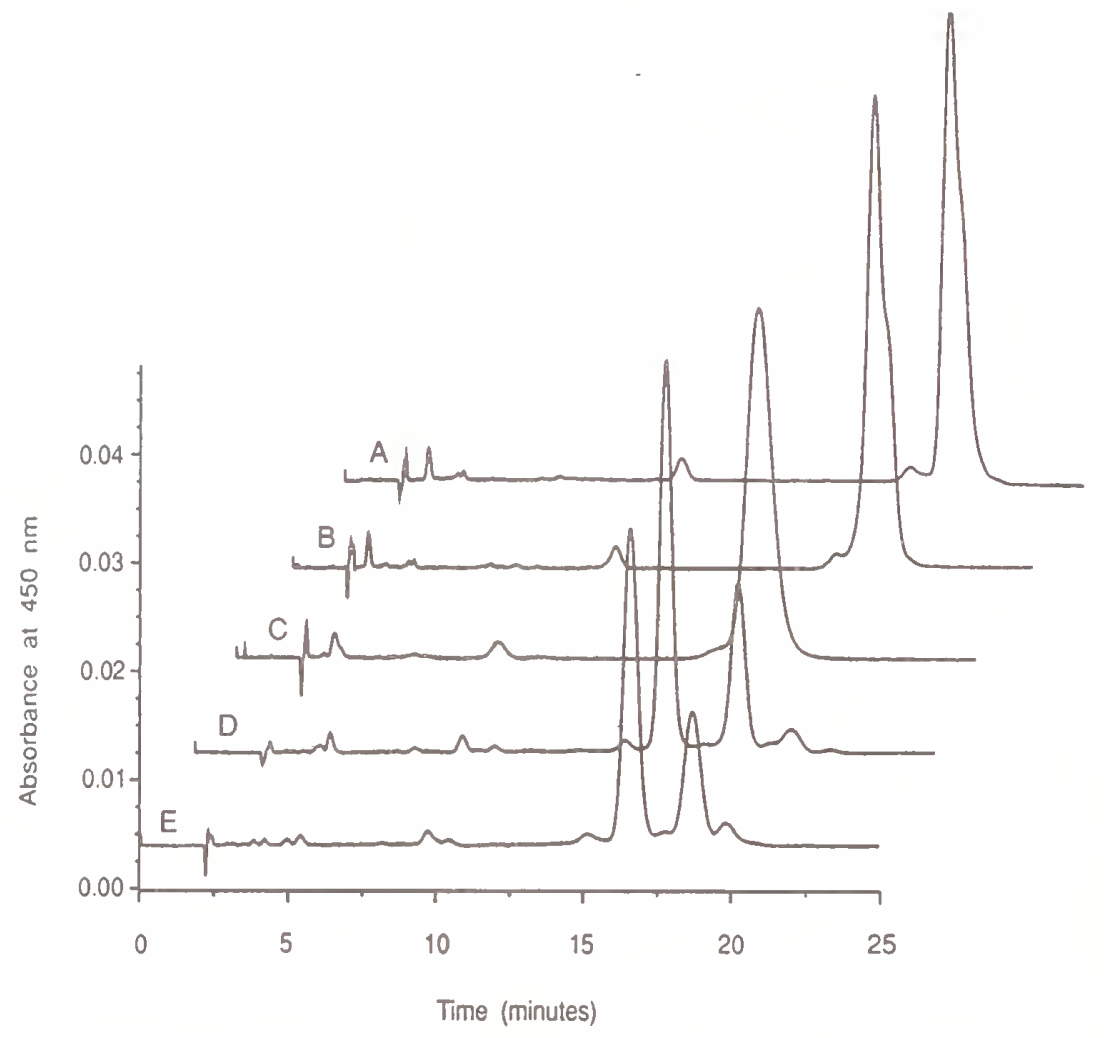

Fig. 1. Comparison of the separation of an algal $\beta$-carotene extract using reversed-phase $\mathrm{C}_{18} \mathrm{LC}$ columns. For all columns, the flow rate was $1.5 \mathrm{ml} \mathrm{min} \mathrm{m}^{-1}$ and eluents were monitored at $450 \mathrm{~nm}$; A Zorbax ODS, methanol/ethyl acetate $(85: 15, \mathrm{v} / \mathrm{v})$; B Ultrasphere ODS, methanol/ethyl acetate (90:10,v/v); C $\mu$ Bondapak, 100 methanol; D Bakerbond WP ODS, methanol/water (98:2, v/v); E Vydac 201TP, methanol/water (98:2, v/v).

for the separation of structurally similar compounds has been investigated extensively for polycyclic aromatic hydrocarbons (PAHs) (Wise et al., 1983; Sander \& Wise, 1984, 1986; Wise \& Sander, 1985). Wise \& Sander (1985) have demonstrated the ability of polymeric $\mathrm{C}_{18}$ columns to resolve isomers of PAH that could not be separated by monomeric columns. These authors have also investigated the influence of pore size, surface coverage and chain length on the PAH isomer separation and found the polymeric synthesis to be the most significant determinant. The effects of specific stationary phase modifications (i.e. pore size, surface coverage, chain length) on the separation of $\beta$-carotene isomers will be described in a later publication.

Mobile phase composition was manipulated to maximize the selectivity for $\beta$-carotene isomers. Although acetonitrile has lower viscosity than methanol resulting in higher efficiency, methanol yielded better selectivity and better recovery of $\beta$-carotene isomers. The average recovery of $\beta$-carotene in acetonitrile-based solvents was $74 \%$ while recovery in methanol-based solvents was $89 \%$. The separation factors, $\alpha$, for $\beta$-carotene $/ \alpha$-carotene and isomer $2 / \beta$-carotene are larger in the 


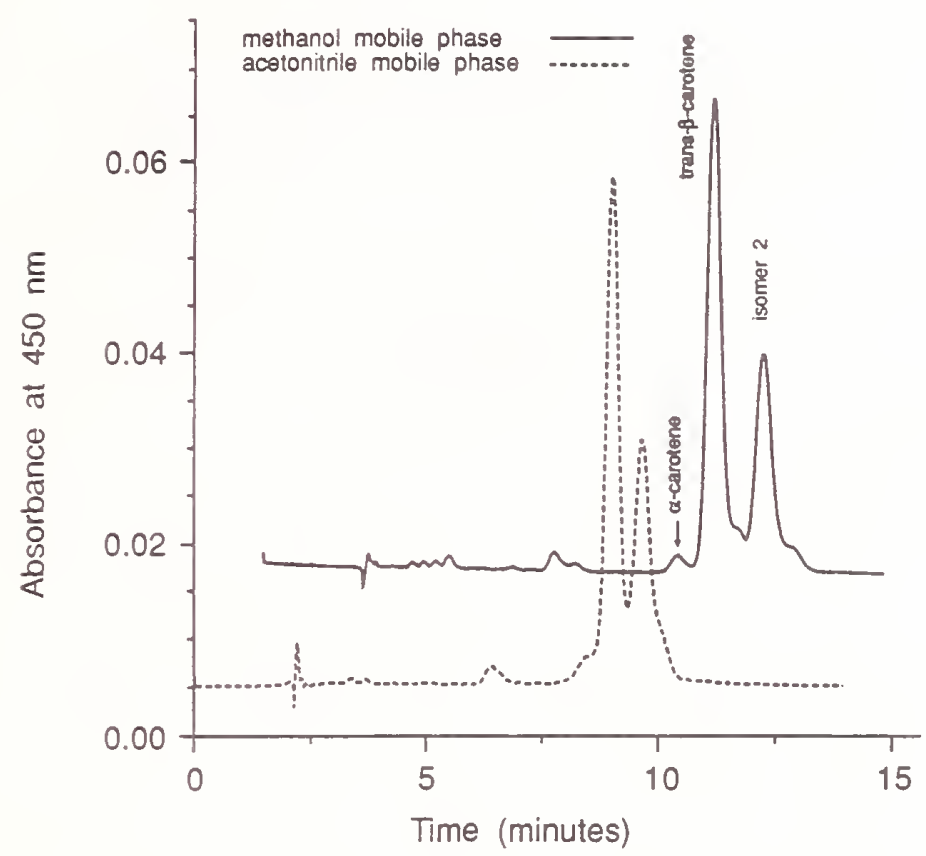

Fig. 2. Comparison of the difference in the separation selectivity using methanol (solid line) or acetonitrile (dashed line) as the mobile phase. Algal $\beta$-caroten z extract injected on a wide-pore polymerically modified reversed-phase $\mathrm{C}_{18} \mathrm{LC}$ column and monitored at $450 \mathrm{~nm}$.

methanol mobile phase than the acetonitrile mobile phase. The difference in selectivity using the two solvents is illustrated in Fig. 2. Therefore, the primary mobile phase constituent was restricted to methanol. Optimal selectivity and recovery are essential for accurate standardization and quantification since the isomers of $\beta$-carotene have different extinction coefficients and absorption maxima which can influence the value assigned to the ' $\beta$-carotene peak'.

Representative chromatograms from the reversed-phase separation of four different $\beta$-carotene products are presented in Fig. 3. Both the relative purity and isomer content of the $\operatorname{commercial} \beta$-carotene reagents vary significantly among the various sources. The composition of the products evaluated in this work by reversed-phase LC, expressed as the area per cent of the totalled peak area absorbance at $450 \mathrm{~nm}$, is listed in Table 4. Trans- $\beta$-carotene constituted from 22 to $84 \%$ and the cis component comprised at least $10 \%$ in all products tested. Quackenbush $\&$ Smallidge (1986) also reported significant variability in the purity and isomer content of commercial $\beta$-carotene compounds. The presence of isomers in $\beta$-carotene products coincides with a recent report by Mayne \& Parker (1988) who observed the presence of cis isomers in commercial preparations of canthaxanthin. However, it refutes their comment that isomers are not associated with $\beta$-carotene preparations. 


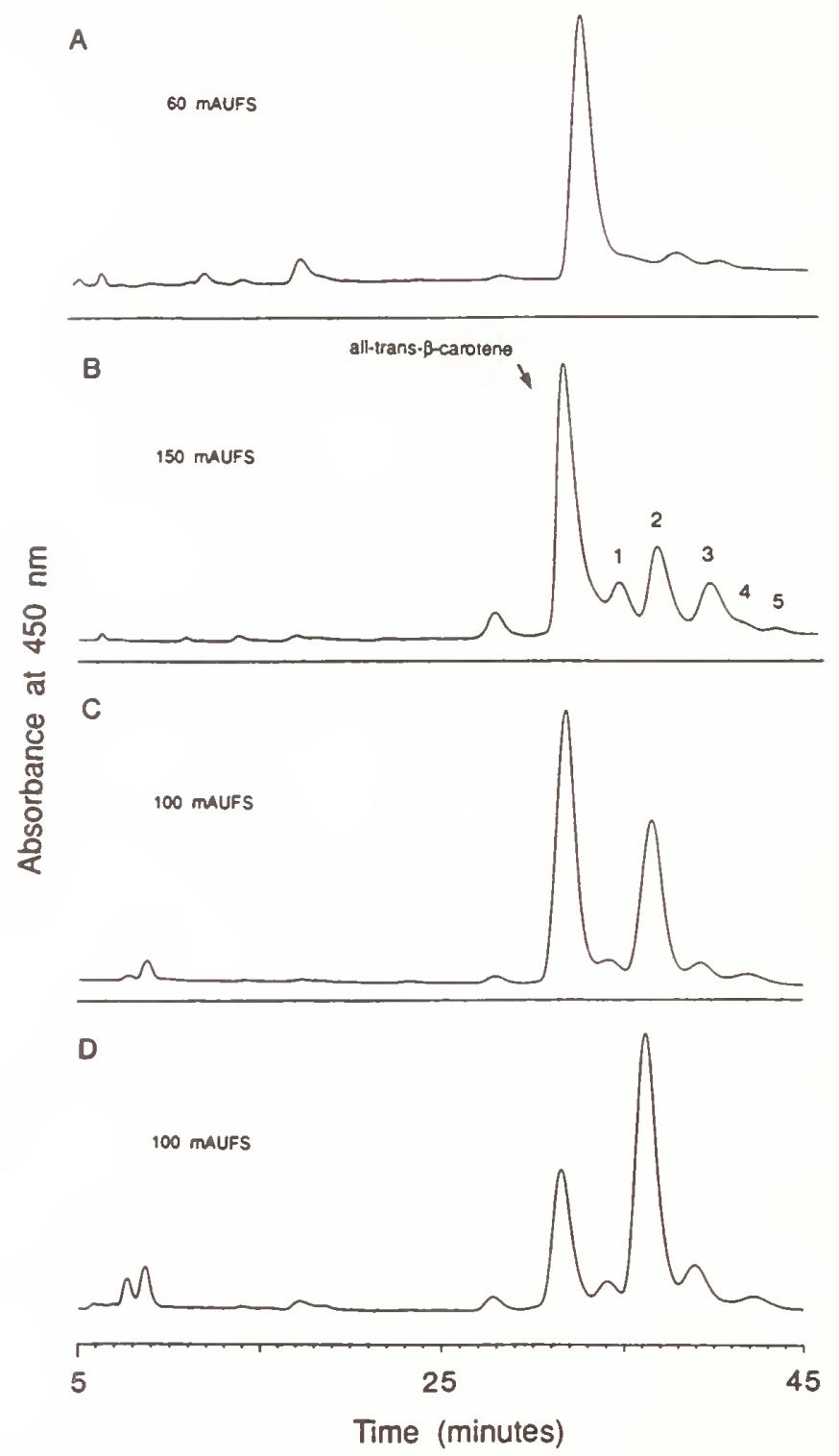

Fig. 3. Representative chromatograms of $\beta$-carotene preparations separated using a wide-pore, polymeric $C_{18}$ column. Mobile phase methanol/water $(97: 3, v / v)$ at a flow rate $1.5 \mathrm{ml} \mathrm{min} \mathrm{m}^{-1}$ and monitored at $450 \mathrm{~nm}$. Cis isomers of $\beta$-carotene are numbered in the order of elution from the LC column. $A=$ Product $2 b, B=$ Product $9 H, C=$ Product $6 b$, $\mathrm{D}=$ Product $6 \mathrm{a}$.

The isomer content of product 9 differed significantly with the type of extraction used (Table 4, $9 \mathrm{C}$ vs. $9 \mathrm{H}$ ). The large cis component observed in the 'hot extract' $(9 \mathrm{H})$ is possibly the result of induced isomerization due to the elevated extraction temperature. Heating is one method that has been used to induce isomerization of carotenoids; however, the temperatures used here are much lower than those typically used to cause isomerization. When an ethanolic solution of crystalline $\beta$-carotene 
TABLE 4

LC Determination of the Relative Composition of $\beta$-Carotene Isomers in $\beta$-Carotene Preparations

\begin{tabular}{|c|c|c|c|c|c|c|c|c|}
\hline \multirow[t]{3}{*}{ Product } & \multicolumn{8}{|c|}{$\%$ Composition $^{a}$} \\
\hline & \multirow[t]{2}{*}{ trans } & \multicolumn{5}{|c|}{ cis isomers } & \multirow{2}{*}{$\begin{array}{c}\text { Total } \\
\text { cis }\end{array}$} & \multirow[t]{2}{*}{ Othert } \\
\hline & & 1 & 2 & 3 & 4 & 5 & & \\
\hline 8 & 84 & 2 & 2 & 5 & - & 1 & 10 & 6 \\
\hline $9 \mathrm{C}$ & 77 & - & 6 & 7 & - & - & 13 & 10 \\
\hline $2 b$ & 72 & - & 8 & 4 & - & - & 12 & 16 \\
\hline 3 & 68 & 3 & 5 & 1 & 2 & - & 12 & 29 \\
\hline 7 & 64 & 2 & 14 & 6 & 2 & - & 24 & 12 \\
\hline 4 & 61 & 5 & 5 & 2 & 2 & - & 14 & 25 \\
\hline 5 & 61 & - & 13 & 8 & - & - & 20 & 19 \\
\hline $6 b$ & 49 & 9 & 30 & 6 & 1 & - & 46 & 5 \\
\hline $9 \mathrm{H}$ & 48 & - & 18 & 13 & - & 1 & 41 & 11 \\
\hline $6 c$ & 33 & 5 & 45 & 4 & 3 & - & 57 & 10 \\
\hline $6 \mathrm{~d}$ & 25 & - & 56 & 8 & 1 & - & 65 & 10 \\
\hline $6 a$ & 25 & 5 & 49 & 10 & 3 & - & 67 & 8 \\
\hline
\end{tabular}

${ }^{a}$ Based on the $\%$ area under the chromatographic peak relative to the total area of all peaks at $450 \mathrm{~nm}$. Values rounded to the nearest whole number.

${ }^{b}$ The 'other' category constitutes primarily $\alpha$-carotene and other $450 \mathrm{~nm}$ absorbing compounds that elute prior to all-trans- $\beta$-carotene.

was submitted to similar conditions, only a $5 \%$ change was observed in the trans isomer.

Of particular note was the very high cis isomer content in some algal $\beta$-carotene products $(6 \mathrm{a}-\mathrm{d})$. The proportion of isomer 2 in products $6 \mathrm{a}, 6 \mathrm{c}$ and $6 \mathrm{~d}$ is greater than the proportion of trans- $\beta$-carotene. The content of isomer 2 is higher than any isomers reportedly formed by deliberate isomerization of trans- $\beta$-carotene, negating the possibility of it being an artifact (Zechmeister, 1962). The high ratio of cis/trans along with other factors influencing the isomer distribution of algal $\beta$-ca rotene have been reported previously by Ben-Amotz et al. (1988). Using the UV/visible absorbance spectra collected by the photodiode array detector during the LC separation, it was possible to compare the Q-ratio (absorbance at the wavelength maximum/absorbance at the 'cis' peak) to published values (Tsukida \& Saiki, 1982; Quackenbush, 1987). The primary cis isomer (peak 2 ) has been tentatively identified as 9 -cis- $\beta$-carotene by its Q-ratio, absorption spectrum, and elution from $\mathrm{Ca}(\mathrm{OH})_{2}$. This identifi- 


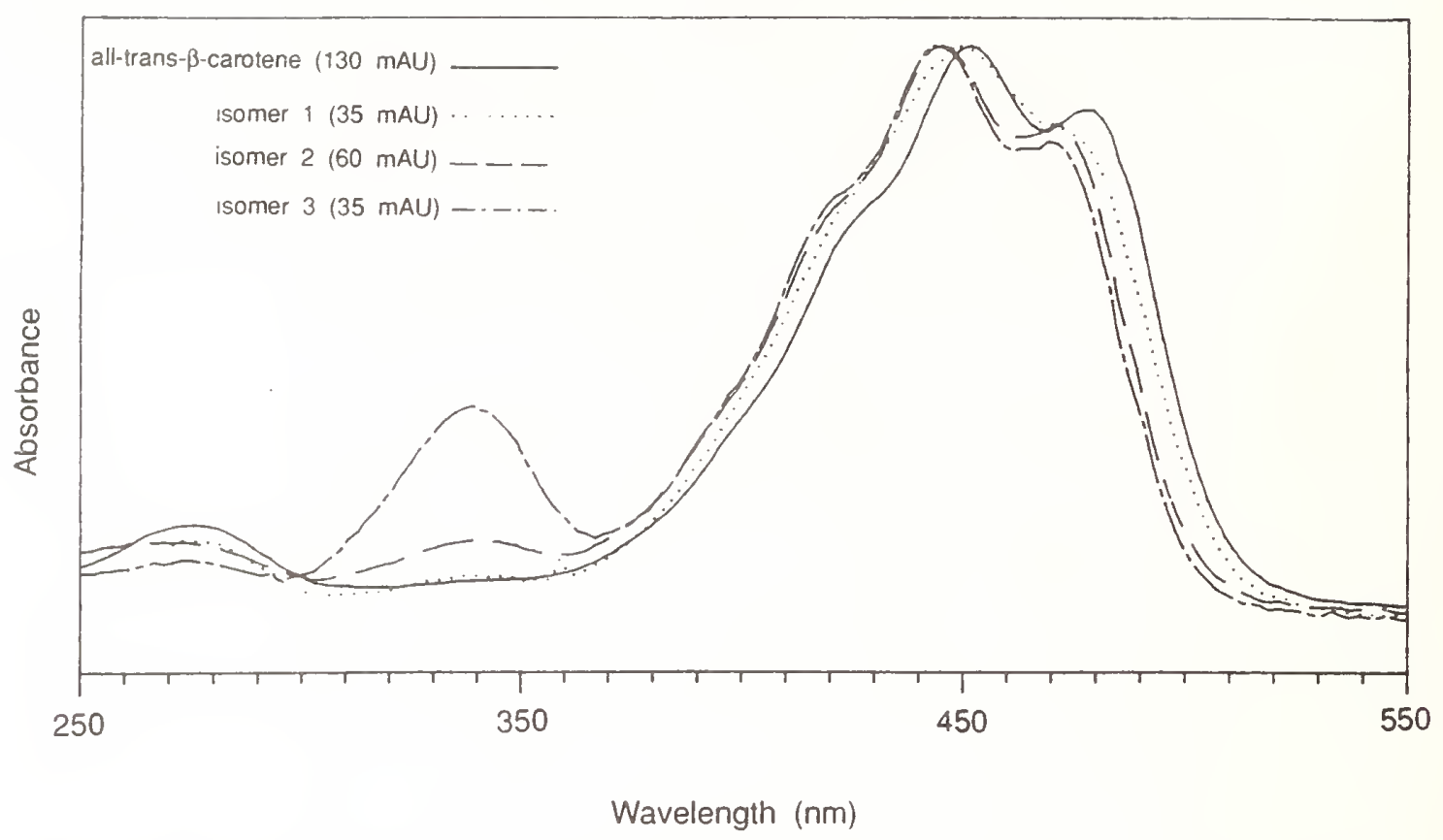

Fig. 4. Overlay of the absorption spectra of the major $\beta$-carotene isomers, present in the preparations investigated. Cis isomers are identified by order of elution from the LC column, as illustrated in Fig. 3.

cation is in agreement with a previous report describing the same algal source of $\beta$-carotene (Ben-Amotz et al., 1988). Spectra of the major $\beta$-carotene isomers in the carotene products are presented in Fig. 4. The $4 \mathrm{~nm}$ downward shift (hypsochromic shift) of the wavelength maxima in response to the introduction of a cis bond into the conjugated doublebond system is evident along with the characteristic 'cis' peak at 330$340 \mathrm{~nm}$. Based on Q-ratios and spectral maxima, peaks 1, 3 and 5 have been tentatively identified as 7 -cis- $\beta$-carotene, 13 -cis- $\beta$-carotene, and 9 , 13-di-cis- $\beta$-carotene, respectively. These identifications are termed tentative since spectral analysis alone is not a definitive technique for the identification of carotenoid isomers and coelution of isomers may exist. Studies are in progress to collect sufficient quantities of each isomer for more definitive identification by nuclear magnetic resonance spectroscopy.

The majority of methods used for carotenoid analysis lack the selectivity necessary to resolve very similar carotenoids, including the geometric isomers. In the present study, five LC columns were examined for their ability to separate the geometric isomers of $\beta$-carotene. Only the columns packed with wide-pore, polymerically synthesized $C_{18}$ were able to separate these isomers. We found that the recovery and selectivity of these columns was better using methanol-based mobile phases rather than acetonitrile-based. The $c$ is-component of all $\beta$-carotene preparations 
examined ranged from 10 to $67 \%$ of the total absorbance due to $\beta$-carotene. As illustrated here by $\beta$-carotene, accurate carotenoid measurements require the right selection of column and mobile phase; and due to the large degree of variability in the purity of commercial $\beta$-carotene preparations, special precautions must be taken when value assigning carotenoid calibration standards.

\section{ACKNOWLEDGEMENT}

This work was supported in part by research agreement Y01-CN-80655 from the Division of Cancer Prevention and Control, National Cancer Institute.

\section{REFERENCES}

Ben-Amotz, A., Lers, A. \& Avron, M. (1988). Stereoisomers of $\beta$-carotene and phytoene in the alga Dunaliella bardawil. Plant Physiol., 86, 1286-91.

Bushway, R. J. (1985). Separation of carotenoids in fruits and vegetables by high performance liquid chromatography. J. Liq. Chromatogr., 8, 1527-47.

Chandler, L. A. \& Schwartz, S. J. (1987). HPLC separation of cis-trans carotene isomers in fresh and processed fruits and vegetables. J. Food Sci., 52, 669-72.

Chandler, L. A. \& Schwartz, S. J. (1988). Isomerization and losses of trans$\beta$-carotene in sweet potatoes as affected by processing treatments. J. Agric. Food Chem., 36, 129-33.

Isler, O., Lindlar, H., Montavon, M., Rüegg, R. \& Zeller, P. (1956). Synthesen in der Carotinoid-Reihe. Die technische Synthese von $\beta$-Carotin. Helv. Chim. Acta, 39, 249-59.

MacCrehan, W. A. \& Schönberger, E. (1987). Determination of retinol, $\alpha$-tocopherol, and $\beta$-carotene in serum by liquid chromatography with absorbance and electrochemical detection. Clin. Chem., 33, 1585-92.

Mayne, S. T. \& Parker, R. S. (1988). cis-Canthaxanthin and other ca rotenoid-like compounds in canthaxanthin preparations. J. Agric. Food Chem., 36, 478-82.

Quackenbush, F. W. (1987). Reverse phase HPLC separation of cis- and transcarotenoids and its application to $\beta$-carotenes in food materials. J. Liq. Chromatogr., 10, 643-53.

Quackenbush, F.W.\& Smallidge, R. L. (1986). Nonaqueous reverse phase liquid chromatographic system for separation and quantitation of provitamins A. J. Assoc. Off. Anal. Chem., 69, 767-71.

Sander, L. C. \& Wise, S. A. (1984). Synthesis and characterization of polymeric C18 stationary phases for liquid chromatography. Anal. Chem., 56, 504-10.

Sander, L. C. \& Wise, S. A. (1986). Investigations of selectivity in RPLC of polycyclic aromatic hydrocarbons. In Advances in Chromatography, Vol. 25 eds J. C. Giddings, E. Grushka, J. Cazes \& P. R. Brown. Marcel Dekker, New York, pp. 139-218. 
Tsukida, K. \& Saiki, K. (1982). Separation and determination of cis/trans$\beta$-carotenes by high-performance liquid chromatography. J. Chromatogr., 245, 359-64.

Tsukida, K., Saiki, K. \& Sugiura, M. (1981). Structural elucidation of the main cis- $\beta$-carotenes. J. Nutr. Sci. Vitaminol., 27, 551-61.

Vecchi, M.. Englert, G., Maurer. R. \& Meduna, V. (1981). Trennung und charakterisierung von $\beta$-carotin-isomeren. Helv. Chim. Acta, 64, 2746-58.

Wise, S. A. \& Sander. L. C. (1985). Factors affecting the reversed-phase liquid chromatographic separation of polycyclic aromatic hydrocarbons. J. High Resol. Chromatogr. Chromatogr. Commun., 8, 248-55.

Wise, S. A., Sander, L. C. \& May. W. E. (1983). Modification of selectivity in reversed-phase liquid chromatography of polycyclic aromatic hydrocarbons using mixed stationary phases. J. Liq. Chromatogr., 6, 2709-21.

Zechmeister, L. (1962). Cis-Trans Isomeric Carotenoids, Vitamins A, and Arylpolyenes. Academic Press, New York. 


\title{
Relative Solubility, Stability, and Absorptivity of Lutein and $\beta$-Carotene in Organic Solvents
}

\author{
Neal E. Craft* \\ Organic Analytical Research Division, Chemical Science and Technology Laboratory, National Institute of \\ Standards and Technology, Gaithersburg, Maryland 20899 \\ Joseph H. Soares, Jr. \\ Nutritional Sciences Program, University of Maryland, College Park, Maryland 20742
}

\begin{abstract}
The relative solubility, stability, and absorptivity of lutein and $\beta$-carotene were determined in 18 organic solvents. The solubility of both carotenoids was greatest in tetrahydrofuran, while hexane exhibited the least solubility for lutein; methanol and acetonitrile exhibited the least solubility for $\beta$-carotene. Stability was monitored for 10 days at room temperature by measuring absorbance changes at the wavelength maximum. In the majority of the solvents, initial absorbance decreased by less than $10 \%$ during the 10-day period. Degradation was greatest for both carotenoids in cyclohezanone. The relative absorptivities were determined by calculating the carotenoid concentration in a reference solvent using a reference absorptivity, and then Beer's Law was applied to the measured absorbance of the same carotenoid concentration in other organic solvents. Absorbance maxima and relative absorptivities were in good agreement with available literature values.
\end{abstract}

Interest in carotenoids has increased during the past decade. Carotenoids are not only natural pigments and vitamin A precursors but have been proposed as cancer prevention agents, ulcer inhibitors, life extenders, and heart attack inhibitors (Peto et al., 1981; Colditz et al., 1985; Mozsik et al., 1984; Cutler, 1984; Gaziano et al., 1990). Unfortunately, physical information about these compounds in organic solvents is limited. The wavelength maxima and absorptivity of carotenoids change with the nature of the solvent in which they are dissolved. For example, the visible spectrum of $\beta$-carotene in ethanol has little fine structure with absorbance maxima at 453 and $480 \mathrm{~nm}$, while the visible spectrum in carbon disulfide has more fine structure and exhibits maxima at 484 and $512 \mathrm{~nm}$ (Davies, 1976). The molar absorptivities of $\beta$-carotene at $\lambda_{\max }$ in these two solvents are 140700 and $107800 \mathrm{~L} \mathrm{~mol}^{-1} \mathrm{~cm}^{-1}$, respectively (Davies, 1976). In the past, absorbance maxima have been compiled for carotenoids in several solvents (Davies, 1976; De Ritter and Purcell, 1981), but such tables supply limited absorptivities, and frequently the maxima in a given solvent vary by several nanometers depending on the source of information. These tables also provide no information regarding the solubility and stability of the carotenoids in the solvents. The lack of information about carotenoid solubilities and molar absorptivities in a variety of organic solvents increases the difficulty associated with developing analytical methods for carotenoid research. Such practical information is important for the selection of solvents for use in sample preparation and liquid chromatography (LC) mobile phases and also for the identification and quantification of carotenoids in diverse LC mobile phases.

The two most prominent cyclized carotenoids in human serum and foods are lutein $\left(\beta, \epsilon\right.$-carotene- $3,3^{\prime}$-diol) and $\beta$ carotene ( $\beta, \beta$-carotene) (Bieri et al., 1985; Khachik et al., 1986). Not only are they the most prominent, but they also span a wide polarity range and are representative of the $\beta, \epsilon$-and the $\beta, \beta$-carotenoids, respectively. Herein we describe the determination of the relative solubility,

* Author to whom correspondence should be addressed. stability, and absorptivity of these two biologically important carotenoids in various organic solvents.

\section{MATERIALS AND METHODS}

Reagents. Crystalline $\beta$-carotene (type I, Sigma Chemical Co., St. Louis, MO) and lutein (provided as a gift by Kemin Industries, Des Moines, IA) used throughout the study were assessed by spectrophotometric and liquid chromatographic techniques to be greater than $90 \%$ trans- $\beta$-carotene and $90 \%$ trans-lutein, respectively. The sources, descriptions, and lot numbers of the solvents used are listed in Table 1 .

Equipment. Spectral measurements were made using a photodiode array scanning spectrometer (H-P 8450, Hewlett-Packard, Palo Alto, CA). The spectrophotometer provided a 1-nm spectral band pass from 200 to $400 \mathrm{~nm}$ and a 2 -nm spectral band pass from 400 to $800 \mathrm{~nm}$. Wavelength accuracy was checked using a holmium oxide glass filter and found to be correct at the 279-,361-, 460-, and 536-nm absorption maxima. Solutions were dispensed with calibrated pipets or gas-tight syringes. Carotenoid purity was determined as previously described (Craft et al., 1991).

Relative Solubility of Lutein and $\beta$-Carotene in Organic Solvents. Approximately $10 \mathrm{mg}$ of lutein or $\beta$-carotene was added to $3 \mathrm{~mL}$ of each of the solvents listed in Table I. Vials were ultrasonically agitated for $5 \mathrm{~min}$. If a clear solution with no residual crystals resulted, additional carotenoid was added until crystalline material remained undissolved. Each solution was then filtered through a $0.2-\mu \mathrm{m}$ membrane, and appropriate dilutions were made until the absorbance at the wavelength maximum was between 0.5 and 1.0 absorbance unit at ambient temperature. The background absorbance of each solution was subtracted using the appropriate solvent containing no carotenoid. Carotenoid concentration was calculated using Beer's law and the relative absorptivities determined below (Determination of Relative Absorptivity). Measurements were performed in triplicate and the calculation used is

(absorbance, at $\lambda_{\max }$ )(dilution factor)/molar absorptivity,

where the subscript $\mathrm{s}$ is a given solvent. The measured values were rounded to one significant figure since this experiment was not designed to determine absolute solubility but rather to indicate solubility relative to other solvents.

Determination of Relative Absorptivity. Concentrated solutions (approximately $3 \mathrm{~g} / \mathrm{L}$ ) of lutein and $\beta$-carotene were prepared in tetrahydrofuran (THF) containing butylated hy- 
Table I. List of Solvents, Sources, and Lot Numbers

\begin{tabular}{|c|c|c|c|}
\hline solvent & source, grade & lot & safety hazards ${ }^{\circ}$ \\
\hline acetone & Mallinckrodt, SpectAR & 2438 & $1,2,5$ \\
\hline acetonitrile & J. T. Baker, HPLC & C28108 & $2,3,5$ \\
\hline benzene & J.T. Baker, Photrex & $\mathrm{C} 14603$ & 3,5 \\
\hline chloroform & EM Science, Omnisolve & 5102 & 3 \\
\hline cyclohexane & EM Science, Omnisolve & 6041 & $1,2,5$ \\
\hline cyclohexanone & Kasai, GR & FAV01 & 1,2 \\
\hline dichloromethane & J. T. Baker, HPLC & D25082, D18131 & 2,3 \\
\hline dimethylformemide (DMF) & Burdick and Jackson, HPLC & AK285 & 1,2 \\
\hline dimethyl sulforide (DMSO) & Mallinckrodt, SpectAR & KMCD & $1,2,4$ \\
\hline ethanol, absolute & Warner-Graham & & 1,5 \\
\hline ethyl acetate & EM Science, Omnisolve & 7278 & L, 2,5 \\
\hline ethyl ether & EM Science, GR & 9130 & $1,2,4,5$ \\
\hline herane & J. T. Baker, HPLC & D33095 & $1,2,5$ \\
\hline 2-propanol & Mallinckrodt, AR & $3037 \mathrm{KDEV}, 3035 \mathrm{KCAY}$ & 1,5 \\
\hline methanol & EM Science, Omnisolve & 8352 & 3,5 \\
\hline methyl tert-butyl ether (MTBE) & EM Science, reagent & $11 \mathrm{P} 23$ & 2,5 \\
\hline tetrahydrofuran (THF) + BHT & J. T. Baker, HPLC & $\mathrm{C} 24654$ & 2,5 \\
\hline toluene & Burdick and Jackson, HPLC & AK80 & $1,2,5$ \\
\hline
\end{tabular}

a 1 , harmful when entering the body; 2 , irritant to skin, eyes, and respiratory organs; 3 , toxic (harmf ul if inhaled, ingested, or absorbed through the skin); 4 , explosive; 5 , flammable.

Table II. Relative Solubility and Absorptivity of Lutein and $\beta$-Carotene in Organic Solvents

\begin{tabular}{|c|c|c|c|c|c|c|c|c|}
\hline \multirow[b]{2}{*}{ solvent } & \multicolumn{4}{|c|}{ lutein } & \multicolumn{4}{|c|}{$\beta$-carotene } \\
\hline & $\begin{array}{l}\text { solubility, } \\
\mathrm{mg} / \mathrm{L}\end{array}$ & $\lambda_{\max },{ }^{a} \mathrm{~nm}$ & $\begin{array}{c}\text { absorptivity } \\
E^{1 \%}, \mathrm{~cm}^{-1}\end{array}$ & $\begin{array}{l}\text { molar absorptivity, } \\
\qquad \mathrm{L} \mathrm{mol}^{-1} \mathrm{~cm}^{-1}\end{array}$ & $\begin{array}{l}\text { solubility, } \\
\mathrm{mg} / \mathrm{L}\end{array}$ & $\lambda_{\max },{ }^{a} \mathrm{~nm}$ & $\begin{array}{c}\text { absorptivity } \\
E^{1 \%}, \mathrm{~cm}^{-1}\end{array}$ & $\begin{array}{l}\text { molar absorptivity, } \\
\qquad \mathrm{L} \mathrm{mol}^{-1} \mathrm{~cm}^{-1}\end{array}$ \\
\hline acetone & 800 & 446 & 2540 & 144500 & 200 & $452(452)$ & 2559 & 137400 \\
\hline acetonitrile & 100 & 446 & 2559 & 145600 & 10 & 452 & 2540 & 136400 \\
\hline benzene & 600 & $456(458)$ & 2350 & $133700(127200)$ & 4000 & $462(462)$ & $230 \iota_{\mathrm{t}}$ & $124000(125500)$ \\
\hline chloroform & 6000 & $454(458)$ & 2369 & 134800 & 2000 & $462(461)$ & 2330 & $125100(128600)$ \\
\hline cycloherane & 50 & 448 & 2520 & 143400 & 2000 & $454(457)$ & $25^{\prime} / 8$ & $134700(134500)$ \\
\hline cyclohexanone & 4000 & 454 & 2359 & 134200 & 2000 & 462 & 2359 & 126700 \\
\hline dichloromethane & 800 & 452 & 2320 & 132000 & 6000 & 460 & 2369 & 127200 \\
\hline DMF & 1000 & 454 & 2390 & 136000 & 200 & 460 & 2389 & 128300 \\
\hline DMSO & 1000 & 460 & 2369 & 134800 & 30 & 466 & 2259 & 121300 \\
\hline ethanol & 300 & $444(445)$ & 2550 & $145100^{b}$ & 30 & $450(449)$ & 2529 & $135800(140700)$ \\
\hline ethyl acetate & 800 & 446 & 2529 & 143900 & 500 & 452 & 2520 & 135300 \\
\hline ethyl ether & 2000 & 444 & 2629 & 149600 & 1000 & 448 & 2659 & 142800 \\
\hline herane & 20 & $444(445)$ & 2589 & 147300 & 600 & $448(453,450)$ & 2592 & $139200^{c}$ \\
\hline 2-propanol & 400 & 444 & 2599 & 147900 & 40 & 450 & 2508 & 134700 \\
\hline methanol & 200 & $442(444)$ & 2629 & 149600 & 10 & 450 & 2540 & 136400 \\
\hline MTBE & 2000 & 444 & 2589 & 147300 & 1000 & 450 & 2588 & 139000 \\
\hline THF & 8000 & 450 & 2469 & 140500 & 10000 & 456 & 2399 & 128800 \\
\hline toluene & 500 & 456 & 2290 & 130300 & 4000 & $462(463)$ & 2270 & 121900 \\
\hline
\end{tabular}

a Calculated molar absorptivities and $\lambda_{\max }$ in parentheses are taken from Davies (1976). ${ }^{b}$ Reference absorptivity for lutein. ${ }^{c}$ Reference absorptivity for $\beta$-carotene.

droxytoluene $(\mathrm{BHT})$ as an antioxidant and filtered through $0.2-$ $\mu \mathrm{m}$ membranes. To $30 \mathrm{~mL}$ of each of the solvents listed in Table I was added $30 \mu \mathrm{L}$ of concentrated lutein or $\beta$-carotene in THF. We were cautious to work well within the carotenoid solubility limits (determined above) of the solvents being examined to avoid precipitation of the carotenoid compounds. Sealed vials were ultrasonically agitated for $3 \mathrm{~min}$ to assure dissolution. Spectrophotometric scans were performed from 250 to $550 \mathrm{~nm}$, and absorbance at the wavelength maximum was determined. The concentrations of the lutein and $\beta$-carotene solutions were determined using the most widely accepted mular absorptivity for lutein in ethanol and $\beta$-carotene in hezane (145 100 and 139200 $\mathrm{L} \mathrm{mol}^{-1} \mathrm{~cm}^{-1}$, respectively) (Davies, 1976). Relative absorptivities in the different solvents were determined on the basis of the calculated concentrations of lutein and $\beta$-carotene determined in ethanol and hezane, respectively, and the absorbance of the carotenoid solutions at the wavelength maximum in a given solvent using Beer's law. Absorbance measurements were performed in triplicate, and the combined error (mean standard deviation of absorbance measurements and estimated limits of bias) associated with the measurements was approzimately $1 \%$.

Lutein and $\beta$-Carotene Stability. The solutions prepared under Determination of Relative Absorptivity were stored in amber glass vials with Teflon-lined screw caps at room temperature. The UV-vis absorbance spectrum from 250 to $550 \mathrm{~nm}$ was monitored over a 10-day period. Decreases in absorbance and shifts in wavelength maxima were indicators of carotenoid degradation. Degradation is reported as percent of initial absorbance at $\lambda_{\max }$.

\section{RESULTS AND DISCUSSION}

Both carotenoids tested were most soluble in THF (Table II). $\beta$-Carotene was least soluble in methanol and acetonitrile, while lutein was least soluble in herane. Many existing extraction techniques partition carotenoids into hexane or petroleum ether from aqueous alcohol or acetone (Bieri et al., 1985; De Ritter and Purcell, 1981; Simpson et al., 1985). Given the poor solubility of dihydrozy and more polar carotenoids in hexane, this may lead to losses. Diethyl ether has also been used to partition carotenoids from aqueous/polar organic mixtures (Britton, 1985; De Ritter and Purcell, 1981; Rodriquez-Amaya, 1989); on the basis of the solubilities listed in Table II, this may present a more effective approach. One possible disadvantage is the solubility of fatty acid soaps in ether which must be thoroughly removed with water (Britton, 1985). Although THF is subject to peroxide formation, it has found increased use (Bureau and Bushway, 1986; Khachik et al., 1986; Peng et al., 1987) for carotenoid extractions due to the high solubility of a wide polarity range of carotenoids. We are unaware of published absorptivities for carotenoids in THF. This lack of information may hamper its use as a solvent and result in the introduction of errors associated with evaporation and solvent-transfer steps due to the use of less appropriate solvents with published absorptivities. The information listed in Table II should prove 


\begin{tabular}{|c|c|c|c|c|c|c|c|c|c|c|c|c|}
\hline \multirow[b]{3}{*}{ solvent } & \multicolumn{6}{|c|}{${ }_{c}$ of initial absorbance of lutein at $\lambda_{\max }$} & \multicolumn{6}{|c|}{$r_{0}$ of initial absorbance of $\beta$-carotene at $\lambda_{\max }$} \\
\hline & \multicolumn{4}{|c|}{ time. days } & \multirow{2}{*}{$\begin{array}{c}\lambda_{\max } \\
\text { shift, }{ }^{a} \mathrm{~nm}\end{array}$} & \multirow{2}{*}{$\begin{array}{c}\text { cis } \\
\text { peak }^{b}\end{array}$} & \multicolumn{4}{|c|}{ time, days } & \multirow{2}{*}{$\begin{array}{c}\lambda_{\operatorname{mex}} \\
\text { shift, }{ }^{a} \mathrm{~nm}\end{array}$} & \multirow{2}{*}{$\begin{array}{c}\text { cis } \\
\text { peak }^{b}\end{array}$} \\
\hline & 1 & 3 & 6 & 10 & & & 1 & 3 & 6 & 10 & & \\
\hline acetone & 99 & 96 & 95 & 95 & 0 & + & 98 & 96 & 96 & 93 & 0 & + \\
\hline acetonitrile & 98 & 97 & 94 & 94 & -2 & + & 99 & 96 & 93 & 92 & 0 & + \\
\hline benzene & 100 & 99 & 100 & 97 & 0 & - & 87 & 77 & 71 & 67 & 0 & - \\
\hline chloroform & 97 & 96 & 93 & 90 & -2 & + & 97 & 91 & 92 & 91 & -2 & + \\
\hline cyclohexane & 100 & 100 & 98 & 99 & 0 & - & 98 & 98 & 93 & 91 & 0 & + \\
\hline cyclohexanone & 88 & 70 & 49 & 37 & -2 & +++ & 86 & 67 & 45 & 32 & -4 & +++ \\
\hline dichloromethane & 95 & 91 & 88 & 83 & 0 & - & 77 & 59 & 47 & 34 & -30 & + \\
\hline DMF & 100 & 100 & 97 & 97 & 0 & - & 96 & 94 & 93 & 90 & 0 & - \\
\hline DMSO & 100 & 99 & 99 & 57 & 0 & - & 94 & 90 & 86 & 80 & 0 & - \\
\hline ethanol & 96 & 96 & 93 & 91 & 0 & + & 98 & 94 & 92 & 91 & -2 & + \\
\hline ethyl acetate & 98 & 97 & 95 & 96 & 0 & - & 99 & 97 & 96 & 95 & 0 & - \\
\hline ethyl ether & 96 & 88 & 79 & 65 & -2 & ++ & 94 & 78 & 70 & 69 & -2 & + \\
\hline hexane & 99 & 100 & 100 & 98 & 0 & - & 96 & 95 & 94 & 92 & 0 & + \\
\hline 2-propanol & 99 & 99 & 99 & 95 & 0 & - & 97 & 94 & 94 & 89 & 0 & - \\
\hline methanol & 97 & 95 & 95 & 90 & -2 & ++ & 97 & 92 & 89 & 88 & -4 & ++ \\
\hline MTBE & 97 & 90 & 82 & 76 & 0 & ++ & 96 & 89 & 82 & 74 & -2 & ++ \\
\hline $\mathrm{THF}+\mathrm{BHT}$ & 100 & 100 & 100 & 99 & 0 & - & 99 & 98 & 99 & 97 & 0 & - \\
\hline toluene & 99 & 98 & 100 & 97 & 0 & - & 90 & 80 & 76 & 71 & 0 & - \\
\hline
\end{tabular}

${ }^{a}$ Indicates direction and amount of spectral shift at day $10 .{ }^{b}$ Indicates presence/absence of cis peak. Number of + 's indicates intensity of cis peak.

valuable in the selection of solvents employed for carotenoid extractions and dissolutions.

The wavelength maximum for $\beta$-carotene in the various solvents ranged from 448 to $466 \mathrm{~nm}$. The $\lambda_{\max }$ in hexane was $448 \mathrm{~nm}$, which is $4-5 \mathrm{~nm}$ below the referenced value (Davies, 1976), although the same reference lists other citations reporting $\lambda_{\max }$ in hexane at $449 \mathrm{~nm}$ but without an absorptivity. The difference in the reference $\lambda_{\max }$ and the $\lambda_{\max }$ obtained in this work can be attributed to aromatic contaminants in some solvents, the presence of cis isomers in the $\beta$-carotene employed, and differences in the wavelength calibration or spectral band pass of the spectrophotometers used for the measurements. For the purposes of this work, the maximum absorbance was used to determine relative solubilities and absorptivities. The wavelength maximum for lutein ranged from 442 to 460 $\mathrm{nm}$. The $\lambda_{\max }$ of lutein in ethanol was within $1 \mathrm{~nm}$ of the reported value of $445 \mathrm{~nm}$ (Davies, 1976). It may be that, in the lutein used, the presence of $\sim 6 \%$ zeaxanthin, which has a higher $\lambda_{\max }$, offset the wavelength lowering due to cis isomers or differences in wavelength calibration. The spectrophotometer used for this work was limited to a 2 -nm spectral band pass in the visible region. These two observations may explain why the measured $\lambda_{\max }$ values were consistently 1-2 nm lower than reported values.

Because the absorptivity and $\lambda_{\max }$ of carotenoids vary in different solvents and these values are only published for a few solvents, relative absorptivities and $\lambda_{\max }$ were determined for lutein and $\beta$-carotene in the solvents listed in Table I. These values, given in Table II, were used to calculate the solubility of the carotenoids, also reported in Table II. Relative absorptivity values listed in Table II are in good agreement with previously published values (Davies, 1976; De Ritter and Purcell, 1981). The primary advantage of the determination of the spectral maxima and absorptivities is that carotenoid concentrations can be determined directly in a wide range of solvents. In addition, data obtained using diode array detectors in conjunction with LC can be better interpreted when the wavelength shifts that occur in different solverts are known.

Finally, the stability of these two carotenoids in the various solvents was monitored spectrophotometrically over a period of 10 days. Carotenoid degradation was accompanied by decreases in the absorbance and, in some cases, a downward shift in the $\lambda_{\max }$ (Table III). We are aware that some degradation products (e.g., geometric isomers and carotenals) contribute to the absorbance in the visible region; however, all degradation products exhibit lower absorptivity at the wavelength maximum of the parent compound. This also implies that changes in absorbance are not necessarily proportional to the concentration of lutein or $\beta$-carotene in the solution. The definitive measure of degradation would have been to monitor the trans isomer of both carotenoids by HPLC; however, while attempting to do this, we encountered technical difficulties. First, it was not possible to make all of the measurements by HPLC at the appointed times without staggering the experiments; second, few of the solvents could be injected directly into the HPLC system; and third, complete redissolution of carotenoids was questionable if a solvent evaporation was included. For these reasons we opted to record the UV-vis spectra to monitor major changes in the analytes. When the absorbance expressed as percent of initial absorbance at $\lambda_{\max }$ was plotted against time, the degradation function was similar in all solvents but proceeded at different rates (Figure 1). Stability was poorest for both lutein and $\beta$ carotene in cyclohexanone, retaining only $37 \%$ and $32 \%$, respectively, of their initial absorbance by day 10 . The degradation of $\beta$-carotene in cyclohezanone was followed closely by degradation in dichloromethane with $\sim 34 \%$ absorbance remaining at day 10 . In general, the rate of lutein degradation was slower than $\beta$-carotene degradation as illustrated by the curves shown in Figure 1 representing the average rate of degradation in all solvents. Even on day 10 , the carotenoid absorbance in most solvents was clustered above $90 \%$ of the initial absorbance. The conditions incorporated were selected to exacerbate the degradation process so that stability/instability would be evident. In a laboratory setting, greater efforts would be made to stabilize carotenoid solutions, e.g., by the incorporation of antioxidants and use of lower storage temperatures.

Little information of this type has been reported previously. In comprehensive reviews (Davies, 1976; De Ritter and Purcell, 1981), references to factors important to solvent selection are mentioned, but specific information about the influence of solvents on carotenoid stability is lacking. Information presented in Table III should be used for comparative purposes since stability is also dependent on solvent supplier and lot number. The solvents 
LUTEIN DEGRADATION IN ORGANIC SOLVENTS

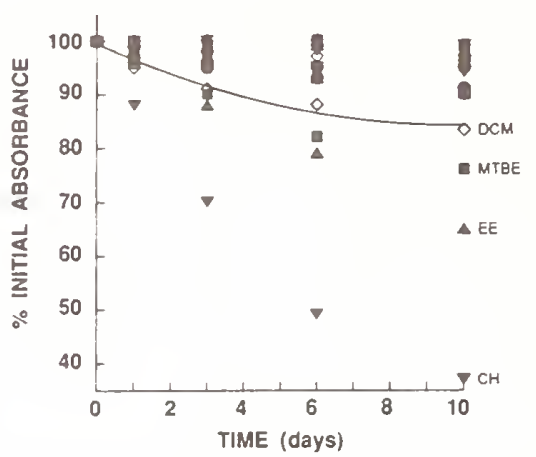

B-CAROTENE DEGRADATION IN ORGANIC SOLVENTS

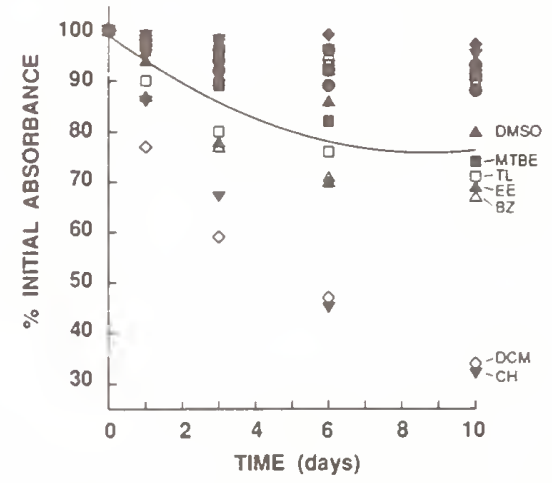

Figure 1. Percent initial absorbance at $\lambda_{\max }$ of lutein and $\beta$ carotene monitored in 18 organic solvents over a period of 10 days at ambient temperature. Solid line represents the average rate of degradation in all solvents. Actual values for a given solvent are listed in Table III. A specific symbol is used to illustrate the degradation in a given solvent; however, duplicates of most symbols were necessary to account for all solvents. As such, legends are not given for each symbol; however, solvents with less than $85 \%$ of initial absorbance at day 10 are indicated by solvent abbreviations. Abbreviations: $\mathrm{BZ}$, benzene; $\mathrm{CH}$, cyclohexanone; DCM, dichloromethane; EE, ethyl ether; MTBE, methyl tert-butyl ether; TL, toluene.

used may or may not be representative of current lots from a given supplier. No attempt was made to sample various sources of each solvent, and no additional antioxidants were added to the solvents used. However, our experience with 2-propanol and dichloromethane indicates that the source and lot of solvent used substantially influence the stability of carotenoids in solution.

While developing methods for the extraction and determination of carotenoids, we found that critical information was frequently missing from tabulated data and found it necessary to fill in some gaps. The information provided in this paper supplements published absorptivities and $\lambda_{\max }$ values for solvents for which information is currently unavailable. This information should also aid in the selection of solvents to be used for carotenoid research by giving an indication of stability and solubility of two carotenoids, which vary greatly in polarity. Finally, since the chromophore is not strongly influenced by the presence of hydroxyl groups outside the conjugated double bond system, the molar absorptivity values for lutein can be used for estimating concentration values of other $\beta, \epsilon-$ carotenoids such as $\alpha$-cryptozanthin and $\alpha$-carotene; the molar absorptivity values for $\beta, \beta$-carotene can be used for $\beta$-cryptoxanthin and zeaxanthin.

\section{ACKNOWLEDGMENT}

We are grateful to Chris Nelson and Kemin Industries for the generous gift of lutein. The work is taken in part from a dissertation submitted to the Graduate School, University of Maryland, by N.E.C. in partial fulfillment of the requirement for the Ph.D. degree in Nutritional Sciences.

\section{LITERATURE CITED}

Bieri, J.G.; Brown, E. D.; Smith, J. C. Determination of individual carotenoids in human plasma by high performance liquid chromatography. J. Liq. Chromatogr. 1985, 8, 473-484.

Britton, G. General Carotenoid Methods. Methods Enzymol. $1985,111,113-149$.

Bureau, J. L.; Bushway, R. J. HPLC determination of carotenoids in fruits and vegetables in the United States. J. Food Sci. 1986, 51, 128-130.

Colditz, G. A.; Branch, L. G.; Lipnick, R. J.; Willett, W. C.; Rosner, B.; Posner, B. M.; Hennekens, C. H. Increased green and yellow vegetable intake and lowered cancer deaths in an elderly population. Am. J. Clin. Nutr. 1985, 41, 32-36.

Craft, N. E.; Sander, L. C.; Pierson, H. F. Separation and Relative Distribution of all-trans- $\beta$-Carotene and its cis-Isomers in $\beta$ Carotene Preparations. J.Micronutr. Anal. 1991,8, 209-221.

Cutler, R. G. Carotenoids and retinol: Their possible importance in determining longevity of primate species. Proc. Natl. Acad. Sci. U.S.A. 1984, 87, 7627-7631.

Davies, B. H. Carotenoids. In Chemistry and Biochemistry of Plant Pigments, 2nd ed.; Goodwin, T. W., Ed.; Academic Press: London, 1976; Vol. 2, pp 38-165.

De Ritter, E.; Purcell, A. E. Carotenoid Analytical Methods. In Carotenoids as Colorants and Vitamin A Precursors; Bauernfeind, J. C., Ed.; Academic Press: London, 1981; pp 815-923.

Gaziano, J. M.; Manson, J. E.; Ridker, P. M.; Buring, J. E.; Hennekens, C. H., $\beta$-Carotene and Heart Disease. Circulation 1990, 82, 796 (Abstract).

Khachik, F.; Beecher, G. R.; Whittaker, N. F. Separation, identification, and quantification of the major carotenoid and chlorophyll constituents in extracts of several green vegetables by liquid chromatography. J. Agric. Food Chem. 1986, 34, $603-616$.

Mozsik, G.; Javor, T.; Toth, G.; Zsoldos, T.; Tigyi, A. Interrelationships between the Gastric Cytoprotective Effects of Vitamin $A$ and $\beta$-Carotene and the Gastric Mucosal Superoxide Dismutase Activity in Rats. Acta Phys. Hung. 1984, 64, 315-318.

Peng, Y.-M.; Xu, M.-J.; Alberts, D. S. Analysis and stability of retinol in plasma. J. Natl. Cancer Inst. 1987, 78, 95-99.

Peto, R.; Doll, R.; Buckley, J. D.; Sporn, M. B. Can dietary $\beta$ carotene materially reduce human cancer rates? Nature 1981 , 290, 201-208.

Rodriquez-Amaya, D. Critical Review of Provitamin A Determination in Plant Foods. J. Micronutr. Anal. 1989, 5, 191225.

Simpson, K. L.; Tsou, S. C. S.; Chichester, C. O. Carotenes. In Methods of Vitamin Assay, 4th ed.; Augustin, J., Klein, B. P., Becker, D. A., Venugopal, P. B., Eds.; Wiley: New York, 1985; pp 185-220.

Received for review June 27, 1991. Accepted December 2, 1991. Certain commercial equipment, instruments, or materials are identified in this paper to specify adequately the experimental procedure. Such identification does not imply recommendation of endorsement by NIST, nor does it imply that the equipment or materials identified are necessarily the best available for the purpose.

Registry No. Lutein, 127-40-2; $\beta$-carotene, 7235-40-7; acetone, 67-64-1; acetonitrile, 75-05-8; benzene, 71-43-2; chloroform, 6766-3; cyclohexane, 110-82-7; cyclohexanone, 108-94-1; dichloromethane, 75-09-2; dimethylformamide, 68-12-2; dimethyl sulfoxide, 67-68-5; ethanol, 64-17-5; ethyl acetate, 141-78-6; ethyl ether, 60-29-7; hexane, 110-54-3; 2-propanol, 67-63-0; methanol, 67-56-1; methyl tert-butyl ether, 1634-04-4; tetrahydrofuran, 10999-9; toluene, 108-88-3. 


\title{
Optimization of an isocratic high-performance liquid chromatographic separation of carotenoids
}

\author{
Neal E. Craft* and Stephen A. Wise \\ Organic Analyical Research Division. Chemical Science and Technology Laboratory, National Institute of Standards and Technology, \\ Gaithersburg, MD 20899 (USA)
}

Joseph H. Soares, Jr.

Nutritional Sciences Department, University of Maryland. College Park, MD 20742 (USA)

(First received June 28th, 1991: revised manuscript received September 10th, 1991)

\begin{abstract}
Using a polymeric $\mathrm{C}_{18}$ high-performance liquid chromatographic (HPLC) column, which demonstrated excellent separation selectivity toward carotenoid compounds in an earlier column evaluation, the effects of mobile phase modifier, modifier concentration, and column temperature were investigated. A seven-component carotenoid mixture was used to monitor changes in separation selectivity in response to variations in HPLC conditions. Both acetonitrile and tetrahydrofuran (THF) improved the resolution of echinenone and $x$-carotene: THF was selected for use as a modifier due to its solvating properties. At concentrations greater than $6 \%$ THF, the resolution of lutein and zeaxanthin deteriorated significantly. Temperature was varied from 15 to $35^{\circ} \mathrm{C}$ in $5^{\circ} \mathrm{C}$ increments. Resolution of lutein/zeaxanthin and $\beta$-cartone/lycopene were better at lower temperatures while echinenone/ $\alpha$-carotene separation improved as temperature increased. An acceptable separation of all seven carotenoids was achieved at $20^{\circ} \mathrm{C}$ using $5 \%$ THF as a mobile phase modifier. Method applicability is demonstrated for serum and food carotenoids.
\end{abstract}

\section{INTRODUCTION}

During the past decade carotenoid pigments have become highly publicized compounds due to their potential roles in cancer prevention [1-4], as antioxidants [5], and in the reduction of atherogenesis [6]. As a result, several publications have reported highperformance liquid chromatographic (HPLC) methods for the determination of carotenoids [712], but no publications have reported the influence of individual HPLC parameters on the separation of multiple carotenoids. Few laboratories have the time or the resources to evaluate a large sample of HPLC columns during analytical method development. Therefore. most "new" methods of separation are only slight adjustments in mobile phase composition. Recently, Epler et al. [13] evaluated 65 HPLC columns under standardized conditions with respect to carotenoid separation and recovery. In this study they observed, as others have reported $[11,12]$ that polymeric $C_{18}$ phases exhibited excellent selectivity for structurally similar carotenoids, whereas monomeric $\mathrm{C}_{18}$ phases, which represent the majority of the commercial $\mathrm{C}_{18}$ columns available, exhibited less selectivity for structurally similar carotenoids. Extensive investigations of the stationary phase characteristics responsible for differences in selectivity between monomeric and polymeric $C_{18}$ phases for the separation of polycyclic aromatic hydrocarbon isomers have been reported by Sander and Wise [14]. Many of these same stationary phase characteristics influence the separation of carotenoids.

This report describes the optimization of an isocratic carotenoid HPLC separation using one HPLC column that exhibited very good separation 
of the carotenoid mixture during the column evaluation study. The influence of multiple solvent modifiers and temperature on the separation of a carotenoid mixture was examined.

\section{EXPERIMENTAL}

\section{Reagents}

An ethanolic solution containing between 0.2 and $1.0 \mu \mathrm{g} / \mathrm{ml}$ of lutein $\left[\left(3 R, 3^{\prime} R, 6^{\prime} R\right)-\beta, \varepsilon\right.$-carotene- $3,3^{\prime}-$ diol, Kemin Industries, Des Moines, IA, USA], zeaxanthin $\left[\left(3 R, 3^{\prime} R\right)-\beta, \beta\right.$-carotene- $3,3^{\prime}$-diol], $\beta$-cryptoxanthin [( $3 R)-\beta, \beta$-caroten-3-ol, Atomergic Chemetals, Farmingdale, NY, USA], echinenone $(\beta, \beta$-caroten-4-one) (Hoffmann-La Roche, Nutley, NJ, USA), lycopene $(\psi, \psi$-carotene, extracted from tomato paste $), \alpha$-carotene $\left[\left(6^{\prime} R\right)-\beta, \varepsilon\right.$-carotene $]$, and $\beta$-carotene $(\beta, \beta$-carotene, Sigma, St. Louis, MO, USA) was prepared to monitor column separation selectivity. The following solvents were used in the mobile phase mixtures: acetone, acetonitrile, chloroform, diethyl ether, ethyl acetate, hexane, methanol, methylene chloride, tetrahydrofuran (THF) containing butylated hydroxytoluene (BHT) and toluene. All solvents were HPLC grade or equivalent and were used without further treatment.

\section{HPLC}

The HPLC system consisted of: a dual piston. quaternary low-pressure gradient solvent delivery system equipped with helium sparging and column temperature control; a high sensitivity, programmable, rapid scanning, UV-visible detector fitted with both deuterium and tungsten lamps and an 8- $\mu$ l flow cell; and manual and automatic injection valves. Carotenoids were monitored at $445 \mathrm{~nm}$ and a computer-controlled data system was used to process data permitting review and manipulation of peak integration. The HPLC column contained 300 $\AA$ pore diameter, $5 \mu \mathrm{m}$ particle size, silica polymerically modified with $\mathrm{C}_{18}$ (Vydac 201TP, $25 \times 4.6$ cm I.D. Separations Group, Hesperia, CA, USA). Other columns with similar chemistries could be substituted with minor modifications in mobile phase composition [13]. A $0.2 \mu \mathrm{m}$ inline filter was placed between the pump and injection valve to trap particles resulting from piston seal wear. All stainless steel frits between the injection valve and the detector were replaced with biocompatible ce- ramic frits to minimize carotenoid precipitation and degradation $[13,15]$. A guard column containing $5-\mu \mathrm{m}_{18}$ material similar to the analytical column was directly attached to the analytical column inlet to remove particulate material and protect the column.

A methanol-based mobile phase was modified with 5 to $10 \%$ of the solvents listed under Reagents to determine the influence of each solvent on the separation of the carotenoid test mixture. After the modifier yielding the best selectivity was selected (THF), incremental portions were added to methanol to achieve the best separation of all seven carotenoids as determined by the shortest analysis time without compromising the resolution of all carotenoid peaks.

Column temperature was varied in $5^{\circ} \mathrm{C}$ increments between 15 and $35^{\circ} \mathrm{C}$ to investigate the influence of temperature on the separation of the carotenoid mixture. Temperature was regulated by the thermostatically controlled column oven on the HPLC system. The $15^{\circ} \mathrm{C}$ column temperature was achieved by placing a beaker of dry ice at the forced air inlet to reduce the chamber temperature to below ambient.

\section{Carotenoid recovery}

"Total recovery" was determined by flow injection analysis (FIA). To perform the FIA, the HPLC column was replaced by a $2000 \mathrm{~mm} \times 0.8 \mathrm{~mm}$ I.D. PTFE tubing and the individual carotenoid solutions were introduced using a manual injector fitted with a $20-\mu l$ loop and recording the peak area at 445 $\mathrm{nm}$. This process was repeated five times for each carotenoid. Then the HPLC column was replaced and each individual carotenoid solution was injected onto the column and "peak area" was recorded. Recovery of carotenoids from the HPLC column was determined by dividing the mean "peak area" by mean "total recovery" of the five injections of each carotenoid solution.

\section{RESULTS AND DISCUSSION}

The carotenoids included in the test mixture span a wide polarity range and represent $\beta, \beta ; \beta, \varepsilon ; \psi, \psi$; monoketo; monohydroxy; and dihydroxy carotenoids. These compounds account for approximately $90 \%$ of carotenoids in human blood and the 


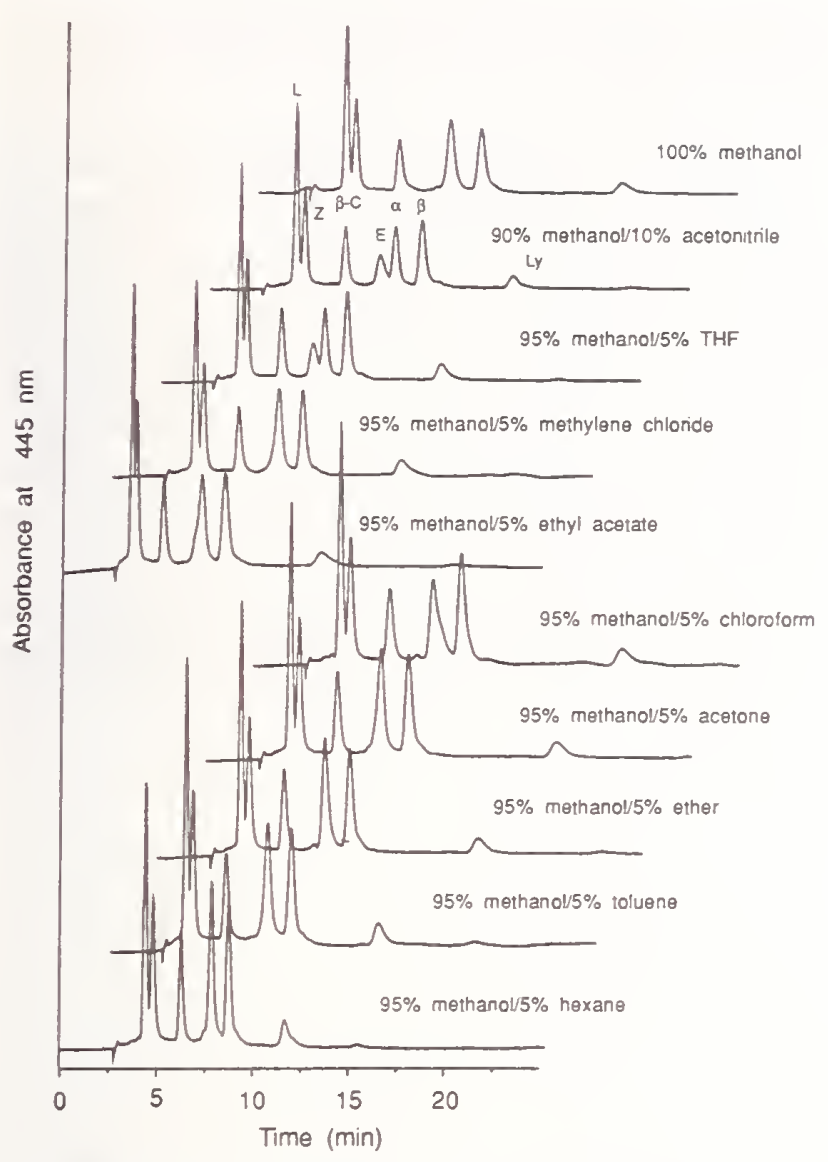

Fig. 1. The effect of nine solvent modifiers on the separation of seven carotenoids. Chromatographic conditions: Vydac 201TP $\mathrm{C}_{18}, 5 \mu \mathrm{m}, 250 \times 4.6 \mathrm{~mm}$ I.D. column; mobile phase as listed. $1.0 \mathrm{ml} / \mathrm{min}$ : UV-VIS at $445 \mathrm{~nm}$ : column temperature $25^{\circ} \mathrm{C}$. Peaks: $\mathrm{L}=$ lutein: $\mathrm{Z}=$ zeaxanthin: $\beta$-C $=\beta$-cryptoxanthin: $\mathrm{E}=$ echinenone; $x=x$-carotene; $\beta=\beta$-carotene; $\mathrm{Ly}=$ lycopene.

three major carotenoids in the US diet $[7,16]$. Therefore, an HPLC separation developed using this mix should find wide applicability to both food and serum carotenoid analyses.

A wide pore $(300 \AA)$, polymerically bonded $C_{18}$ column with "biocompatible" frits was incorporated based on results of a previous column evaluation study in which polymerically modified $\mathrm{C}_{18}$ columns demonstrated greater resolution of the carotenoids in the ethanolic mixture [13]. Also for duplicate HPLC columns with different frit materials, recovery was improved in columns fitted with "biocompatible" materials (titanium and Hastelloy C) rather than stainless steel $[13,15]$. In the same study, total carotenoid recovery was found to be higher for nearly all columns when using methanol-based

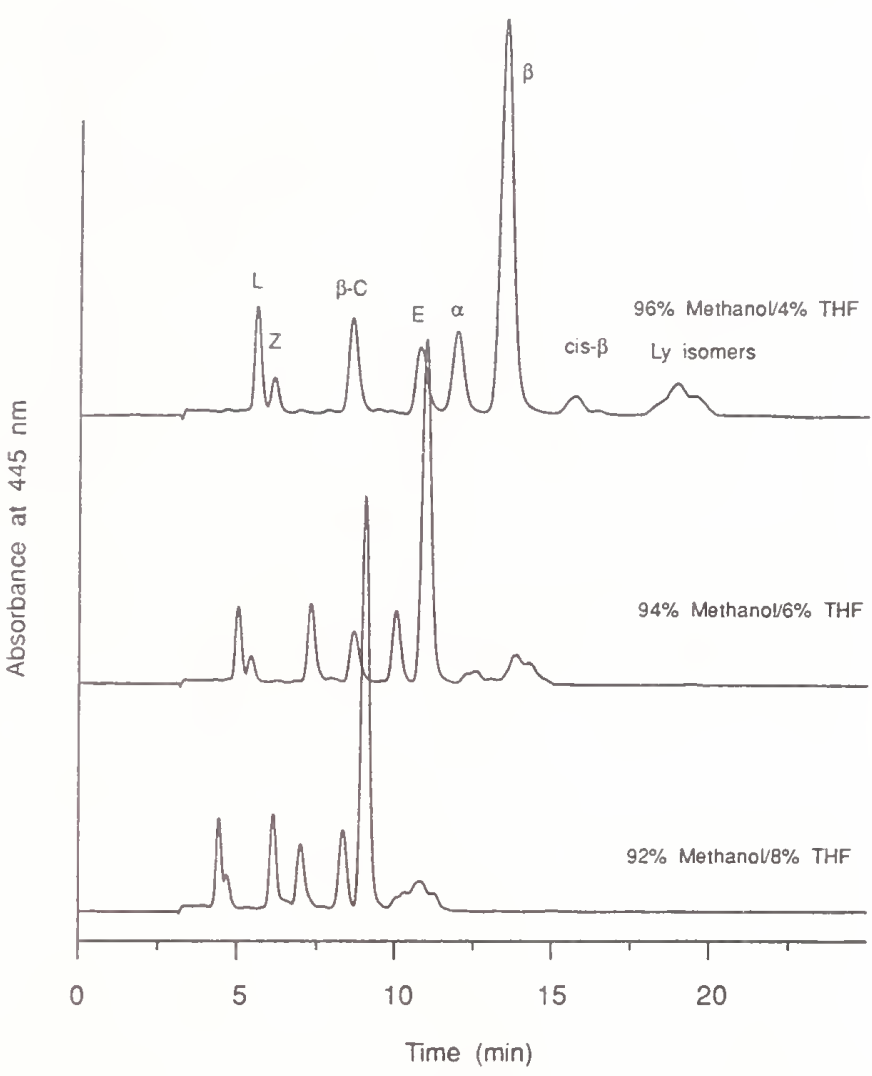

Fig. 2. The effect of three concentrations of THF on the separation of seven carotenoids. Chromatographic conditions as in Fig. 1. Peaks: cis- $\beta=\beta$-carotene cis isomers; Ly isomers = lycopene isomers; other peaks as in Fig. I.

mobile phases rather than acetonitrile-based mobile phases. In the case of the polymeric $\mathrm{C}_{18}$ columns, the use of methanol actually resulted in improved carotenoid resolution. For this reason, our initial optimization began with a methanol-based mobile phase.

The influence of the mobile phase modifiers on the separation of the seven carotenoids in the test mix is illustrated in Fig. 1. With this particular column and $100 \%$ methanol as the mobile phase, echinenone coelutes with $\alpha$-carotene, and lycopene is too far removed from $\beta$-carotene, substantially increasing analysis time. The only two modifiers that facilitated the separation of echinenone and $\alpha$-carotene were acetonitrile and THF. Both solvents also selectively reduced the retention time of lycopene. The solubility of both the xantholphylls and carotenes is greater in THF than acetonitrile [17]. Therefore to improve carotenoid solubility, THF was selected as the modifier; although the resolution of the 
lutein/zeaxanthin pair and echinenone/ $\alpha$-carotene pair was slightly decreased.

Mobile phases of methods previously developed using a similar $\mathrm{C}_{18}$ column have been modified with chloroform. THF, and a mixture of acetonitrileTHF $[11,12,18]$. Each of these reports illustrated the superior selectivity of the polymeric $\mathrm{C}_{18}$ column toward carotenoid compounds and especially geometric isomers, however, none of these reports addressed the separation of the carotenoid pairs discussed above.

The effect of varying the proportion of THF from 4 to $8 \%$ on the separation of the carotenoid mixture is illustrated in Fig. 2. As the THF concentration increases, resolution decreases between the pairs lutein/zeaxanthin and $\beta$-carotene/lycopene. At a col-

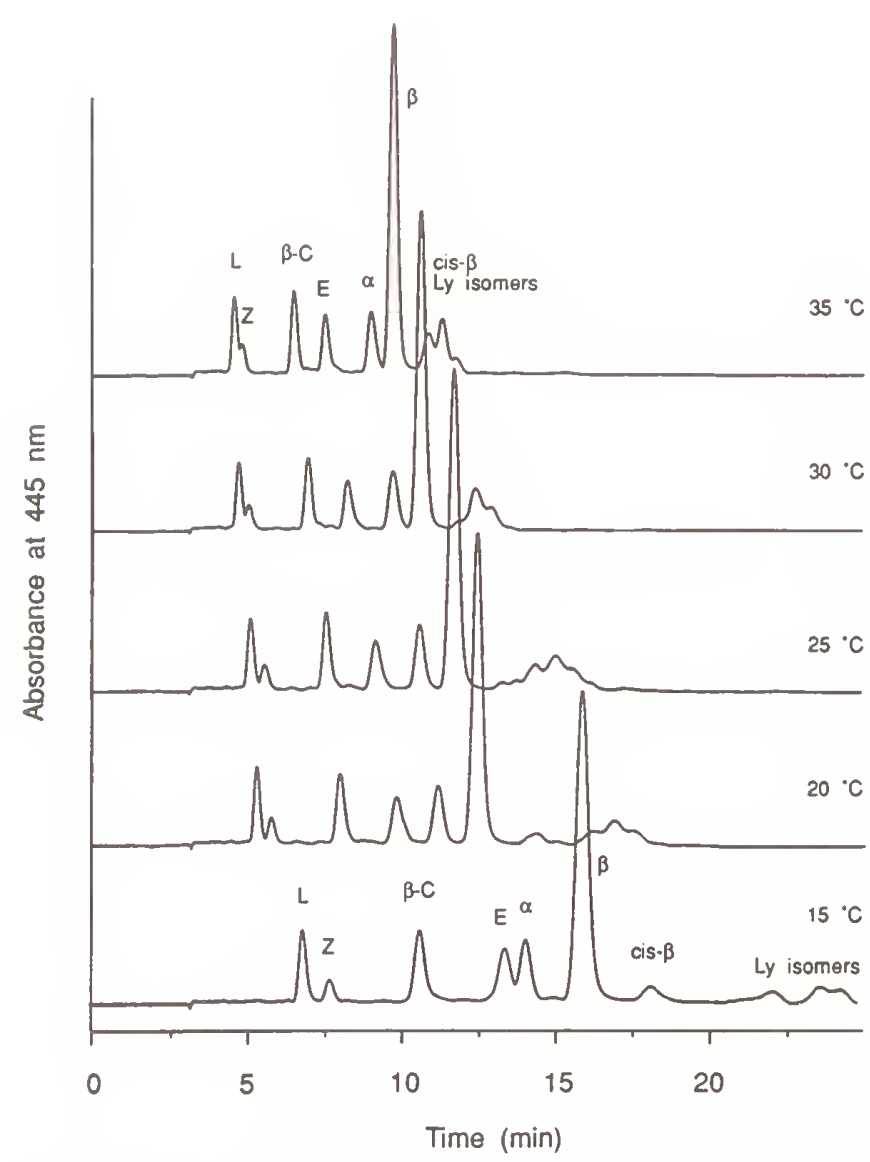

Fig. 3. The effect of five column temperatures on the separation of seven carotenoids. Chromatographic conditions: Vydac 201 TP $C_{18}$ column; $5 \%$ THF in methanol; $1.0 \mathrm{ml} / \mathrm{min}$; UV/VIS at $445 \mathrm{~nm}$; column temperature as listed. Peak identifications as in Fig. 2. umn temperature of $25^{\circ} \mathrm{C}, 3$ to $5 \%$ THF results in good separation of all the carotenoids in the mixture.

Previously, our laboratory has reported that column temperature alters column selectivity, specifically with respect to solute shape recognition $[19,20]$. Using $5 \%$ THF in methanol, column temperature was varied between 15 and $35^{\circ} \mathrm{C}$ to determine if the separation of structurally similar carotenoids could be improved (Fig. 3). At $15^{\circ} \mathrm{C}$ echinenone and lycopene are more strongly retained with respect to $\beta$-carotene $\left(\alpha_{\mathrm{E} / \beta} 0.80\right.$ vs 0.67 , and $\alpha_{1 \mathrm{y} / \beta}=$ 1.60 vs. 1.24 , respectively, at 15 and $35^{\circ} \mathrm{C}$ ). As a result, at $15^{\circ} \mathrm{C}$, echinenone and $\alpha$-carotene are not baseline resolved and lycopene elutes distant from the other carotenoids. Beginning at $25^{\circ} \mathrm{C}$ and

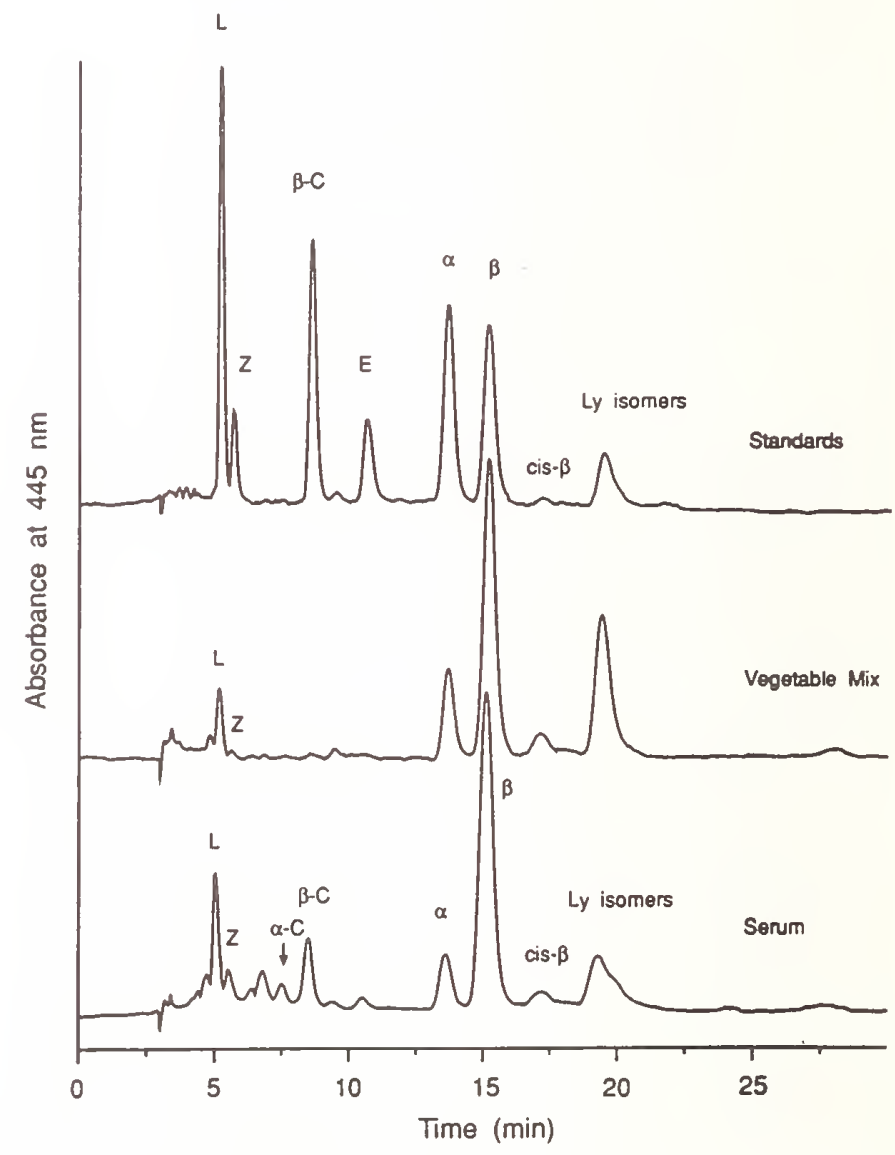

Fig. 4. The separation of serum and mixed diet carotenoids using the optimized HPLC method. Chromatographic conditions: Vydac 201TP $\mathrm{C}_{18}$ column; $5 \%$ THF in methanol; $1.0 \mathrm{ml} / \mathrm{min}$; UV/ VIS at $445 \mathrm{~nm}$. Peaks: $\mathrm{L}=$ lutein; $\mathrm{Z}=$ zeaxanthin; $\alpha-\mathrm{C}=$ $\alpha$-cryptoxanthin; $\beta$-C $=\beta$-crytoxanthin; $\alpha=\alpha$-carotene; $\beta=$ $\beta$-carotene; cis- $\beta=\beta$-carotene; cis- $\beta=\beta$-carotene cis isomers; Ly isomers $=$ lycopene isomers. 
above, resolution decreases between the lutein/ zeaxanthin pair and $\beta$-carotene/lycopene pair. A column temperature of $20^{\circ} \mathrm{C}$ was selected, because all carotenoids in the mixture are well resolved from each other, a baseline separation exists between the trans isomer of $\beta$-carotene and its geometric isomers, and there is partial resolution of the geometric isomers of lycopene. Lesellier et al. [21] recently described the separation of trans/cis- $\alpha$ - and $\beta$-carotenes and observed that temperature was a significant factor in optimizing the separation of these isomers. Using a narrow pore $(80 \AA)$ polymeric $\mathrm{C}_{18}$ column, they identified $21-22^{\circ} \mathrm{C}$ as the optimum temperature for the separation of trans- $\alpha$ - and $\beta$-carotene from their cis isomers. However, using the narrow pore column they were unable to resolve the cis isomers from each other. Previously, we have demonstrated the separation of individual geometric isomers of $\beta$-carotene using a wide pore polymeric $\mathrm{C}_{18}$ columns [22].

In our recent column evaluation study [13], we found that total carotenoid recovery from the HPLC column was influenced by the column packing, mobile phase, and frit material. The percent recovery and standard deviation of individual carotenoids measured by FIA using the optimized HPLC system described above are: lutein, $96 \pm$ $6 \%$; zeaxanthin, $107 \pm 9 \% ; \beta$-cryptoxanthin, $94 \pm$ $5 \%$; echinenone, $92 \pm 6 \% ; \alpha$-carotene, $95 \pm 3 \%$; $\beta$-carotene, $96 \pm 3 \%$; and lycopene, $108 \pm 6 \%$. Recovery of each carotenoid was greater than $92 \%$ and total carotenoid recovery was $98 \pm 6 \%$. This demonstrates that the system not only resolves these major carotenoids but also elutes them quantitatively from the LC column.

The applicability of the method is demonstrated in Fig. 4 in which the separations of serum carotenoids and dietary carotenoids are illustrated. Most isocratic reversed-phase HPLC methods fail to resolve lutein and zeaxanthin when the capacity factor $\left(k^{\prime}\right)$ for the hydrocarbon carotenoids is kept below 10 [7-9]. Previously, polymeric $C_{18}$ phases have been reported to exhibit superior ability to recognize subtle differences in molecular structure $[20,22,23]$. In this application, the lutein/zeaxanthin pair, geometric isomers of $\beta$-carotene (trans/9cis/13-cis), and geometric isomers of lycopene (trans/13-cis) are separated within $20 \mathrm{~min}$. Although superior to monomeric $\mathrm{C}_{18}$ phases in many ways, polymeric $\mathrm{C}_{18}$ columns are not without problems. The total carbon load is lower in wide-pore polymeric phases (due to the lower surface areas of wide pore silicas) which results in weaker retention of the carotenoids and limits the amount and type of modifier used in the mobile phase. The large pore diameter and thick surface coating slows mass transfer resulting in slightly broader peaks. Lastly, column reproducibility from various production lots tends to be more variable than for monomeric $\mathrm{C}_{18}$ phases [13]. Because of these difficulties, duplication of the results illustrated in this manuscript with a different polymeric $\mathrm{C}_{18}$ column may require slight modifications in the mobile phase composition, but similar separations should be attainable.

In summary, the isocratic separation of carotenoids was optimized using a polymeric $\mathrm{C}_{18}$ column. Stainless steel frits were replaced with "biocompatible" frits to minimize oxidative degradation of the analytes and a methanol-based mobile phase was used to improve selectivity and recovery. Nine solvent modifiers were investigated to determine their effect on separation selectivity and THF was found to be the most beneficial modifier. Various combinations of THF composition and column temperature were assessed to achieve the best overall separation. The recovery of individual carotenoids and a mixture of seven carotenoids from the HPLC column was nearly $100 \%$.

\section{ACKNOWLEDGEMENTS}

The authors gratefully acknowledge the helpful discussions with Lane C. Sander regarding stationary phase chemistry and the assistance of Katherine S. Epler with the initial column evaluations. The authors are grateful to Chris Nelson of Kemin Industries and Hemmige Bhagavan of Hoffmann-La Roche for their generous contributions of carotenoids.

This work is taken in part from a dissertation submitted by N.E.C. to the Graduate School of the University of Maryland in partial fulfillment of the requirements for the Ph.D. degree in Nutritional Sciences.

Certain commercial equipment, instruments, or materials are identified in this paper to specify adequately the experimental procedure. Such identification does not imply recommendation of endorse- 
ment by the National Institute of Standards and Technology, nor does it imply that the materials or equipment identified are neccesarily the best available for the purpose.

\section{REFERENCES}

1 M. S. Menkes, G. W. Comstock, J. P. Vuilleumeir, K. J. Helsing, A. R. Rider and R. Brookmeyer, N. Engl. J. Med., 20 (1986) 1250.

2 G. A. Colditz, L. G. Branch, R. J. Lipnick, W. C. Willett, B. Rosner, B. M. Posner and C. H. Hennekens, Am. J. Clin. Nutr., 41 (1985) 32.

3 W. C. Willett, B. F. Polk, B. A. Underwood, M. J. Stampfer, S. Pressel, B. Rosner, J. O. Taylor, K. Schneider and C. G. Hames, N. Engl. J. Med., 310 (1984) 430.

4 R. Peto, R. Doll, J. D. Buckley and M. B. Sporn, Nature (London), 290 (1981) 201.

5 G. W. Burton and K. U. 1ngold, Science (Washington, D.C.). 224 (1984) 569.

6 J. M. Gaziano, J. E. Manson, P. M. Ridker, J. E. Buring and C. H. Hennekens, Circulation, 82 (1990) 796.

7 J. G. Bieri, E. D. Brown and J. C. Smith, J. Liq. Chromatogr., 8 (1985) 473.

8 C. S. Yang and M.-J. Lee, J. Nutr. Growth Cancer, 4 (1987) 19.
9 A. L. Sowell, D. L. Huff, E. W. Gunter and W. J. Driskell, J. Chromatogr., 431 (1988) 424.

10 D. A. Kalman, G. E. Goodman, G. S. Omenn, G. Bellamy and B. Rollins, J. Natl. Cancer Inst., 79 (1987) 975.

11 R. J. Bushway, J. Liq. Chromatogr., 8 (1985) 1527.

12 F. W. Quackenbush, J. Liq. Chromatogr., 10 (1987) 643.

13 K. S. Epler, L. C. Sander, S. A. Wise, R. G. Ziegler and N. E. Craft, J. Chromatogr., 595 (1992) in press.

14 L. C. Sander and S. A. Wise, LC. GC. 8 (1990) 378.

15 D. W. Nierenberg and D. C. Lester, J. Nutr. Growth Cancer, 3 (1986) 215.

16 N. E. Craft, S. A. Wise, and J. H. Soares, Jr., J. Agric. Food Chem., submitted for publication.

17 N. E. Craft and J. H. Soares, Jr., J. Agric. Food Chem., in press.

18 F. W. Quackenbush and R. L. Smallidge, J. Assoc: Off. Anal. Chem., 69 (1986) 767.

19 L. C. Sander and N. E. Craft. Anal. Chem., 62 (1990) 1545.

20 L. C. Sander and S. A. Wise, Anul. Chem., 6l (1989) 1749.

21 E. Lesellier, C. Marty, C. Berset and A. Tchapla, J. High Resolut. Chromatogr. Chromatogr. Commun., 12 (1989) 447.

22 N. E. Craft, L. C. Sander and H. F. Pierson. J. Micronutr. Anal., 8 (1991) 209.

23 L. C. Sander and S. A. Wise, Anal. Chem., 56 (1984) 504. 


\title{
Development of Engineered Stationary Phases for the Separation of Carotenoid Isomers
}

\author{
Lane C. Sander, ${ }^{`}$ Katherine Epler Sharpless, Neal E. Craft, ${ }^{\dagger}$ and Stephen A. Wise \\ Organic Analytical Research Division, Chemical Science and Technology Laboratory, National Institute of \\ Standards and Technology, Gaithersburg, Maryland 20899
}

A variety of bonded phase parameters (endcapping, phase chemistry, ligand length, and substrate parameters) were studied for their effect on column retention and selectivity toward carotenoids. Decisions were made on how each of these variables should be optimized based on the separation of carotenoid and polycyclic aromatic hydrocarbon test probes. A column was designed with the following properties: high absolute retention, enhanced shape recognition of structured solutes, and moderate silanol activity. These qualities were achieved by triacontyl $\left(C_{30}\right)$ poly meric surface modification of a moderate pore size $(\sim 20 \mathrm{~nm})$, moderate surface area $(\sim 200$ $\mathrm{m}^{2} / \mathrm{g}$ ) silica, without subsequent endcapping. The effectiveness of this "carotenoid phase" was demonstrated for the separation of a mixture of structurally similar carotenoid standards, an extract of a food matrix Standard Reference Material, and a $\beta$-carotene dietary supplement under consideration as an agent for cancer intervention/prevention.

Carotenoids are highly conjugated polyprenoids found in a variety of natural sources including fruits and vegetables. Interest in the separation, identification, and quantification of these compounds is largely motivated by perceived health benefits; however, carotenoids are also widely utilized as natural pigmenting agents. Carotenoids constitute a class of molecules with diverse properties. Carotenoid hydrocarbons (carotenes) are nonpolar, whereas oxy-substituted carotenoids (referred to as xanthophylls) are polar. Subtle molecular differences exist within these groups, and geometric and positional isomers of carotenoids are common. Because of the complexity of carotenoid extracts and the minor shape differences among carotenoid isomers, separation of individual species is difficult.

In reversed-phase liquid chromatography (LC) systems, the retention of nonpolar compounds is often attributed to solvophobic interactions, but other properties of the solute may provide an additional basis for separation. For example, with polycyclic aromatic hydrocarbons (PAHs), separations of isomers can be achieved on the basis of molecular shape. ${ }^{1-9}$ For solutes with oxygen-containing substituents, dipole or

${ }^{\dagger}$ Present address: Southern Testing \& Research Labs, Inc., Wilson, NC 27893. (1) Sander, L. C.; Wise, S. A. J. Chromatogr. 1993, 656, 335-351.

(2) Sander, L. C.; Wise, S. A. LC GC 1990, 8, 378-390.

(3) Wise, S. A.; Sander, L. C.; Lapouyade, R.; Garrigues, P. J. Chromarogr. 1990, $514,111-122$.

(4) Wise, S. A.; Sander, L. C.; Chang, H.; Markides, K. E.; Lee, M. L. Chromasographia 1988, 25, 473-480.

(5) Sander, L. C.; Wise, S. A. Advances in Chromatography, Giddings, J. C., Grushka, E., Cazes, J., Brown, P. R., Eds.; Marcel Dekker: New York, 1986; Vol. 25, pp 139-218.

(6) Wise, S. A.;Sander, L. C. HRC CC. J. High Resolut. Chromatogr. Chromatogr. Commun. 1985, 8, 248-255.

(7) Cole, S. R.; Dorsey, J. G. J. Chromarogr. 1993, 635, 177-186.

(8) Sentell, K. B.; Dorsey, J. G. J. Chromatogr. 1989, 46I, 193-207. hydrogen bonding interactions may provide another basis for separation. Stationary phases can be designed with properties engineered for the solution of a given separation problem by understanding the cause and effect relationship between bonded phase properties and solute interactions.

The separation of carotene and xanthophyll mixtures has been the subject of considerable research effort spanning several decades. ${ }^{10-26}$ Most LC separations of carotenes and xanthophylls have been developed using reversed-phase systems, ${ }^{27-31}$ although normal-phase separations (particularly of geometric isomers of $\beta$-carotene) have also been reported. ${ }^{32-36}$ In a study of the retention behavior of carotenoid compounds with over 60 commercial $C_{18}$ columns, better separations of nonpolar carotenoids were usually possible with columns prepared using polymeric surface modification chemistry compared with monomeric surface chemistry. ${ }^{37}$ Variations in the retention behavior of polar carotenoids (i.e., xanthophylls) were observed among both monomeric and polymeric $\mathrm{C}_{18}$ phases.

(9) Sentell, K. B.; Dorsey, J. G. Anal. Chem. 1989, 61, 930-934.

(10) Zechmeister. L. Chem. Rev. 1944, 34, 267-344.

(11) Bickoff, E. M. Anal. Chem. 1948, 20, 51-54.

(12) Williams, R. C.; Schmit, J. A.; Henry, R. A. J. Chromatogr. Sci. 1972, 10, 494-501.

(13) Weedon, B. C. L. Pure Appl. Chem. 1979, 5I, 435-445.

(14) Schwartz, S. J.; von Elbe, J. H. J. Liq. Chromatogr. 1982, 5 (suppl. I), 43-73.

(I5) Krinsky, N. I.; Welankiwar, S. Methods Enzymol. 1984, 105, 155-162.

(16) Bushway, R. J. J. Liq. Chromatogr. 1985, 8, 1527-1547.

(17) Ruddat, M.; Will, O. H., III Methods Enzymol. 1985, 111, 189-200.

(18) Ruedi, P. Pure Appl. Chem. 1985, 57, 793-800.

(19) Schwartz, S. J.; Patroni-Killam, M. J. Agric. Food Chem. 1985, 33, 1160 1163.

(20) Taylor, R. F. Advances in Chromatography. Volume 22; Giddings, J. C., Grushka, E.; Cazes, J., Brown, P. R., Eds.; Marcel Dekker: New York, 1985; PP 157-213.

(21) Chandler, L. A.; Schwartz, S. J. J. Food Sci. 1987, 52, 669-672.

(22) Quackenbush, F. W. J. Lig. Chromatogr. 1987, 10, 643-653.

(23) Craft, N.; Sander, L. C.; Pierson, H. F. J. Micronutr. Anal. 1990, 8, 209-221.

(24) Matus, Z;; Ohmacht, R. Chromatographia 1990, 30, 318-322.

(25) Granado, F.; Olmedilla, B.; Blanco, I.; Rojas-Hidalgo, E. J. Liq. Chromatogr. 1991, 14, 2457-2475.

(26) Tsukida, K. Methods Enzymol. 1992, 213, 291-298.

(27) Braumann, T.; Grimme, L. H. Blochim. Blophys. Acta 1981, 637, 8-17.

(28) Nelis, H. J. C. F.; De Leenheer, A. P. Anal. Chem. 1983, 55, 270-275.

(29) Will, O. H., Ill; Ruddat, M. LC Mag. 1985, 2, 610-612.

(30) Lesellier, E.; Marty, C.; Berset, C.; Tchapla, A. J. High Resolut. Chromatogr. $1989,12,447-454$

(31) Craft, N. E. Methods Enzymol. 1992, 213, 185-205.

(32) Berg, W. F.; Sandler, L. Nature 1935, 136, 914-915.

(33) Nelis, H. J. C. F.; van Steenberge, M. M. Z.; Lefevere, M. F.; De Leenheer, A. P. J. Chromalogr. 1986, 353, 295-302.

(34) Hashimoto, H.; Koyama, Y. J. Chromatogr. 1988, 448, 182-187.

(35) Koyama, Y.; Hosomi, M.; Miyata, A.; Hashimoto, H.; Reames, S. A. J. Chromalogr. 1988, 439, 417-422.

(36) Rhodes, S. H.; Netting, A. G.; Milborrow, B. V. J. Chromatogr. 1988, 442 412-419.

(37) Epler, K. S.; Sander, L. C.; Ziegler, R. G.; Wise, S. A.; Craft, N. E. J. Chromatogr. 1992, 595, 89-101. 
In this study the effect of various bonded phase parameters on retention is examined for polar and nonpolar carotenoids. Through the control of appropriate bonded phase variables, the development of a stationary phase tailored for the separation of carotenoids is described. The utility of this column is demonstrated for the separation of standards as well as complex food extracts and dietary supplements.

\section{EXPERIMENTAL SECTION}

Materials and Reagents. Carotene and xanthophyll standards were obtained from a variety of sources. trans- $\alpha$ - and trans- $\beta$-carotene, and trans-lycopene were obtained from Sigma Chemical Co. (St. Louis, MO), echinenone, zeaxanthin, astaxanthin, $\delta$-carotene, capsanthin, canthaxanthin, and 9-cis-, 13-cis-, and 15-cis- $\beta$-carotene were obtained from HoffmanLaRoche (Nutley, NJ, and Basel, Switzerland), $\beta$-cryptoxanthin was obtained from Atomergic Chemetals (Farmingdale, NY), and lutein was obtained from Kemin Industries (Des Moines, IA). A $\beta$-carotene preparation containing a high percentage of cis isomers was obtained from Betatene Ltd. (Melbourne, Australia). Octadecyltrichlorosilane, dimethyloctadecylchlorosilane, and triacontyltrichlorosilane were obtained from Hūls America, Inc. (Piscataway, NJ). Four microparticulate silicas were used in the preparation of bonded phases: YMC SIL200 5- $\mu \mathrm{m}$ silica (YMC Inc., Wilmington, NC), IMPAQ $2005-\mu \mathrm{m}$ silica (PQ Corporation, Conshohocken, PA), Kromasil $2005-\mu \mathrm{m}$ silica (Eka Nobel, Bohus, Sweden), and Vydac TP 5- $\mu \mathrm{m}$ silica (Separations Group, Hesperia, CA). For comparisons of separations made with commercial columns, a Zorbax (monomeric $\mathrm{C}_{18}$ ) column (Mac Mod Analytical, Inc., Chadds Ford, PA) and a Hypersil Green PAH (polymeric $C_{18}$ ) column (Shandon HPLC, Cheshire, UK) were employed. HPLC-grade solvents were used in chromatographic separations. Candidate Standard Reference Material (SRM) 2383, Vitamins and Carotenoids in Food, and SRM 869, Column Selectivity Test Mixture for Liquid Chromatography, were obtained from the NIST Standard Reference Materials Program (NIST, Gaithersburg, MD).

Bonded Phase Synthesis. Monomeric and polymeric $\mathrm{C}_{18}$ phases were prepared using a previously published procedure. ${ }^{38.39}$ Polymeric $\mathrm{C}_{30}$ phases were prepared in a similar fashion, as follows. Approximately $10 \mathrm{~g}$ of triacontyltrichlorosilane was added to $100 \mathrm{~mL}$ of xylene and heated to boiling. After dissolution, the mixture was filtered while hot to remove solid impurities. In a separate container $\sim 4 \mathrm{~g}$ of 20 -nm pore size, $5-\mu \mathrm{m}$ particle size silica was dispersed in $25 \mathrm{~mL}$ of $x y l e n e$. This silica slurry was added to the hot silane reaction mixture. One milliliter of water was added to initiate the polymerization reaction. The slurry was again heated and refluxed for 3-6 h. The bonded silica was filtered (hot) using a sintered glass frit funnel and washed with xylene, acetone, methanol, water, acetone, and pentane. Samples for carbon analysis were dried at $\sim 100^{\circ} \mathrm{C}$ under reduced pressure for $4 \mathrm{~h}$, and shipped to Galbraith Laboratories (Knoxville, TN).

Chromatography. Separations of carotenoid standards and extracts were carried out using a linear mobile phase gradient

(38) Sander, L. C.; Wise, S. A. Anal. Chem. 1984, 56, 504-510.

(39) Sander, L. C.: Wise, S. A. Polynuclear Aromatic Hydrocarbons: Eighth International Symposium on Mechanism. Method and Metabolism, Cooke. M. W., Dennis, A. J., Eds.; Battelle Press: Columbus, OH. 1983; pp 11331144 . from $81: 15: 4$ to $6: 90: 4$ methanol/methyl-tert-butyl ether (MTBE)/water, over $90 \mathrm{~min}$, at a flow rate of $1 \mathrm{~mL} / \mathrm{min}$. Columns were thermostated at $20^{\circ} \mathrm{C}$ with a water jacket. Detection for all separations was at $450 \mathrm{~nm}$. Isocratic separations of the high $c i s-\beta$-carotene mixture were carried out at $3^{\circ} \mathrm{C}$ using an 80:20 acetonitrile/MTBE mobile phase (90:10 acetonitrile/MTBE for commercial monomeric and polymeric $\mathrm{C}_{18}$ phases).

Extraction of SRM 2383. SRM 2383 Vitamins and Carotenoids in Food was extracted and saponified in preparation for carotenoid analysis using the modified procedure of Scott. ${ }^{40}$ Approximately $2.0 \mathrm{~g}$ of the food mixture was combined with $0.2 \mathrm{~g}$ of calcium carbonate, $5 \mathrm{~mL}$ of water, and $100 \mathrm{~mL}$ of $50 / 50 \mathrm{THF} /$ methanol, and the mixture was homogenized for $1 \mathrm{~min}$. This mixture was vacuum-filtered, and the filter cake was rinsed with $50-\mathrm{mL}$ portions of $50 / 50$ $\mathrm{THF} /$ methanol uritil the orange color was removed from the solids. The filtrate was transferred to a separatory funnel, 50 $\mathrm{mL}$ of a $10 \%(\mathrm{w} / \mathrm{v})$ sodium chloride solution was added, and the analytes were extracted 3 times with $50-\mathrm{mL}$ portions of diethyl ether. The ether was evaporated to a volume of approximately $50 \mathrm{~mL}$. This solution was shaken twice with petroleum ether to reextract the analytes, and the petroleum ether phase was eva porated to approximately $500 \mu \mathrm{L}$. Ethanol was added to the solution to yield a total volume of $5 \mathrm{~mL}$.

These extracts were further processed to convert xanthophyll esters to free xanthophylls using the following saponification procedure: A 3-mL aliquot of the food extract was combined with $0.1 \mathrm{~mL}$ of $20 \%(\mathrm{w} / \mathrm{v})$ pyrogallol $(1,2,3-$ benzenetriol) in methanol, $1.5 \mathrm{~mL}$ of $40 \%$ potassium hydroxide in methanol, and $1.5 \mathrm{~mL}$ of methanol. This mixture was allowed to sit at room temperature for $30 \mathrm{~min}$ and was then extracted with three 10 -mL portions of 50/50 petroleum ether/ diethyl ether. The combined extracts were evaporated under a stream of nitrogen to a viscous solution. Water and petroleum ether were added to extract the analytes, and the petroleum ether phase was removed and evaporated. The resultant waxy substance was redissolved in a 50/50 mixture of ethyl acetate/ethanol to total $3 \mathrm{~mL}$.

\section{RESULTS AND DISCUSSION}

To design a stationary phase with properties tailored for the separation of carotenoid compounds, the effect of various sorbent parameters on retention was examined using representative carotenoid probes and other test mixtures. The influence that bonded phase chemistry exhibits on PAHs has been studied in considerable detail.4-6,38,41-43 In general, columns prepared using polymeric surface modification chemistry exhibit enhanced shape selectivity toward isomers and other groups of compounds with similar molecular structure. This is in comparison to columns prepared using monomeric surface modification chemistry, which generally exhibit greater absolute retention, but reduced shape recognition. A trend toward enhanced shape recognition has also been observed for increasing phase length, for both monomeric and polymericalkyl bonded phases. ${ }^{44}$ Although silanol activity

(40) Scott K. J. Institute of Food Research, Norwich, UK. Personal communication, 1993.

(41) Sander, L. C.; Parris, R. M.; Wise, S. A.; Garrigues, P. Anal. Chem. 1991, 63, 2589-2597.

(42) Sander, L. C.; Wise, S. A. Anal. Chem. 1989. 61, 1749-1754.

(43) Sander, L. C.; Wise, S. A. J. Chromatogr. 1984, 316, 163-181. 


\section{Carotenoids}

Xanthophylls

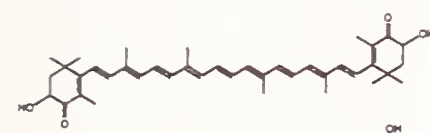

Astaxanthin

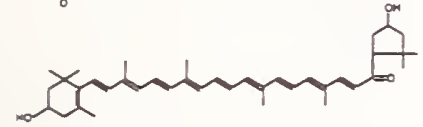

Capsanthin

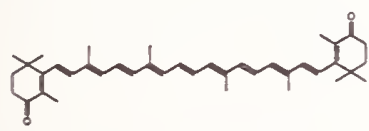

Canthaxanthin

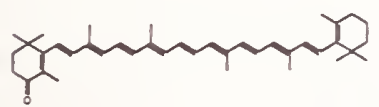

Echinenone

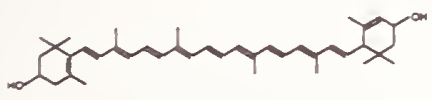

Lutein

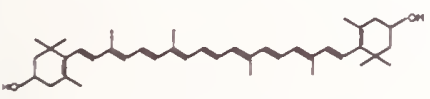

Zeaxanthin

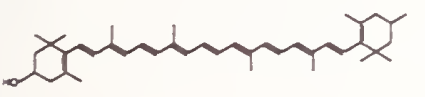

ß-Cryptoxanthin

\section{Hydrocarbons}
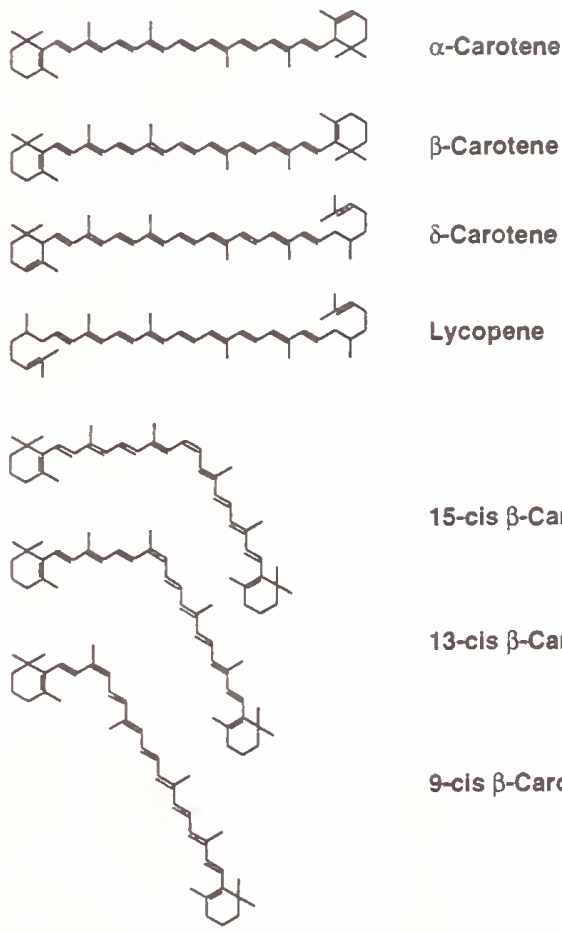

15-cis $\beta$-Carotene

13-cis $\beta$-Carotene

9-cis $\beta$-Carotene

Flgur 1. Structures of polar and nonpolar carotenoids.

has been shown to have no effect on shape selectivity for nonpolar compounds such as PAHs, ${ }^{1}$ certain carotenoid compounds have oxygen-containing substitutents and exhibit considerable polarity. For this reason, silanol activity has the potential for influencing carotenoid separations. Finally, substrate properties such as pore diameter and surface area have been shown to affect the mechanism of synthesis of various alkyl phases and, consequently, the overall retention behavior of the resulting sorbents. ${ }^{43}$

The structures of some of the more important common carotenoids are shown in Figure 1. Differences in the structures of these compounds are often minor. For example, $\alpha$ - and $\beta$-carotene differ in the position of a double bond in one of the terminal rings. Lutein and zeaxanthin are similarly related and can be thought of as dihydroxy analogs of $\alpha$ - and $\beta$-carotene. Cis isomers of $\beta$-carotene differ in the location of cis double bonds along the conjugated chain. $\delta$-Carotene and lycopene have one and/or both end rings opened, respectively. Xanthophylls differ in the extent and position of substitution for hydrozylation or ketonation. It is believed that geometric isomers exist for many of these compounds (e.g., cis isomers of $\alpha$-carotene), and only a small fraction of the known carotenoids are illustrated in Figure 1.

The primary goal of this work was to develop a column that exhibited significantly enhanced selectivity toward both polar and nonpolar carotenoids compared to existing commercial columns. In addition, a column with high absolute

(44) Sander, L. C.; Wise, S. A. Anal. Chem. 1987, 59, 2309-2313. retention was desired to reduce the amount of water required in the mobile phase and to provide adequate retention for polar carotenoids. The use of mobile phases containing little or no water benefit preparative carotenoid separations since solvent elimination is greatly facilitated by the absence of water. The low viscosity of such mobile phases also permits the utilization of longer columns and smaller particle diameter substrates. A column can be produced with properties optimized for specific carotenoid separations by understanding how each parameter affects carotenoid retention.

Stationary Phase Chemistry. Three octadecyl phases were prepared by different approaches. A monomeric $C_{18}$ phase was prepared on IMPAQ silica using an excess of dimethyloctadecylchlorosilane in the presence of a basic catalyst (2 $\mathrm{mL}$ of $N, N$-diethyl-m-toluamide). Another $\mathrm{C}_{18}$ phase was prepared by reaction of octadecyltrichlorosilane with the silica, under anhydrous conditions. A third $\mathrm{C}_{18}$ phase was synthesized also using octadecyltrichlorosilane, but with the addition of $1.0 \mathrm{~mL}$ of water to initiate polymerization. Carbon loading and surface coverage values for the stationary phases are listed in Table 1.

Separations utilizing these columns are shown in Figure 2. The chromatograms at the left of the figure are for SRM 869. Column Selectivity Test Mixture for Liquid Chromatography. The use of this material to characterize stationary phase type (monomeric vs. polymeric) and shape recognition properties has been detailed in previous publications, $5,38,45-47$ The elution order of the three PAH probes (benzo $[a]$ pyrene $(\mathrm{BaP})$, phenanthro[3,4-c]phenanthrene ( $\mathrm{PhPh})$, and 1,2:3,4: 
Table 1. Sonded Phase Propertios stationary phase type

monomeric monofunctional $\mathrm{C}_{18}$ monomeric trifunctional $\mathrm{C}_{18}$ (anhydrous synthesis)

polymeric $\mathrm{C}_{18}$

polymeric $\mathrm{C}_{18}$ (not endcapped)

polymeric $\mathrm{C}_{18}$ (TMCS endcapped)

polymeric $\mathrm{C}_{18}$ (HMDS endcapped)

polymeric $\mathrm{C}_{30}$

polymeric $\mathrm{C}_{30}$

polymeric $\mathrm{C}_{30}$

$\begin{array}{lc}\text { silica } & \text { surface area }\left(\mathrm{m}^{2} / \mathrm{g}\right) \\ \text { IMPAQ 200 } & 240 \\ \text { IMPAQ 200 } & 240 \\ \text { IMPAQ 200 } & 240 \\ \text { Vydac TP } & \sim 75^{b} \\ \text { Vydac TP } & \sim 75^{b} \\ \text { Vydac TP } & \sim 75^{b} \\ \text { IMPAQ 200 } & 240 \\ \text { YMC SIL200 } & 200^{b} \\ \text { Kromasil 200 } & 196\end{array}$

percent carbon

coverage $\left(\mu \mathrm{mol} / \mathrm{m}^{2}\right)$

12.79

3.07

3.03

20.01

7.99

5.44

7.84

8.33

23.34

19.41

14.46

${ }^{a}$ Measured values. ${ }^{b}$ Nominal values reported by the manufacturer.

\section{A}
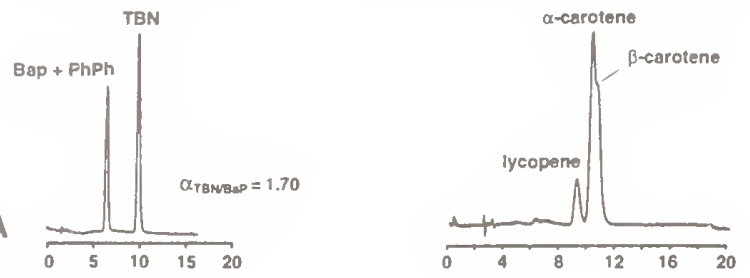

B

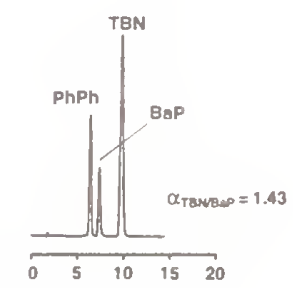

c
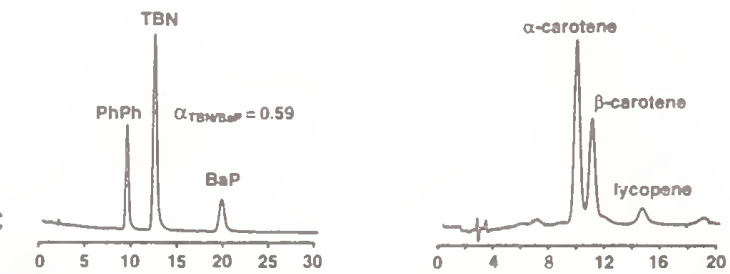

Flgure 2. Column selectivity comparison for various $C_{18}$ phases prepared using monomeric and polymeric syntheses: (A) monomeric, monofunctionai synthesis; (B) monomeric, trifunctional synthests (anhydrous); (C) polymeric synthesis. Separation conditions were as follows: left (SRM 869), $2 \mathrm{~mL} / \mathrm{min}, 85: 15$ acetonitrile/water; right (carotenes), $1.5 \mathrm{~mL} / \mathrm{min}, 80: 20$ methanol/ethyl acetate.

5,6:7,8-tetrabenzonaphthalene (TBN)) is indicative of column type. Furthermore, the selectivity factor $\alpha_{\mathrm{TBN} / \mathrm{BaP}}$ provides a measure of shape selectivity for structurally similar compounds; low values of $\alpha_{\mathrm{TBN} / \mathrm{BaP}}$ indicate a high degree of shape recognition. The chroma tograms in Figure 2 for SRM 869 are typical of those observed for monomeric and polymeric $\mathrm{C}_{18}$ phases. The monomeric $\mathrm{C}_{18}$ phase prepared using the

(45) Sander, L.C.; Wise, S. A.SRM 869 Certificate of Analysis, Standard Reference Materials Program, NIST, Gaithersburg, MD, 1990.

(46) Sander, L. C.; Wise, S. A. HRCCC, J.High Resolut. Chromatogr. Chromatogr. Commun. 1988, 11, 383-387.

(47) Sander, L. C. J. Chromalogr. Sci. 1988, 26, 380-387. anhydrous trifunctional synthesis (Figure $2 \mathrm{~B}$ ) exhibits an elution order $\mathrm{PhPh}<\mathrm{BaP}<\mathrm{TBN}\left(1<\alpha_{\mathrm{TBN} / \mathrm{BaP}}<1.7\right)$ and this may indicate slight polymeric character, although it is interesting to note that the carbon loading for this stationary phase is slightly less than for the monofunctional monomeric phase.

Of more direct interest is the separation of three representative nonpolar carotenoids (namely lycopene and $\alpha$-and $\beta$-carotene). These carotenes were selected as probes based on their importance in clinical measurements, and their widespread occurrence in fruits and vegetables. The separation of these common carotenes is a prerequisite for any column designed for carotenoid measurements. With the monofunctional monomeric $\mathrm{C}_{18}$ phase (Figure $2 \mathrm{~A}$ ), lycopene was resolved from $\alpha$-and $\beta$-carotene, but $\alpha$ - and $\beta$-carotene were unresolved. Poorer resolution was observed using the trifunctional monomeric $\mathrm{C}_{18}$ phase (Figure 2B). Nearly baseline separation of the three carotenoids was achieved with the polymeric $\mathrm{C}_{18}$ phase (Figure 2C). Thus, at least for the probe compounds (nonpolar carotenes), better separations were possible using the polymeric surface modification a pproach. Several methods for the analysis of carotenoids have been reported in which polymeric $\mathrm{C}_{18}$ columns are utilized. The distinction between monomeric and polymeric $\mathrm{C}_{18}$ phases is not commonly expressed. Instead, the methods are described with a specific column brand rather than by bonded phase chemistry or retention behavior. ${ }^{16,48}$ A similar situation has existed for the separation of PAHs; however, the realization of the importance of stationary phase chemistry toward selectivity is becoming more widespread. For example, Lesellier and co-workers recently studied carotenoid retention mechanisms on commercial monomeric and polymeric $\mathrm{C}_{18}$ columns. ${ }^{49}$

Endcapping. The actual effect that silanol groups contribute toward retention and selectivity remains controversial even though silanol activity has been the subject of numerous studies.24,50-54 It is clear, however, that the extent and magnitude of any silanol effects are dependent on the nature of the solute molecules under study. Silanols can be expected to influence the retention behavior of polar solutes to a greater extent than nonpolar hydrocarbons. Traditionally, silanol

(48) Quackenbush, F. W.; Smallidge, R. L. J. Assoc. Off. Anal. Chem. 1986, 69, 767-771.

(49) Lesellier, E.; Tchapla, A.; Krstulovic, A. M. J. Chromaiogr. 1993, 645. 29-39.

(50) Nahum, A.; Horvath, C. J. Chromatogr. 1981, 203, 53-63.

(5I) Miller, M. L.; Linton, R. W.; Maciel, G. E.; Hawkins, B. L. J. Chromatogr. 1985, 319, 9-21.

(52) Jennings, E. C.; Brownlee, R. G. Anal. Chem. 1986, 58, 2895.

(53) Mant, C. T.; Hodges, R. S. Chromalographia 1987, 24, 805-814.

(54) Nawrocki, J. Chromatographia 1988, 25, 404-408. 
activity is usually considered undesirable, and secondary silanization reactions using methylsilanes (endcapping) are carried out to reduce this activity. In previous work, we investigated the influence of silanol activity and endcapping on retention behavior for a series of polycyclic aromatic hydrocarbon isomers. ${ }^{1,38} \mathrm{~A}$ single batch of a polymeric $\mathrm{C}_{18}$ phase was divided into three portions, of which two where endcapped with trimethylchlorosilane (TMCS) and hexamethyldisilazane (HMDS). The third portion was not endcapped. No difference was apparent for PAH separations carried out on the three columns, yet a fourth $C_{18}$ phase prepared by monomeric surface modification on the same silica substrate exhibited large differences in retention and selectivity. In general, column selectivity toward nonpolar solutes such as PAHs is not affected by silanol activity, but is strongly affected by column type (i.e., monomeric vs polymeric syntheses). ${ }^{1}$

Because carotenoids span a wide range of polarity and may potentially have interactions with silanols, the effect of endcapping on retention behavior was studied for polar and nonpolar carotenoid compounds. Endcapped and nonendcapped phases were prepared (as described above) using a single batch of a polymeric $C_{18}$ phase prepared on Vydac TP silica. Properties of the stationary phases are listed in Table 1. A slight decrease in carbon loading was measured after endcapping with TMCS, whereas endcapping with HMDS resulted in an increase in carbon loading. This phenomenon (for endcapping processes utilizing TMCS) was first reported by Berendsen et al. ${ }^{55}$ It is thought that $\mathrm{HCl}$ formed as a byproduct of endcapping acts to catalyze cleavage of a fraction of the $\mathrm{C}_{18}$ ligands, resulting in a net loss of carbon.

To examine the influence of silanol activity on carotenoid selectivity, two representative mixtures were used: $\alpha$ - and $\beta$-carotene and lycopene (nonpolar probes) and lutein and zeaxanthin (polar probes). Separations of these compounds are shown in Figure 3 for the endcapped and nonendcapped stationary phases. Also shown are separations of SRM 869. No appreciable difference exists in the retention behavior of the three columns toward the PAH probes (SRM 869). This is to be expected, since SRM 869 indicates differences in shape selectivity based on bonded phase chemistry unrelated to polarity. Separations of the three nonpolar carotenoids are also affected little by endcapping for similar reasons. In contrast, the separation of polar carotenoids lutein and zeaxanthin is influenced by silanol activity, as indicated in Figure 3. Better separation was observed with the unendcapped stationary phase (Figure 3A) compared to either of the endcapped phases (Figure 3B,C). Because the separation of lutein and zeaxanthin is of particular importance in the food science and clinical disciplines, it seems reasonable to use a synthesis approach that facilita tes this separation. The separation of nonpolar carotenoids, as indicated by the three nonpolar probes, is unaffected by the presence or absence of endcapping; therefore, the best synthesis approach would be to not endcap. Lack of endcapping further eliminates bonded phase stability problems that have recently been attributed to endcapped phases. ${ }^{56}$ These conclusions are similar to those of Matus and Ohmacht, who first reported a detailed

(55) Berendsen, G. E.; Pikaart, K. A.: de Galan, L. J. Liq. Chromatogr. 1980, 3, 1437-1464.
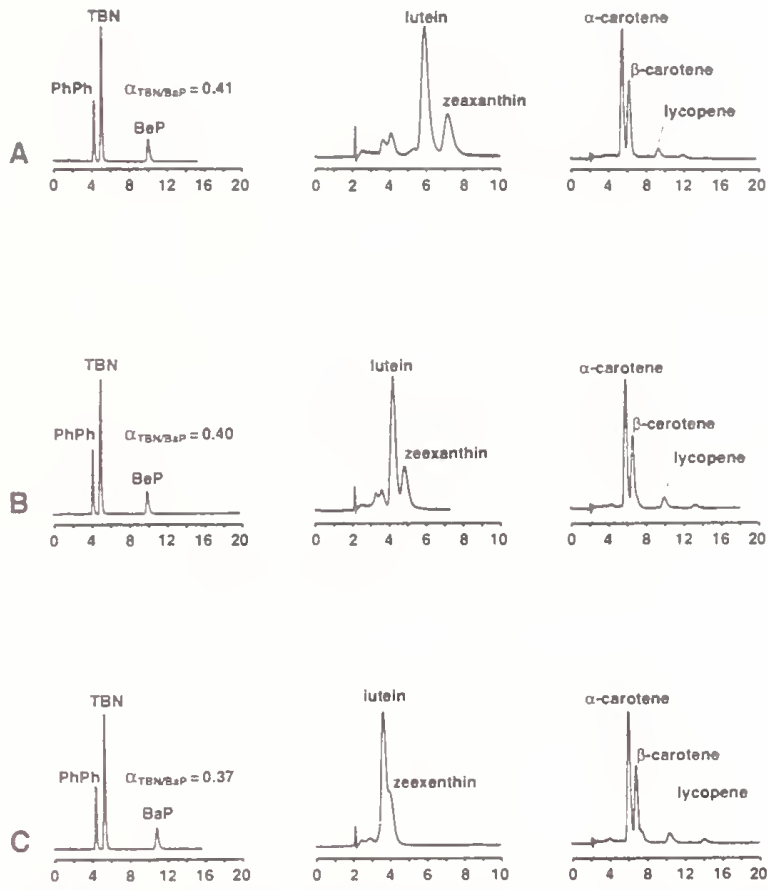

Flgure 3. Effect of endcapping for polymeric $C_{18}$ phases for polar and nonpolar solutes: (A) polymeric $C_{18}$ phase, not endcapped; (B) polymeric $C_{18}$ phase, endcapped with trimethylchlorosilane; $(C)$ polymerlc $C_{10}$ phase, endcapped with hexamethyldisllazane. Separation conditlons were as follows: left (SRM 869) see Figure 2; center (xanthophylls) $1.5 \mathrm{~mL} / \mathrm{min}$ methanol; right (carotenoids) $1.5 \mathrm{~mL} / \mathrm{min}, 90: 10$ methanol/ ethyl acetate.

investigation of phase selectivity for carotenoids indicating better separations of lutein and zeaxanthin were possible with unendcapped polymeric $\mathrm{C}_{18}$ phases. ${ }^{24}$

Stationary Phase Length. In previous studies it has been shown that shape selectivity toward PAHs is closely related to alkyl phase length. ${ }^{44}$ Better separations of isomer sets are usually achieved with long chain-length phases. This trend is observed for both monomeric and polymeric alkyl phases. For example, better shape discrimination of PAH isomers is possible with a monomeric $\mathrm{C}_{22}$ phase than with a monomeric $C_{8}$ phase. However, for any given alkyl length, polymeric phases still provide enhanced selectivity for structurally similar compounds.

It is informative to consider the size of carotenoid molecules for comparison with typical bonded phase dimensions. Thicknesses for various monomeric and polymeric alkyl phases have been measured using small angle neutron scattering (SANS), 57,58 and a summary of these results is provided in Table 2. These measurements were carried out in a reversedphase environment (methanol and deuterated methanol), giving values that have chromatographic relevance. Approximate dimensions of $\beta$-carotene were determined by molecular modeling as shown in Figure 4. Structures for all-trans conformations of octadecane and triacontane are also shown for comparison, although spectral characterization

(56) Kirkland, J. J.; Dilks, C. H.; Henderson, J. E. 17th International Symposium on Column Liquid Chromatography, May 9-14, 1993, Hamburg, Germany 1993, Abstract.

(57) Sander, L. C. Glinka, C. J.; Wise, S. A. Anal. Chem. 1990, 62, 1099-1101.

(58) Glinka, C. J.; Sander, L. C.; Wise, S. A.; Berk, N. F. Mater. Res. Soc. Symp. Proc. 1990, 166, 415-420. 
Table 2. Physkal Properlles of AlkytModilled Silica (from reference 57)

stationary phase type thickness, nm

$\mathrm{C}_{8}$ monomeric

$\mathrm{C}_{18}$ monomeric

$\mathrm{C}_{18}$ polymeric

$\mathrm{C}_{30}$ monomeric

$1.0 \pm 0.2$

$1.7 \pm 0.3$

$2.1 \pm 0.3$

$2.5 \pm 0.4$
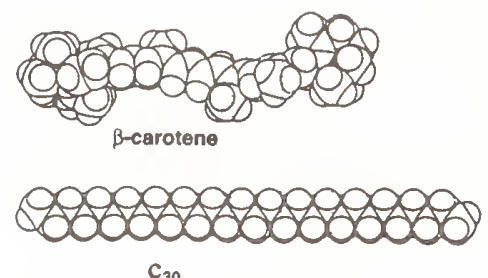

$\mathrm{c}_{30}$
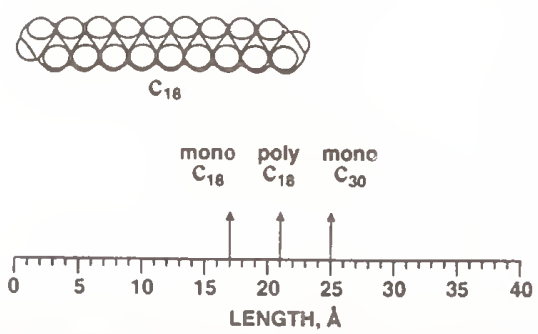

Floure 4. Space filling models for $\beta$-carotene and ail-trans hydrocarbon chains. Dotted iines indicate stationary phase thickness as determined by smaii angle neutron scattering.

studies indicate that bends and kinks in bonded alkanes reduce average phase thickness to less than if fully extended. ${ }^{57-59}$ Actual phase thicknesses as determined by SANS are indicated by dashed lines. It is clear that carotenoids are significantly larger than either monomeric or polymeric $C_{18}$ phases and closer in dimensions to the monomeric $C_{30}$ phase. The thickness of polymeric $C_{30}$ phases was not determined in the previous SANS investigation (polymeric $C_{30}$ phases had not yet been developed), but based on the measurements for monomeric and polymeric $\mathrm{C}_{18}$ phases, it is likely that a polymeric $\mathrm{C}_{30}$ phase would be slightly thicker than a monomeric $\mathrm{C}_{30}$ phase. In light of the relative dimensions of carotenoids and various alkyl phases, the potential of polymeric $\mathrm{C}_{30}$ phases was indicated for further study.

Substrate Properties. Physical and chemical properties of silica affect the chromatographic properties of the resulting bonded phase in many ways. Of course, purely mechanical aspects of the substrate such as particle size and porosity affect efficiency, backpressure, and mechanical strength, but stationary phase selectivity is also affected by such parameters as silanol activity (see discussion above) and trace metal content. ${ }^{60-65}$ To provide adequate retention of polar carotenoids in a reversed-phase system, the column to be developed should exhibit high absolute retention. Such a column would

(59) Sander, L. C.; Callis, J. B; Field, L. R. Anal. Chem. 1983, 55, 1068-1075. (60) Verzele, M.; Dewaele, C. J. Chromotogr. 1981, 217, 399-404.

(61) van den Driest, P. J.; Ritchie, H. J.; Rose, S. LC.GC 1988, 6, 124-132.

(62) Schuddinck, G.; Verzele, M. J. Chromatogr. 1987, 407, 159-169.

(63) Nawrocki, J.; Moir, D. L.; Szczepaniak, W. Chromatographia 1989, 28, 143147.

(64) Koyama, J.; Nomura, J.; Ohtsu, Y.; Nakata, O.; Takahashi, M. Chem. Lett. $1990,4,687-690$

(65) Liew, K. Y.; Tan, S. H.; Morsingh, F.; Khoo, L. E. J. Am. Oil Chem. Soc. $1982,59,480-484$.

\begin{tabular}{|c|c|c|c|}
\hline silica & $\begin{array}{l}\text { particle } \\
\text { shape }\end{array}$ & $\begin{array}{l}\text { pore diameter } \\
\text { (nm) }\end{array}$ & $\begin{array}{l}\text { surface area } \\
\left(\mathrm{m}^{2} / \mathrm{g}\right)\end{array}$ \\
\hline Kromasil 200 & spherical & $16.8^{a}$ & $196^{\circ}$ \\
\hline IMPAQ 200 & irregular & $20^{\circ}$ & $240^{a}$ \\
\hline YMC SIL200 & spherical & $20^{b}$ & $200^{b}$ \\
\hline
\end{tabular}

a Measured values. ${ }^{b}$ Nominal values reported by manufacturer.

also be advantageous for preparative separations since nonaqueous solvents would facilitate carotenoid reclamation. Yet another consideration is substrate pore diameter. It has been shown that the enhanced shape recognition exhibited with polymeric $\mathrm{C}_{18}$ phases is compromised when narrow-pore substrates less than about $15 \mathrm{~nm}$ are employed in the synthesis. ${ }^{43}$ This trend is attributed to a size exclusion effect limiting the polymeric phase synthesis, rather than a chromatographic size exclusion effect.

Each of these trends constrains the selection of a substrate with properties appropriate for the development of a "carotenoid phase". High absolute retention results from stationary phases prepared with high surface area substrates, but such substrates typically have narrow pores. Conversely, widepore silicas with pore diameters $30 \mathrm{~nm}$ or greater typically have low specific surface areas $\left(<\sim 100 \mathrm{~m}^{2} / \mathrm{g}\right)$. Three silica substrates were identified with properties intermediate to these extremes (see Table 3). Each of these materials has nominal pore diameters of $20 \mathrm{~nm}$, with moderately large specific surface areas $\left(200-240 \mathrm{~m}^{2} / \mathrm{g}\right)$. This combination of properties was anticipated to yield a chromatographic sorbent with high absolute retention and high shape recognition.

Stationary Phase Synthesis and Characterization. Polymeric $\mathrm{C}_{30}$ phases were prepared on each of the 20 -nm pore size substrates using the procedure detailed in the Experimental Section. A summary of physical characteristics of these materials is listed in Table 1. Bonded phase loadings are higher than most monomeric $\mathrm{C}_{18}$ phases but are typical of polymeric phases.

Columns were evaluated using the carotenoid mixture described previously. Many of these compounds are isomers, with structures that differ only in the location of a double bond or the position of cis-substitution. The extent of carotenoid separations varies dramatically among commercial $\mathrm{C}_{18}$ columns. As with $\mathrm{PAH}$ separations, column selectivity can be categorized broadly into monomeric and polymeric $\mathrm{C}_{18}$ groups, but differences in silanol activity cause further variations in selectivity toward xanthophylls in each of these groups. Separations of the carotenoid standards on representative commercial monomeric and polymeric $\mathrm{C}_{18}$ columns as well as the polymeric $\mathrm{C}_{30}$ column are illustrated in Figure 5. Separation conditions are the same for each column; however, a weaker mobile phase gradient was also employed for monomeric and polymeric $\mathrm{C}_{18}$ phases in an attempt to increase retention and improve separation for these columns. The longer retention times did not result in improved separation and the chromatograms are not shown. Most of the xanthophylls were separated on both the monomeric and polymeric $\mathrm{C}_{18}$ phases (Figure 5A,B); however, lutein and zeaxanthin were not resolved on the monomeric $\mathrm{C}_{18}$ phase. Differences were most apparent for the nonpolar carotenoids, which eluted 


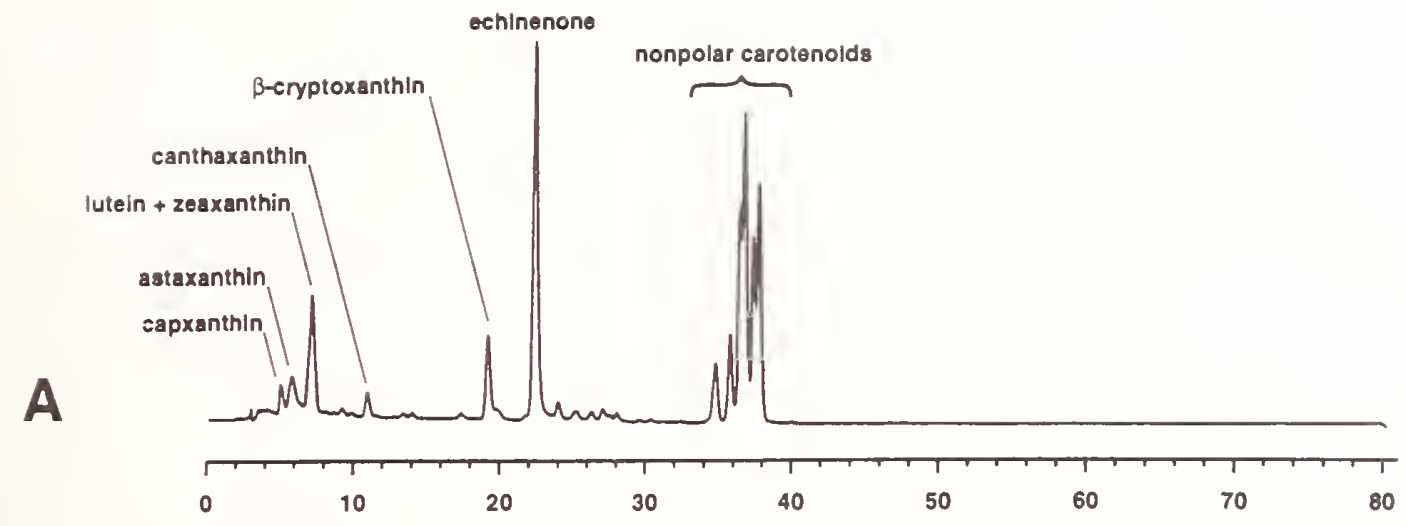

B
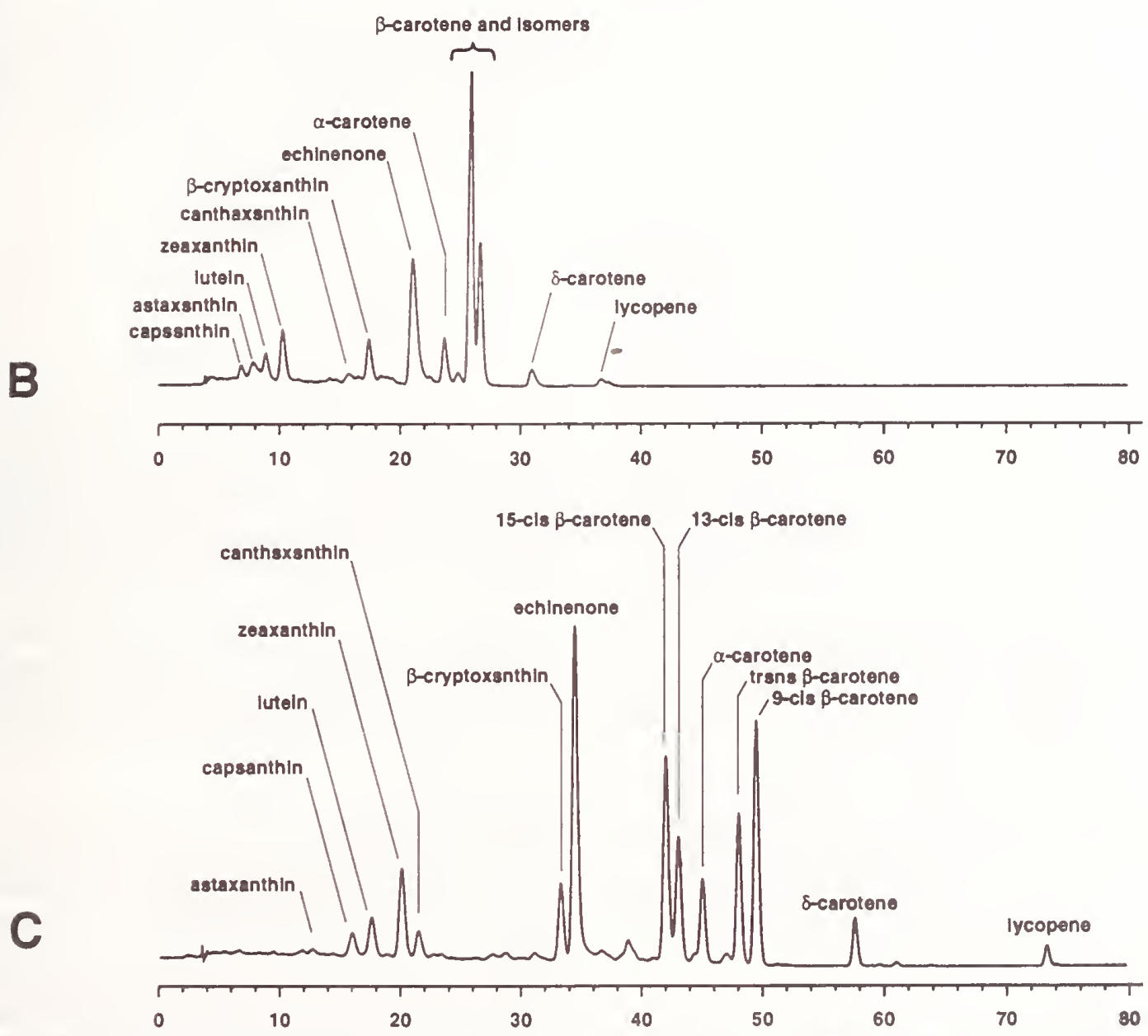

Flgure 5. Separations of carotenold standards on commercial (A) monomeric and (B) polymeric $C_{18}$ columns, as well as the englneered $C_{30}$ "carotenold column". Separation conditions were as follows: 81:15:4 to 6:90:4 methanol/methyl-tert-butyl ether (MTBE)/water over 90 min; 1 $\mathrm{mL} / \mathrm{min} ; 20^{\circ} \mathrm{C}$.

as a group with the monomeric $C_{18}$ phase and were partially separated with the polymeric $C_{18}$ phase. A better separation of both polar and nonpolar carotenoid standards was achieved using the polymeric $\mathrm{C}_{30}$ column (Figure $5 \mathrm{C}$ ). Cis-isomers of $\beta$-carotene were largely resolved from each other and from other carotenes. Better separations of the xanthophylls were also observed, including a few unidentified carotenoid impurities.

The separations illustrated in Figure 5 are not fully optimized; however, some effort was made to resolve the carotenoid standards with the polymeric $C_{30}$ column. Sepa- rations were carried out at $20^{\circ} \mathrm{C}$, and selectivity was observed to change significantly with column temperature. The separation of the xanthophylls was observed to be influenced by the amount of water in the mobile phase whereas nonpolar carotenoids were relatively insensitive to water content. Good separation of most of the carotenoid standards was possible using a nonaqueous mobile phase gradient (methanol and methyl-tert-butyl ether); however, $\beta$-cryptoxanthin and echinenone were not resolved in this environment. The choice of a linear gradient for the separations in Figure 5 was selected to facilitate column comparison and to give a simple indication 


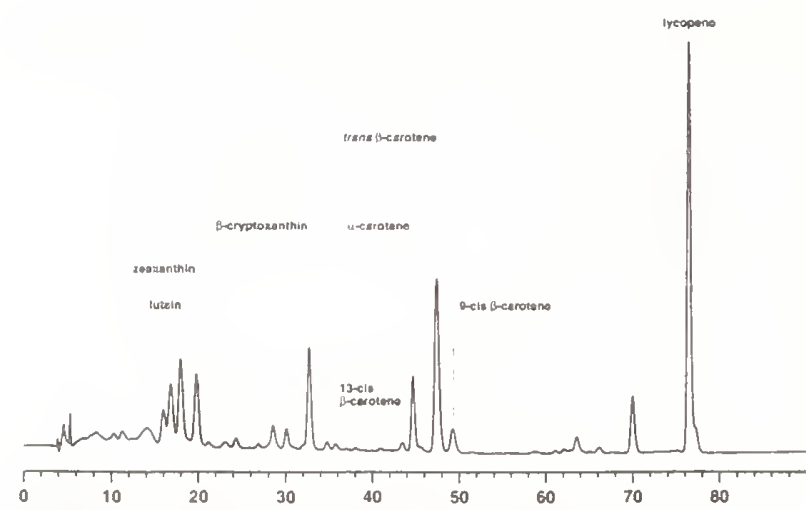

Figure 6. Separation of carotenoids from an extract of SRM 2383, Vitamins and Carotenoids in Food. For separation conditions, see Figure 5.

of overall retention behavior toward carotenoids. In practical applications separation conditions could probably be modified to reduce analysis times.

Separation of an extract of a food matrix, Standard Reference Material (candidate SRM 2383, Vitamins and Carotenoids in Food) is illustrated in Figure 6. Components were tentatively identified on the basis of retention time. Several peaks did not correspond to any of the carotene or xanthophyll standards, though it is probable that these components are isomers or oxidation products of carotenoid compounds.

To illustrate the potential of the engineered $\mathrm{C}_{30}$ carotenoid column for the separation of carotene isomers, a solution containing various $\beta$-carotene isomers was chromatographed. The sample obtained from Betatene Ltd. is prepared from algae (Dunaliella) and has high levels of cis isomers of $\beta$-carotene. A separation of this material using the "carotenoid column" is illustrated in Figure 7C. For comparison, separations of the $\beta$-carotene preparation are also shown using conventional commercial monomeric and polymeric $\mathrm{C}_{18}$ columns (parts A and B of Figure 7, respectively). Mobile phase conditions were altered in an attempt to improve separations with the monomeric and polymeric phases by increasing retention. Better separations were possible with the "carotenoid column" than with either of the $\mathrm{C}_{18}$ columns. Even so, complete separation of all of the isomers of $\beta$-carotene (and other nonpolar carotenoids) was not achieved.

\section{CONCLUSIONS}

An understanding of the cause and effect relationship between chromatographic variables and solute retention creates the potential for the development of "designer phases". For carotenoids and related compounds, molecular shape, polarity, and silanol interactions are possible parameters that can be utilized in the development of such a tailored stationary phase. Three properties have been identified as desirable for a column intended for carotenoid separations: en hanced shape recognition of isomers, high absolute retention, and silanol activity. The preparation of a column with these qualities requires the choice of an appropriate silica substrate as well
A

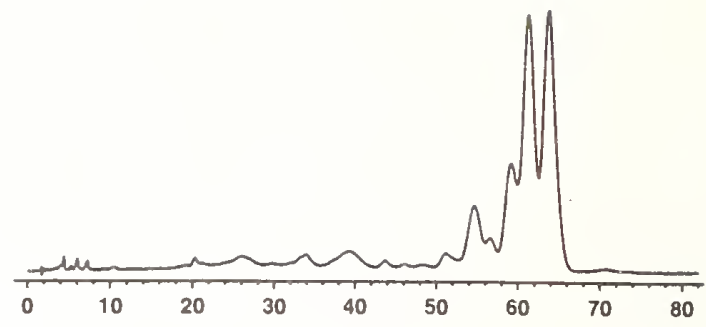

$B$

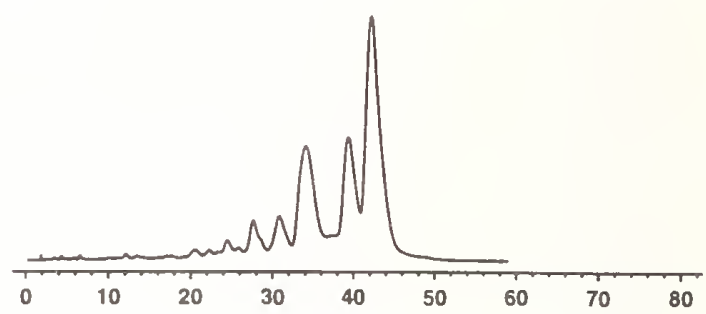

C

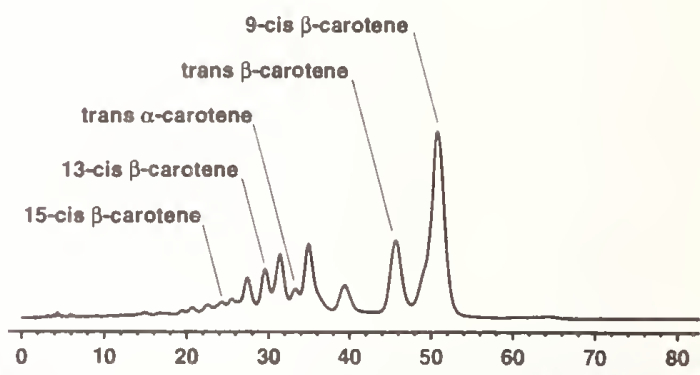

Flgure 7. Separation of a $\beta$-carotene preparation containing a high percentage of cis-isomers on commercial (A) monomeric and (B) polymeric $\mathrm{C}_{18}$ columns, and (C) "carotenold column". Separation conditions were as follows: (A and B) $3^{\circ} \mathrm{C}, 2 \mathrm{~mL} / \mathrm{min}, 90: 10$ acetonitrile/ MTBE; (C) $3^{\circ} \mathrm{C}, 2 \mathrm{~mL} / \mathrm{min}, 80: 20$ acetonitrile/MTBE.

as bonding chemistry. The use of $\mathrm{C}_{30}$ polymeric surface modification chemistry without endcapping in conjunction with a moderate pore-size, moderate surface area silica results in a bonded phase with properties well suited to the separation of carotenoids.

\section{ACKNOWLEDGMENT}

The authors thank Robert Cooley (YMC, Inc., Wilmington, NC) for gifts of YMC SIL200 silica, Neil Miller (PQ Corporation, Conshohocken, PA) for gifts of IMPAQ 200 silica, Per Möller (Eka Nobel, Bohus, Sweden) for a gift of Kromasil 200 silica, and Hemmi Bhagavan and Willy Schūep (Hoffman-LaRoche, Nutley, NJ, and Basel, Switzerland) for providing carotenoid standards. Certain commercial equipment, instruments, or materials are identified in this report to specify adequately the experimental procedure. Such identification does not imply recommendation or endorsement by the National Institute of Standards and Technology, nor does it imply that the materials or equipment identified are necessarily the best available for the purpose.

Recelved for review October 5, 1993. Accepted March 9, 1994.

- Abstract published in Advance ACS Abstracts. April 15, 1994. 


\section{DETERMINATION OF GLYCYRRHETINIC ACID}

This section describes an ion-pairing solid-phase extraction-HPLC protocol for measuring glycyrrhetinic acid (GRA) in human plasma. This method allows reliable measurements of GRA in human plasma with a detection limit of $10 \mathrm{ng} / \mathrm{mL}$ and a limit of quantification greater than or equal to $100 \mathrm{ng} / \mathrm{mL}$. The following factors are essential to this procedure.

\section{Important Factors}

\section{Calibration and Sample Preparation}

- Solutions of glycyrrhetinic acid (GRA) in polar solvents such as methanol, acetonitrile, and ethanol were found to be stable at room temperature in the dark for up to 3 weeks. However, we recommend that working standards of GRA be kept for no longer than 1 week at room temperature. We found plasma-based calibrants stored at $-70^{\circ} \mathrm{C}$ to be stable up to 4 months.

- Because of the polar nature of GRA and its affinity for plasma constituents, liquid-liquid extraction is not effective in removing GRA from the plasma matrix.

- Denaturation of the proteins, followed by ion-pairing, is crucial to obtain GRA recoveries of $>90 \%$. We use urea to denature the proteins. Both acid and base hydrolyses were tried. GRA appeared to be destroyed by the acid at the required temperature of $120^{\circ} \mathrm{C}$, while $\sim 10 \%$ of the GRA was recovered after treatment with base.

- To prevent intrusion of air bubbles into the cartridge, pass the plasma through the cartridge at a flow rate of $1-2 \mathrm{~mL} / \mathrm{min}$.

- Before eluting the GRA from the cartridge with methanol during the ion-pairing solidphase extraction (SPE) process, it is essential to wash the cartridge with 45\% methanol in water to remove unwanted constituents from the plasma matrix. Higher methanol concentrations (e.g., 50\%) will cause significant loss of GRA. 


\section{Chromatography}

- In our laboratory we use a polymeric $\mathrm{C}_{18}$ column $(0.46 \times 25 \mathrm{~cm})$. Monomeric $\mathrm{C}_{18}$ columns may also be suitable with adjustments in mobile phase composition.

- Isocratic conditions are used for this study. The mobile phase is methanol-water-acetic acid $(75: 24.7: 0.3, \mathrm{v} / \mathrm{v})$ with UV detection at $248 \mathrm{~nm}$.

For further information, please contact Jeanice Brown Thomas at (301)975-3120. 


\title{
Short Communication
}

\section{Determination of glycyrrhetinic acid in human plasma by high-performance liquid chromatography}

\author{
JEANICE M. BROWN-THOMAS*, RICHARD G. CHRISTENSEN, ROLAND RIEGER ${ }^{a}$, \\ WINFRED MALONE and WILLIE E. MAY \\ Organic Analytical Research Division. Center for Analytical Chemistry, National Institute of Siandards and \\ Technology, Gaithersburg, MD 20899 (USA)
}

(First received September 17th. 1990: revised manuscript received March 19th, 1991)

\begin{abstract}
A high-performance liquid chromatographic (HPLC) method has been developed for measuring $18 \beta$ glycyrrhetinic acid (GRA) in human plasma in the range of $0.1-3 \mu \mathrm{g} / \mathrm{ml}$. The acetate ester of GRA is added to the plasma as an internal standard, plasma proteins are denatured with urea to release GRA, and the GRA and the internal standard are extracted in an ion-pairing solid-phase extraction process. An isocratic. reversed-phase HPLC separation is used, followed by ultraviolet absorbance detection at $248 \mathrm{~nm}$. The results from the analysis of five GRA-fortified plasma pools show a mean relative standard deviation of $7 \%$ and are accurate to within $10 \%$. With evaporative concentration of the extract, the limit of detection for GRA in plasma is approximately $10 \mathrm{ng} / \mathrm{ml}$.
\end{abstract}

\section{INTRODUCTION}

Interest in investigating the cancer chemopreventive properties of herb-derived substances such as licorice root extracts of $18 \beta$-glycyrrhetinic acid (GRA; see Fig. 1) has created a need for simple, sensitive, and accurate methods for the determination of GRA in human plasma. GRA, the main metabolite of the glucoside glycyrrhizin, is obtained from licorice root, Glycyrrhiza glabra, and is believed to act as an anti-tumor agent for humans [1-3].

Only a limited number of methods for quantitative determination of GRA in human plasma have been published [4-9]. Recently, Yasuda et al. [5] described the use of ammoniacal ethanol to precipitate the proteins, followed by extraction of GRA with methylene chloride. This extraction procedure gives good recovery of GRA, approximately $90 \%$. However, the procedure requires a solvent exchange on the extract to make it compatible with reversed-phase chromatogra-

\footnotetext{
a Permanent address: Department of Analytical Chemistry, University of Ulm, Ulm. Germany.
} 
<smiles>CC1(C(=O)O)CCC2CCC3C(=CC(=O)C4C3CCC3C4CCC(O)C3(C)C)C2C1</smiles>

Glycymthetinic acid

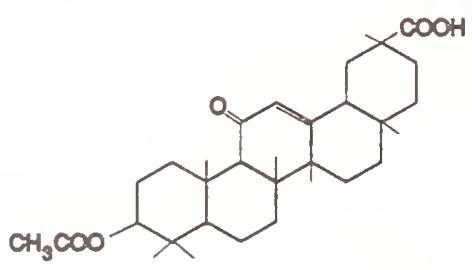

\section{Glycymhetinic acid acotate}

Fig. 1. Structures of $18 \beta$-glycyrrhetinic acid and the acetate ester of glycyrrhetinic acid.

phy. De Groot et al. [7] have reported results using a multi-dimensional ionpairing-reversed-phase chromatography scheme which requires a sophisticated valving arrangement for column switching. Most recently, Newman and Welch [8] have reported the development of a chromatographic method for determining plasma levels of GRA that uses a mixture of sodium bisulfate and sodium chloride to precipitate proteins, and acetonitrile to extract GRA. They report accurate and precise measurement capabilities, though the limit of detection is about an order of magnitude greater than the method we report here. Takeda et al. [9] have developed a reversed phase high-performance liquid chromatographic (HPLC) method that is useful in the evaluation of the pharmacokinetic profile of GRA in human serum following the oral administration of Kampo medicines containing glycyrrhizin. Their method provides a limit of detection and measurement capabilities that are comparable to those reported in our method. Other reports provided information on only qualitative measurement of GRA [10-13].

In this paper, we describe an HPLC method for the determination of GRA in human plasma. Denaturation of the plasma proteins with urea renders the GRA and the internal standard readily extractable from the matrix by ion-pairing solid-phase extraction (SPE). Analysis of the extract is by isocratic reversed-phase separation with ultraviolet (UV) absorbance detection at $248 \mathrm{~nm}$. Quantitation is accomplished through use of an internal standard, the acetate ester of GRA (see Fig. 1), which is not commercially available, but can be easily prepared. This method allows reliable measurements of GRA in human plasma with a detection limit of $10 \mathrm{ng} / \mathrm{ml}$ and with reliable quantitative measurements being possible at greater than or equal to $100 \mathrm{ng} / \mathrm{ml}$. 


\section{EXPERIMENTAL ${ }^{a}$}

\section{Standards and materials}

Standard solutions with concentrations ranging from 0.1 to $10 \mu \mathrm{g} / \mathrm{ml}$ GRA (Sigma, St. Louis, MO, USA) and from 3 to $7 \mu \mathrm{g} / \mathrm{ml} \mathrm{GRA} \mathrm{acetate} \mathrm{were} \mathrm{prepared}$ (synthesis described below) in methanol and in plasma. Working standards were prepared from the methanol solutions for use in calibration and were run prior to sample analysis to determine retention times and detector response factors. The purities of the individual reference compounds were determined by direct injection of the respective reference solutions into the chromatograph, using the HPLC separation described below.

Tetrabutylammonium phosphate (TBAP; Waters Assoc., Milford, MA, USA) was used as the ion-pairing reagent. SPE cartridges ( $\mathrm{C}_{18}$ Sep-Pak, Waters Assoc.) were used for extraction. The heparin-treated plasma used for this work was a commercial product that had been tested for hepatitis-B and HIV virus.

\section{Synthesis of GRA acetate}

GRA acetate (internal-standard) was prepared by mixing $2 \mathrm{~g}$ of GRA, $15 \mathrm{ml}$ of acetic anhydride, and $30 \mathrm{ml}$ of pyridine. The mixture was allowed to react overnight and was then poured into cold water. The GRA acetate precipitated, was removed by filtration, and was then further purified by recrystallization from $n$-propanol.

\section{Sample extraction}

To $1 \mathrm{ml}$ of plasma, $0.5 \mathrm{~g}$ of urea, $3 \mu \mathrm{g}$ of the internal standard in methanol, and $200 \mu \mathrm{l}$ of $0.5 M$ TBAP in water were added. The solution was vortex-mixed for 1 min prior to extraction to ensure proper mixing. The $\mathrm{pH}$ of the resulting solution was about 10 .

A $\mathrm{C}_{18}$ SPE cartridge, conditioned sequentially with $3 \mathrm{ml}$ each of methanol, water, and $0.5 \mathrm{mM}$ TBAP in water, was used to extract the GRA from the plasma matrix. The 1-ml plasma sample was passed through the cartridge at a rate of about $1 \mathrm{ml} / \mathrm{min}$. After washing the cartridge with $3 \mathrm{ml}$ of $5 \mathrm{mM}$ TBAP in water and $3 \mathrm{ml}$ of $45 \%$ methanol in water to remove unwanted constituents, the GRA and internal standard were eluted with $2 \mathrm{ml}$ of methanol. This eluate was concentrated under a stream of nitrogen to $1 \mathrm{ml}$ or less, depending on the sensitivity required.

a Certain commercial products are identified to specify adequately the experimental procedure. Such identification does not imply endorsement or recommendation by the National Institute of Standards and Technology, nor does it imply that the materials identified are necessarily the best available for the purpose. 


\section{HPLC apparatus and conditions}

The chromatograph used in this work consisted of a single-piston solvent delivery system, a variable wavelength UV-VIS detector, a data system, and an autosampler. The 5- $\mu \mathrm{m}$ (30-nm-pore) polymerically bonded octadecylsilane column $(25 \mathrm{~cm} \times 0.45 \mathrm{~cm} \mathrm{I.D.,} \mathrm{Vydac} \mathrm{TP)} \mathrm{was} \mathrm{protected} \mathrm{with} \mathrm{a} \mathrm{direct-connect}$ pre-column that was packed with $10-\mu \mathrm{m} \mathrm{C}_{18}$ particles of similar type. Both columns were obtained from The Separations Group (Hesperia, CA, USA). Column temperature was $28^{\circ} \mathrm{C}$. Injection volume was $30 \mu \mathrm{l}$. The mobile phase was methanol-water-acetic acid (75:24.7:0.3, v/v), at a flow-rate of $2 \mathrm{ml} / \mathrm{min}$. UV absorbance detection at $248 \mathrm{~nm}$, the absorption maximum of GRA, was used for monitoring the effluent. Analyte concentrations were calculated using peak-area ratios of GRA and internal standard.

\section{RESULTS AND DISCUSSION}

In the development of this method, preliminary studies were made to identify an appropriate internal standard, to investigate the stability of GRA in various solvents under feasible storage conditions, to optimize the extraction of GRA from the plasma matrix, and to develop a chromatographic system for separating GRA and GRA acetate and matric interferences.

\section{Internal standard}

The required properties of an internal standard for this analysis are that it be a sufficiently close homologue of the analyte so that it would mimic the extractability of the analyte from the matrix and that it would be readily separable from the analyte by HPLC. The acetate ester of GRA meets these requirements, and, although not commercially available, is easily prepared with common reagents, as described earlier.

\section{Stability of GRA standard solutions}

To verify the stability of GRA in various solvents, solutions at approximate . concentrations of $10 \mu \mathrm{g} / \mathrm{ml}$ were prepared in methanol, acetonitrile, and ethanol, both with and without the addition of 2,6-di-tert.-butyl-4- methylphenol (BHT) as an antioxidant. These were stored at room temperature in glass-stoppered flasks wrapped in foil to exclude light. The UV absorbance at $248 \mathrm{~nm}$ was monitored for three weeks and no significant changes were observed in solutions with or without BHT. No extraneous peaks appeared upon HPLC analysis of aliquots of these solutions, further confirming that no degradation occurred. Repeated analyses of plasma-based calibrants kept frozen at $-70^{\circ} \mathrm{C}$ showed these materials to be stable over a four-month period.

\section{Extraction of GRA from plasma}

It is difficult to quantitatively extract GRA from the plasma matrix because of 
its polar nature and its affinity for plasma constituents. In evaluating extraction alternatives, acidic $(\mathrm{pH} 3)$ and basic $(\mathrm{pH} \mathrm{10)}$ aqueous solutions of GRA were extracted with butanol, methylene chloride, and hexane. Butanol and methylene chloride were effective extractants, but hexane was not, suggesting that SPE using $n$-alkane bonded phases would require ion-pairing agents to be effective.

We found that ion-pairing with $\mathrm{C}_{18}$ SPE quantitatively extracted GRA from aqueous solutions, while recoveries from plasma were only $60-70 \%$. This indicated that the loss was not due to solubility considerations, but due to the binding of GRA to plasma constituents. When urea was used to denature the proteins, recoveries of both GRA and GRA acetate were $>90 \%$. Both acid and base hydrolyses were also investigated as means for denaturing plasma proteins. GRA appeared to be destroyed by acid at the required temperature $\left(120^{\circ} \mathrm{C}\right)$ and only approximately $10 \%$ of the GRA was recovered after treatment with base.

To obtain quantitative recovery in the SPE extraction, it was necessary to pass the pre-treated plasma slowly through the cartridge and to prevent intrusion of air bubbles into the cartridge. A flow-rate of $1-2 \mathrm{ml} / \mathrm{min}$ was suitable. An intermediate wash with $45 \%$ methanol in water was employed to elute interfering components from the plasma while retaining GRA and GRA acetate in the cartridge. The use of higher methanol concentrations, e.g. 50\%, results in significant loss of GRA.

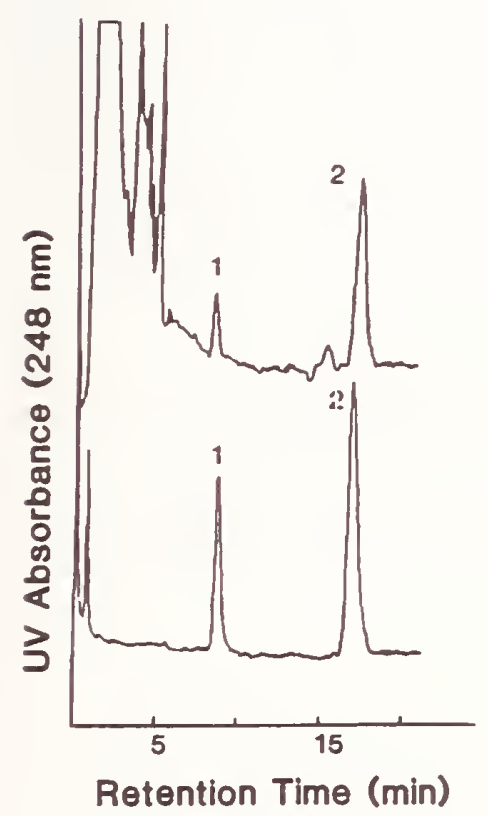

Fig. 2. Lower trace: HPLC from the analysis of a standard solution of (1) $1 \mu \mathrm{g} / \mathrm{ml} \mathrm{GRA}$ and (2) $3.4 \mu \mathrm{g} / \mathrm{ml}$ GRA acetate using methanol-water-acetic acid $(75: 24.7: 0.3, \mathrm{v} / \mathrm{v})$ at a flow-rate of $2 \mathrm{ml} / \mathrm{min}$ on a $\mathrm{C}_{18}$ column with UV detection at $248 \mathrm{~nm}$. Responses shown represent $30 \mathrm{ng}$ GRA and $90 \mathrm{ng}$ GRA acetate. Upper trace: HPLC from the analysis of plasma extract using solid-phase extraction: (1) $0.38 \mu \mathrm{g} / \mathrm{ml}$ GRA and (2) $2.9 \mu \mathrm{g} / \mathrm{ml} \mathrm{GRA}$ acetate. 


\section{Chromatographic separation}

A chromatographic separation that resolves GRA and the internal standard from one another, and from potentially interfering plasma components, is easily achieved through use of a mobile phase consisting of methanol-water-acetic acid $(75: 24.7: 0.3, \mathrm{v} / \mathrm{v})$. Acetic acid is added only to effect ionization suppression. Though we used a polymerically bonded $C_{18}$ column [14,15], monomerically bonded $\mathrm{C}_{18}$ columns [15] would probably serve as well with suitable adjustments in the mobile phase. Chromatograms of a plasma extract and a GRA solution in methanol using the SPE-HPLC protocol is illustrated in Fig. 2. The elution time of GRA is $8.5 \mathrm{~min}$ and of the internal standard is $16.7 \mathrm{~min}$.

\section{Quantification of GRA}

Calibration curves were established from both plasma and methanol solutions over the range $0.1-3 \mu \mathrm{g} / \mathrm{ml}$. Linear relationships between concentration and the ratio of peak areas for GRA and the internal standard were obtained for both plasma- and solution-based standards. The co-linearity of these calibration curves demonstrates that the method is free of matrix effects. Thus, for convenience, we used solution-based standards for routine calibration.

Plasma samples that contained GRA at concentrations ranging from 0.1 to 3 $\mu \mathrm{g} / \mathrm{ml}$ were analyzed using the SPE-HPLC procedure. At least two aliquots from each plasma sample were extracted, and duplicate HPLC injections were made from each extract.

The results of this study are shown in Table I. Within-day precision is good, with relative standard deviations ranging from 4 to $7 \%$. No significant betweenday variability was observed. Agreement with the known values is within $10 \%$. The limit of detection of GRA in plasma using our method is about $10 \mathrm{ng} / \mathrm{ml}$, which corresponds to a limit of quantification of approximately $100 \mathrm{ng} / \mathrm{ml}$.

The procedure we have described has been successfully implemented for our

\section{TABLE I}

HPLC MEASUREMENTS OF 18 $\beta$-GLYCYRRHETINIC ACID IN PLASMA AFTER ION-PAIRING SOLID-PHASE EXTRACTION

Two aliquots of each plasma sample were extracted and analyzed in duplicate by HPLC. Solution-based calibrants were used for these analyses.

\begin{tabular}{lll}
\hline $\begin{array}{l}\text { Known } \\
\text { concentration } \\
(\mu \mathrm{g} / \mathrm{ml})\end{array}$ & $\begin{array}{l}\text { Mean found } \\
\text { concentration } \\
(\mu \mathrm{g} / \mathrm{ml})\end{array}$ & $\begin{array}{l}\text { Relative } \\
\text { standard deviation } \\
(\%)\end{array}$ \\
\hline 0.104 & 0.104 & 6 \\
0.520 & 0.571 & 10 \\
0.966 & 1.03 & 7 \\
1.90 & 1.74 & 4 \\
2.74 & 3.04 & 7 \\
\hline
\end{tabular}


value assignment of quality control materials distributed to investigators involved in National Cancer Institute-supported pre-clinical trials. It provides a facile, sensitive, precise, and sufficiently accurate means for laboratory use in investigative studies to determine subject compliance in licorice root-dosing studies and for evaluating the efficacy of GRA as a chemopreventive agent.

\section{ACKNOWLEDGEMENTS}

The authors would like to acknowledge partial financial support for this research from the Division of Cancer Prevention and Control of the National Cancer Institute (USA) under interagency agreement Y01-CN-80655.

\section{REFERENCES}

1 Y. Kiso, M. Tohkin, H. Hikino, M. Hattori, T. Sakamoto and T. Namba, Planta Med., 50 (1984) 298-302.

2 Y. L. Liu, B. D. Roebuck, J. D. Yager, J. D. Groopman and T. W. Kensler, Toxicol. Appl. Pharmacol., 93 (1988) 442-45I.

3 M. H. Davies, Diss. Abstr. Intr. B, 48 (1988) 2275-2500.

4 T. J. F. Savelkoul and P. van Volten, J. Chromalogr., 456 (1988) 71-81.

5 K. Yasuda, T. Shibuya, M. Nozaki, K. Tsurumi, H. Fujimura and F. Kaneuchi, Zasshi Yakagaku, 98 (1978) 1545-1547.

6 H. Hughes and R. J. Cowles, N.Z. Med. J., 85 (1977) 398.

7 G. de Groot, R. Koops, E. A. Hogendoorn, C. E. Goewie, T. J. F. Savelkoul and P. van Vloten, J. Chromatogr., 456 (1988) 71-81.

8 R. A. Newman and M. Welch, J. Liq. Chromatogr., 13 (1990) 1585-1594.

9 S. Takeda, O. Hiromasa, Y. Wakui, A. Asami, Y. Matsuzaki, H. Sasaki, M. Aburada and E. Hosoya, J. Chromalog :, 530 (1990) 447-451.

10 L. E. Carlat, H. W. Margraf, H. H. Weathers and T. E. Weichselbaum, Proc. Soc. Biol. Med., 102 (1959) 245-250.

11 J. Killacky, M. S. F. Ross and T. D. Turner, Planta Med., 30 (1976) 310-316.

12 D. V. Parke, S. Pollock and R. T. Williams, J. Pharm. Pharmacol., 15 (1963) 500-506.

13 D. V. Parke, T. C. Hunt and P. Iveson, Clin. Sci., 43 (1972) 393-400.

14 L. C. Sander and S A. Wise, Anal. Chem., 56 (1984) 504-510.

15 L. C. Sander and S. A. Wise, in M. W. Cooke and A. J. Dennis (Editors), Polynuclear Aromatic Hydrocarbons: Mechanisms, Methods, and Metabolism, Battelle Press, Columbus, OH, 1983, pp. 11331144. 


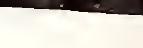




\section{MEASUREMENT OF OLTIPRAZ IN SERUM}

This section describes three reversed-phase liquid chromatographic methods for the determination of Oltipraz in serum. Two methods employ off-line solid-phase extraction, one using a labeled internal standard suited to thermospray mass spectrometric detection and the other using a homologous internal standard for use with optical absorbance detection. The third method includes an on-line solid-phase extraction step and does not require internal standards. Detection limits are ca. $1 \mathrm{ng} / \mathrm{mL}$ of serum. Listed below are the more important factors related to this analysis.

\section{Important Factors}

\section{Calibration and Sample Preparation}

- Oltipraz and related compounds are light-sensitive. Store solutions in the dark when not in use.

- Aqueous standard solutions are unstable in storage presumably owing to sorption to the container. Create standards daily by diluting alcoholic stock solutions.

- Recovery in solid-phase extraction is enhanced by diluting serum samples three- or fourfold with water.

- Evaporation of extracts to dryness causes serious losses of Oltipraz. Evaporate to 100$200 \mu \mathrm{L}$ and inject.

- Serum samples containing Oltipraz are not stable in long storage, even at $-80{ }^{\circ} \mathrm{C}$. Limit such storage to 1 or 2 months for best results.

\section{Chromatography}

- Filtration of extracts can cause losses of Oltipraz owing to sorption on the filter. Use a pre-column as a bed filter to retain injected particulate matter.

- Buffer in the mobile phase (e.g., ammonium acetate) will often sharpen the peaks and stabilize the retention times. 
- Several types of $\mathrm{C}_{18}$ columns were tried and all were suitable once the mobile phase was adjusted to the proper methanol concentration.

- Oltipraz has absorbance maxima at 232,305 , and $450 \mathrm{~nm}$. The extinction coefficients are $10,800,13,800$, and $8,100 \mathrm{~L}^{*} \mathrm{~mol}^{-1} * \mathrm{~cm}^{-1}$, respectively. Detection at $450 \mathrm{~nm}$ is preferred to minimize interferences from serum components.

For further information, please contact Richard Christensen at (301)975-3129. 


\title{
Determination of Oltipraz in serum by high-performance liquid chromatography with optical absorbance and mass spectrometric detection
}

\author{
Richard G. Christensen \\ Chemical Science and Technology Laboratory, National Institute of Standards and Technology, Gaithersburg, MD 20899 (USA)
}

\section{Winfred Malone}

Chemoprevention Branch, Division of Cancer Prevention and Control, National Cancer Institute, National Institutes of Health. Bethesda. MD 20892 (USA)

(First received July 14th, 1992; revised manuscript received September 29th, 1992)

\begin{abstract}
Three methods have been developed for the analysis of Oltipraz in serum. A method suitable for routine use employs spiking with a homologous internal standard, off-line solid-phase extraction, high-performance liquid chromatographic separation, and optical absorbance detection at $450 \mathrm{~nm}$. Method detection limit is about $1 \mathrm{ng} / \mathrm{ml}$. A second method, less susceptible to bias from co-eluting interferences, uses a stable isotope-labeled internal standard. similar extraction and separation, and detection by thermospray mass spectrometry. Method detection limit is about $0.2 \mathrm{ng} / \mathrm{ml}$. A third method was developed which can be used without specially synthesized internal standards. It uses on-line solid-phase extraction, with quantification by comparison with external standards. Method detection limit is about $3 \mathrm{ng} / \mathrm{ml}$. Good agreement was observed between these methods and with similar and different methods run in other laboratories. Calibration curves were linear over the entire range which was investigated, i.e., up to $500 \mathrm{ng} / \mathrm{ml}$. Coefficients of variation were similar for all three methods, being about $5 \%$.
\end{abstract}

\section{INTRODUCTION}

Oltipraz, 4-methyl-5-(2-pyrazinyl)-1,2-dithiole-3-thione, is a synthetic dithiolethione that has been found to have chemoprevention activity in breast, skin, lung, and bladder cancer animal models. Epidemiological studies have also reported that consumption of certain vegetables that are known to contain dithiolethones, namely

Correspondence 10: Richard G. Christensen, National Institute of Standards and Technology, Chemical Science and Technology Laboratory, Organic Analytical Research Division, 222/ B158, Gaithersburg, MD 20899, USA. cauliflower, brussels sprouts, and cabbage, have been associated with decreased cancer risk in humans [1]. These chemopreventive efficacy studies, together with relatively low toxicity of Oltipraz as observed thus far in animals, makes it an important compound for continued investigation as a cancer preventive agent.

This study reports on methods for measuring Oltipraz in human serum, a necessary initial step for qualifying a compound for entrance into human clinical trials. The National Institute of Standards and Technology (NIST) is responsible for providing assistance to grantee laboratories of the Division of Cancer Prevention and Con- 
trol, National Cancer Institute, in the areas of analytical method development and quality assurance. The quality assurance approach consists of circulating performance-evaluation standards to the laboratories to identify problem areas, and when problems are discovered, to provide counseling in method refinement and trouble-shooting. The development of several analytical methods for assaying serum levels of Oltipraz was necessary in the course of preparing for this program. One or more routine methods suitable for use in the clinical laboratories were needed, as well as one or more reference methods.

Solid-phase extraction of Oltipraz from serum samples using silica modified with octadecylsilane $\left(\mathrm{C}_{18}\right)$ proved to be quite effective, although another laboratory [2] reports the successful use of liquid-liquid extraction. Two approaches were used. The first, an off-line extraction using commercial solid-phase extraction cartridges, requires the use of an internal standard for volume correction and to compensate for non-quantitative recovery. It is desirable that the internal standard used should be chemically similar to Oltipraz, so that the extraction recovery and detector response will be about the same. This extraction technique was used with both optical absorbance detection and mass spectrometric (MS) detection.

Since the internal standards used with the extraction technique above are not commercially available, an on-line solid-phase extraction was also developed. A method based on this extraction, while somewhat cumbersome to perform with non-automated equipment, offers the possibility of doing the analysis without reference to an internal standard. It also offers some additional method independence, since the extract concentration steps are eliminated. The use of independent methods is an important means for discovering the presence of (and sources of) analytical bias.

Separation of Oltipraz from other extracted serum components was readily effected by reversed-phase chromatography on silica bonded with octadecylsilane, using a methanol-water mobile phase. Oltipraz has strong optical absor- bances at 232, 305, and $450 \mathrm{~nm}$. These make it quite amenable to determination by high-performance liquid chromatography with absorbance detection (LC-UV) at the levels likely to be found in serum from the NCI studies, i.e., 10-500 $\mathrm{ng} / \mathrm{ml}[2]$.

Analytical determination by LC-MS is also possible, since Oltipraz provides a good yield of negatively charged molecular ions, $\mathrm{M}^{-}$, in a thermospray interface with discharge-assisted ionization. We have found that a deuterated internal standard gives both the desired co-elution and similarity of ionization mechanism for isotope dilution analysis.

\section{EXPERIMENTAL}

\section{LC-UV method with homologous internal stan-} dard

The internal standard used in this analysis is 4-ethyl-5-(2-pyrazinyl)-1,2-dithiole-3-thione $(\text { I.S. })^{a}$, a homolog of Oltipraz which was synthesized in this laboratory. It has the desirable attributes of being chemically similar to Oltipraz, so that its extraction recovery should be the same, and also of having similar optical absorptivity, so that it can be used at about the same concentration as the Oltipraz.

Standard solutions were prepared by dispensing appropriate aliquots of a stock solution of Oltipraz (Rhone-Poulenc, Vitry, France) in absolute ethanol (Warner-Graham, Cockeysville, $\mathrm{MD}, \mathrm{USA}$ ) into $50-\mathrm{ml}$ volumetric flasks. These were then made up to volume with water. Concentrations ranged between 10 and $500 \mathrm{ng} / \mathrm{ml}$. A solution of I.S. was also made up in ethanol at about $2 \mu \mathrm{g} / \mathrm{ml}$. This was used to spike both the standard solutions and samples. If the same I.S. solution is used to spike both the standards and the serum samples, the accuracy of its concentration is not critical.

Oltipraz in solution is subject to photodegradation, so that flasks should be covered, wrapped with foil, or kept in the dark when not actually in

\footnotetext{
${ }^{a}$ The author will make small quantities of these internal standards available to qualified investigators.
} 
use. For storage, ethanol solutions kept in a refrigerator have been found to be stable for up to two weeks. The aqueous standard solutions are subject to possible sorptive losses of Oltipraz, and therefore should be made up fresh daily.

A calibration curve was constructed by placing $1-\mathrm{ml}$ aliquots of standard solutions in vials and spiking with $50 \mu \mathrm{l}$ of I.S. solution. The aliquots were then evaporated to $100-200 \mu$, either by blowing down with inert gas or by the use of a centrifugal vacuum evaporator (SVC-100H, Savant Instruments, Farmingdale, NY, USA) ${ }^{a}$. The concentrates were injected into the chromatograph without further treatment. Subjecting them to the same extraction-concentration process that was used with the serum samples gave identical results.

For extraction of Oltipraz from serum, l-ml aliquots of serum were placed in vials and spiked with $50 \mu \mathrm{l}$ of I.S. solution. The serum samples were then diluted with about $3 \mathrm{ml}$ of water. This dilution has been found to increase the recovery. Sep-Pak solid-phase extraction cartridges (Waters Assoc., Milford, MA, USA) packed with $\mathrm{C}_{18}$ modified silica were conditioned with several milliliters of methanol followed by $2 \mathrm{ml}$ of water. The spiked, diluted serum was then passed through at the rate of about $2 \mathrm{ml} / \mathrm{min}$. It is important to keep the cartridge packing wet and not allow air bubbles to enter, except as specified later. Each cartridge was then washed with $2 \mathrm{ml}$ of water and $2 \mathrm{ml}$ of $40 \%(\mathrm{v} / \mathrm{v})$ methanol in water. These washings remove many of the unwanted serum components. The last washing was expelled with $3 \mathrm{ml}$ of air. The Oltipraz was then eluted with $2 \mathrm{ml}$ of methanol followed by $3 \mathrm{ml}$ of air. This eluate was concentrated by the same process used with the standard solutions. The eluate contained some water and, after evaporative concentration, the water concentration was high enough that large volumes could be injected

\footnotetext{
a Certain commercial products are identified in order to adequately specify the experimental procedure. This does not imply recommendation or endorsement by the National Institute of Standards and Technology, nor does it imply that the material or equipment identified as necessarily the best available for the purpose.
}

without degradation of chromatographic peak shapes.

For the LC separations, a mobile phase of 2:1 $(\mathrm{v} / \mathrm{v})$ methanol-water containing $0.05 \mathrm{~mol} / \mathrm{l} \mathrm{am}$ monium acetate was used. Solvent delivery was by an ISCO M2350/2360 pumping system (ISCO, Lincoln, NE, USA). Flow-rates of 1.5-2.0 $\mathrm{ml} / \mathrm{min}$ were satisfactory. The buffer served to sharpen up some of the peaks from serum components which might otherwise interfere with the Oltipraz or internal standard peaks. The injector was fitted with a 1-ml loop, arranged so that liquid injected into the loop is back-flushed into the column. A pre-column of $50 \mu$ l capacity (Upchurch Scientific, Oak Harbor, WA. USA), filled with $\mathrm{C}_{18}$ Corasil (Waters Assoc.) packing supported on $0.5-\mu \mathrm{m}$ frits, was used to protect the 25 $\mathrm{cm} \times 4.6 \mathrm{~mm}$ Vydac $5-\mu \mathrm{m} \mathrm{C}_{18}$ column (The Separations Group, Hesperia, CA, USA). This precolumn served not only to remove components which might irreversibly sorb to the analytical column, but also acts as a filter bed. The concentrated extracts often contained visible particulate matter, but off-line pre-filtration caused serious losses of Oltipraz.

Detection was by optical absorbance at $450 \mathrm{~nm}$ using an ISCO V4 variable-wavelength UV-VIS detector (ISCO), with sensitivity set at 0.005 or 0.010 absorbance units full scale. If an absorption maximum at 232 or $305 \mathrm{~nm}$ is used for detection, the sensitivity is 33 or $70 \%$ greater, respectively, but there is a greater risk of interference from the absorbances of co-eluting serum components.

Peak heights were measured for quantification,

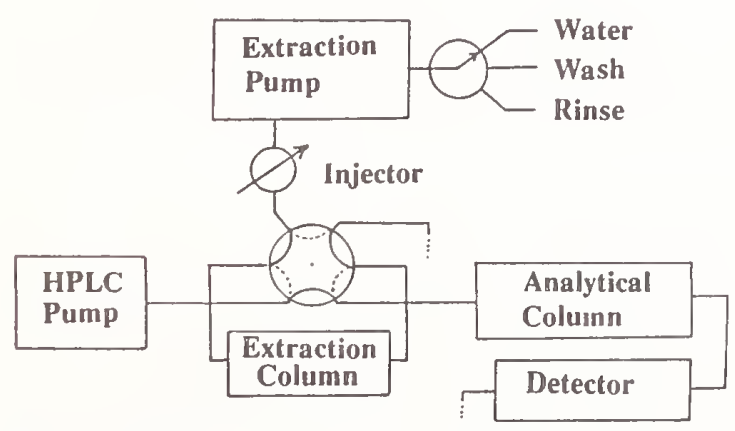

Fig. 1. Schematic of the on-line extraction system. 
though measurements of peak areas would also serve. A calibration curve was constructed by plotting the ratio, $R$ (where $R=$ Oltipraz response/I.S. response), versus the Oltipraz concentration in the standard solution. The plot was found to be linear over the range of interest, i.e., $10-500 \mathrm{ng} / \mathrm{ml}$. Oltipraz concentrations in serum samples could then be inferred by comparing the value of the ratio, $R$, for each sample to the calibration curve.

\section{LC-UV method with external standard}

An additional HPLC pump (M6000, Waters Assoc.) was used to pump the samples (or standards) and washing solutions through an extraction column which was substituted for the injection loop of the HPLC system, as shown in Fig. 1. A $40 \mathrm{~mm} \times 4.6 \mathrm{~mm}$ I.D. extraction column was constructed and packed with $10-\mu \mathrm{m} \mu$ Bondapak $\mathrm{C}_{18}$ (Waters Assoc.). This packing is somewhat less retentive than the Vydac $\mathrm{C}_{18}$ analytical column, so that some focusing of the extracted Oltipraz will occur when it is displaced onto the analytical column.

Serum samples and standard solutions were diluted 1:4 with water prior to injection. With water flowing through the extraction column at 2 $\mathrm{ml} / \mathrm{min}, 1.00 \mathrm{ml}$ of diluted serum or standard solution was injected. After the passage of $5 \mathrm{ml}$ of water, $5 \mathrm{ml}$ of washing solution containing $35 \%$ methanol in water was pumped through. The extraction column was then switched in line with the analytical column, whereupon the extracted materials were eluted. The extraction column was conditioned for the next extraction by pumping through $10 \mathrm{ml}$ of absolute ethanol, followed by $10 \mathrm{ml}$ of water. For quantification, peak heights from the serum samples were compared to the peak heights resulting from injections of standard solutions.

\section{LC-MS method}

A Vestec Model 201 thermospray LC-MS system (Vestec, Houston TX, USA) was used as the detector for isotope dilution determinations. The I.S. used was 4-(methyl-d3)-5-(2-pyrazinyl)-1,2dithiole-3-thione (Oltipraz-d3), which was syn- thesized in this laboratory. Standard solutions, spiking, extraction, and concentration steps were similar to those used for the LC-UV method. The mobile phase was the same except that the ammonium acetate was omitted because it yielded a background ion at $m / z 229$, interfering with the ions from the internal standard. The flow-rate used was $1.00 \mathrm{ml} / \mathrm{min}$. Obviously, the on-line extraction described above can also be used in this method, with the addition of Oltipraz-d3 as I.S.

The source block was operated at $275^{\circ} \mathrm{C}$, and the vaporizer was operated near the "take-off" temperature, giving a tip temperature of about $210^{\circ} \mathrm{C}$, and a vapor temperature of about $240^{\circ} \mathrm{C}$. Discharge-assisted ionization was used, and the $\mathrm{M}^{-}$ions of Oltipraz and Oltipraz-d3 were monitored at $m / z 226$ and 229, respectively. A calibration curve was constructed by the same method employed for LC-UV quantification. As a check on the integrating algorithm, calculations have been made using both peak areas and peak heights, with no significant difference appearing in the results.

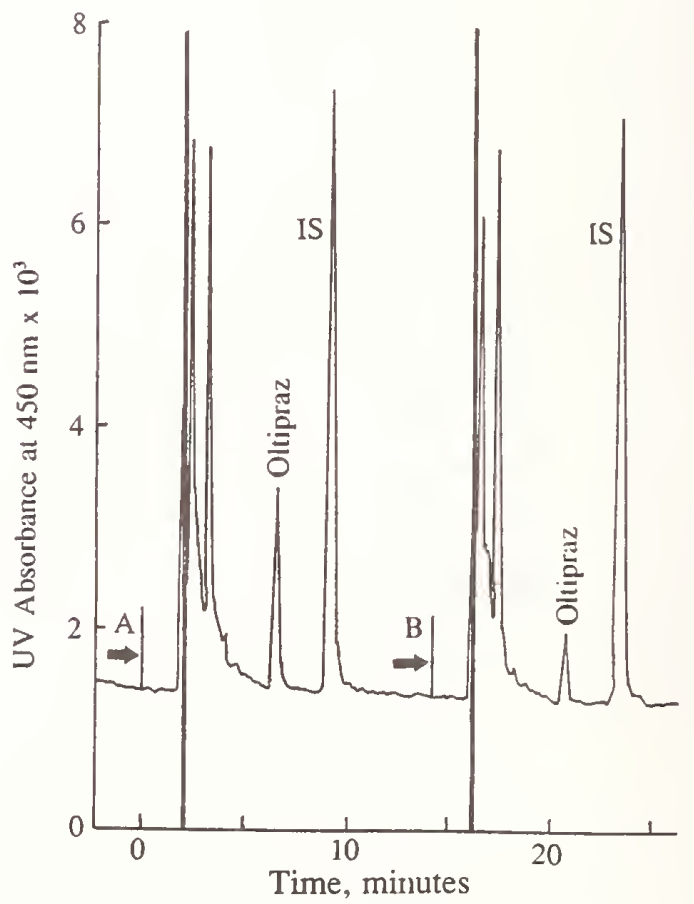

Fig. 2. Chromatograms of serum extracts. (A) Injection of the extract from a sample containing $47.7 \mathrm{ng} / \mathrm{ml}$ Oltipraz, spiked with $150 \mathrm{ng} / \mathrm{ml} \mathrm{I.S.} \mathrm{(B)} \mathrm{Injection} \mathrm{of} \mathrm{the} \mathrm{extract} \mathrm{from} \mathrm{a} \mathrm{sample}$ containing $20.2 \mathrm{ng} / \mathrm{ml} \mathrm{Oltipraz,} \mathrm{spiked} \mathrm{with} 150 \mathrm{ng} / \mathrm{ml} \mathrm{I.S.}$ 


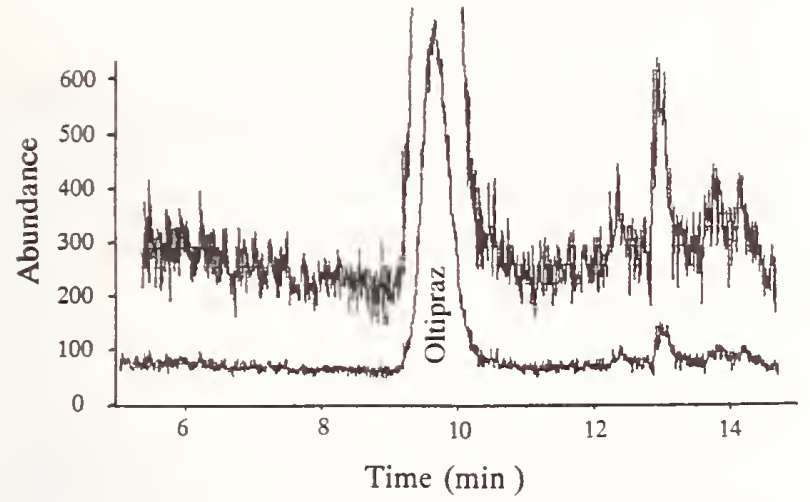

Fig. 3. Single-ion monitoring of the $\mathrm{M}^{-}$ion of Oltipraz ( $m / 2$ 226) from the injection of a serum extract containing about $1 \mathrm{ng}$ of Oltipraz. The upper trace is recorded at $5 \times$ higher sensitivity.

\section{RESULTS AND DISCUSSION}

Recoveries observed in the methods using offline extraction ranged between 50 and $90 \%$. The losses appear to occur during the concentration of the extracts, rather than being due to inefficiency of extraction. Whether the losses are caused by oxidation, photolysis, sorption to the glass container, or some other process is not known. However, the good agreement between the three methods, together with the observation that the results show no dependence on the level of recovery, leads us to assert that the losses affect the analyte and I.S. equally and therefore do not bias the results.

A typical chromatogram obtained by the LCUV method with I.S. is shown in Fig. 2. Other types of $\mathrm{C}_{18}$ columns have been tried and found to be usable when the methanol concentration in the mobile phase was adjusted to give similar retention times. Chromatograms obtained by the LC-MS method are very clean. typically showing only peaks from the analyte and I.S.

The limits of detection for each method were calculated from observations of the range of baseline noise, compared with the sensitivity. This has been found empirically to be equivalent to several other criteria [3]. Method detection limit for the LC-UV method was observed to be approximately $0.5-1.0 \mathrm{ng} / \mathrm{ml}$, and for the LCMS method $0.1-0.2 \mathrm{ng} / \mathrm{ml}$. The latter can be inferred from Fig. 3, which shows an LC-MS peak from the injection of a serum extract containing about 1 ng of Oltipraz. The detection limit for the on-line extraction method is, on one hand, degraded by the smaller sample size, and, on the other hand, enhanced by the elimination of analyte losses during evaporative concentration. The resulting method detection limit is about $3.0 \mathrm{ng} /$ $\mathrm{ml}$.

Comparative data from all three methods are shown in Table I. Two samples from each lot of spiked serum were run in duplicate by each method. The coefficients of variation (C.V.) are typical of those observed in our analyses of many other serum samples. The agreement between the three methods is also good.

The methods utilizing internal standards have been in use in our laboratory for two years to assign Oltipraz concentrations to serum samples

\section{TABLE I}

COMPARISON OF OLTIPRAZ CONCENTRATIONS DETERMINED BY THREE METHODS

\begin{tabular}{|c|c|c|c|c|c|c|}
\hline \multirow{2}{*}{$\begin{array}{l}\text { Serum } \\
\text { No. }\end{array}$} & \multicolumn{2}{|l|}{ LC-MS, I.S. } & \multicolumn{2}{|l|}{ LC-UV. I.S. } & \multicolumn{2}{|c|}{ LC-UV, external standard } \\
\hline & $\begin{array}{l}\text { Concentration } \\
(\mathrm{ng} / \mathrm{ml})\end{array}$ & $\begin{array}{l}\text { C.V. } \\
(\%)\end{array}$ & $\begin{array}{l}\text { Concentration } \\
(\mathrm{ng} / \mathrm{ml})\end{array}$ & $\begin{array}{l}\text { C.V. } \\
(\%)\end{array}$ & $\begin{array}{l}\text { Concentration } \\
(\mathrm{ng} / \mathrm{ml})\end{array}$ & $\begin{array}{l}\text { C.V. } \\
(\%)\end{array}$ \\
\hline 151 & 378 & 4.8 & 423 & 3.7 & 395 & 6.2 \\
\hline 152 & 210 & 6.2 & 203 & 3.8 & 203 & 4.8 \\
\hline 154 & 91.8 & 7.1 & 99.5 & 2.2 & 95.7 & 3.8 \\
\hline
\end{tabular}


circulated in performance evaluation exercises. The median of the absolute values of relative deviations in the results reported by other laboratories using similar and different methods is about $20 \%$.

The on-line extraction method was developed to provide a means of Oltipraz determination where specially synthesized internal standards are not available. Its lesser sensitivity could presumably be enhanced by extracting a larger volume of sample. It will not prove as well suited to routine use in clinical laboratories, however, unless the number of analyses is high enough to warrant setting up a chromatographic system with automated valving, dedicated to this analysis.

\section{ACKNOWLEDGEMENT}

We would like to acknowledge partial financial support for this research from the Division of Cancer Prevention and Control of the National Cancer Institute (USA) under interagency agreement Y01-CN-80655.

\section{REFERENCES}

1 C. W. Boone, G. J. Kelloff and W. E. Malone. Cancer Res., 50 (1990) 2.

2 J. L. Bennett, J. McMillan and N. V. Dimitrov, J. Chromalogr., 573 (1992) 146.

3 R. G. Christensen and W. E. May, J. Liquid Chromatogr., 1 (1978) 385. 


\section{ASCORBIC AND DEHYDROASCORBIC ACIDS ANALYSES}

This section contains two papers pertaining to the analysis of ascorbic acid and dehydroascorbic acid in human plasma. It describes the preparation and stability of quality control materials and calibration standards. Two HPLC methods for analysis are described in detail as well as procedures for optimum sample handling and analyte extraction. The points listed below are some of the more important factors related to this analysis.

\section{Important Factors}

\section{Calibration and Sample Preparation}

- All plasma samples must be preserved immediately after the cells are removed, preferably in metaphosphoric acid (MPA) at a final concentration of $5 \% \mathrm{w} / \mathrm{v}$.

- $\quad$ Plasma controls may be prepared from ascorbic-acid-free plasma by first adding dithiothreitol (DTT $1 \mathrm{mg} / \mathrm{mL}$ ) and then by adjusting the ascorbic acid content to the desired concentration by adding a solution of ascorbic acid in water:acetonitrile $(1: 3 \mathrm{v} / \mathrm{v})$ containing DTT $(1 \mathrm{mg} / \mathrm{mL})$. The samples may then be distributed into vials and lyophilized. If ascorbic acid is added to plasma without the dithiothreitol, it will be rapidly oxidized.

- Frozen plasma samples should be defrosted in a $20{ }^{\circ} \mathrm{C}$ water bath for no more than ten min. A longer duration or a higher temperature will lead to some ascorbic acid oxidation.

- Samples preserved in MPA should be mixed thoroughly before sampling in order to evenly suspend the precipitate.

- Sampling should be done with a diluting pump so that a plasma sample is washed out of the pipette with at least five volumes of distilled water or some other non-precipitating solvent. The viscosity of the plasma does not permit full delivery of the sample without a diluting or washout step.

- The addition of acetonitrile achieves a more complete precipitation of the proteins, prevents the accumulation of insoluble material at the top of the column, and extends the column life. 
- The assay is performed using calibration standards prepared with dithiothreitol $(1 \mathrm{mg} / \mathrm{mL})$ in acetonitrile and water $3: 1 \mathrm{v} / \mathrm{v}$. These standards are stable for 1 week when stored at $4{ }^{\circ} \mathrm{C}$. Each analysis run contains duplicate standards at each concentration, (i.e., six vials, two at each concentration). Two vials are analyzed at the beginning, the middle, and at the end of the assay to ensure that the conditions are constant.

\section{Chromatography}

- The chromatography was performed on a "Fast Acid" analysis column, $100 \times 7.8 \mathrm{~mm}$.

- The separation was isocratic using a solvent composed of $0.1 \mathrm{~mol} / \mathrm{L}$ formic acid and $5 \%$ acetonitrile in water at a flow rate of $1 \mathrm{~mL} / \mathrm{min}$. The column temperature was $30{ }^{\circ} \mathrm{C}$. Detection was electrochemical at +0.7 volts.

For further information, please contact Sam Margolis at (301)975-3137. 


\title{
Ascorbic and Dehydroascorbic Acids Measured in Plasma Preserved with Dithiothreitol or Metaphosphoric Acid
}

\author{
Sam A. Margolis, ${ }^{1}$ Robert C. Paule, ${ }^{1}$ and Regina G. Ziegier ${ }^{2}$
}

We describe a rapid method for accurately and precisely measuring ascorbic acid and dehydroascorbic acid in plasma. Total analysis time is $<10 \mathrm{~min}$, replicate analyses of a single pool provide precisions $\leq 2 \%$, and values measured in supplemented samples agree with known concentrations of 4.68 and $11.83 \mathrm{mg} / \mathrm{L}$. The stability and homogeneity of lyophilized plasma samples supplemented with ascorbic acid and dithiothreitol are documented. We also describe a procedure in which metaphosphoric acid (50 g/L) is used to prepare a reference material for the measurement of ascorbic acid and dehydroascorbic acid. The procedure for both acids consists of first measuring the native ascorbic acid, then reducing the dehydroascorbic acid, at neutral $\mathrm{pH}$, with dithiothreitol, and finally measuring the total ascorbic acid; dehydroascorbic acid is then determined by difference. The metaphosphoric-acid-treated samples were stable at $-70^{\circ} \mathrm{C}$, but stability decreased with temperature over the range examined, $4-50^{\circ} \mathrm{C}$.

Additional Keyphrases: reference materials - analytical precautions

Ascorbic acid (AA) and dehydroascorbic acid (DHAA) are normally present in both human and animal tissues and plasma. ${ }^{3}$ Both the chemical and biochemical destruction of AA occurs solely through the pathway of AA to DHAA to diketogulonic acid-the first reaction being reversible, the second irreversible (1). Accurate measurement of $\mathrm{AA}$ and DHAA in biological samples such as plasma depends on a reliable method for stabilizing these materials in the matrix of interest and the availability of a reliable assay for each analyte. Such measurements are necessary to the design and interpretation of epidemiological studies defining the factors affecting the distribution of $\mathrm{AA}$ and DHAA in blood and other tissues.

Currently there are three general procedures for quantifying the sum of both analytes (total AA): $(a)$ the dinitrophenylhydrazine method (2), (b) the o-phenylenediamine method (3-6), and (c) the reduction of DHAA by sulfhydryl reagents $(7-13)$.

The first two procedures, both based on the production of a derivative of DHAA with either dinitrophenylhydrazine or o-phenylenediamine, are used to measure the DHAA content and, indirectly, to measure total AA after the

\footnotetext{
${ }^{1}$ Organic Analytical Chemistry Division, Center for Analytical Chemistry, National Measurement Laboratory, National Institute for Standards and Technology, Gaithersburg, MD 20899.

${ }^{2}$ Environmental Epidemiology Branch, Epidemiology and Biostatistics Program, Division of Cancer Etiology, National Cancer Institute, Bethesda, MD 20892.

${ }^{3}$ Nonstandard abbreviations: AA, ascorbic acid; DHAA, dehy. droascorbic acid; MPA, metaphosphoric acid; HPLC, high-pressure liquid chromatography; DTT, dithiothreitol; and EC, electrochemical.
}

Received April 12, 1990; accepted July 27, 1990. native AA has been oxidized to DHAA (1). However, both of these reagents react with other reductones, including diketogulonic acid, and this can lead to measurement errors in a simple chromogenic assay (1).

The last two procedures have been used in conjunction with chromatographic methods. Method $b$ has been used for both pre- and postcolumn derivatization of DHAA with $o$-phenylenediamine and method $c$ for precolumn reduction of DHAA. The advantage of method $b$ is that it measures DHAA directly. These last two procedures involve either complex extractions that require the use of internal standards (4), an insensitive ultraviolet method for AA detection $(5,13)$, or a lengthy separation procedure $(6)$.

The procedure we used in this study (a variant of procedure c) involves sulfhydryl reagents $(7-13)$ to reduce DHAA to AA, and requires little additional sample manipulation. Diketogulonic acid is unaffected by dithiothreitol (DTT). This procedure measures only AA directly; DHAA is measured indirectly after its reduction to AA. The complete assay can be done by HPLC in $8 \mathrm{~min}$ with use of a very sensitive electrochemical (EC) detector at a constant electrode potential. To measure DHAA, one must analyze each sample twice, once for native AA concentration and once after the addition of DTT, to measure total AA content. The DHAA content is calculated as the difference between the values for native AA and total AA.

Accurate results entail both accurate analyses and stable samples. However, there is a paucity of published data on the long-term stability of AA in acid-stabilized serum or plasma $(3-5,14,15)$. In one report (5) it was demonstrated that $\mathrm{AA}$ and DHAA were stable when stored at $-20^{\circ} \mathrm{C}$ for 30 days in $100 \mathrm{~mL} / \mathrm{L}$ metaphosphoric acid (MPA). In a second (15), AA was found to be stable for five weeks at $-70{ }^{\circ} \mathrm{C}$ in $50 \mathrm{~mL} / \mathrm{L}$ MPA. In two studies $(3,14)$ total AA was measured in plasma. Bradley et al. (14) demonstrated that AA was stable in plasma for 21 days at $4,-20$, and $-70{ }^{\circ} \mathrm{C}$, in the presence of either MPA or trichloroacetic acid; Baker et al. (3) found AA to be stable for seven days at $-70{ }^{\circ} \mathrm{C}$ in EDTA-perchloric acid. Using a colorimetric assay, they $(3,14)$ observed an initial, unexplained $5 \%$ to $13 \%$ increase in the concentration of AA within the first week of storage, and Baker et al. (3) noted that their colorimetric results did not agree with results they obtained with an HPLC-EC method. Nevertheless, the concentration of AA determined by the HPLC method corresponded to the amount added to AA-free plasma, suggesting a systematic bias in the determination of AA by the dinitrophenylhydrazine method. We have reported that AA in plasma can be preserved by adding DTT and have demonstrated that all of the added AA was accounted for after 88 weeks in samples stored at $-70^{\circ} \mathrm{C}(13)$.

The objectives of the present study were $(a)$ to demonstrate the accuracy and precision (including some of the sources of systematic error) of the measurements determined by a modified version of our recently reported HPLC-EC method (13); (b) to demonstrate the feasibility of preparing a plasma pool suitable for use as a reference 
material in field studies; (c) to show that DHAA formed by the oxidation of AA in plasma at $22^{\circ} \mathrm{C}$ can be quantitatively converted to $\mathrm{AA}$; and $(d)$ to demonstrate that no measurable degradation of AA occurs in MPA-treated plasma at $-70^{\circ} \mathrm{C}$, but at 4,25 , and $50^{\circ} \mathrm{C}$ AA is more or less slowly oxidized to DHAA and other products.

\section{Materials and Methods ${ }^{4}$}

\section{Materials}

AA and DTT were purchased from Sigma Chemical Co., St. Louis, MO; metaphosphoric acid from Fisher Scientific, Fair Lawn, NJ; and the plasma from Western States Plasma Corp., Fallbrook, CA. The acetonitrile was "HPLC" grade. The AA, examined by proton magnetic resonance, contained $<0.1 \%$ protonated impurities, including DHAA and its degradation products. The processed plasma was stored at $-20{ }^{\circ} \mathrm{C}$ until used.

The method of AA analysis is a modification of that described in reference 13 as HPLC Method 1. A $100 \times 7.8$ $\mathrm{mm}$ "fast acid analysis" column (Bio-Rad Laboratories, Richmond, CA) was used at a flow rate of $1 \mathrm{~mL} / \mathrm{min}$ and the EC detector was a Model 400 (EG\&G, Princeton Applied Research, Princeton, NJ). Analysis time was shortened from $35 \mathrm{~min}$ to $8 \mathrm{~min}$. A series of three calibration standards (three AA concentrations that bracketed the sample concentrations) were run at the beginning and end of each day, to assess the stability of the detector and to provide a calibration curve for use in calculating the AA concentration of the samples (13). All serum samples were aliquoted with a calibrated automated positive-displacement pipet in either the dispensing (for reference-material preparation) or diluting (for sample analysis) mode.

\section{Preparation of AA-Supplemented Plasma Samples}

Set 1. Quality-control sample set. We prepared three 160-sample groups of lyophilized plasma: $(a)$ a group of unsupplemented plasma samples, (b) a group supplemented to give a low-normal concentration of $\mathrm{AA}$, and (c) a group supplemented to give a high-normal concentration of AA. DTT was added to plasma at room temperature to give a concentration of $1 \mathrm{~g} / \mathrm{L}$. This plasma (relative density 1.025) was divided into three lots, two of which were supplemented by adding a weighed amount of an AA solution (1.184 g of AA per gram of $10 \mathrm{~g} / \mathrm{L}$ aqueous DTT:acetonitrile, 1:3 by vol), such that the final AA concentration of each lot of reference material was 4.68 and $11.83 \mathrm{mg}$ per liter of plasma for the low- and high-normal samples, respectively. Each lot of reference material was well mixed, after which 1-mL samples were aliquoted, lyophilized, and stored at $-70^{\circ} \mathrm{C}$ as previously described (13).

Each sample was prepared for analysis by equilibrating it at room temperature and adding $0.97 \mathrm{~mL}$ of distilled water. After the solids were completely dissolved, $0.1 \mathrm{~mL}$ of reconstituted plasma was diluted with $1 \mathrm{~mL}$ of distilled water into a vial containing $160 \mu \mathrm{L}$ of a $400 \mathrm{~g} / \mathrm{L}$ solution of metaphosphoric acid; $400 \mu \mathrm{L}$ of acetonitrile was added, and the vial was capped and vortex-mixed for $15 \mathrm{~s}$. The result-

\footnotetext{
${ }^{4}$ Identification of any commercial product does not imply recommendation or endorsement by the National Institute of Standards and Technology, nor does is imply that the material or equipment identified is necessarily the best available for the purpose.
}

ing suspension was centrifuged $\left(1000 \times g, 30 \mathrm{~min}, 4^{\circ} \mathrm{C}\right)$ and the supernatant fluid was transferred to $1.8-\mathrm{mL}$ autosampler vials and analyzed by HPLC.

Set 2. Samples preserved with metaphosphoric acid. The frozen human plasma was thawed and three 100-sample lots were prepared: $(a)$ a group of unsupplemented plasma samples, (b) a group supplemented with a low-normal concentration of $\mathrm{AA}$, and $(c)$ a group supplemented with a high-normal concentration of AA. Two lots of plasma were supplemented by adding a weighed amount of an AA solution (49.56 mg of AA per $53.24 \mathrm{~g}$ of aqueous metaphosphoric acid, $100 \mathrm{~g} / \mathrm{L}$ ) such that the final concentration of AA in each lot was 4.87 and $11.75 \mathrm{mg}$ per liter of plasma (low and high normal, respectively). Each plasma lot was thoroughly mixed. The solutions were exposed to $22^{\circ} \mathrm{C}$ for $2 \mathrm{~h}$, permitting partial oxidation of AA to DHAA. Therefore, the $b$ and $c$ samples could be used to analyze for both $\mathrm{AA}$ and DHAA, and they mimicked real samples. Of these plasma samples, $0.5-\mathrm{mL}$ aliquots were then mixed with 0.5 $\mathrm{mL}$ of a $100 \mathrm{~g} / \mathrm{L}$ solution of metaphosphoric acid, in glass ampules, which were then sealed, their contents frozen, and stored at $-70^{\circ} \mathrm{C}$.

At the time of analysis, the test-plasma samples preserved with metaphosphoric acid were thawed in a water bath at $20^{\circ} \mathrm{C}$; thawing was complete within $10 \mathrm{~min}$. Two analyses were performed on each sample, the first to measure the native AA content and the second to measure the total AA (i.e., AA + DHAA) after reduction with DTT.

Samples for the first analysis were prepared by diluting $0.200 \mathrm{~mL}$ of sample with $1 \mathrm{~mL}$ of distilled water in a vial containing $160 \mu \mathrm{L}$ of metaphosphoric acid $(400 \mathrm{~g} / \mathrm{L}) ; 0.400$ $\mathrm{mL}$ of acetonitrile was added, the vial was capped, and the sample was vortex-mixed for $15 \mathrm{~s}$. After centrifuging the resulting suspension $\left(1000 \times \mathrm{g}, 30 \mathrm{~min}, 4^{\circ} \mathrm{C}\right)$, we transferred the supernatant fluid to $1.8-\mathrm{mL}$ autosampler vials and analyzed it by HPLC.

The samples for the second analysis were prepared in the same manner as for the first analysis except that $200 \mu \mathrm{L}$ of test plasma sample was diluted with $1 \mathrm{~mL}$ of distilled water in a vial containing $200 \mu \mathrm{L}$ of a solution of $0.5 \mathrm{~mol}$ of $\mathrm{K}_{2} \mathrm{HPO}_{4}$ and $1 \mathrm{~g}$ of DTT per liter. After $1 \mathrm{~h}$ at room temperature, $160 \mu \mathrm{L}$ of a $400 \mathrm{~g} / \mathrm{L}$ MPA solution and $400 \mu \mathrm{L}$ of acetonitrile were added, and samples were vortex-mixed and centrifuged as above. The supernatant fluid was then transferred to autosampler vials for analysis. All HPLC-EC measurements were corrected by calibration factors.

Statistical analysis. Measurement data on the samples from set 1 were examined. Separate analyses of variance were made for the low- and the high-concentration plasma samples. The organization of the data was that of a fourlevel hierarchical design. Analyses were made over 12 different days, with two different samples for each day, two aliquots taken from each sample, and two measurements made on each aliquot. Thus, for each concentration, 96 measurement results were used and four sources of variance were evaluated: days, samples, aliquots, and replicate measurements.

\section{Results}

The chromatograms of the unsupplemented plasma (Figure $1, A$ and $B$ ) indicate that it contained no electrochemically active material with a retention time at or near that of AA. Furthermore, treatment of this plasma with DTT did not result in the generation of any material that 


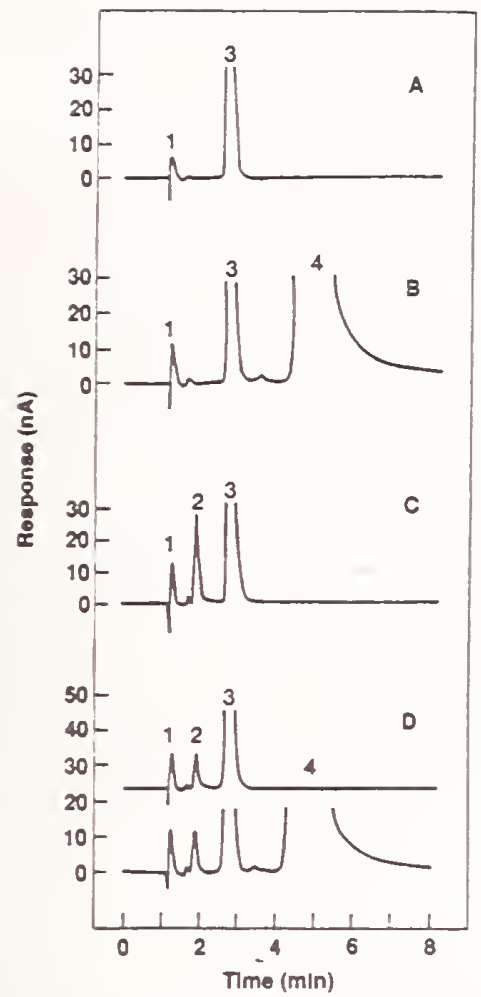

Fig. 1. Chromatograms of lyophilized and MPA-preserved human plasma and AA-supplemented plasma with and without DTT treatment

A. lyophilized plasma; $B$, plasma preserved in MPA $(50 \mathrm{~g} / \mathrm{L})$ and treated with dipotassium phosphate $(0.05 \mathrm{~mol} / \mathrm{L})$ and DTT (1 g/t) for $1 \mathrm{~h}$; $C$, lyophilized plasma containing DTT $(1 \mathrm{~g} / \mathrm{L})$ and supplemented with $11.83 \mathrm{mg}$ of AA per liter: D. plasma preserved with MPA $(50 \mathrm{~g} / \mathrm{L})$ and supplemented with $A A, 11.75$ $\mathrm{mg} / \mathrm{L}$, upper trace, no DTT, lower trace, treated with DTT $(1 \mathrm{~g} / \mathrm{L})$ and dipotassium phosphate (0.05 mol/L) for $1 \mathrm{~h}$. Peak 1, MPA; 2, AA; 3, uric acid; 4. DTT

co-chromatographed with AA. Thus the unsupplemented plasma matrix contained neither AA nor DHAA (which was reduced to AA with DTT), and all of the material measured in all samples represented either added AA or material converted to AA.

\section{Measurement of AA in Lyophilized Plasma Stored at $-70^{\circ} \mathrm{C}$ (Set 1)}

The concentration of AA in the two supplemented lots of plasma (AA, 4.68 and $11.83 \mathrm{mg} / \mathrm{L}$ ) was measured in duplicate on each of 12 days over a 21-day period. Twenty-four samples were selected without conscious bias from each lot and two were analyzed on each day. Analysis of variance of these data (Table 1) gives the various sources of imprecision contributing to the total variability of a single measurement. The total imprecision (i.e., the CV) of a single measurement for each lot of samples was $2 \%$ (11.76 \pm 0.26 and $4.70 \pm 0.11 \mathrm{mg} / \mathrm{L}$ ). This uncertainty is small and the measured values vary from the gravimetric value by $<1$ SD (Table 1). These results demonstrate the stability and homogeneity of these reference materials relative to the day of analysis and the order of sample preparation.

\section{Measurement of AA and DHAA in Frozen Plasma Preserved with MPA (Set 2)}

The following studies were done to examine the accuracy and precision of the measurement of existing AA, total AA after reduction of the DHAA in the serum sample with
Table 1. Summary of Analysis of Variance of Plasma Samples, Set 1, Supplemented with AA at High- and Low-Normal Concentrations

\begin{tabular}{llc} 
& \multicolumn{2}{c}{ Ascorbic acld, $\mathrm{mg} / \mathrm{L}$} \\
AA added & 11.83 & 4.68 \\
Grand mean of all 96 & 11.76 & 4.70 \\
$\quad$ measurements & & \\
Source of variability & Component of SD, $\mathrm{mg} / \mathrm{L}$ \\
Day & $\mathrm{nS}^{\mathrm{a}}$ & 0.07 \\
Sample & 0.23 & $\mathrm{~ns}^{\mathrm{a}}$ \\
Aliquot & 0.07 & 0.06 \\
Replicate measurement & 0.10 & 0.06 \\
SD of a single & 0.26 & 0.11 \\
$\quad$ measurement & & \\
CV, \% & $\sim 2$ & -2 \\
${ }^{a}$ ns $=$ not significant. & & \\
\hline
\end{tabular}

DTT, and DHAA by difference (total AA - existing AA). Because the reduction of DHAA by DTT is $\mathrm{pH}$ dependent (7), we adjusted the $\mathrm{pH}$ of each sample with dipotassium phosphate. The precipitated material completely dissolved and the DHAA was completely reduced to AA within 10 min at room temperature $\left(22^{\circ} \mathrm{C}\right)$. The resulting AA concentration remained constant for at least $90 \mathrm{~min}$ after the sample was prepared. In the absence of the dipotassium phosphate the reduction of the DHAA was still incomplete after the mixture had stood for an hour at room temperature.

Before being preserved with MPA, the initial decrease in AA (11.75 and $4.87 \mathrm{mg} / \mathrm{L})$ in plasma at $22^{\circ} \mathrm{C}$ for $2 \mathrm{~h}$ was 1.60 and $0.98 \mathrm{mg} / \mathrm{L}$, respectively (Table 2). After being preserved with MPA, these samples were analyzed for DHAA content and for analytical recovery of supplemented AA (i.e., AA + DHAA content) over a period of 98 days. The total AA remained constant in MPA $(50 \mathrm{~g} / \mathrm{L})$ when the sample was stored at $-70^{\circ} \mathrm{C}$. These results indicate that the oxidized AA, namely DHAA, could be quantitatively converted to AA by DTT, and that no diketogulonic acid was formed. This is demonstrated by the fact that the values obtained for AA after DTT treatment (11.69 and $5.03 \mathrm{mg} / \mathrm{L})$ were not statistically different $(P>0.05$, Student's $t$-test) from the amount gravimetrically added to the plasma at the beginning of the experiment (11.75 and 4.87 $\mathrm{mg} / \mathrm{L}$, Table 2). These results demonstrate the relative homogeneity of the samples preserved with MPA and indicate that the sample-to-sample variation in total AA

\section{Table 2. Summary of Analyses of AA-Supplemented Plasma Samples, Set 2, Preserved In MPA, $50 \mathrm{~g} / \mathrm{L}$} Concentration, $\mathrm{mg} / \mathrm{L}$

\section{AA added}

(No. of samples)

$\mathrm{AA}^{a}$

Total $A A^{\circ}$

DHAA $^{\circ}$
11.75

(10)

$10.09 \pm 0.04$

$11.69 \pm 0.06$

$1.60 \pm 0.07$
4.87

$4.05 \pm 0.13$

$5.03 \pm 0.06^{c}$

$0.98 \pm 0.14$ a Mean \pm SD of the mean.

- Total ascorbic acid (mean \pm SD of mean) was determined as described in the text with use of DTT as a reducing agent.

${ }^{c}$ This value does not differ significantly $(P>0.05$ by Student's $\neq$ test) from the gravimetric value.

${ }^{\circ}$ Determined by subtracting the mean $A A$ value from the mean total $A A$ value. 
content is similar to that observed for those samples prepared in DTT and then lyophilized and stored at $-70^{\circ} \mathrm{C}$.

The $\mathrm{CV}$ for the measurement of $\mathrm{AA}$ in both sample lots before the reduction of DHAA with DTT, $0.4 \%$ and $3 \%$ $(10.09 \pm 0.04$ and $4.05 \pm 0.13 \mathrm{mg} / \mathrm{L})$, is similar to the $\mathrm{CV}$ for the total AA value, $0.5 \%$ and $1 \%$ (11.69 \pm 0.06 and 5.03 $\pm 0.06 \mathrm{mg} / \mathrm{L}$, Table 2$)$. The DHAA content (1.60 \pm 0.07 and $0.98 \pm 0.14 \mathrm{mg} / \mathrm{L}$ )-i.e., the "total AA" minus "AA"-can be determined to the same general precision as the "total AA" and "AA." The results indicate that $<1 \mathrm{mg}$ of DHAA per liter can be precisely measured.

Stability of AA in Plasma Stabilized with MPA (Set 2) at 4, 25 , and $50^{\circ} \mathrm{C}$

In preliminary studies we thawed our samples by putting them in "warm water" for a variable period of time. Under these uncontrolled conditions we observed measurable variation in the initial AA content. Thus at increased temperatures, plasma AA preserved with MPA does not appear to be stable. We have now examined this instability at 4,25 , and $50^{\circ} \mathrm{C}$, using the plasma samples from set 2 . At $4{ }^{\circ} \mathrm{C}$ AA degraded slowly. The degradation was detectable in the high-normal plasma samples after only one day, and it continued for 14 days (Table 3 , experiments 1 and 2). In the low-normal plasma samples (Table 3, experiment 3 ) about $80 \%$ of the AA was lost after 14 days. More importantly, the total AA content was reduced $42 \%$. This indicates that some of the DHAA was also degraded along with the AA. At $25^{\circ} \mathrm{C}$, detectable oxidation of AA occurred within $5 \mathrm{~h}$, and after $24 \mathrm{~h} 18 \%$ of the AA had been oxidized

Table 3. Stability of Ascorbic and Dehydroascorbic Acids in MPA, $50 \mathrm{~g} / \mathrm{L}$, at $4{ }^{\circ} \mathrm{C}$

Day AA Total AA ${ }^{\circ}$ DHAAC

Experiment 1

$\begin{array}{llll}0 & 9.9 \pm 0.22 & 11.6 \pm 0.08 & 1.7 \\ 1 & 9.2 \pm 0.19 & 11.6 \pm 0.15 & 2.4 \\ 2 & 9.1 \pm 0.10 & 11.0 \pm 0.12 & 1.9 \\ 4 & 8.6 \pm 0.12 & 11.2 \pm 0.06 & 2.6 \\ 7 & 8.5 \pm 0.09 & 10.6 \pm 0.11 & 2.1 \\ 9 & 7.6 \pm 0.06 & 9.9 \pm 0.03 & 2.3 \\ \text { loss } & 2.3 & 1.7 & \end{array}$

Total loss

2.3

1.7

$\begin{array}{cr}\text { Experiment } 2 & \\ 0 & 10.1 \pm 0.14 \\ 3 & 8.8 \pm 0.14 \\ 7 & 8.7 \pm 0.08 \\ 10 & 7.9 \pm 0.13 \\ 14 & 7.2 \pm 0.10\end{array}$

Total loss

2.9

$$
\begin{array}{r}
11.6 \pm 0.06 \\
10.9 \pm 0.12 \\
11.2 \pm 0.05 \\
10.1 \pm 0.05 \\
9.7 \pm 0.12
\end{array}
$$

1.9

1.5

2.1

2.5

2.2

2.5

\section{Experiment 3}

$\begin{array}{cl}0 & 3.9 \pm 0.18 \\ 3 & 3.3 \pm 0.10 \\ 7 & 2.9 \pm 0.07 \\ 10 & 1.9 \pm 0.10 \\ 14 & 0.7 \pm 0.06 \\ \text { loss } & 3.2\end{array}$

$$
\begin{aligned}
& 5.0 \pm 0.06 \\
& 4.6 \pm 0.02 \\
& 4.4 \pm 0.04 \\
& 3.6 \pm 0.07 \\
& 2.9 \pm 0.09 \\
& 2.1
\end{aligned}
$$

${ }^{a}$ Each number represents the mean (and SD) of four measurements.

${ }^{b}$ Ascorbic acid content measured after treatment of the sample with DTT.

$c$ The difference between the AA value and the total AA value.
(Table 4). At $50^{\circ} \mathrm{C}$ the degradation of AA was even faster, $16 \%$ in $3 \mathrm{~h}$ (Table 4 ).

\section{Discussion}

Validity of AA and DHAA measurement in clinical plasma samples depends on the availability of a method of demonstrated precision and accuracy for measurement of each analyte. The modified method described here is characterized by increased speed of analysis ( $8 \mathrm{~min}$ ), baseline peak resolution comparable with that of our previous method (13), and the elimination of baseline drift. Figure 1 clearly demonstrates the baseline resolution of all peaks. Furthermore, the chromatograms of the unsupplemented plasma to which DTT was added indicate that there was no AA, DHAA, or any "contaminating" peaks. However, for patients receiving special therapy, it may be necessary to demonstrate that no interfering materials are present by measuring the AA content in the presence and absence of ascorbate oxidase (EC 1.10.3.3). 'The baseline drift observed in earlier experiments (13) was reduced by use of the EC detector such that negligible signal degeneration was observed during an 8-h period.

A major source of measurement error is imprecision in sampling the plasma. This is a function of the viscosity of the plasma. We have minimized this error by using an automated diluting pipet. With this iristrument, an accu-

\begin{tabular}{|c|c|c|c|}
\hline \multicolumn{4}{|c|}{$\begin{array}{l}\text { Table 4. Stability of Ascorbic and Del } \\
\text { Acids in MPA, } 50 \mathrm{~g} / \mathrm{L}\end{array}$} \\
\hline & AA & Total $A A^{B}$ & DHAAC \\
\hline Milnutes" & \multicolumn{3}{|c|}{$\mathrm{mg} / \mathrm{L}$} \\
\hline \multicolumn{4}{|c|}{ Temperature $25^{\circ} \mathrm{C}$} \\
\hline 15 & $10.3^{d}$ & $12.0^{\circ}$ & 1.7 \\
\hline 60 & 10.1 & 12.0 & 1.9 \\
\hline 120 & 10.2 & 11.8 & 1.6 \\
\hline 180 & 9.8 & 11.8 & 2.0 \\
\hline 300 & 9.7 & 11.6 & 1.9 \\
\hline 1440 & 8.4 & 11.0 & 2.6 \\
\hline Total loss & 1.9 & 1.0 & \\
\hline \multicolumn{4}{|c|}{ Temperature $50^{\circ} \mathrm{C}$} \\
\hline 0 & 10.1 & 11.6 & 1.5 \\
\hline 20 & 9.8 & 11.7 & 1.9 \\
\hline 40 & 9.8 & 11.8 & 2.0 \\
\hline 60 & 9.4 & 11.8 & 2.4 \\
\hline 120 & 8.9 & 11.2 & 2.3 \\
\hline 180 & 8.5 & 10.7 & 2.2 \\
\hline Total loss & 1.6 & 0.9 & \\
\hline \multicolumn{4}{|c|}{$\begin{array}{l}\text { a Elapsed time after the sample was transferred from }-70^{\circ} \mathrm{C} \text { directly into } \\
\text { the controlled-temperature bath. } \\
{ }^{b} \text { Ascorbic acid content measured after treatment of the sample with DTT. } \\
{ }^{C} \text { The difference between the AA value and the total AA value. } \\
{ }^{d} \text { Each value represents the mean of two determinations. }\end{array}$} \\
\hline
\end{tabular}
rate aliquot of plasma is taken and then the sampling pipet is purged with at least five volumes of diluent to wash out the viscous plasma. We tested the dependability of this method by using a single lot of stable reference plasma samples that had been supplemented with $\mathrm{AA}$ and preserved as previously described (13). The results of such an experiment (Table 1) indicate that this method accurately measures the supplemented concentrations of AA, i.e., measured values of $11.76 \pm 0.26$ and $4.70 \pm 0.11 \mathrm{mg} / \mathrm{L}$ as compared with the respective gravimetric values of 11.83 
and $4.68 \mathrm{mg} / \mathrm{L}$. The precision of both the low- and highconcentration AA measurements is $\sim 2 \%$. The error contributions of the different components of the analytical method were assessed by analysis of variance. These components (Table 1 ) included $(a)$ the variability due to the handling and processing of samples on different days; (b) the between-sample variability for a given analyte concentration; (c) the variability in the sample preparation procedure, including aliquoting the sample, adding reagents, and precipitating the proteins; and (d) variability between duplicate analyses on the same aliquot that is attributable to the variation in instrument settings. From a practical viewpoint, all four sources of variability are small. The difference between the gravimetric measurement and the observed values (an index of the accuracy of the measurement) falls within the precision of the measurement, the CV being about $2 \%$ of the measured value for a single measurement. This supports the conclusion that there is no outstanding source of systematic bias.

Furthermore these results demonstrate an acceptable level of stability and homogeneity for both sets of samples (sets 1 and 2) and clearly indicate that our measurements are reproducible for both sets of samples. These results are consistent with our previous observation (13) on the stability of samples that were similar to set 1 . Because these samples are stable, this produçt may be a suitable control material for AA measurement by methods used to resolve $\mathrm{AA}$ and DTT.

The suitability of a method for preserving AA in biological samples collected for epidemiological studies and the valid interpretation of data from completed epidemiological studies depend on demonstrating the stability of plasma AA preserved by that method. One approach to evaluating the stability of $\mathrm{AA}$ in plasma is to measure, specifically, $\mathrm{AA}$ and its immediate degradation product, DHAA, so that storage procedures can be effectively evaluated. This is particularly important because some clinical samples may contain significant concentrations of $\operatorname{DHAA}(5,6)$.

For us to evaluate the stability of AA and DHAA in plasma preserved with MPA, it was necessary to prepare a reference material in which the total $\mathrm{AA}(\mathrm{AA}+\mathrm{DHAA})$ was accurately measured. This was achieved by gravimetrically adding a known amount of AA to plasma, and permitting partial oxidation of the AA to DHAA by letting the supplemented plasma stand at room temperature for $2 \mathrm{~h}$. The DHAA produced this way seemed to us to most closely approach the conditions under which DHAA is generated when samples are processed in the field and preserved with MPA.

DHAA was measured after neutralizing the plasma sample and reducing the DHAA to AA with DTT. The reduction of the DHAA was complete within $10 \mathrm{~min}$ and no further change was observed during the following $80 \mathrm{~min}$. The nonsupplemented plasma pool contained neither AA nor DHAA (Figure 1). The results in Table 1 clearly demonstrate that all of the added AA was accounted for as $\mathrm{AA}$ or DHAA and that both $\mathrm{AA}$ and total AA (and by inference the DHAA) are stable at $-70^{\circ} \mathrm{C}$ when preserved in MPA $(50 \mathrm{~g} / \mathrm{L})$. The small standard deviation indicates that the means for both supplemented lots are precise and that all of the values from day 6 to day 98 fell within a narrow range. These results, i.e., recovery of all supplemented AA, also indicate that the use of DTT $(1 \mathrm{~g} / \mathrm{L})$ in the presence of approximately $0.05 \mathrm{~mol}$ of dipotassium phosphate per liter (final concentration) effectively reduces all of the oxidized AA to AA. Thus, this method provides a suitable technique for measuring the sum of $\mathrm{AA}$ plus DHAA in biological samples after they have been preserved with MPA (50 $\mathrm{g} / \mathrm{L})$.

The identification of the degraded AA as DHAA is based on its reductive conversion to a material chromatographically identical to AA. Because the unsupplemented plasma does not contain any material that can be converted to a material that is chromatographically identical to $\mathrm{AA}$ or is electrochemically reducible, the material that was detected by reduction with DTT had to arise from the supplemented AA. The observation that DHAA in plasma can be reduced to AA by DTT (13) also supports the identification of the degraded AA as DHAA.

The studies described above (as opposed to those done on serum samples collected and preserved in field studies) were carried out under ideal conditions, where the samples were rapidly aliquoted and quickly cooled to $-70^{\circ} \mathrm{C}$. At the time of assay they were carefully thawed and prepared for assay. Close attention must be paid to this procedure because of the temperature instability of AA and DHAA. A study of the stability of AA and DHAA at 4,25 , and $50^{\circ} \mathrm{C}$, in the supplemented plasma samples preserved in MPA, indicates that degradation of $\mathrm{AA}$ can be detected at these temperatures (Tables 3 and 4 ). At $4{ }^{\circ} \mathrm{C}$, degradation of AA can be detected between 24 and 45 , h. By 14 days, 29\% ( 2.9 $\mathrm{mg} / \mathrm{L}$ ) of the remaining $\mathrm{AA}$ at the high concentration and $82 \%(3.2 \mathrm{mg} / \mathrm{L})$ at the low concentration were oxidized. At $25^{\circ} \mathrm{C}$, similar results were observed within $24 \mathrm{~h}(1.9 \mathrm{mg} / \mathrm{L})$ and at $50^{\circ} \mathrm{C}$, within $3 \mathrm{~h}(1.6 \mathrm{mg} / \mathrm{L})$. Furthermore, at all three temperatures a measurable amount of the total AA was not accounted for at the end of the experiment; evidently some of the DHAA was further degraded. Thus, under field conditions, MPA-treated clinical samples can be stored for a day at $4{ }^{\circ} \mathrm{C}$ without significant loss of AA or of its initial degradation product (DHAA), but higher temperatures incur a risk of significant loss. Frozen samples should be thawed before analysis by warming the samples at $20^{\circ} \mathrm{C}$ for $10 \mathrm{~min}$ to ensure minimal degradation. Furthermore, when MPA-preserved samples are kept at room temperature for extended periods in the absence of DTT, the AA can be expected to undergo degradation. These samples will not yield accurate measurements of native AA.

Under the conditions described above, we did not observe AA concentrations significantly exceeding the amount to which the plasma was supplemented. This suggests that earlier results obtained with a colorimetric method are in error, the source of which remains undefined $(3,14)$. We were also able to account for all of the DHAA that we generated. This is in contrast to the lesser recovery reported by Baker et al. (3), a discrepancy that may be the result of incomplete conversion of the DHAA dimer to the monomer in the plasma samples supplemented with commercial DHAA. Finally, we have confirmed that aged plasma can slowly oxidize AA to DHAA at a rate similar to that reported by Baker et al. (3) and that the DHAA resulting from the oxidation of $\mathrm{AA}$ can be determined by the method described herein.

In conclusion we have described a technique that is suitable for use in the future evaluation of the stability of AA and DHAA in whole blood and for the elucidation of suitable conditions for conserving the physiological ratio of these compounds so that the epidemiological significance of 
the natural concentrations of AA and DHAA can be explored.

We thank Dr. Robert Schaffer for his assistance in the preparation of the ascorbic-acid-supplemented human plasma samples and Dr. Willie May for coordination of the NIST/NCI Program. This study was supported in part by National Cancer Institute contract no. Y01-CP9-0506.

\section{References}

1. Pelletier O. Vitamin C. L-Ascorbic acid and dehydro-L-ascorbic acid [Review]. In: Augustin J, Klein BP, eds. Methods of vitamin assay. New York: John Wiley and Sons, Inc., 1985:303-46.

2. Roe JH, Mills MB, Oesterling MJ, Damron CM. The determination of diketo-L-gulonic, dehydro-L-ascorbic acid and L-ascorbic acid in the same tissue extract by the 2,4-dinitrophenylhydrazine method. J Biol Chem 1948;174:201-8.

3. Baker JK, Kapeghian J, Verlangieri A. Determination of ascorbic acid and dehydroascorbic acid in blood plasma samples. J Liq Chromatogr 1983;6:1333-44.

4. Huang T, Kissinger PT. Simultaneous LC determination of ascorbic acid in foods and biological fluids. Curr Separations 1989;9:20-3.

5. Lunec J, Blake DR. The determination of dehydroascorbic acid and ascorbic acid in serum and synovial fluid of patients with rheumatoid arthritis (RA). Free Radical Res Commun 1985;1:319.

6. Vanderslice JT, Higgs DJ. Chromatographic separation of ascorbic acid, isoascorbic acid, dehydroascorbic acid and dehydroisoascorbic acid and their quantitation in food products. J Micronutr Anal 1988;4:109-18.

7. Okamura M. An improved method for determination of L- ascorbic acid and L-dehydroascorbic in blood plasma. Clin Chim Acta 1980;103:259-68.

8. Schreiber J, Lohmann W, Unverzagt D, Otter A. Determination of ascorbic acid, dehydroascorbic acid and isoascorbic acid in blood. Fresenius Z Anal Chem 1986;325:473-5.

9. Otsuka M, Kurata T, Arakawa N. The tissue distribution of L-ascorbic acid and dehydro-L-ascorbic acid in the guinea pigs injected intravenously with dehydro-L-ascorbic acid. J Nutr Sci Vitaminol (Tokyo) 1986;32:259-66.

10. Doner LW, Hicks KB. High performance liquid chromatographic separation of ascorbic acid, erythorbic acid, dehydroascorbic acid and diketogulonic acid. Anal Biochem 1981;115:225-30.

11. Dennison DB, Brawley TG, Hunter GLK. Rapid high-performance liquid chromatographic determination of ascorbic acid and combined ascorbic acid-dehydroascorbic acid in beverages. J Agric Food Chem 1981;29:927-9.

12. McGahan MC. Ascorbic acid levels in aqueous and vitreous humors of the rabbit: effects of inflammation and ceruloplasmin.

Exp Eye Res 1985;41:291-8.

13. Margolis SA, Davis TP. Stabilization of ascorbic acid in human plasma, and its liquid chromatographic measurement. Clin Chem 1988;34:2217-23.

14. Bradley DW, Emery G, Maynard JD. Vitamin C in plasma: a comparative study of the vitamin stabilized with trichloroacetic acid or metaphosphoric acid and the effects of storage at $-70^{\circ}$, $-20^{\circ}, 4^{\circ}$, and $25^{\circ}$ on the stabilized vitamin. Clin Chim Acta $1973 ; 44: 47-52$.

15. Kutnink MA, Skala JH, Sauberlich HE, Omaye ST. Simultaneous determination of ascorbic acid, isoascorbic acid (erythorbic acid), and uric acid in human plasma by high-performance liquid chromatography with amperometric detection. J Liq Chromatogr $1985 ; 8: 31-46$. 


\section{Stabilization of Ascorbic Acid in Human Plasma, and Its Liquid-Chromatographic Measurement}

\section{Sam A. Margolls' and Thomas P. Davis ${ }^{2}$}

We describe two independent HPLC procedures for the rapid, accurate analysis for ascorbic acid in human plasma. No sample extraction or phase separation is required. We also describe a procedure for prepaning a human plasma reference material for use in clinical laboratory analysis for ascorbic acid. The ascorbic acid in plasma can be determined in $15 \mathrm{~min}$, with as little as $50 \mu \mathrm{L}$ of sample. Analytical recoveries are near $100 \%$ with direct injection of deproteinized plasma. Extensive stability data under several conditions, with dithiothreitol as a preservative (antioxidant), indicate that ascorbic acid remains stable in stored plasma for as long as $\mathbf{5 7}$ weeks. CVs for round-robin analysis of 11 normal human blood samples by two independent methods were between $0.1 \%$ and $5.3 \%$. These clinical samples appear to be stable for at least 50 days under the described conditions of stabilization and sample treatment. Finally, because ascorbic acid prepared by the described procedures is stable at room temperature for at least $18 \mathrm{~h}$, these methods can be readily adapted to clinical laboratory automation at room temperature.

Additional Keyphrases: dithiothreitol preservative - reference materials - sample handling - electrochemical detection

Currently, the recommended method for preparing plasma for assay of ascorbic acid (AA) consists of drawing the blood into a suitable anticoagulant, transferring it to the laboratory, removing the cellular material by centrifuga. tion, and adding metaphosphoric acid (MPA) to precipitate the proteins and prevent oxidation of the AA. ${ }^{3}$ The resulting suspension can then be stored at $-80^{\circ} \mathrm{C}$ for extended periods (1-4) with no decrease in apparent AA content. However, in routine laboratory procedures, $15 \mathrm{~min}$ to several hours can elapse between blood sampling and treatment to stabilize AA, or between the thawing of a frozen specimen and its analysis. A significant loss of ascorbic acid content, and erroneous measurements of ascorbic acid in plasma, can result (6). We confirmed the utility of dithiothreitol (DTT) as a preservative for $\mathrm{AA}$ in solution (7), and demonstrated that dilute solutions of $\mathrm{AA}$ in acetonitrile:water ( $3: 1$ by vol) containing $1 \mathrm{~g}$ of DTT per liter are stable for 60 days when stored at $-20^{\circ} \mathrm{C}$ under argon in sealed glass ampules (3).

Our objective in this study was to evaluate the degree of stabilization of AA conferred by DTT at a final concentration of $1 \mathrm{~g} / \mathrm{L}$ (5.4 mmol/L) under the following conditions: (a) in plasma, when it is added at the same time that the plasma is supplemented with $\mathrm{AA}$; (b) in blood, when the blood is drawn into heparinized tubes containing a solution of DTT such that the final concentration of DTT is $1 \mathrm{~g} / \mathrm{L}$; and

\footnotetext{
1 Organic Analytical Chemistry Division, Center for Analytical Chemistry, National Bureau of Standards, Gaithersburg, MD 20899.

2 University of Arizona Health Sciences Center, Department of Pharmacology, College of Medicine, Tucson, AZ 85724.

${ }^{3}$ Nonstandard abbreviations: AA, ascorbic acid; DHAA, dehydroascorbic acid; MPA, metaphosphoric acid; DTT, dithiothreitol; NBS, National Bureau of Standards.

Received April 28, 1988; accepted July 7, 1988.
}

(c) in plasma, when it is added with MPA (17 g/L final concentration). In addition we report two simple, rapid, accurate, and precise HPLC methods for determining AA in plasma that can be easily adapted for routine use by clinical chemistry laboratories.

\section{Materlals and Methods4}

We used two independent HPLC analytical procedures. Method 1, performed at the National Bureau of Standards (NBS), was used to evaluate the stability of AA in DTTsupplemented human plasma samples. This procedure and a second procedure, method 2 (University of Arizona), were used to evaluate the stabilizing effects of DTT on patients' samples.

\section{Method 1}

AA, dehydroascorbic acid (DHAA), and DTT were purchased from Sigma Chemical Co., St. Louis, MO. The acetonitrile was "HPLC" grade. The AA, examined by proton magnetic resonance, contained $<0.1 \%$ impurities, including those related to $\mathrm{AA}$ or the degradation products of AA. Human plasma was prepared by Alpha Therapeutic Corporation and purchased from Western States Plasma Corp., Fallbrook, CA, in 650-g lots. This plasma was obtained by plasmapheresis from human blood to which $50 \mathrm{~mL}$ of a solution of sodium citrate per $500 \mathrm{~mL}$ of blood (final concentration, $5 \mathrm{~g} / \mathrm{L}$ ) was added. The processed plasmo was stored at $-70^{\circ} \mathrm{C}$ until used.

In the stability studies, supplemented human plasma extracts containing AA were analyzed with a Model 5560 chromatograph equipped with a Model 402 data station, a UV-200 variable-wavelength detector, a Model 8085 autosampler (all from Varian Associates, Sunnyvale, CA), and a Model LC-2 electrochemical detector (Bioanalytical Systems Inc., West Lafayette, IN).

The supplemented plasma (lots 58-61) was prepared as follows: after slowly thawing a 650-g package of plasma at room temperature overnight, we removed any insoluble material by filtration through cheesecloth. This yielded 595 $\mathrm{mL}$ plasma, to which we added $611 \mathrm{mg}$ DTT. The resulting solution was divided into four 148-mL lots. We supplemented three of the lots with AA by adding $\sim 0.3,0.75$, or $1.0 \mathrm{~mL}$, respectively, of a $1.01 \mathrm{~g} / \mathrm{L}$ AA stock solution. The fourth lot served as a blank for assessing the presence of possible substances interfering with the measurement methods. Each plasma lot was distributed in $1.3-\mathrm{mL}$ volumes into 5 . $\mathrm{mL}$ serum vials. The vials were then loosely capped with split serum stoppers, frozen, and freeze-dried. The stoppers, which were closed automatically after the samples were completely freeze-dried and the vacuum had been released, were secured with metal caps and stored at either -25 or $-80^{\circ} \mathrm{C}$. The process of filtering, supplementing, sampling, and freezing was completed within $3 \mathrm{~h}$. Each sample could

\footnotetext{
4 Identification of any commercial product does not imply recommendation or endorsement by the National Bureau of Standards, nor does it imply that the material or equipment identified is necessarily the best available for the purpose.
} 
be reconstituted to its original volume by adding $1.2 \mathrm{~mL}$ of distilled water.

To extract the AA from the freeze-dried plasma samples we added $1.5 \mathrm{~mL}$ of distilled water to each sample. After the material completely dissolved, we transferred the entire solution to a 4-mL screw-cap vial containing $160 \mu \mathrm{L}$ of 400 g/L aqueous MPA, capped the vial, and vortex-mixed for 15 s. We then added $400 \mu \mathrm{L}$ of HPLC-grade acetonitrile, capped the sample, and vortex-mixed for $15 \mathrm{~s}$. (This step improved protein aggregation and prevented precipitation of material on the top of the HPLC column.) After centrifuging the resulting suspension $\left(1000 \times \mathrm{g}, 30 \mathrm{~min}, 4^{\circ} \mathrm{C}\right)$, we transferred the supernatant fluid to $1.8-\mathrm{mL}$ vials, placed them in an autosampler, and flushed $300 \mu \mathrm{L}$ of sample through a 20 $\mu \mathrm{L}$ sampling loop (to eliminate carryover from the previous sample).

The samples were analyzed by a modification of the method of Grun and Loewus (6) with a $300 \times 7.8 \mathrm{~mm}$ Aminex HPX87H cation-exchange column (Bio-Rad Laboratories, Richmond, $\mathrm{CA}$ ). The column was equilibrated at $30{ }^{\circ} \mathrm{C}$ with $0.1 \mathrm{~mol} / \mathrm{L}$ formic acid containing $50 \mathrm{~mL}$ of acetonitrile per liter, at a flow rate of $0.5 \mathrm{~mL} / \mathrm{min}$. We added the acetonitrile to prevent bacterial growth and improve peak shape.

The chromatogram was developed isocratically and the eluted components were detected spectrophotometrically at $265 \mathrm{~nm}$ and electrochemically at $+0.7 \mathrm{~V}$, in the oxidation mode. The amount of AA was determined by peak-height measurement (electrochemical determinations) and by peak integration (ultraviolet absorbance and electrochemical determinations).

\section{Method 2}

Ascorbic acid ("Gold Label") was purchased from Aldrich Chemical Co., Milwaukee, WI. DTT, EDTA, chloroacetic acid, and MPA were purchased from Sigma Chemical Co. Octyl sodium sulfate was purchased from Eastman Kodak Co., Rochester, NY.

The HPLC instrumentation consisted of a Model 6000A pump (Millipore, Waters Chromatography Div., Milford, MA), a Model 7125 sampling valve with a $25-\mu \mathrm{L}$ sampling loop (Rheodyne Inc., Cotati, CA), a Model 3390A integrator (Hewlett Packard Co., Palo Alto, CA), a Model LC-4A electrochemical detector (Bioanalytical Systems Inc.), and a $250 \times 4.6 \mathrm{~mm}$ Ultrasphere column (Beckman Instruments Inc., Fullerton, CA) packed with 5- $\mu$ m octadecylsilane particles. All chromatographic solvents were filtered through a $0.2-\mu m$ (pore size) Nylon-66 filter (Rainin Instrument Co. Inc., Woburn, MA) before use.

All standard solutions of AA were either refrigerated or stored on ice until analysis. We prepared the standard solutions at concentrations that bracketed the expected range of concentrations in clinical samples by making appropriate immediate dilutions of a stock solution $(1 \mathrm{~g} / \mathrm{L}$ in an aqueous solution of MPA, $30 \mathrm{~g} / \mathrm{L}$ ). Blank plasma was supplemented with a similar stock solution. The AA standard curve was determined by transferring $100 \mu \mathrm{L}$ of supplemented or unsupplemented plasma to a $1.5-\mathrm{mL}$ "microfuge" tube containing $900 \mu \mathrm{L}$ of a cold, freshly prepared solution of MPA ( $30 \mathrm{~g} / \mathrm{L})$ and DTT ( $1 \mathrm{~g} / \mathrm{L})$. After standing 10 $\mathrm{min}$, the samples were centrifuged $(15 \mathrm{~min}, 11600 \times \mathrm{g}$ ) and a $25-\mu \mathrm{L}$ portion of the supernate was analyzed by HPLC.

Clinical samples of human plasma were prepared for storage at $-70^{\circ} \mathrm{C}$ by adding $100 \mu \mathrm{L}$ of plasma to $300 \mu \mathrm{L}$ of the MPADTT solution, mixing, and promptly freezing at
$-70{ }^{\circ} \mathrm{C}$. At the time of analysis we added $600 \mu \mathrm{L}$ of a freshly prepared $30 \mathrm{~g} / \mathrm{L}$ solution of MPA to the sample, thawed the sample, mixed well, centrifuged (11 $600 \times \mathrm{g}, 15 \mathrm{~min})$, and analyzed $25 \mu \mathrm{L}$ of the supernate by HPLC.

The analytical column and the electrochemical detector were conditioned (passivated) with an aqueous solution of $\mathrm{Na}_{4}$ EDTA $(2.55 \mathrm{~g} / \mathrm{L})$ at a flow rate of $1 \mathrm{~mL} / \mathrm{min}$ for $30 \mathrm{~min}$ before analyzing for AA. We used isocratic elution at a flow rate of $0.5 \mathrm{~mL} / \mathrm{min}$ at ambient temperature with a column that was equilibrated with a mobile phase consisting of 14.1 $\mathrm{g}$ of chloroacetic acid, $4.65 \mathrm{~g}$ of $\mathrm{NaOH}, 0.85 \mathrm{~g}$ of Na,EDTA, and $200 \mathrm{mg}$ of octyl sodium sulfate in $1 \mathrm{~L}$ of distilled water. The electrochemical detector was set at $+0.7 \mathrm{~V}$ and the amount of AA was quantified by peak-height measurement and electronic peak integration.

The plasma samples for use in the study comparing methods 1 and 2 were processed within $1 \mathrm{~h}$. We drew $15 \mathrm{~mL}$ of blood into heparinized, foil-wrapped tubes and centrifuged immediately. Seven milliliters of plasma was prompt. ly removed and $250 \mu \mathrm{L}$ of a solution containing $1.2 \mathrm{mg}$ DTT (final concentration, $0.17 \mathrm{~g} / \mathrm{L}$ ) and $125 \mathrm{mg}$ MPA (final concentration, $17.2 \mathrm{~g} / \mathrm{L}$ ) was added. The samples, divided into $1.3-\mathrm{mL}$ aliquots, were stored at $-80^{\circ} \mathrm{C}$ until analyzed.

For analysis by method 1 , we added $1.3 \mathrm{~mL}$ of sample to $160 \mu \mathrm{L}$ of a $400 \mathrm{~g} / \mathrm{L}$ solution of MPA and mixed for $15 \mathrm{~s}$, then added $400 \mu \mathrm{L}$ of acetonitrile, mixed for $15 \mathrm{~s}$, centrifuged, and analyzed the supernatant fluid for $\mathrm{AA}$ as described above. For analysis by method 2 the protocol previously described was followed. Each of the split samples from each patient was analyzed in duplicate.

\section{Results}

The utilization of DTT as an antioxidant for AA has been well documented $(3,7,8)$. Recent studies at NBS demonstrated that DTT is also an effective long-term preservative for AA (3). In some of the analyses for AA by $\operatorname{HPLC}(3,8)$ an aminosilane column was used, which resolved AA and DTT. We found it difficult to obtain reproducible chromatographic separations with different aminosilane columns. We also found it difficult to prevent degradation of the AA during the separation process on an aminosilane column without resorting to elaborate sparging and the use of antioxidants in the HPLC solvent (3). We circumvented these problems by using the HPX87H or an ODS 5- $\mu \mathrm{m}$ particle column. AA is not destroyed on these columns and AA is resolved from MPA, uric acid, and DTT, all of which produce an electrochemical response and could contribute to errors in measurement if they are not completely separated from AA.

Measurement of AA in plasma with an ultraviolet detector is difficult because there are several intense ultravioletabsorbing peaks that elute very close to $\mathrm{AA}$, and large amounts of sample must be used in order to obtain a sufficient signal for AA quantification. The use of an electrochemical detector results in increased sensitivity and selectivity (9) as compared with the ultraviolet technique. However, the DTT also has a strong electrochemical signal at the same voltage as AA (3). Because in our experience some ionpairing methods do not adequately resolve DTT from AA, it was necessary to select an HPLC method of analysis that would resolve these compounds. Methods 1 and 2 both give baseline resolution between uric acid, DTT, and AA (Figures 1 and 2), and method 1 also resolves two additional trace materials (Figure 1) that are present in AA-dêpleted plasma.

The range and sensitivity of the electrochemical method 
are particularly noteworthy: no more than $500 \mathrm{ng}$ of AA can be quantified under the conditions of these studies. It is also possible with a favorable signal/noise ratio to measure as little as $2 \mathrm{ng}$ of AA (Figure 2A). For method 1 the amount of AA routinely analyzed varied from 40 to $400 \mathrm{ng}$ in $20 \mu \mathrm{L}$ of sample and for method 2,5 to $30 \mathrm{ng}$ in $20 \mu \mathrm{L}$ of sample (Figure 2). Furthermore, analysis by method 2 is routinely completed in $<10 \mathrm{~min}$.

\section{Stability of the Ascorbic Acid during Automated Analysis}

All of the analyses done by method 1 were performed with an autosampler during $\sim 24 \mathrm{~h}$. Under these conditions, the stability of AA at room temperature becomes important (1, 2,10 ). We evaluated this variable by measuring the area of the chromatographic peaks absorbing in the ultraviolet seen on chromatographing standard solutions containing DTT. For periods up to $18 \mathrm{~h}$, little or no deterioration of the standard AA solutions at $21^{\circ} \mathrm{C}$ was detected, an observation supported by the fact that the relation between the concentration of AA and the area of the AA HPLC peak was linear from 2 to $20 \mathrm{mg} / \mathrm{L}$ and closely approached the origin (slope = 49124 arb. units, intercept $=817, r^{2}=0.9999, \mathrm{n}=6$ ). When stored at $4{ }^{\circ} \mathrm{C}$, these solutions remained stable for several weeks, after which new standards were prepared.

For standards stored at $4^{\circ} \mathrm{C}(\operatorname{method} 2)$ the AA measured remained constant for the duration of the experiment. The concentration curve for these standard solutions was linear from 5 to $20 \mathrm{mg} / \mathrm{L}$, intersecting the origin with a slope of 57.900 and a correlation coefficient of 0.9992 . The standards and samples analyzed by this method were never stored at room temperature.

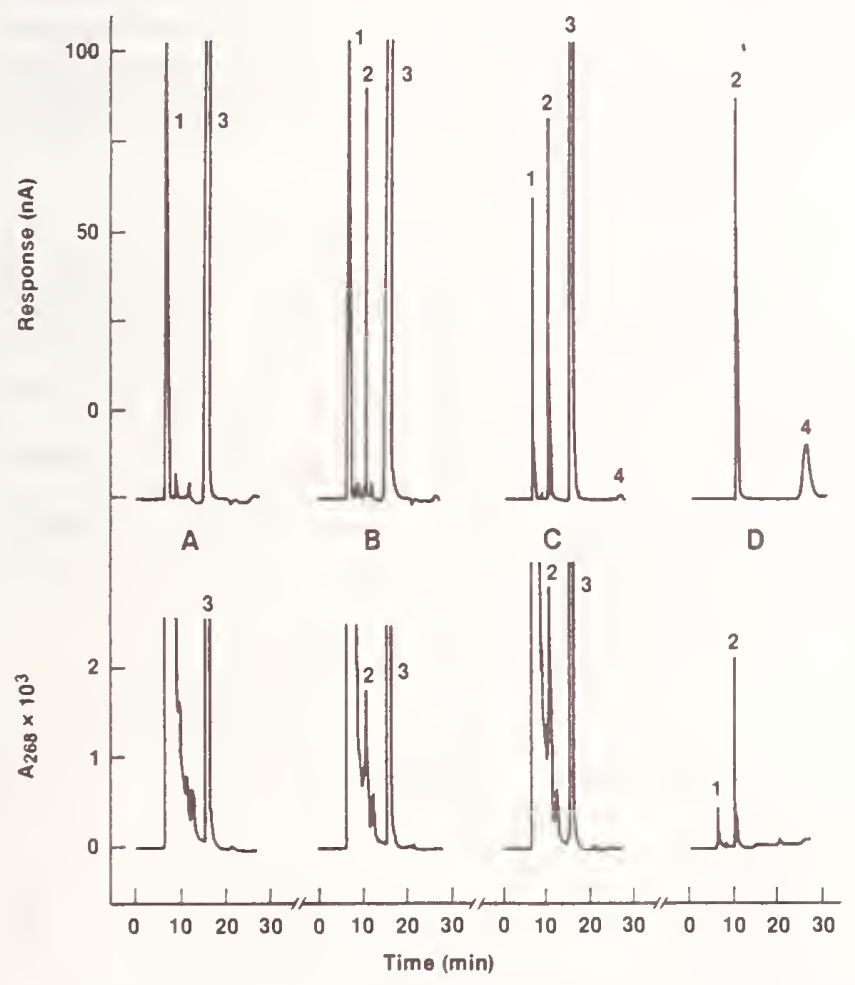

Fig. 1. Separation of ascorbic acid in human plasma on an HPX87H column (method 1)

$A$, unsupplemented plasma; $B$, plasma with added $A A, 4.80 \mathrm{mg} /$; $C$, plasma of subject 1 (Table 4) assayed on day 52; D. AA standard, $4.99 \mathrm{mg} / \mathrm{L}$, in acotonitrile/ water (3/1 by vol).

Upper traces are electrochemical chromatograms; lower traces are uttraviolet chromatograms at 268 nm. Peak identity: 1, MPA; 2, AA; 3, uric acid; 4, DTT
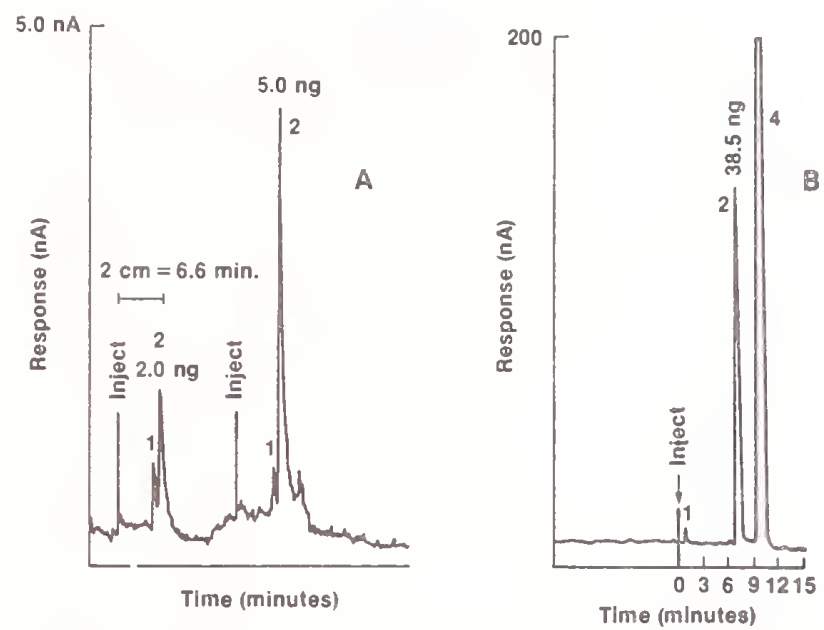

Fig. 2. Separation of ascorbic acid on an octadecylsilane column $(\operatorname{method} 2)$

A, standard solutions; $B$, human plasma. Peak identity as in Fig. 1

\section{Stability of the Electrochemical Detector}

Of primary consideration was the time-dependent variation in the sensitivity of the electrochemical detector. Method 1 was characterized by a time-dependent decrease in the detector sensitivity, which varied from one analysis to another. This was demonstrated by measuring, in duplicate, a series of standards (three different concentrations) at the beginning and at the end of an experiment. Each set of measurements was plotted separately. The ultraviolet $a b$ sorbance of the standards remained constant throughout the experiment (Figure $3 B$ ), whereas the electrochemical response decreased by a constant value over the entire concentration range, resulting in parallel curves displaced by a constant value (Figure $3 A$ ). Additionally, the standard of intermediate concentration $(4.2 \mathrm{mg} / \mathrm{L})$ was assayed after every two plasma samples (three additional times), and the results decreased steadily throughout the experiment, indicating a continuous change in the sensitivity of the electrochemical detector.

In contrast, method 2 was characterized by a timedependent increase in detector sensitivity; that is, the slope of the standard curve increased and the intercept remained constant over a 4-h period (Figure 3C). Thus, for both methods, frequent standards were run, to correct for variations in response of the electrochemical detector.

Stability of Ascorbic Acid in Human Plasma Supplemented with Dithiothreitol

Okamura (7) demonstrated that DTT reduces DHAA, so that total AA in plasma can be measured. To test the utility of using this reagent for reducing plasma DHAA and preserving plasma $\mathrm{AA}$ for extended periods of time, we modified pools of AA-depleted human plasma by adding DTT to give a concentration of $1 \mathrm{~g} / \mathrm{L}(5.3 \mathrm{mmol} / \mathrm{L})$ and subsequently supplemented with AA to give concentrations between 2 and $16 \mathrm{mg} / \mathrm{L}$ in plasma. Both freeze-dried and frozen supplemented 1.3-mL samples of plasma were prepared from these pools and stored at $-80^{\circ} \mathrm{C}$ for as long as 18.5 months. The AA content of these samples, measured at the indicated time intervals, is summarized in Table 1. Randomly selected samples from each of lots $37,38,45$, and 46 were analyzed in duplicate at the time intervals indicated in Table 1. These values were within one standard 

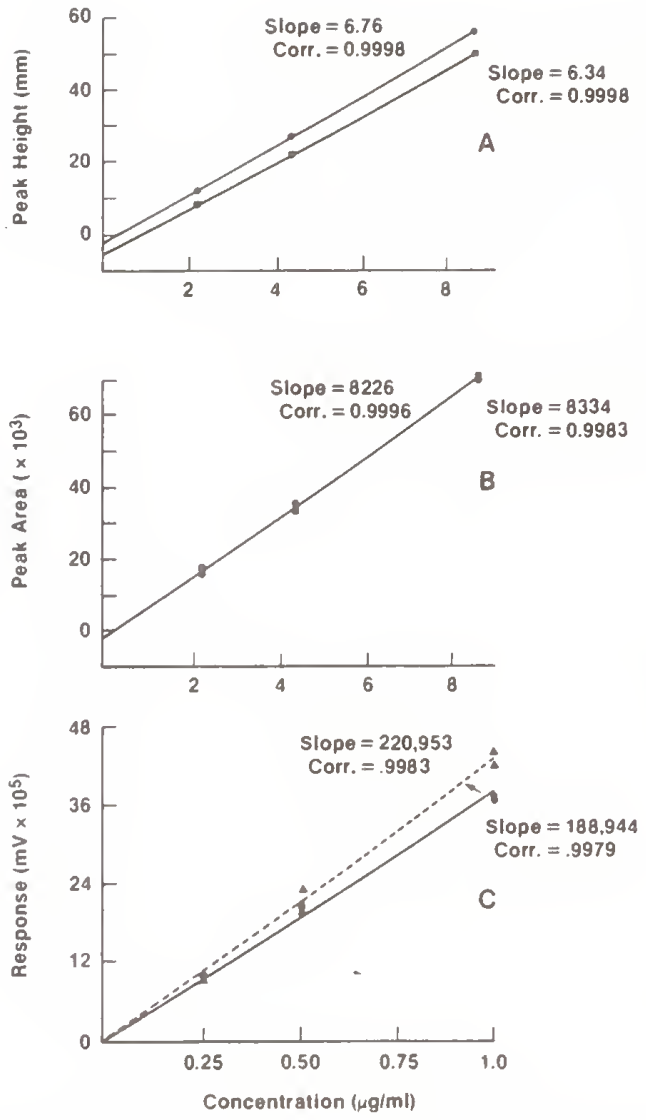

Fig. 3. Concentration-response curves for standard solutions of ascorbic acid as a function of storage time

A. ultraviofet detection at $268 \mathrm{~nm}$, method 1; B, electrochemical detection at 0.700 $\mathrm{V}$, method 1: $C$, electrochemical detection, at $0.700 \mathrm{~V}$, method 2 . The symbols represent the following time periods: $\longrightarrow, 0$ time; $\longrightarrow, 4 \mathrm{~h} ; x-. . \times, 18 \mathrm{~h}$

deviation of the amount gravimetrically added to the original plasma pool. This indicates that the AA is not oxidized in the presence of DTT under these storage conditions. Lots 58-61 constituted a second group of samples, which was prepared and freeze-dried. This group included an unsupplemented pool of plasma and three pools of plasma supplemented to contain $\sim 2,4.8$, and $6.7 \mathrm{mg}$ of added AA per liter of plasma. Four samples from each pool were analyzed in duplicate two weeks after the samples were prepared, to confirm the initial AA concentration. The plasma AA concentration of samples randomly selected from these pools was also measured at the time intervals indicated in Table 1. The results complement and support the conclusion that DTT indeed protects and preserves the integrity of AA in human plasma for at least 66 weeks when stored at $-80^{\circ} \mathrm{C}$. Finally, AA was never detected in any of the unsupplemented plasma samples (lot 59, Figure 1A).

The utility of this method for preparing reference materials for the measurement of AA in plasma is further supported by the results obtained in two unpublished collaborative studies (lots 37, 45, and 46; and lots 58,60, and 61) cosponsored by NBS and The National Cancer Institute (Table 2). The values reported in these collaborative studies, which were obtained with a variety of methods, show acceptable agreement with the measurements made at NBS.

\section{Mechanism of Action of DTT}

DTT completely reduces DHAA either in plasma or in a mixture of acetonitrile and water ( $75: 25$ by vol). The reac-
Table 1. Stablilty of Ascorbic Acid in Freeze-Drled and Frozen Plasma

Weeks stored

$\begin{array}{ccccccccc}\text { Plasma lot } & 0^{\circ} & 2^{\circ} & 6^{c} & 12^{c} & 27^{\circ} & 57^{c} & 66^{c} & 80^{c} \\ 37 & 6.50^{\circ} & & & & 6.51 & 6.27 & & \\ 38 & 15.60 & & & & 15.63 & 15.69 & & 15.21 \\ 45 & 8.80 & & & & 8.80 & 9.11 & & \\ 46^{\prime} & 8.80 & & & & 8.54 & 8.80 & & \\ 58 & 6.7 & 6.30 & 6.41 & 6.27 & 6.46 & 6.30 & 6.58 & \\ 60 & 2.0 & 1.82 & 1.85 & 1.81 & 1.74 & 1.83 & 1.83 & \\ 61 & 4.8 & 4.80 & 5.12 & 4.98 & 4.47 & 4.94 & 4.65 & \end{array}$

" Values for lots 37-46 represent gravimetric additions; values for lots 58 61 , at 0 time only, represent estimated concentrations.

'Means of eight measurements.

${ }^{c}$ Means of four measurements.

${ }^{\circ}$ Values for lots 37-46 are means of eight measurements and for lots 58-61 means of four measurements.

- AA concentration, $\mathrm{mg} / \mathrm{L}$. The $\mathrm{CV}$ for each value in this table ranges between $0.25 \%$ and $4.43 \%$.

'Lot 46 was frozen only: all other lots were freeze-dried.

Table 2. Comparison of Round-Robln AA Values and Values Obtained by NBS (mg/L)

\begin{tabular}{|c|c|c|c|c|c|c|}
\hline \multirow[b]{2}{*}{ Plasma lot } & \multicolumn{3}{|c|}{ Round-robln results ${ }^{*}$} & \multicolumn{3}{|c|}{ NBS reaults ${ }^{b}$} \\
\hline & Mean & $\mathbf{S D}^{c}$ & $\mathbf{n}$ & Mean & $\mathrm{SD}^{\circ}$ & $\mathbf{n}$ \\
\hline 37 & 6.34 & 1.09 & 14 & 6.33 & 0.21 & 13 \\
\hline 45 & 9.34 & 1.55 & 14 & 9.04 & 0.42 & 16 \\
\hline $46^{\alpha}$ & 9.25 & 1.07 & 14 & 8.73 & 0.26 & 16 \\
\hline 58 & 5.30 & 1.29 & 10 & 6.30 & 0.21 & 30 \\
\hline 60 & 1.72 & 0.46 & 10 & 1.81 & 0.08 & 30 \\
\hline 61 & 4.22 & 0.97 & 10 & 4.83 & 0.13 & 30 \\
\hline
\end{tabular}

Pound-robin measurements on lots 38,45 , and 46 were completed 13 weeks after the samples were prepared and the round-robin measurements on lots 58,60 , and 61 were completed four weeks after sample preparation. Each round robin represented an independent set of duplicate measurements on two difierent samples by each of seven and five laboratories, respectively.

${ }^{b}$ Data represent an aggregate mean of all data acquired in the stability studies summanized in Table 1.

${ }^{c}$ Standard deviation of a single measurement.

${ }^{\circ}$ Lot 46 was frozen only; all other lots were freeze-dried.

tion is complete within $60 \mathrm{~min}$ (compare the 1- and 4 -h values in Table 3 ). If MPA is added to the DTT before the plasma is added, the reduction still occurs (Table 2 and Figure 4). Thus by measuring the AA both before and after addition of the reducing agent, DTT, it is possible to measure the AA and DHAA differentially in biological samples by an electrochemical method. However, it is still impossible to determine by this method whether the DHAA was initially present in the plasma or was formed during removal of the blood cells.

\section{Table 3. Conversion of DHAA by DTT}

\begin{tabular}{|c|c|c|}
\hline \multirow[b]{2}{*}{ Time of analysis } & \multicolumn{2}{|c|}{ Ascorblc acld, mg/L" } \\
\hline & $1 \mathrm{~h}$ & $4 \mathrm{~h}$ \\
\hline \multicolumn{3}{|l|}{ Experiment 1} \\
\hline Solvent ${ }^{b}$ & 0 & 0 \\
\hline Solvent $+\mathrm{DT}$ & 8.7 & 8.8 \\
\hline Plasma & 0 & 0 \\
\hline Plasma + DTT & 9.1 & 8.8 \\
\hline \multicolumn{3}{|l|}{ Experiment 2} \\
\hline Plasma & 0 & 0 \\
\hline Plasma + DT & 6.3 & 6.8 \\
\hline MPA + DTT + plasma & 6.1 & 6.7 \\
\hline
\end{tabular}


We evaluated the effect of DTT on AA in blood by collecting into heparinized tubes two blood samples from a patient with very low concentrations of AA in his plasma. One of the blood samples $(9.5 \mathrm{~mL})$ was collected into $0.5 \mathrm{~mL}$ of a solution containing $15 \mathrm{mg}$ DTT; the other blood sample served as a control. AA was added to each blood sample to give a concentration of $7 \mathrm{mg} / \mathrm{L}$, the plasma was prepared, and the AA was measured by method 2. Five replicates of each measurement were made. The results indicate that there was no destruction of AA in either sample [plasma 7.2 (SD 0.6) $\mathrm{mg} / \mathrm{L}$, and blood 7.5 (SD 0.5) $\mathrm{mg} / \mathrm{L}$. Thus one can collect blood samples directly into DTT solutions without affecting the ability to remove the cells.

\section{Assessment of the Stability of Patients' Plasma Ascorbic Acid Presenved in DTT and MPA}

The utility of a method for stabilizing AA in human plasma depends on the demonstration that it is efficient and that the same results can be obtained by two or more independent methods. A comparison of the results obtained by each method ( 1 vs 2 ), during the same time period, for the indicated lots of reference plasma samples showed, for lot 37 ( 6.00 vs $6.15 \mathrm{mg} / \mathrm{L}$ ), 45 ( 8.80 vs $8.95 \mathrm{mg} / \mathrm{L}$ ), 46 ( 8.80 vs 9.15 $\mathrm{mg} / \mathrm{L}), 58$ (6.27 vs $6.00 \mathrm{mg} / \mathrm{L}), 60$ (1.81 vs $2.05 \mathrm{mg} / \mathrm{L}$ ), and 61 (4.98 vs $4.90 \mathrm{mg} / \mathrm{L}$ ). Evidently, the two methods yield equivalent results. Furthermore, comparison of the experimental values for plasma lots-37, 45, and 46 with the gravimetric values in Table 1 also indicates that the methods are accurate. On this basis, a series of 11 blood samples from normal subjects was collected, centrifuged, and the plasma preserved within $1 \mathrm{~h}$ as described in the Methods section. This group consisted of five men and six women,

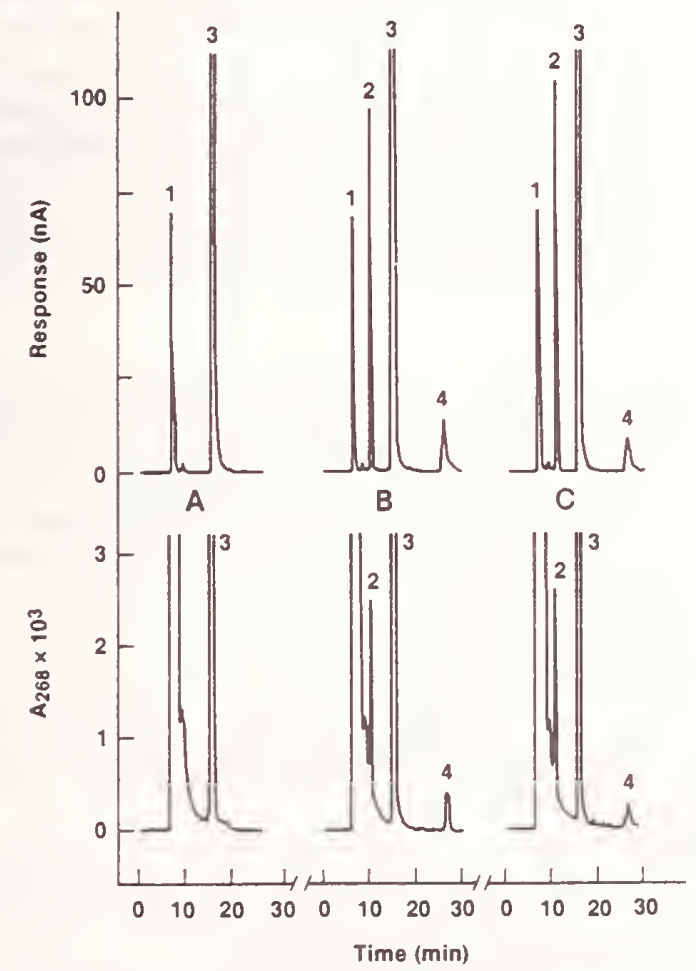

Fig. 4. Effect of DTT and MPA on the conversion of DHAA to ascorbic acid in human plasma

A. DHAA (7 mg/L) in plasma; $B$, DHAA (7 mgL) in plasma after exposure to DTT (1 $\mathrm{g} /$ ) for $1 \mathrm{~h} ; C$, DHAA $(7 \mathrm{mg} / \mathrm{L})$ in plasma atter exposure to DTT $(1 \mathrm{~g} / \mathrm{L})$ and MPA (20 gh) for $1 \mathrm{~h}$. Upper traces are electrochemical chromatograms; tower traces are ultraviolet chromatograms at $268 \mathrm{~nm}$. Peak identity as in Fig. 1
Table 4. Ascorbic Acid Concentrations (mg/L) in Plasma of 11 Normal Subjects ${ }^{a}$

AA concentration, $\mathrm{mg} / \mathrm{L}$

\begin{tabular}{|c|c|c|c|c|c|c|}
\hline \multirow{2}{*}{$\begin{array}{l}\text { Sex of } \\
\text { subject }\end{array}$} & \multicolumn{4}{|c|}{ Days stored ${ }^{b}$} & \multirow[b]{2}{*}{ Mean } & \multirow[b]{2}{*}{ SD $^{c}$} \\
\hline & 23 & 32 & 51 & 52 & & \\
\hline$F$ & 7.8 & 7.3 & 7.8 & 6.9 & 7.5 & 0.4 \\
\hline $\mathbf{F}$ & 19.9 & 19.2 & 18.7 & 18.9 & 19.2 & 0.5 \\
\hline $\mathbf{F}$ & 8.7 & 8.4 & 8.6 & 8.5 & 8.6 & 0.1 \\
\hline $\mathbf{M}$ & 10.8 & 10.8 & 10.9 & 10.8 & 10.8 & 0.0 \\
\hline$F$ & 12.1 & 11.8 & 12.3 & 12.0 & 12.1 & 0.2 \\
\hline$F$ & 7.6 & 8.1 & 7.7 & 8.2 & 7.9 & 0.3 \\
\hline$M$ & 8.7 & 8.8 & 8.5 & 8.6 & 8.7 & 0.1 \\
\hline $\mathrm{M}$ & 9.8 & 10.0 & 9.5 & 10.4 & 9.9 & 0.3 \\
\hline$M$ & 15.1 & 15.9 & 14.9 & 15.4 & 15.3 & 0.4 \\
\hline$M$ & 7.9 & 7.9 & 7.6 & 8.0 & 7.9 & 0.1 \\
\hline $\mathbf{F}$ & 16.3 & 16.5 & 16.3 & 16.0 & 16.3 & 0.2 \\
\hline
\end{tabular}

age 18 to $40 \mathrm{y}$.

${ }^{b}$ The samples on days 23 and 51 were analyzed by method 1 and the samples on days 32 and 52 were analyzed by method 2. Each value represents the mean of duplicate analyses.

' Standard deviation of a single measurement.

ages 18-40. The plasma samples were prepared at the University of Arizona, frozen at $-80^{\circ} \mathrm{C}$, shipped in solid $\mathrm{CO}_{2}$, and stored at $-80^{\circ} \mathrm{C}$ until the $A_{\mathrm{L}} \mathrm{A}$ was measured. The results are summarized in Table 4. The CV for these determinations was between $0.1 \%$ (subject 1 ) and $5.3 \%$ (subject 10). The size of the stanciard deviation does not appear to be concentration dependent (compare subject 1 with 9) or method dependent (same subjects). Furthermore, the samples appear to be stable for no less than 52 days, thus establishing the utility of this method for collecting and storing patients' plasma for AA measurement.

\section{Discussion}

Measuring AA in plasma is useful in the diagnosis of deficiency syndromes, the assessment of human nutritional status, and in confirming subject adherence (compliance) to experimental protocols such as chemoprevention and chemotherapy studies (13). For large, multilaboratory studies, it is essential to have suitable stable reference materials to assess the quality control, precision, and accuracy of participating laboratories. The human plasma reference material that we describe here can be prepared by a central laboratory for the assessment of interlaboratory and intralaboratory performance or can be prepared in the individual laboratory and used for an extended period of time to assess intralaboratory quality control.

The use of reducing agents to convert DHAA to $\mathrm{AA}$ is well documented, particularly with respect to methods in which the AA was derivatized before spectrophotometric measurement $(12)$ as well as techniques involving $\operatorname{HPLC}(3,4,8,11)$. The utility of DTT as a reducing agent for DHAA in plasma was demonstrated by Okamura (7) and used for the measurement of AA in fruit juices $(4,8)$ and urine $(8)$. Okamura (7) noted that among the reducing agents tested, DTT exhibited reducing activity over a broader $\mathrm{pH}$ range than the other compounds examined, and in aqueous solution it was more stable than other reducing agents. It was superior to glutathione, mercaptoethanol, cysteine, homocysteine, and 2,3-dimercapto-1-propanol. By using DTT to convert DHAA to AA, Okamura was able to measure differentially DHAA and total plasma AA by assaying $\mathrm{AA}$ in the presence and absence of DTT. Since then, DTT was shown to be an effective antioxidant for the long-term preservation of AA in 
aqueous organic solution (3). These observations were extended to include plasma, which can be stored in the presence of DTT for more than one year at $-80^{\circ} \mathrm{C}$ without the AA undergoing any oxidation (Table 1). Blood can also be collected into a solution of DTT without causing coagulation. Therefore, DTT can be introduced immediately at the time of blood sampling, thus preventing any oxidation of $\mathrm{AA}$, either during the process of removal of blood cells or during subsequent storage. However, this procedure precludes the measurement of any native DHAA.

The differential content of DHAA and AA can be determined by first obtaining the plasma, treating it with MPA to stabilize it, and then measuring the plasma AA both in the absence and presence of DTT. The difference between these measurements represents the DHAA content. This follows from the observation that in the presence of MPA (20 $\mathrm{g} / \mathrm{L})$ and DTT ( $1 \mathrm{~g} / \mathrm{L}), \mathrm{DHAA}$ in plasma is converted to AA (Table 3 and Figure 4). This type of experiment was done with fruit juice $(4,8,11)$ and urine $(8)$.

The measurement of total AA in plasma preserved at $-80^{\circ} \mathrm{C}$ in DTT $(170 \mathrm{mg} / \mathrm{L})$ plus MPA $(20 \mathrm{~g} / \mathrm{L})$ and the stability of clinical samples preserved in this manner was evaluated on split samples from 11 subjects. The samples were stable for 52 days (Table 4) and detectable amounts of DTT were still present in the sample (Figure $1 \mathrm{C}$ ). We did not observe any significant consistent variation in the AA content of these samples, and these observations are consistent with the results reported by other investigators $(1,4)$. Thus DTT prevents oxidation of AA in blood samples without causing coagulation (at a concentration of $1 \mathrm{~g} / \mathrm{L}$ ), and in plasma for extended periods of time in the presence or absence of MPA and in either the frozen or freeze-dried state.

All of the colorimetric methods are based on either the direct oxidation of $\mathrm{AA}$ by a colorimetric reagent or the oxidation of AA to DHAA and the subsequent derivatization of DHAA to a material that can be measured colorimetrically (12). The lack of total specificity of these reactions can lead to significant errors in measurement. Because all of these methods involve the measurement of a reduced form of $\mathrm{AA}$, the presence of relatively large amounts of a reducing agent (DTT) can introduce a significant error. Okamura (7) solved this problem by reducing his AA samples with sulfhydryl reagents and then removing the sulfhydryl reagents by treating his samples with $N$-ethylmaleimide. Thus, reference AA samples treated with DTT can be used as standards for some colorimetric AA assays if the samples are first treated with a sulfhydryl reagent such as $N$ ethylmaleimide.

Using HPLC methods in conjunction with electrochemical detectors improves selectivity of AA determinations (8). The increase in selectivity as well as sensitivity over the ultraviolet detectors can be seen in the dual recording in Figures 1 and 4. It is apparent that the AA signal is not well resolved from other ultraviolet peaks, whereas baseline resolution was observed with electrochemical detection. Furthermore, the electrochemical method permits increases in sensitivity of at least two orders of magnitude as is demonstrated by method 2 (Figure 2).

The three HPLC methods that were used to analyze AA in the presence of DTT were chosen because they resolve DTT from AA. These include analysis on an aminosilane column in a previous study (3), an HPX87H column (method 1), and a reversed-phase column (method 2). Each of these systems resolves DTT, uric acid, and metaphosphoric acid from AA.
We were unable to obtain consistent results with different aminosilane columns and therefore, have adopted the two methods described herein.

Both HPLC methods are capable of measuring accurately the AA concentration in gravimetrically supplemented samples (37, 38, 45, and 46). The accuracy of these two methods was compared by analyzing the reference samples described in Tables 1 and 2 . The results are in good agreement, and because the unsupplemented plasma contained no electrochemically detectable material with the retention time of $\mathrm{AA}$, these results indicate that both methods accurately measure the AA in plasma. Furthermore, the use of two independent methods that are based on different mechanisms of separation (ion exchange vs paired ion chromatography) lends credence to the accuracy of the measurement and decreases the probability that interfering substances are present in the analyte peak.

With the knowledge that these methods were accurate within the limits of the two methods and that DTT reduced DHAA and preserved AA in supplemented plasma samples, we evaluated the plasma AA content of a group of normal subjects. The results of this study indicate that samples that are preserved with MPA (17 g/L) and DTT (170 $\mathrm{mg} / \mathrm{L})$ at $-80^{\circ} \mathrm{C}$ are stable for no less than 52 days and can be shipped transcontinentally in solid $\mathrm{CO}_{2}$. The values thus obtained indicate that in normal subjects the AA concentration in plasma can range from $\sim 7$ to $19 \mathrm{mg}$ of plasma per liter. Thus, a suitable assay procedure should include standards from subnormal concentrations of AA to concentrations comparable to $20 \mathrm{mg}$ of AA per liter of plasma. Using either of these separation techniques coupled with electrochemical detection, a laboratory can easily automate the analysis of six to eight clinical samples per hour.

We thank Ms. Margaret Kline and Dr. Robert Schaffer for their assistance in the preparation of the ascorbic acid-supplemented human plasma samples; Alison Culling-Berglund and Wendi Morfitt for their technical assistance; Dr. Robert Paule for his advice on the statistical analysis; and Dr. Willie May for coordination of the NBS/NCI Quality Assurance Program. This study was supported in part by the National Cancer Institute contract nos. Y01-CN-70645 (NBS) and NCI-CA 27502 (University of Arizona).

\section{References}

1. Bradley DW, Emery G, Maynard JD. Vitamin C in plasma: a comparative study of the vitamin stabilized with trichloracetic acid or metaphosphoric acid and the effects of storage at $-70^{\circ},-20^{\circ}, 4^{\circ}$, and $25^{\circ}$ on the stabilized vitamin. Clin Chim Acta 1973;44:47-52. 2. Baker JK, Kapeghian J, Verlangieri A. Determination of ascorbic acid and dehydroascorbic acid in blood plasma samples. J Liq Chromatogr 1983;6:1333-44.

3. Margolis SA, Black I. Stabilization of ascorbic acid and its measurement by liquid chromatography in nonfat dry milk. J Assoc Off Anal Chem 1987;70:806-9.

4. Lloyd LL, McConville JA, Warner FP, Kennedy JF, White CA. HPLC analysis of L-ascorbic acid (vitamin C) using a polymeric reversed-phase adsorbent. Liq Chromatogr Gas Chromatogr 1987;5:338-41.

5. Sauberlich HE, Green MD, Omaye ST. Determination of ascorbic acid and dehydroascorbic acid. In: Seib PA, Tolbert BM, eds. Ascorbic acid: chemistry, metabolism and uses [Review]. Washington DC: Am Chem Society, 1982:199-221.

6. Grun M, Loewus F. Determination of ascorbic acid in algae by high-performance liquid chromatography on strong cation-exchange resin with electrochemical detection. Anal Biochem 1983;130:191-8.

7. Okamura M. An improved method for determination of $L$ ascorbic acid and $\mathrm{L}$-dehydroascorbic in blood plasma. Clin Chim Acta 1980;103:259-68. 
8. Doner LW, Hicks KB. High performance liquid chromatographic separation of ascorbic acid, erythrobic acid, dehydroascorbic acid and diketogluconic acid. Anal Biochem 1981;115:225-30.

9. Pachla LA, Kissinger PT. Analysis of ascorbic acid by liquid chromatography with amperometric detection. Methods Enzymo 1979:62:15-24.

10. Rose RC, Nahrwold DL. Ascorbic acid analysis in biological fluid. Anal Biochem 1982;123:389-93.

11. Dennison DB, Brawley TG, Hunter GLK. Rapid high-perform- ance liquid chromatographic determination of ascorbic acid and combined ascorbic acid-dehydroascorbic acid in beverages. J Agric Food Chem 1981;29:927-9.

12. Bui-Nguyen MH. Ascorbic acid and related compounds. In: De Leenheer AP, Lambert WE, De Ruyter MGM, eds. Modern chromatographic analysis of the vitamins (Chromatographic Sci Ser, Vol 30). New York: Marcel Dekker Inc., 1985:267-301.

13. Jaffe GM. Vitamin C. In: Machlin LJ, ed. Handbook of vitamins: nutritional, biochemical and clinical aspects. New York: Marcel Dekker Inc., 1984: 199-244. 



\section{DETERMINATION OF VITAMIN $\mathrm{K}_{1}$ IN SERUM}

In this section a new approach to the detection of serum vitamin $K_{1}$ is described. This vitamin may have some involvement in the respiration of tumor cells. Details of the procedure and critical factors are derived from a manuscript accepted for publication in the Journal of Chromatography, Biomedical Applications. Listed below are some of the factors important to this analysis.

\section{Important Factors}

\section{Calibration and Sample Preparation}

- For the liquid-liquid extraction we recommend serum proteins be precipitated with two volumes of either ethanol or 2-propanol, and that three sequential extractions with four volumes of fresh hexane be used.

- Care should be taken to minimize losses through the extraction, solvent evaporation, solid-phase fractionation elution, and final evaporative concentration by careful washing of container walls and transfer pipets.

- Concentrations of calibration solutions for vitamin $\mathrm{K}_{1}$ should be assigned by spectrophotometry; we use a molar absorptivity of 41,000 at $242 \mathrm{~nm}$ for this determination.

- Stock solutions were stored refrigerated at a concentration of approximately $50 \mu \mathrm{g} / \mathrm{mL}$ and diluted just prior to use. The stock solutions appeared to be stable for at least 2 months.

- Use an internal standard; we used vitamin $\mathrm{K}_{2}$ (which is rare in most sera and is added to the protein precipitant solution).

- Care should be taken to prevent photodecomposition throughout the extraction and analysis.

\section{Chromatography}

- We recommend the use of a polymerically bonded $\mathrm{C}_{18}$ column $(25 \mathrm{x} 0.46 \mathrm{~cm})$ for the analytical separation. 
- An "on-line" reducer column (5 x $0.2 \mathrm{~cm}$ I.D.) and catalytic oxygen scrubber $(10 \times 0.46$ $\mathrm{cm}$ ) should be dry-packed using vibration from a column vibrator or an electric engraving tool.

- Great care should be taken in handling the catalyst during column preparation and in disposal since contact of organic solvents with even small amounts of the solid causes spontaneous combustion of the liquid.

- Best sensitivity is achieved with a xenon light-source fluorimeter.

For further information, please contact William MacCrehan at (301)975-3122. 


\title{
Determination of Vitamin $\mathbf{K}_{1}$ in Serum
}

\author{
William MacCrehan and Emil Schönberger
}

Vitamin $\mathrm{K}_{1}$ (phylloquinone) is a substituted naphthoquinone, required for proper blood clotting. Unfortunately, there is very poor agreement in the published literature on the measured "normal" serum concentration of $\mathrm{K}_{1}$, with reported values as low as $0.2 \mathrm{ng} / \mathrm{mL}$ and as high as $2 \mathrm{ng} / \mathrm{mL}$. Most of this disagreement is likely to be a result of poor measurement technology and lack of serum-based $K_{1}$ standards for the evaluation of method accuracy. The higher levels that are found in foods $(\mu \mathrm{g} / \mathrm{g})$ may be measured with LC using UV detection of the strong naphthoquinone absorption in the $240-260 \mathrm{~nm}$ range. Since serum concentrations of $\mathrm{K}_{1}$ are three orders of magnitude lower, more selective and sensitive detection is required for the clinical determination.

NIST has developed a new LC method for the determination of Vitamin $\mathrm{K}_{1}$ based on the "online" chemical reduction of the non-fluorescent quinone to the highly fluorescent hydroquinone. The reduction is done in a post-column catalytic reducer column packed with a platinum-on-alumina catalyst (10\% Pt) that uses an alcohol added to the mobile phase as the reducing agent. In the reduction reaction, the quinone is reduced by the solvent in the presence of the catalyst:

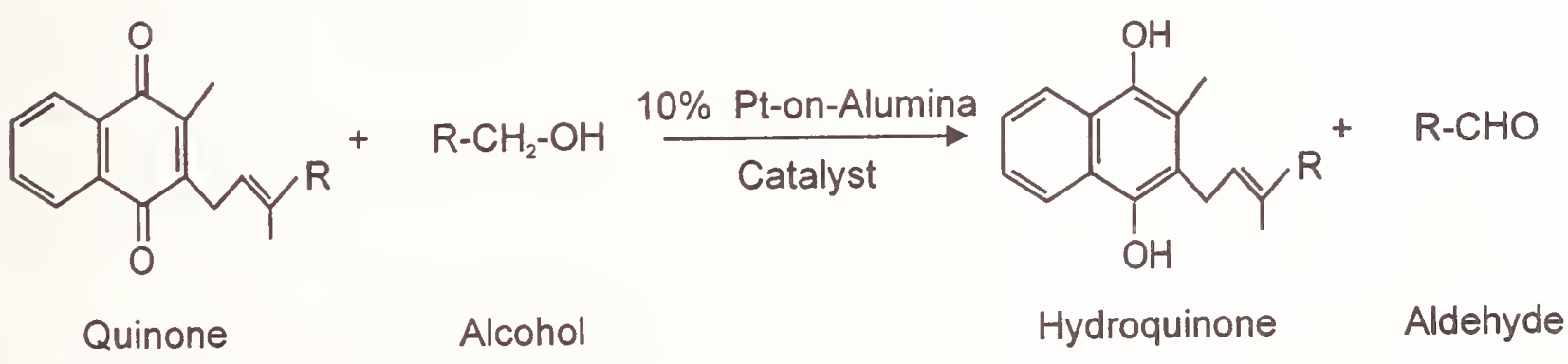

Figure 1: The Catalytic Reduction of the K Vitamins.

This is the same catalytic reduction approach that we previously developed for the removal of mobile phase oxygen prior to reductive electrochemical detection of nitropolyaromatic hydrocarbons \{Anal. Chem. 6, 284-286 (1988)\}. To apply this concept to the reduction of Vitamin $K_{1}$, we first evaluated the conditions required for quantitative reduction of the quinone functionality to the hydroquinone. The reductor was inserted ahead of the analytical column and the disappearance of the parent and product compounds were monitored by UV absorbance and fluorescence detection. 
The apparatus for the reductive/fluorescence LC detection of Vitamin $K_{1}$ in serum is shown in Figure 2. The chemical conversion to a single fluorescent product is nearly quantitative when either methanol or ethanol are used at a flow rate of $1 \mathrm{~mL} / \mathrm{min}$ for a $50 \times 2 \mathrm{~mm}$ catalyst column. We found that the catalyst column retained full activity for the 10 months of the study.

A second issue addressed in the method was the isolation of the Vitamin $\mathrm{K}_{1}$ from the other fluorescent matrix components that are co-extracted from sera. A liquid-liquid extraction and a column fractionation on a solid-phase extraction (SPE) cartridge were used to selectively isolate the Vitamin $\mathrm{K}_{1}$ before the final LC determination.

Serum Extraction and Fractionation Procedure for Vitamin $K_{1}$ Determination

Five hundred microliters of serum (warmed to room temperature and mixed, if necessary) is measured into a conical centrifuge tube. One milliliter of an approximately $1 \mathrm{ng} / \mathrm{mL}$ solution of $\mathrm{K}_{2}$ in 2-propanol is added by vortex mixing for 5 seconds. Three milliliters of hexane are added and the mixture is vortex mixed for 30 seconds. The layers are separated using centrifugation. The organic layer is drawn off and placed in an amber vial having a twoholed cap. The sample is dried under a current of dry $\mathrm{N}_{2}$ at $30-40{ }^{\circ} \mathrm{C}$. The residue is redissolved by careful washing with rotation in $0.5 \mathrm{~mL}$ of hexane for sorbing to the SPE cartridge.

Silica SPE cartridges (Baxter Enviroprep Inert SPE, $500 \mathrm{mg}$ packing) are used for the isolation of the Vitamins $\mathrm{K}_{1}$ and $\mathrm{K}_{2}$ from matrix fluorescent interferences. New cartridges are washed with $5 \mathrm{~mL}$ of hexane prior to sampling. The extract is then sorbed to the column, and fats and retinol are eluted using $10 \mathrm{~mL}$ of hexane, which is discarded. Elution of the $\mathrm{K}_{1} / \mathrm{K}_{2}$ fraction is done with $5 \mathrm{~mL}$ of $3 \%$ ether in hexane. The first $1 \mathrm{~mL}$ that elutes is discarded and then the subsequent $4 \mathrm{~mL}$ are collected for further processing. These volumes are critical to eliminate the fats and retinol eluted by the hexane (without losing the $\mathrm{K}$ vitamins) and to prevent collection of the interfering tocopherols, which elute following the collected $4 \mathrm{~mL}$ fraction. The eluant is dried under nitrogen, and the residue is redissolved in a volume of ethanol that is determined by the sensitivity of the fluorimetric detector. Volumes between $100 \mu \mathrm{L}$ and $500 \mu \mathrm{L}$ can be used. Fifty microliters are injected into the chromatograph for analysis. SPE cartridges may be reused by washing with $10 \mathrm{~mL}$ of the $3 \%$ ether followed by $10 \mathrm{~mL}$ of hexane.

\section{Rationale for the Procedure}

Like other lipid-soluble vitamins, the Vitamins $\mathrm{K}_{1}$ and $\mathrm{K}_{2}$ can be extracted from serum with hexane following denaturation of the proteins. We found that 2-propanol as protein precipitant provided better recovery and lower amounts of coextracted interferences than did ethanol. After a standard liquid-liquid vortex extraction, the hexane/2-propanol layer is drawn off the top and evaporated under inert gas to dryness. Care must be taken to remove all residual 2-propanol since it causes band broadening and loss of selectivity in the silica fractionation. The small volume of hexane used to redissolve the extract must be swirled around the container walls to maximize redissolution. 
An internal standard, added to the serum prior to extraction, is crucial in order to correct for volume changes and other losses in the procedure. For this serum work, we chose menaquinone - 4, Vitamin $\mathrm{K}_{2}$, which is identical to $\mathrm{K}_{1}$ except for saturation of two of the side chain double bonds. This $\mathrm{K}$ vitamin does not generally occur in human serum. The chromatographic behavior of $\mathrm{K}_{2}$ is nearly identical to $\mathrm{K}_{1}$ for both the fractionation and reverse-phase separation steps and thus serves as an excellent internal standard.

The crude liquid-liquid extract matrix is too complex for direct $\mathrm{K}_{1}$ determination since it contains excess coextracted fat and compounds that interfere with the LC-fluorescence determination, such as retinol and the tocopherols. Using the normal-phase SPE fractionation scheme outlined, retinol and most fats elute before the $\mathrm{K}$ vitamins using the hexane solvent. Tocopherols were found to elute in the fractions following the $\mathrm{K}$ vitamins. We found that an all-glass/fluorocarbon SPE cartridge did not present any interference from background fluorescence of the dialkyl phthlates found in plastic SPE cartridges. The syringe tube cartridge type conveniently holds the eluting solvents and provides a gravity flow rate of about $1 \mathrm{~mL} / \mathrm{min}$ with hexane. The concentration and volume of the ether in hexane mixture was found to be crucial for a good separation of the quinones from interfering matrix compounds.

\section{Chromatographic Determination}

For the analytical separation two columns were tested, a Zorbax ODS and a Vydac 201TP $C_{18}$ column $(25 \times 0.46 \mathrm{~cm})$. The Zorbax showed the higher retention and the Vydac showed much lower $\mathrm{k}^{\prime}$ values. We investigated the retention for both columns using methanol:ethanol solvent mixtures. The retention time is reduced as the ethanol concentration is increased; however, some asymmetry at high ethanol concentration is observed, possibly due to increased solvent viscosity. The best separation of the Vitamin $\mathrm{K}$ compounds was achieved with the Vydac column using $20 \%$ ethanol in methanol, with a flow rate of 1 $\mathrm{mL} /$ minute. With this separation, the two $\mathrm{K}$ vitamins are baseline resolved and well separated from early eluting interferences. In serum extracts a chromatographic peak with a k' that is twice the value of the $\mathrm{K}$ vitamins was observed and was judged to probably be a higher $n$ value menaquinone. Under these conditions, the chromatographic separation time is about 20 to 25 minutes in length, as shown in Figure 3. The fluorescence detection wavelengths were $242 \mathrm{~nm}$ for excitation and $430 \mathrm{~nm}$ for emission.

Calibration solutions were prepared by dissolving $\mathrm{K}_{1}$ and $\mathrm{K}_{2}$ in ethanol and value-assigned by spectrophotometry. The molar absorptivity of Vitamin $K_{1}$ that was used was 41,000 at 242 $\mathrm{nm}$. Stock solutions of about $40 \mu \mathrm{g} / \mathrm{mL}$ were stored in the dark in the refrigerator. The stock solutions appeared to be stable for at least 2 months. Calibration curves were evaluated in the range of 50 to $1000 \mathrm{ng} / \mathrm{mL}$ and found to be quite linear at the higher concentrations. There was some non-linearity at the lower concentrations that is similar to what we have found for $\beta$-carotene. 
Performance of the Method

We have carefully evaluated the recovery of both spiked analyte $\left(\mathrm{K}_{1}\right)$ and internal standard $\left(\mathrm{K}_{2}\right)$ in each of the procedural steps in the analysis. The overall recovery of extraction, fractionation, evaporation and reconstitution steps was found to be $70 \pm 34 \%$. Losses may occur in several ways. Perhaps most important are losses in the transfer of the small volumes of extract. Redissolution of the extract following the evaporation of the crude hexane extract must be done with several rinses and vortex mixing to assure complete dissolution of the fatty residue. The transfer of the evaporated crude extract to the SPE cartridge must be done with care to avoid liquid losses. Redissolution of the fractionated extract also requires washing and mixing since some fatty residue remains which is not rapidly soluble in the ethanol solvent. Losses by photodegradation are also possible, so all work should be done in amber or aluminum foil-covered vials. The absolute accuracy of the Vitamin $\mathrm{K}_{1}$ quantitation assumes similarity in the extraction and fractionation behavior (and losses) of the $\mathrm{K}_{1}$ and the $\mathrm{K}_{2}$ throughout the procedure. We have tested the method on two serum pools from the NIST/NCI round robin set. A value of $200 \mathrm{pg} / \mathrm{mL}$ was measured for Serum \#28 and 420 $\mathrm{pg} / \mathrm{mL}$ for Serum \#70. 

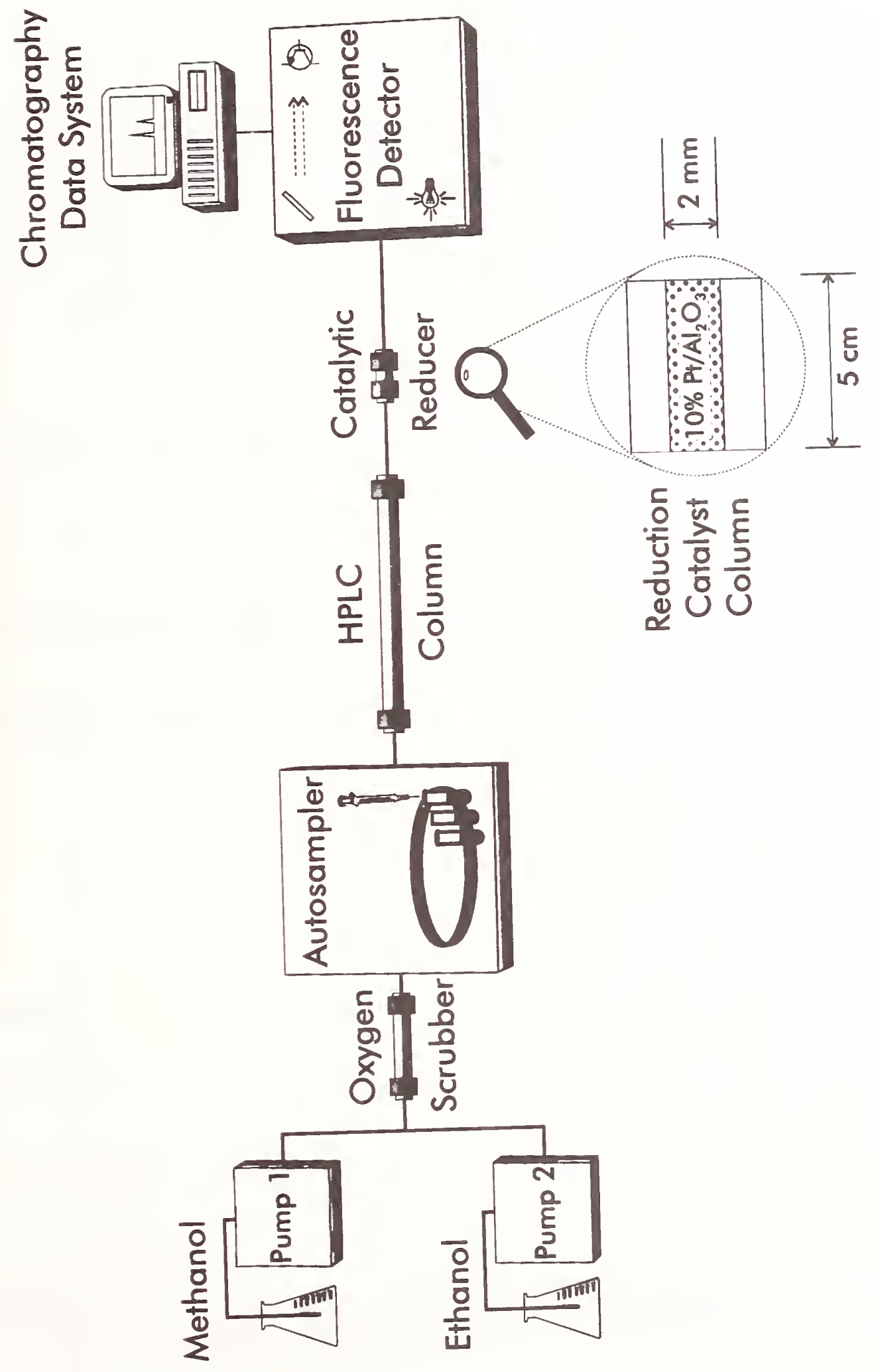


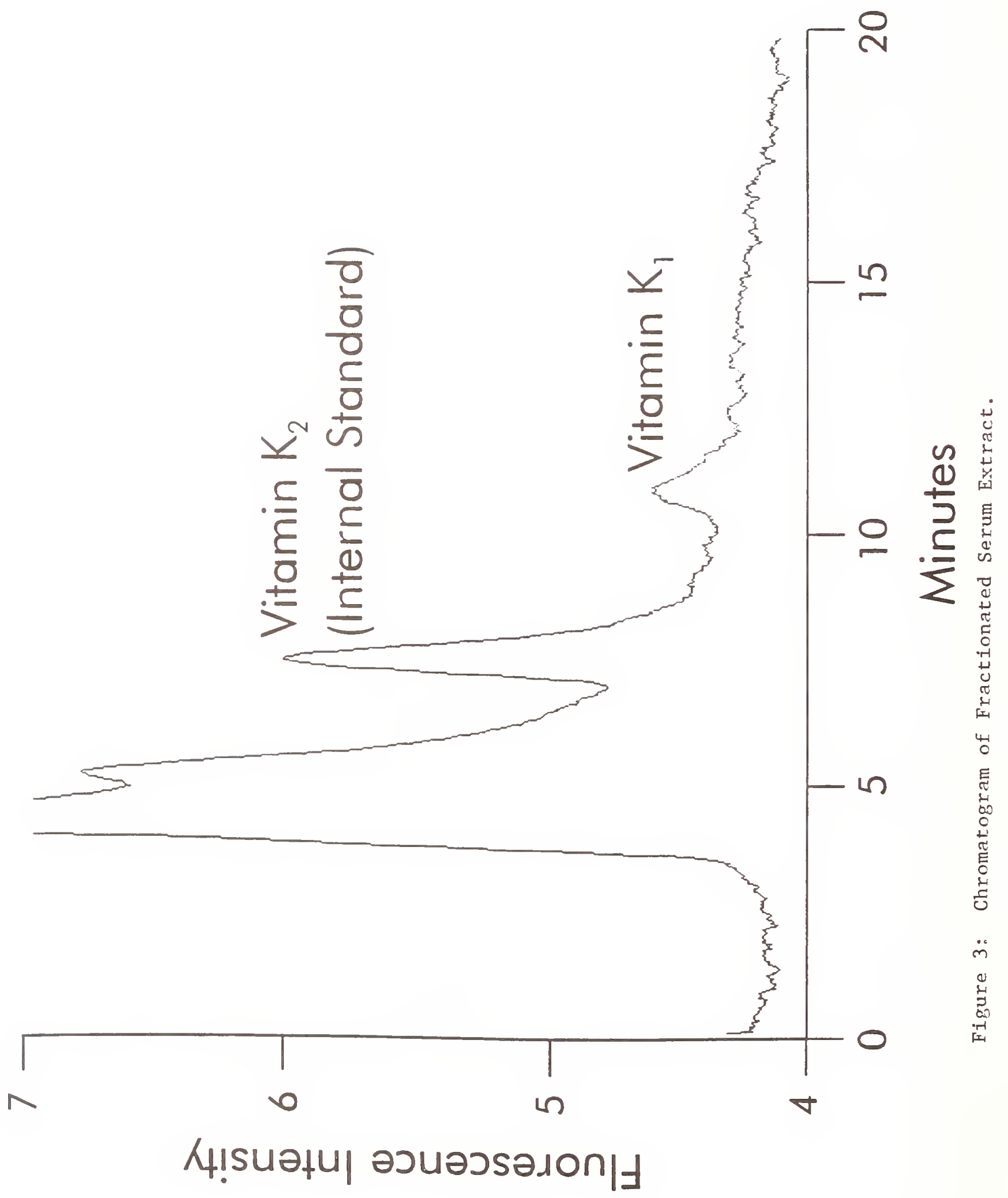




\section{MEASUREMENT OF (4-HYDROXYPHENYL)RETINAMIDE IN SERUM}

This section describes a method used by NIST for the determination of a retinoic acid derivative, (4-hydroxyphenyl)retinamide (4-HPR), in human serum. This compound is currently being evaluated for its cancer preventive effects in Phase II clinical trials sponsored by the NCI. Listed below are important factors related to this analysis.

\section{Important Factors}

\section{Calibration and Sample Preparation}

- Add the internal standard (4-ethoxyphenyl)retinamide (4-EPR) to the protein precipitation solvent.

- The liquid-liquid extraction protocol outlined in Clinical Chemistry 33, 1585-1592 (1987) should be used. This paper can be found beginning with page 1-28.

- Sera should be shipped on dry ice and frozen for storage.

- Care should be taken to prevent photodecomposition of the retinamides.

- The extraction volume correction is based on the recovery of the internal standard.

- Use a multiple-point calibration curve.

\section{Chromatography}

- The recommended column is a polymerically bonded $5 \mu \mathrm{m} \mathrm{C}_{18}$ column $(0.46 \mathrm{~cm} \times 25 \mathrm{~cm})$.

- Use an isocratic mobile phase: 55\% acetonitrile, $10 \%$-butanol, 35\% water, $0.01 \mathrm{~mol} / \mathrm{L}$ ammonium acetate.

- Variable wavelength detection should be used: programmed single wavelength at $364 \mathrm{~nm}$ for the first 12 minutes, $325 \mathrm{~nm}$ for the next 3 minutes, and $364 \mathrm{~nm}$ for the last 8 minutes (use wavelength timing to correspond to the elution times of 4-HPR, retinol, and (4-methoxyphenyl)retinamide and 4-EPR, respectively).

For further information, please contact William MacCrehan at (301)975-3122. 
NIST Measurements of (4-Hydroxyphenyl)retinamide in Serum

\author{
William A. MacCrehan
}

NIST developed a method for the determination of (4-hydroxyphenyl)retinamide (4-HPR, an amide of retinoic acid, see Figure 1), in 1987/88 as part of the NCI/NIST Micronutrients Measurement Quality Assurance Program. As a candidate cancer chemopreventive agent, accurate serum measurements are important to determine 4-HPR dosage compliance and pharmacokinetic behavior in phase 1 and 2 human clinical trials. The NIST method simultaneously determines the parent compound, the major metabolite (4methoxyphenyl)retinamide (4-MPR), and serum retinol. A non-naturally occurring compound, (4-ethoxyphenyl)retinamide (4-EPR) is used in the procedure as an internal standard.

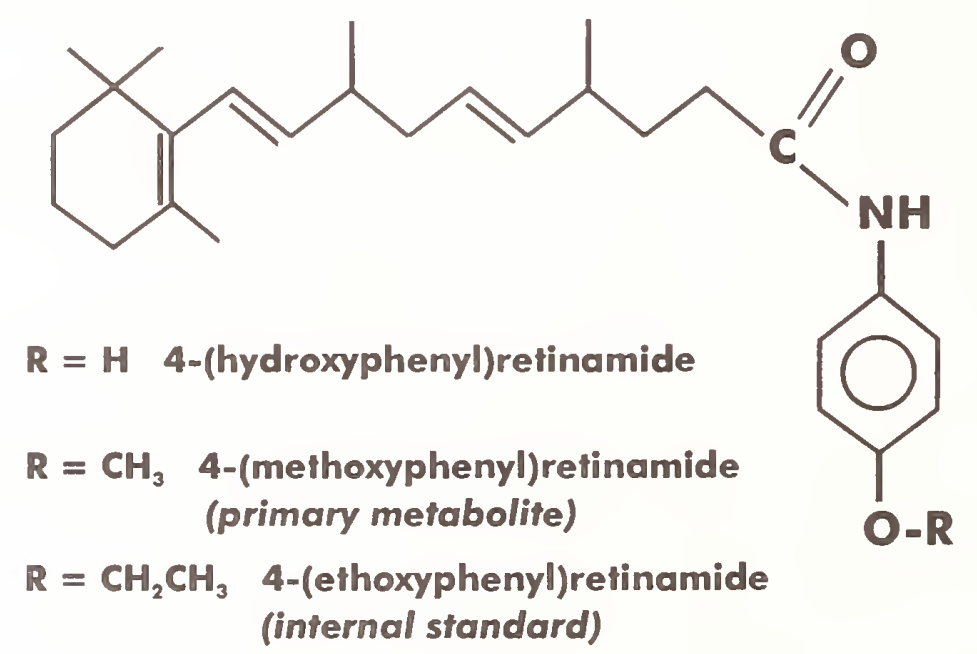

Figure 1: Structure of Retinamides.

The serum preparation procedure follows the liquid-liquid extraction protocol outlined in Clinical Chemistry 33, 1585-1592 (1987). Sera were shipped frozen on dry ice and stored at $-70{ }^{\circ} \mathrm{C}$ prior to analysis, and thawed thoroughly to room temperature before subsampling. Extra care was taken to avoid exposure to light as the retinamides are particularly sensitive to photodecomposition (as communicated by McNeil Pharmaceuticals) and the crystalline standards were stored at $-4{ }^{\circ} \mathrm{C}$. Calibration solutions were value-assigned based on gravimetric measurement of the pure crystalline compounds provided by $\mathrm{McNeil}$, and corrected for impurities separated by reversed-phase liquid chromatography with absorbance detection at $364 \mathrm{~nm}$ by dividing the peak area of the target analyte by the total area. Calibration was done using a mixture of the three retinamides in ethanol at several levels in the 0.1 to $0.5 \mu \mathrm{g} / \mathrm{mL}$ range. The method detection limit was approximately $0.005 \mu \mathrm{g} / \mathrm{mL}$ on 
a serum basis. The chromatographic separation conditions for the determination were as follows:

* column: Vydac 201TP $(5 \mu \mathrm{m}) 0.46 \times 25 \mathrm{~cm}$

* isocratic mobile phase: $55 \%$ acetonitrile, $10 \%$-butanol, $35 \%$ water, $0.01 \mathrm{~mol} / \mathrm{L}$ ammonium acetate

* detector: programmed single wavelength at $364 \mathrm{~nm}$ for the first 12 minutes, $325 \mathrm{~nm}$ for the next 3 minutes, and $364 \mathrm{~nm}$ for the last 8 minutes (wavelength timing to correspond to the elution times of 4-HPR, retinol, and 4-MPR and 4-EPR, respectively)

* sample size: $10 \mu \mathrm{L}$

On a set of test sera, we found that 4-HPR values ranged from 0.02 to 0.37 , retinol from 0.28 to 0.63 and 4-MPR from less than 0.005 to $0.31 \mu \mathrm{g} / \mathrm{mL}$. 


\section{NTST Technical Publications}

\section{Periodical}

Journal of Research of the National Institute of Standards and Technology-Reports NIST research and development in those disciplines of the physical and engineering sciences in which the Institute is active. These include physics, chemistry, engineering, mathematics, and computer sciences. Papers cover a broad range of subjects, with major emphasis on measurement methodology and the basic technology underlying standardization. Also included from time to time are survey articles on topics closely related to the Institute's technical and scientific programs. Issued six times a year.

\section{Nonperiodicals}

Monographs-Major contributions to the technical literature on various subjects related to the Institute's scientific and technical activities.

Handbooks-Recommended codes of engineering and industrial practice (including safety codes) developed in cooperation with interested industries, professional organizations, and regulatory bodies.

Special Publications-Include proceedings of conferences sponsored by NIST, NIST annual reports, and other special publications appropriate to this grouping such as wall charts, pocket cards, and bibliographies.

National Standard Reference Data Series-Provides quantitative data on the physical and chemical properties of materials, compiled from the world's literature and critically evaluated. Developed under a worldwide program coordinated by NIST under the authority of the National Standard Data Act (Public Law 90-396). NOTE: The Journal of Physical and Chemical Reference Data (JPCRD) is published bimonthly for NIST by the American Chemical Society (ACS) and the American Institute of Physics (AIP). Subscriptions, reprints, and supplements are available from ACS, 1155 Sixteenth St., NW, Washington, DC 20056.

Building Science Series-Disseminates technical information developed at the Institute on building materials, components, systems, and whole structures. The series presents research results, test methods, and performance criteria related to the structural and environmental functions and the durability and safety characteristics of building elements and systems.

Technical Notes--Studies or reports which are complete in themselves but restrictive in their treatment of a subject. Analogous to monographs but not so comprehensive in scope or definitive in treatment of the subject area. Often serve as a vehicle for final reports of work performed at NIST under the sponsorship of other government agencies.

Voluntary Product Standards-Developed under procedures published by the Department of Commerce in Part 10, Title 15, of the Code of Federal Regulations. The standards establish nationally recognized requirements for products, and provide all concerned interests with a basis for common understanding of the characteristics of the products. NIST administers this program in support of the efforts of private-sector standardizing organizations.

Order the following NIST publications_FIPS and NISTIRs—from the National Technical Information Service, Springfield, VA 22161.

Federal Information Processing Standards Publications (FIPS PUB)-Publications in this series collectively constitute the Federal Information Processing Standards Register. The Register serves as the official source of information in the Federal Government regarding standards issued by NIST pursuant to the Federal Property and Administrative Services Act of 1949 as amended, Public Law 89-306 (79 Stat. 1127), and as implemented by Executive Order 11717 (38 FR 12315, dated May 11, 1973) and Part 6 of Title 15 CFR (Code of Federal Regulations).

NIST Interagency Reports (NISTIR)-A special series of interim or final reports on work performed by NIST for outside sponsors (both government and nongovernment). In general, initial distribution is handled by the sponsor; public distribution is by the National Technical Information Service, Springfield, VA 22161, in paper copy or microfiche form. 
U.S. Department of Commerce

National Institute of Standards and Technology

Gaithersburg, MD 20899-0001

Official Business

Penalty for Private Use $\$ 300$ 